\title{
Dynamics of Hydrogen Atoms Scattering from Surfaces
}

\author{
Dissertation \\ zur Erlangung des mathematisch-naturwissenschaftlichen Doktorgrades \\ Doctor of Philosophy \\ der Georg-August-Universität Göttingen
}

im Promotionsprogramm Chemie

der Georg-August University School of Science (GAUSS)

vorgelegt von
Hongyan Jiang

aus Harbin, China

Göttingen, 2016.12.09. 


\section{$\underline{\text { Betreuungsausschuss }}$}

Prof. Alec M. Wodtke, Dynamics at Surfaces, Institute for Physical Chemistry/ Max-Planck-Institute for Biophysical Chemistry

Prof. Dirk Schwarzer, Dynamics at Surfaces, Institute for Physical Chemistry/ Max-Planck-Institute for Biophysical Chemistry

\section{Mitglieder der Prüfungskommission}

Referent: Prof. Alec M. Wodtke, Dynamics at Surfaces, Institute for Physical Chemistry/ Max-Planck-Institute for Biophysical Chemistry

Korreferent: Prof. Dirk Schwarzer, Dynamics at Surfaces, Institute for Physical Chemistry/ Max-Planck-Institute for Biophysical Chemistry

Weitere Mitglieder der Prüfungskommission:

Prof. Jürgen Troe, Institute for Physical Chemistry

Prof. Jörg Schröder, Institute for Physical Chemistry

Prof. Ricado Mata, Institute for Physical Chemistry

PD Dr. Thomas Zeuch, Abteilung Suhm, Institute for Physical Chemistry 


\section{Abstract}

Hydrogen atoms interactions with single crystal surfaces are the simplest processes in surface science, which are of both practical and fundamental interest. Chemical reactions of hydrogen atoms with single crystal surfaces lead to adsorption. Despite the fact that it has been studied for decades, the dynamics of hydrogen atom adsorption is still not fully understood. Adsorption involves the impinging hydrogen atom losing its initial translational energy and dissipating the energy of the chemical bond formed with the surface. How hydrogen atoms dissipate their initial translational energy to adsorb on a surface is still an open question. In principle, a hydrogen atom may lose its initial translational energy to the vibrations of the surface atoms or to electron-hole pair excitations. To answer this question in detail, a state-of-the-art UHV machine was built to study atom-surface scattering. During my doctoral study, I conducted experiments on hydrogen atom scattering from single crystal surfaces, including $\mathrm{Au}(111)$ and $\mathrm{Pt}(111), \mathrm{Xe}$ covered $\mathrm{Au}(111)$ and epitaxial graphene on $\operatorname{Pt}(111)$. High resolution scattering angle resolved translational energy loss distributions have been obtained. The goal of this research is to achieve a detailed understanding of the mechanisms of $\mathrm{H}$ atom interaction with different kinds of single crystal surfaces, and especially of the translational energy transfer between the atom and the surfaces, which is fundamentally important to the adsorption processes.

To compare hydrogen atom translational energy loss to metals and insulators, I studied scatterings of hydrogen atoms from $\mathrm{Au}(111)$ and Xe layer on $\mathrm{Au}(111)$. Hydrogen atoms scattering from insulating Xe layer exhibit a small energy loss and a narrow translational energy distribution and can be understood using a binary collision model. In contrast, Hydrogen atoms scattering from $\mathrm{Au}(111)$ show a large energy loss and a broad translational energy distribution, indicating that a broad continuum of accepter states in the solid contribute to the translational energy loss. A MD simulation self-consistently including the non-adiabatic electronic excitations agrees with the experiments of hydrogen atom scattering on metal. In contrast, calculations neglecting the electronic excitations cannot capture the essence of the measurements, indicating hydrogen atoms scattering from metal is strongly non-adiabatic. Exchanging $\mathrm{H}$ atoms with $\mathrm{D}$ atoms only leads to minor change in the translational energy loss distribution. This is explained 
by a cancellation effect, where the phonon excitation is enhanced for D but the electron-hole pair excitation is reduced.

To study the dynamics of chemically activated adsorption of hydrogen atoms, I did a series of experiments on hydrogen atoms scattering from epitaxial graphene, which has a barrier to $\mathrm{C}-\mathrm{H}$ bond formation of several hundreds of meV. The scattered hydrogen atoms exhibit a bimodal distribution for translational energy and scattering angle. The fast component in the distribution originates from atoms scattered back without crossing the adsorption barrier and is near-elastic and near-specular. The slow component originates from atoms scattered back after crossing the adsorption barrier and exhibits large and rapid energy loss (energy loss power around $10^{19}$ $\mathrm{W} / \mathrm{mole}$ atom). By monitoring the ratio between the slow and the fast component, we determine that the adsorption threshold is in the range of $0.41 \mathrm{eV}$ to $0.48 \mathrm{eV}$ for the $\mathrm{H}$ atom, and $0.43 \mathrm{eV}$ to $0.47 \mathrm{eV}$ for the D atom. Combined with DFT based AIMD calculations, we conclude that the fast component is H/D atom quasi-elastic scattering on a corrugated surface. The large and rapid energy loss of the slow component is caused by the formation of a transient reaction complex. Due to the $\pi$ resonance structure of graphene, $\mathrm{H}$ atom colliding with one $\mathrm{C}$ atom will cause simultaneous displacements of the neighboring $\mathrm{C}$ atoms, leading to the formation of a transient reaction complex. Large portion of the energy loss is due to the inelastic scattering mechanism. Surface IVR provides insights of the phonon excitations in graphene. 


\section{Contents}

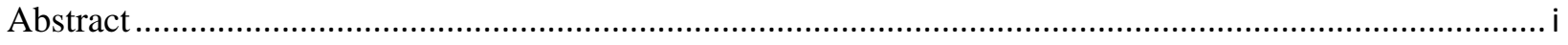

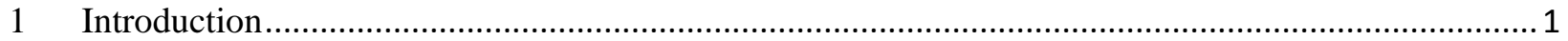

2 Previous Studies on Atom-Surface Interaction: Theories and Experiments ...................................... 3

2.1 Baule model and noble gas atoms scattering from surfaces ........................................................ 3

2.2 Potential energy surface (PES) and molecular dynamics (MD) .................................................. 6

2.2.1 Empirical potential energy curve and atom diffraction on surfaces........................................ 7

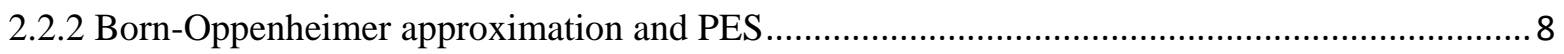

2.2.3 Molecular dynamics (MD) and ab initio molecular dynamics (AIMD) .................................. 10

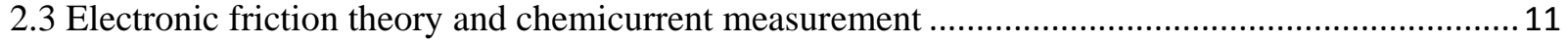

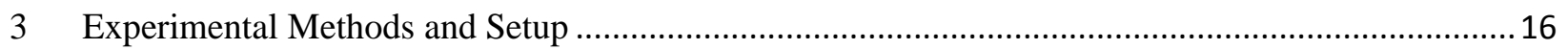

3.1 Mono-energetic narrow energy distribution H/D atom beams.................................................. 16

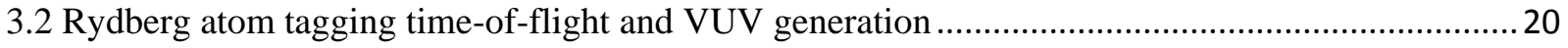

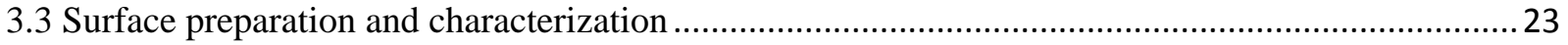

3.4 Laser system, experimental geometry and timing .................................................................... 27

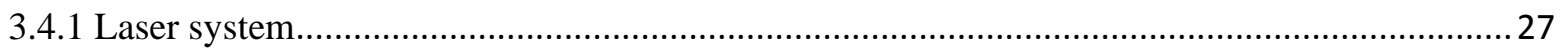

3.4.2 Experimental geometry and timing, and data acquisition ....................................................28

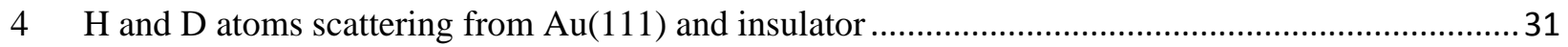

$4.1 \mathrm{H}$ atoms scattering from $\mathrm{Au}(111)$ and insulating Xe layer ....................................................... 31

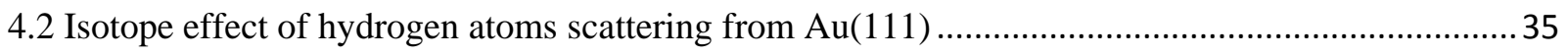

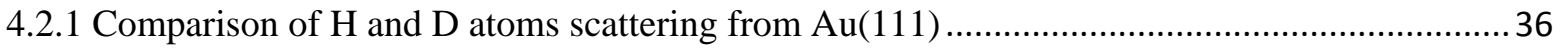

4.2.2 Discussion on the isotope effect of $\mathrm{H} / \mathrm{D}$ atoms scattering from $\mathrm{Au}(111)$..................................38

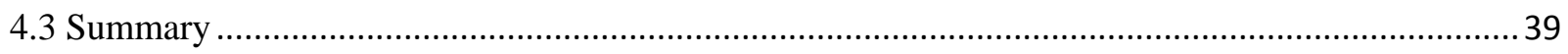

$5 \quad$ Hydrogen Atoms Interaction with Graphene: Background …...................................................... 40

5.1 Introduction to graphene properties related to atom scattering .....................................................40

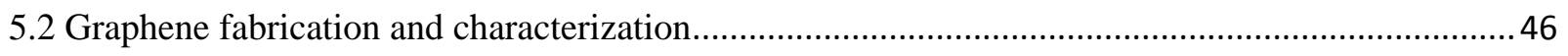

5.2.1 Brief introduction on graphene fabrication methods ....................................................... 46

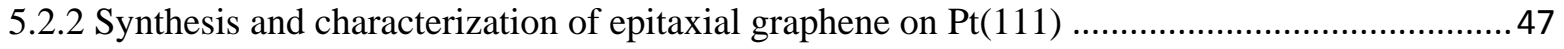

5.3 Graphene interaction with hydrogen atoms: Previous theories and experiments ...........................55

5.3.1 Energetic and structural studies of $\mathrm{H} / \mathrm{D}$ atoms adsorption on graphene ..................................5 55

5.3.2 Studies of dynamics of hydrogen atoms adsorption on graphene ............................................ 58 
6 Hydrogen Atoms scattering from Epitaxial Graphene on Pt(111): Adsorption Threshold and Energy Transfer.

6.1 Comparison between hydrogen atoms scattering from epitaxial graphene on $\operatorname{Pt}(111)$ and pristine $\operatorname{Pt}(111)$

$6.2 \mathrm{H} / \mathrm{D}$ atoms scattering from epitaxial graphene on $\mathrm{Pt}(111)$ under various incidence conditions ....... 64

6.2.1 $\mathrm{H}$ atoms scattering from epitaxial graphene as a function of incidence energy ........................66

6.2.2 $\mathrm{H}$ atoms scattering from epitaxial graphene as a function of incidence angle .........................67

6.2.3 $\mathrm{H}$ atoms scattering from epitaxial graphene as a function of surface temperature ...................71

6.2.4 $\mathrm{D}$ atoms scattering on epitaxial graphene on various incidence conditions .............................72

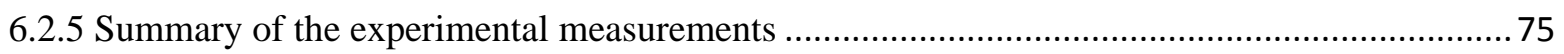

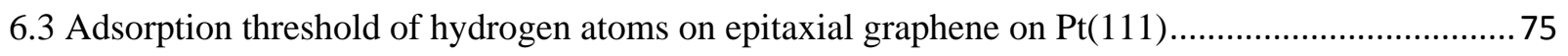

6.3.1 Determining adsorption thresholds of H/D atoms on epitaxial graphene …............................75

6.3.2 A model for fitting the adsorption threshold ....................................................................... 77

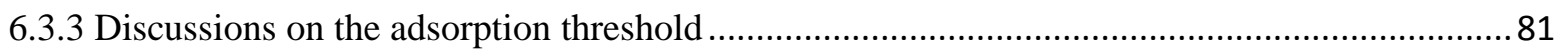

6.4 Dynamics of hydrogen atoms scattering from epitaxial graphene on $\operatorname{Pt}(111)$ : Two different

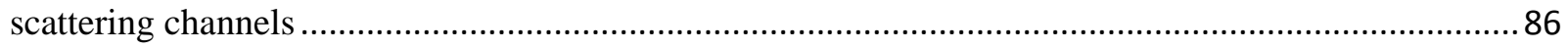

6.4.1 An overall comparison between the theoretical and experimental data ................................. 86

6.4.2 The fast component: Atoms scattering on a corrugated surface ..........................................90

6.4.3 The slow component: Transient reaction complex formation, tug-of-war inelastic scattering,

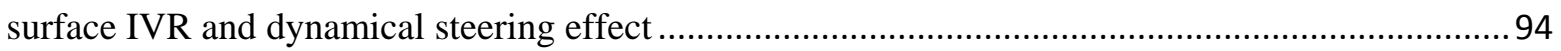

6.5 Summary on H/D atoms scattering from epitaxial graphene on $\operatorname{Pt}(111)$...................................110

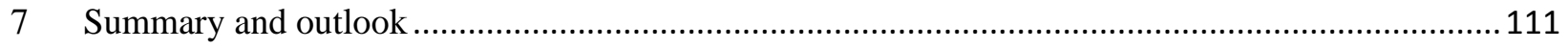

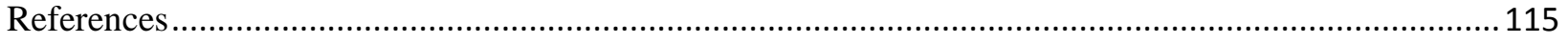

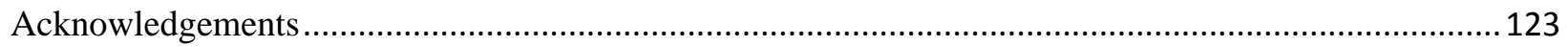




\section{Introduction}

Hydrogen is the simplest and most abundant element in the universe. It constitutes $75 \%$ of the baryonic mass of the universe. The fundamental nature (i.e. its simplicity) of hydrogen has led naturally to its preeminent role in the history of science. Attempts to develop a theoretical understanding of the hydrogen atom opened the gate to quantum theory. The 1927 article of Walter Heitler and Fritz London on $\mathrm{H}_{2}$ is the first calculation of chemical bonding based on quantum mechanical considerations (1). Using the simplest chemical reaction $\mathrm{H}+\mathrm{H}_{2} \rightarrow \mathrm{H}_{2}+\mathrm{H}$ as a model, Eyring and Polanyi for the first time formulated an electronically adiabatic potential energy surface (PES) in 1931 (2). After this first study on hydrogen-exchange reaction, a tremendous amount of research was done on the simple $\mathrm{H}+\mathrm{H}_{2}$ system (3-5). Detailed studies on this system have greatly advanced and are still improving our understanding of chemical reaction mechanisms $(6,7)$.

Similar to its gas phase relative $\mathrm{H}+\mathrm{H}_{2}$ reaction, hydrogen atom interactions with single crystal surfaces are the simplest processes in surface science. Chemical reactions of hydrogen atoms with single crystal surfaces lead to adsorption, which is of both practical and fundamental interest. Hydrogen atom adsorption plays an important role in many technologies, for instance nuclear fusion in tokamaks (8), hydrogen storage (9), industrial surface processing (10) and heterogeneous catalysis (11). Owing to its simplicity compared to other systems, it is also favored in theoretical simulations (12). Adsorption involves the impinging hydrogen atom losing its initial translational energy and dissipating the energy of the chemical bond formed with the surface. If it is a chemical activated process, it also includes adsorption barrier crossing. In principle, a hydrogen atom may lose its initial translational energy in two ways after crossing an adsorption barrier. The first way involves transfer of translational energy to the vibrations of the surface atoms (phonons). Translational energy loss to insulator surfaces mainly occurs by this mechanism. On the other hand, for metal and semiconductor surfaces, where low-lying electronhole pairs (ehps) are available, excitation of ehps may also occur. For years, chemists have been trying to understand the question of to what extent the translational energy loss proceeds adiabatically (via phonon) or non-adiabatically (via ehps excitations). This is related to a broader question of whether the scattering of molecules and atoms can be accurately described within the 
so called Born-Oppenheimer approximation (13), which is the basis of the "standard model of chemical reactivity" $(14,15)$.

Nienhaus et al. experimentally confirmed that hydrogen atom adsorption on $\mathrm{Cu}$ and $\mathrm{Ag}$ indeed creates ehps (16-21), but little is known about whether hydrogen atom translational energy loss to metal and semiconductor surfaces is dominated by adiabatic phonon excitations or nonadiabatic ehps excitations within chemically interesting energy ranges. Some theoretical work suggests that the coupling between translational energy and ehps makes minor contribution (22), while other work implies that it is comparable to the coupling to phonons or the dominant process in energy loss (23-25). Until now, no experimental results exist on this question.

Surface scattering under UHV conditions has shown its capability in reaction dynamics studies, especially those on non-adiabatic ehps excitations on metal surfaces. With tunable narrow bandwidth lasers, state-to-state scattering cross sections have been resolved. Surface scattering experiments have demonstrated that vibrational energy transfer between molecules and metal surfaces are strongly non-adiabatic (26). Although the first study of hydrogen atoms scattering from a single crystal surface was conducted decades ago, such systems have received very little experimental attention to date. In the early 1930's, Johnson demonstrated hydrogen atom diffraction on LiF crystal (27). More than 40 years later, Finzel et al. performed hydrogen atom diffraction on alkali halide surfaces again (28). The lack of hydrogen atom-surface scattering experiments is mainly due to experimental difficulties involved in producing narrow energy distribution $\mathrm{H}$ atom beams with tunable energy and detecting them with high resolution and efficiency.

In this thesis, I present the most recent results on $\mathrm{H}$ atom scattering from single crystal surfaces, including $\mathrm{Au}(111)$ and $\mathrm{Pt}(111)$, Xe covered $\mathrm{Au}(111)$ and epitaxial graphene on $\mathrm{Pt}(111)$. On a recently built machine, we generate monoenergetic atomic hydrogen beams with tunable energy and detect them with high efficiency. Employing the Rydberg atom tagging method, high resolution scattering angle resolved time-of-flight (ToF) distributions have been measured (29). The goal of this research is to achieve a detailed understanding of the mechanisms of $\mathrm{H}$ atom interaction with different kinds of single crystal surfaces, and especially of the translational energy transfer between the atom and the surfaces, which is fundamentally important to adsorption processes. 


\section{Previous Studies on Atom-Surface Interaction: Theories and Experiments}

Establishing theories that can explain and predict experimental findings is a key endeavor in physical chemistry. In this chapter, existing theories or models that are commonly used to describe atom surface interactions are reviewed. Representative experiments that can be interpreted by these theories will also be included.

\subsection{Baule model and noble gas atoms scattering from surfaces}

One of the simplest microscopic models to describe atom-surface interaction is the Baule binary collision model. The model assumes the interaction as a hard sphere collision between an incoming atom and a surface atom. The collision obeys momentum and energy conservation.

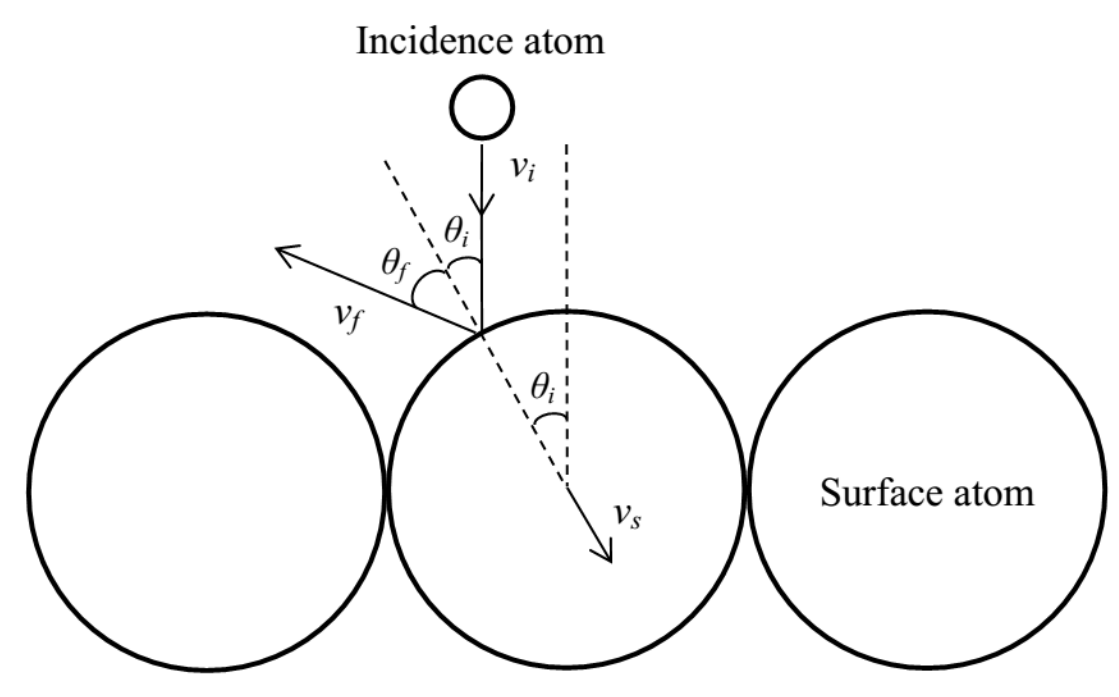

Figure 2.1: Binary collision between 2 atoms.

As is shown in Figure 2.1, an incidence atom with a velocity $v_{i}$ collides with a surface atom initially at rest. Consider the case of an incoming atom that hits the surface atom with an angle of $\theta_{i}$ with respect to the normal at the collision point, and rebounds with a velocity of $v_{f}$ at an angle of $\theta_{f}$ relative to the normal. For hard sphere collisions, all the forces of the collision are transmitted along the normal direction at the collision point. So the momentum component of the 
scattered atom perpendicular to the normal at the collision point remains unchanged. According to momentum and energy conservation, we obtain:

$$
\begin{gathered}
m_{a} v_{i} \sin \theta_{i}=m_{a} v_{f} \sin \theta_{f} \\
m_{a} v_{i} \cos \theta_{i}=m_{a} v_{f} \cos \theta_{f}+m_{s} v_{s} \\
\frac{1}{2} m_{a} v_{i}^{2}=\frac{1}{2} m_{a} v_{f}^{2}+\frac{1}{2} m_{s} v_{s}^{2}
\end{gathered}
$$

where $m_{a}$ and $m_{s}$ are the masses of the incoming and surface atom respectively, and $v_{s}$ is the velocity of the surface atom after the collision. From equations 2.1-2.3, we obtain the fraction of translational energy retained by the scattered atom:

$$
B=\frac{E_{f}}{E_{i}}=\frac{v_{f}^{2}}{v_{i}^{2}}=1-\frac{4 m_{a} m_{s} \cos ^{2} \theta_{i}}{\left(m_{a}+m_{s}\right)^{2}}
$$

The maximum energy loss for the hard-sphere binary collision is obtained, when the angle $\theta_{i}$ equals zero, and $B=\frac{\left(m_{a}-m_{s}\right)^{2}}{\left(m_{a}+m_{s}\right)^{2}}$ is called the Baule limit. The simple scenario above implicitly assumes that the incoming atom only collides with one surface atom and that all the surface atoms are disconnected from one another. It is called the soft surface limit. Most of the times, this is not the case. The surface is stiffer due to the binding of surface atoms. Instead of colliding with one atom with a mass $m_{s}$, the incoming atom will see several surface atoms with an effective mass $m_{s}^{*}$. This requires changing the mass from $m_{s}$ to $m_{s}^{*}$ in the equations above.

Other more sophisticated models have been developed, which are based on the Baule model but include effects of attractive potentials (attractive Baule), surface temperature and surface roughness. However, the common essence of these models is to consider the collision between the surface and the incoming atoms as elastic sphere collisions. Although it contains some coarse assumptions, the Baule model provides a qualitatively - sometimes even quantitatively — good description of energy transfer for atom-surface scattering processes, especially for non-reactive 
atom-surface scattering. In the 1980s, D. J. Auerbach et al. did a series of experiments on noble gases scattering from single crystal surfaces (30), for which the Baule model provides qualitatively good agreement with the experimental results. In this section, Ar atom scattering from $\operatorname{Pt}(111)$ is presented as an example $(31,32)$. The experimental setup is depicted in Figure 2.2 .

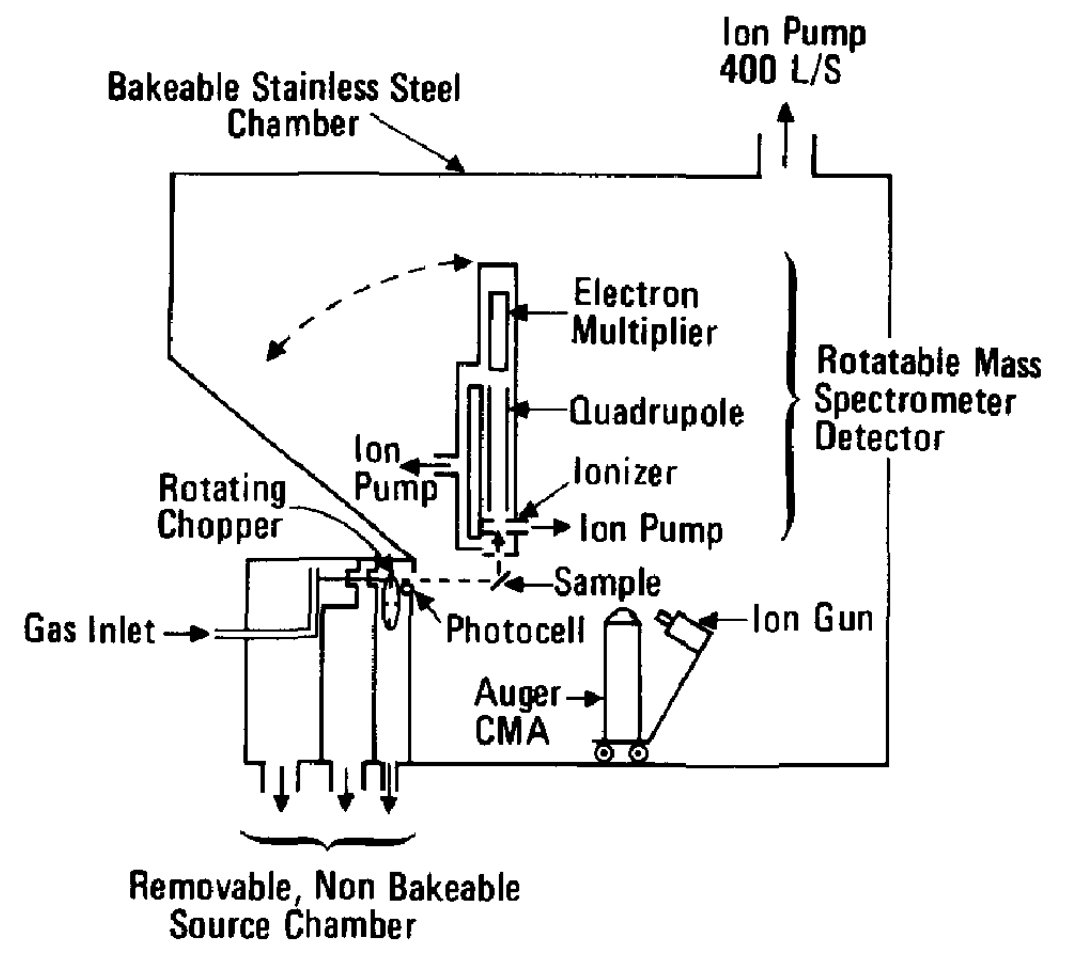

Figure 2.2: Experimental setup of Ar atom scattering from Pt(111). Reprinted from Ref. (32), with the permission of AIP Publishing.

The Ar atom beam was generated in the source chamber by expansion of Ar gas from a high pressure region (typically 2-3 bar) through a small orifice (typically several tens $\mu \mathrm{m}$ in diameter) into a low pressure region (typically $10^{-2}$ Torr). The resulting supersonic beam was skimmed, collimated, and chopped into pulses of $20 \mu \mathrm{FWHM}$ at a frequency of several hundreds of $\mathrm{Hz}$. The kinetic energy of the atomic beam was controlled by varying the temperature of the nozzle or seeding the Ar gas in lighter or heavier gases. The single crystal surface sample was held on a sample mount in the UHV chamber. The scattered atoms were detected by a rotatable, differentially pumped quadrupole mass spectrometer with an angular resolution of $1^{\circ}$. The mechanical chopper provided the time zero reference, allowing a time-of-flight distribution to be 
measured. The scattered atoms exhibited a narrow angular distribution, indicating direct scattering. Figure 2.3 shows the time-of-flight distribution of Ar atom scattering from $\operatorname{Pt}(111)$ at a $45^{\circ}$ incidence angle. The energy of the scattered Ar atoms decreases as the scattering angle increases. This can be understood as the conservation of the parallel component of momentum and kinetic energy of the Ar atom. If the Ar atom is scattered by only one Pt atom, the energy distribution should peak around $E_{\text {out }}=0.71 E_{i}$ at $45^{\circ}$ scattering angle. The experimental results show $E_{\text {out }}=0.84 E_{i}$ at $45^{\circ}$ scattering angle. This gives an effective mass for the Ar atom scattering from $\mathrm{Pt}(111)$ around $2.5 m_{P t}$.

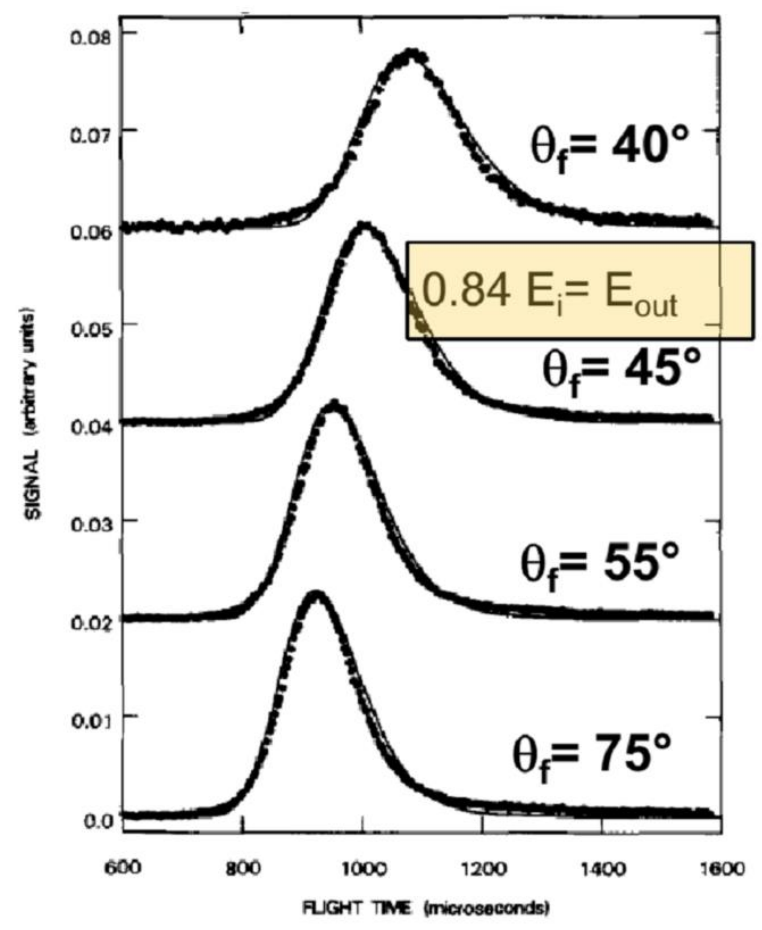

Figure 2.3: Time-of-flight spectrum of Ar atom scattering from Pt(111). Incidence angle is $45^{\circ}$, and $\theta_{f}$ indicates scattering angle. Reprinted from Ref. (32), with the permission of AIP Publishing.

\subsection{Potential energy surface (PES) and molecular dynamics (MD)}

The Baule model assumes that the atom-surface interaction is an instantaneous collision. In a more realistic model, the incoming atom would experience forces in the vicinity of the surface. Atoms could experience a physisorption potential energy well $\left(E_{\mathrm{phy}}\right)$, or overcome a barrier $\left(E_{\mathrm{b}}\right)$ and meet a chemisorption well $\left(E_{\text {chem }}\right)$ on the surfaces. Forces experienced by the incidence 
particles could be van der Waals interaction or chemical bonding with surface atoms. In this chapter, I will present some methods for describing the forces between atoms and surfaces.

\subsubsection{Empirical potential energy curve and atom diffraction on surfaces}

Atoms and molecules are described on a microscopic level by quantum theories, but quantum theories are usually difficult to implement due to their complexity. Hence, empirical potential energy models are still useful and even necessary in many cases. Frequently used is the LennardJones potential, which describes van der Waals interaction. For a pairwise interaction it is expressed as:

$$
V(z)=\varepsilon\left[\left(\frac{\sigma}{z}\right)^{12}-2\left(\frac{\sigma}{z}\right)^{6}\right]
$$

where $z$ is the distance between two interacting particles, $\varepsilon$ is the minimum of the potential well, and $\sigma$ is the equilibrium distance between the two particles. The negative term represents the van der Waals attraction. Pauli repulsion is represented by the positive term, which can be replaced by other functions, for example Morse-like exponential function.

The Lennard-Jones potential and its revised versions are good at describing physisorption. This can be seen in a series of atom diffraction experiments, in which low energy atom diffraction resonances were used to characterize the physisorption potential. Ghio et al. proposed a pairwise integrated potential to describe the interaction of nonpolar molecules with single-layer graphite (33).

$$
V^{*}(z)=\gamma_{1} * \varepsilon^{*}\left[\frac{1}{\gamma_{1}}\left(\frac{\sigma^{*}}{z}\right)^{10}-\gamma_{2}\left(\frac{\sigma^{*}}{z}\right)^{6}-\left(\frac{\sigma^{*}}{z}\right)^{4}\right]
$$

where $\varepsilon^{*}$ is the physisorption well minimum, $\sigma^{*}$ is the equilibrium distance, and $\gamma_{1}$ and $\gamma_{2}$ are weighting factors. The positive $z^{-10}$ term represents the Pauli repulsion, and the $z^{-4}$ and $z^{-6}$ terms represent different order van der Waals interactions. Low-energy narrow speed distribution $\mathrm{H}$ atom beams generated by radio frequency discharge of $\mathrm{H}_{2}$ molecules are diffracted by a graphite sample. The interaction potential shown in Eq. 2.6 is then used to fit the measured diffraction resonance energies. This provided a $40 \mathrm{meV}$ physisorption well for $\mathrm{H}$ atoms on graphene (33). 


\subsubsection{Born-Oppenheimer approximation and PES}

The dynamics of atoms and molecules are described by quantum mechanics. In the energy range of chemical interest, the system can be described by the non-relativistic Schrödinger equation. The non-relativistic Hamiltonian for a system of interacting atoms is

$$
H=-\sum_{i=1}^{N} \frac{\hbar^{2}}{2 M_{i}} \nabla_{\boldsymbol{R}_{i}}^{2}-\sum_{i=1}^{n} \frac{\hbar^{2}}{2 m_{e}} \nabla_{\boldsymbol{r}_{i}}^{2}-\sum_{i, j}^{N, n} \frac{Z_{i} e^{2}}{\left|\boldsymbol{R}_{i}-\boldsymbol{r}_{j}\right|}+\sum_{i, j, i>j}^{N} \frac{Z_{i} Z_{j} e^{2}}{\left|\boldsymbol{R}_{i}-\boldsymbol{R}_{j}\right|}+\sum_{i, j, i>j}^{n} \frac{e^{2}}{\left|\boldsymbol{r}_{i}-\boldsymbol{r}_{j}\right|},
$$

where $\boldsymbol{R}$ and $\boldsymbol{r}$ denote the positions of the nuclei and electrons, respectively. Solving the complete Schrödinger equation is a daunting task due to its complexity. However, when the motion of electrons and nucleus are separated, the equation becomes simpler to solve, and it can be applied to chemically relevant systems. The so-called Born-Oppenheimer approximation is based on the mass difference of electrons and nuclei. The mass of the proton is 1836 times greater than that of the electron. Due to this large mass difference, electrons respond approximately instantaneously to the much slower motion of the nuclei. Hence the total Hamiltonian can be separated into two parts:

$$
H=-\sum_{i=1}^{N} \frac{\hbar^{2}}{2 M_{i}} \nabla_{R_{i}}^{2}+H_{e l}(\boldsymbol{r} ; \boldsymbol{R})
$$

Here, $H_{e l}(\boldsymbol{r} ; \boldsymbol{R})$ is the entire Hamiltonian of the system with the exception of the kinetic energy operator for the slow nuclei, and can be viewed as the Hamiltonian that governs the electrons when the nuclei are fixed at positions $\boldsymbol{R}$. One now can define adiabatic electronic wave functions $\Phi_{n}(\boldsymbol{r} ; \boldsymbol{R})$ to be eigenfunctions of $H_{e l}(\boldsymbol{r} ; \boldsymbol{R})$ for fixed $\boldsymbol{R}$.

$$
H_{e l}(\boldsymbol{r} ; \boldsymbol{R}) \Phi_{n}(\boldsymbol{r} ; \boldsymbol{R})=\mathcal{E}_{n}(\boldsymbol{R}) \Phi_{n}(\boldsymbol{r} ; \boldsymbol{R})
$$

Note that $\varepsilon_{n}(\boldsymbol{R})$ and $\Phi_{n}(\boldsymbol{r} ; \boldsymbol{R})$ depend parametrically on the nuclear positions $\boldsymbol{R}$. This is denoted by the semicolon in the expression. $\varepsilon_{n}(\boldsymbol{R})$ is called the adiabatic or Born-Oppenheimer potential energy surface (PES) corresponding to the electronic state $n$. The word "adiabatic" comes from the adiabatic theorem of Max Born and Vladimir Fock. It assumes that the electronic wave functions $\Phi_{n}(\boldsymbol{r} ; \boldsymbol{R})$ adjust themselves instantaneously as the nuclei positions $\boldsymbol{R}$ change, such that they are always eigenfunctions of $H_{e l}(\boldsymbol{r} ; \boldsymbol{R})$. Then the exact wave function $\Psi(\boldsymbol{r}, \boldsymbol{R})$ can be expressed as a linear combination of the $\Phi_{n}(\boldsymbol{r} ; \boldsymbol{R})$ basis functions. 


$$
\Psi(\boldsymbol{r}, \boldsymbol{R})=\sum_{n} \Phi_{n}(\boldsymbol{r} ; \boldsymbol{R}) \psi_{n}(\boldsymbol{R})
$$

Substituting wave function Eq. 2.10 and Hamiltonian Eq. 2.8 into the Schrödinger equation, multiplying from the left by $\Phi_{m}^{*}(\boldsymbol{r} ; \boldsymbol{R})$, integrating over all electronic coordinates $\boldsymbol{r}$, and neglecting the first and second derivative of $\Phi_{n}(\boldsymbol{r} ; \boldsymbol{R})$ with respect to nuclear coordinates $\boldsymbol{R}$, we obtain a set of Schrödinger equations describing the nuclear motions:

$$
\left[-\sum_{i=1}^{N} \frac{\hbar^{2}}{2 M_{i}} \nabla_{R_{i}}^{2}+\varepsilon_{n}(\boldsymbol{R})\right] \psi_{n}(\boldsymbol{R})=E \psi_{n}(\boldsymbol{R}),
$$

where $E$ is the total energy of the system, and $\psi_{n}(\boldsymbol{R})$ is the nuclear wave function. The nuclear motion is governed by Eq. 2.11, with the potential given by $\varepsilon_{n}(\boldsymbol{R})$. The $\varepsilon_{n}(\boldsymbol{R})$ is in turn obtained from Eq. 2.9 for each nuclear geometry, $\boldsymbol{R}$. In this way, we separate the nuclear motion from the electronic motion.

The Born-Oppenheimer approximation allows the PES to be calculated from ab initio theories (quantum mechanical theories). The PES provides a picture of chemical interaction without considering the electrons explicitly. Once the PES has been determined, explicit knowledge of electronic motion is not required, and the chemical dynamics can be described in terms of nuclei moving on a single electronic potential energy surface obtained from Eq. 2.9.

Constructing a PES usually involves electronic energy calculation at different nuclear configurations, and subsequent fitting of calculated values to an analytical form. Methods that are used to calculate electronic structures can be classified into two main branches: wave function methods and density functional theory (DFT) $(34,35)$. As the name implies, wave function methods are based on construction of many-body wave functions. The Hartree-Fock method (HF), also known as the self-consistent field method (SCF), is the most basic wavefunction method. Other wave-function methods-including Møller-Plesset (MP) (36), configuration interaction (CI) and coupled cluster (CC) (37) - provide corrections for electron correlations that are neglected in the mean-field approximation that is employed in the HF calculation. When large basis sets are employed, wave function methods can provide quite accurate results. CC calculations including double and triple excitations are known as the "gold standard" methods in quantum chemistry. Coupled cluster calculations have been used to 
reproduce the most state-of-the-art experimental measurements (38). However, high-level wave function methods are computationally expensive. Although a number of surface science studies have employed wave function methods (39), such methods are more suitable for systems consisting of only a few small atoms. Another choice for the electronic structure calculation is DFT $(40,41)$. With proper exchange-correlation functionals and periodic boundary conditions, DFT produces sufficiently accurate results for surface studies, while maintaining a relatively low computational cost.

\subsubsection{Molecular dynamics (MD) and ab initio molecular dynamics (AIMD)}

Once the PES is constructed, the atomic motion can be described fully quantum mechanically (within the BO limit) by solving the nuclear Schrödinger equation Eq. 2.11. The full quantum mechanical approach (QM) has succeeded in describing gas phase reactions of small molecules. As mentioned before, the computational cost is again the limiting factor for complete quantum dynamical calculations. The large number of nuclear degrees of freedom often prohibits the implementation of QM in surface dynamics simulations. There have been some QM studies on surface dynamics, which only consider a limited number of degrees of freedom. However, such approaches lead to uncertainty which arises from the incomplete description of the system (14).

Another approach, molecular dynamics simulation (MD), treats the atomic motions classically. The motion of an atom on a particular PES is obtained from Newton's equation:

$$
M_{i} \ddot{\boldsymbol{R}}_{i}=-\nabla_{\boldsymbol{R}_{i}} \mathcal{E}_{n}(\boldsymbol{R})
$$

With proper propagation algorithms, MD simulations produce quantitatively accurate results while keeping the computation time relatively short. This makes MD simulations popular in surface science studies.

The accuracy of MD simulations strongly depends on the quality of the PES, for which fitting errors are usually unavoidable. To overcome this problem, ab initio molecular dynamics (AIMD) can be used (42). Instead of generating a PES, AIMD calculates the forces on-the-fly. Electronic structures are calculated at every step of the simulation. An instantaneous force field is then generated and is used to propagate the MD simulation. AIMD is obviously more computationally 
expensive than MD, but it can be used to provide benchmark data for validating different potential models for MD simulations.

MD simulations also neglect quantum effects such as zero point energy, tunneling and quantum resonance. In many cases, these effects play an important role, especially for light species like the hydrogen atom. Improvements have been made by treating the system partially quantum mechanically. A second level Born-Oppenheimer separation of fast and slow nuclear motions can be used to account for this problem (14).

\subsection{Electronic friction theory and chemicurrent measurement}

The Born-Oppenheimer approximation is a fundamental assumption behind adiabatic theories such as molecular dynamics and $a b$ initio molecular dynamics. It is assumed that nuclei move on a single potential energy surface and that different electronic states are not coupled to one another by the nuclear motion. In order to obtain the nuclear Schrödinger equation Eq. 2.11, the terms containing the first and second derivative of electronic wave function $\Phi_{n}(\boldsymbol{r} ; \boldsymbol{R})$ with respect to the nuclear coordinates $\boldsymbol{R}$ have been neglected. Assuming a semi-classical description of the system, in which the nuclear motion can be represented by a classical trajectory $\boldsymbol{R}=\boldsymbol{R}(\boldsymbol{t})$, the adiabatic behavior can be assured by Massey's criterion:

$$
\hbar \dot{\boldsymbol{R}}_{i}\left|\frac{\left\langle\Phi_{m}(\boldsymbol{r} ; \boldsymbol{R})\left|\nabla_{\boldsymbol{R}_{i}}\right| \Phi_{n}(\boldsymbol{r} ; \boldsymbol{R})\right\rangle}{\mathcal{E}_{m}-\varepsilon_{n}}\right| \ll 1
$$

$\Phi_{m}$ and $\Phi_{n}$ are different electronic states that may couple to one another during the nuclear motion. $\varepsilon_{m}$ and $\varepsilon_{n}$ are corresponding energy eigenvalues. When Massey's criterion is violated due to small energy spacing between electronic states or strong coupling between nuclear and electronic motion, Born-Oppenheimer approximation breaks down. The electronic states will mix with one another during the nuclear motion, and consequently lead to non-adiabatic electronic dynamics. Born-Oppenheimer approximation breakdown has been experimentally observed in many experiments involving exoelectron emission (19), photolysis of molecules (43), vibrationally induced electron emission (44, 45), and chemicurrents (16). Before introducing theories that deal with non-adiabatic phenomena, we first look at a specific non-adiabatic process that is related to this thesis, chemi-current induced by $\mathrm{H}$ atom adsorption on metal surfaces. 

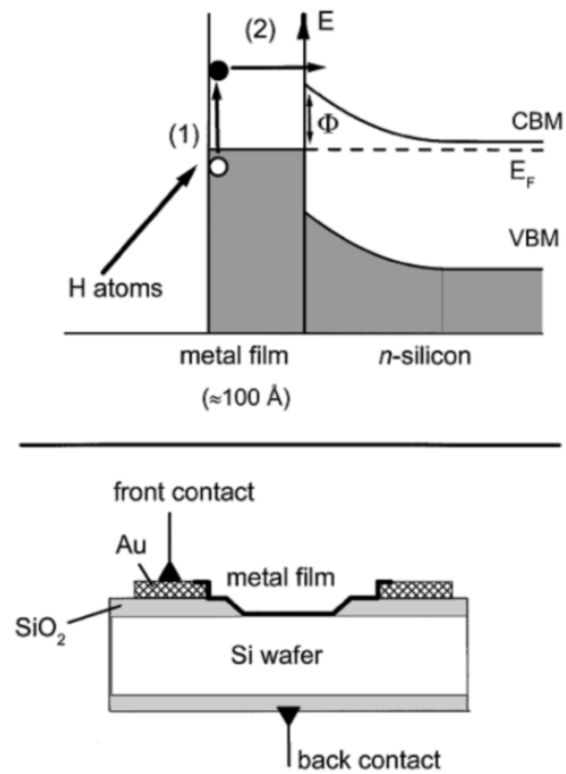

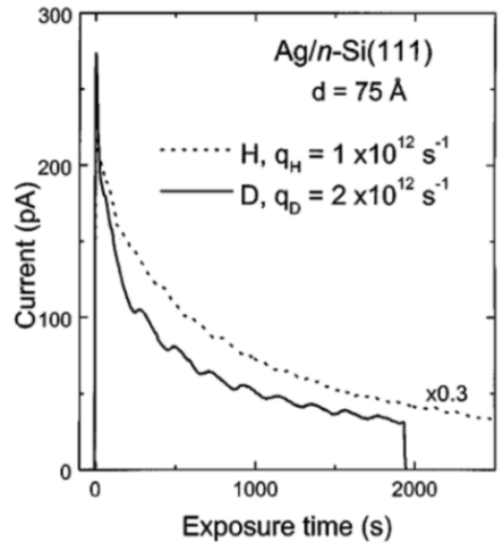

Figure 2.4: Upper left panel: mechanism of chemicurrents induced by $H$ atom adsorption on metal surfaces; (1) hot electrons transport ballistically through the film into the semiconductor; (2) where hot electrons are detected. Lower left: Schematic cut view through the Schottky diode. Right panel: chemicurrent induced by hydrogen and deuterium atom adsorption on a silver surface as a function of exposure time. Reprinted figure with permission from Ref. (18). Copyright (1999) by the American Physical Society.

Electron hole pairs generated by non-adiabatic processes have been detected on devices based on Schottky diodes, metal insulator metal structures (MIM) and metal-insulator-semiconductor (MIS) structures (46-48). The physics behind these devices is shown in the upper left panel of Figure 2.4 using a Schottky diode as an example (18). The transition metal film is evaporated on an n-type Si wafer forming a diode with a Schottky barrier, $\Phi$. The energy of the H-metal bond is around $2 \mathrm{eV}$. If the exothermic chemisorption of $\mathrm{H}$ atoms creates e-h pairs, hot electrons may travel ballistically through the film and cross the barrier. They can be detected as a current which is called chemicurrent. Alternatively, holes can be detected with a p-type semiconductor. The mean free path for electrons and holes is typically on the order of $100 \AA$, which is longer than the film thickness. The barrier ranges from $0.2 \mathrm{eV}$ to more than $1 \mathrm{eV}$. This guarantees the detection of low energy holes and electrons whose energy are not large enough to overcome the work function and escape from the metal. 
The right panel of figure 2.4 shows chemicurrent measured from hydrogen and deuterium atoms adsorption on a silver surface. The chemicurrent transient represents the occupation of empty sites by atoms. After exposure of the surface to an atomic beam, the initial peak current is followed by decay in the current amplitude as the surface coverage increases and the number of available vacant sites decreases. The data indicates a significant isotope effect. The exponential decay and the final steady state can be modeled by the competition of $\mathrm{H}$ atom adsorption and abstraction. The adsorption and abstraction are assumed to be governed by the LangmuirHinshelwood and Eley-Rideal mechanisms, respectively. From the decay time constant, the cross section for adsorption and abstraction can be deduced to be on the order of $10^{-16} \mathrm{~cm}^{2}$.

Several theoretical methods have been developed to include non-adiabatic excitations, including the Newns-Anderson model (49), independent electron surface hopping (IESH) (50, 51), and electronic friction theory $(52,53)$. Here, I will focus on electronic friction theory. The description follows formalism given by Holloway et al. which was used to explain the chemicurrent induced by $\mathrm{H}$ atom adsorption on metal surfaces (23). Models based on electronic friction theory employ a nearly adiabatic approximation, and treat the low energy electron hole pair excitations as a classical velocity-dependent friction force. After the appropriate friction coefficients have been obtained, the non-adiabatic excitations are incorporated into the classical trajectory simulations by adding a damping term. To simplify the discussion, we restrict the adsorbed atom to a one-dimensional coordinate, $z(t)$ and we hold the surface atoms frozen in their equilibrium positions. The instantaneous energy expectation value of the many-electron system in the presence of an adsorbate atom is:

$$
E(t)=\left\langle\Phi(t)\left|H_{0}+V_{e x t}(t)\right| \Phi(t)\right\rangle
$$

$H_{0}$ is the many-electron Hamiltonian, $V_{\text {ext }}(t)$ is the time-dependent interaction of electrons with the moving nucleus, $\Phi(t)$ is the many-electron wave function satisfying the time dependent Schrödinger equation with a Hamiltonian of $H=H_{0}+V_{\text {ext }}(t)$. The interaction term $V_{\text {ext }}(t)$ can be expressed as:

$$
V_{\text {ext }}(t)=\int d \boldsymbol{r} V_{\text {ext }}(\boldsymbol{r}, z(t)) n(\boldsymbol{r})
$$


$V_{\text {ext }}(\boldsymbol{r}, z(t))$ is the Column interaction potential between the electron and the nucleus and $n(\boldsymbol{r})$ is the electron density operator. The non-adiabatic energy is $E_{n a}(t)=E(t)-E_{0}(t)$, where $E_{0}(t)$ is the instantaneous ground state energy. Combining Eq. 2.14 and Eq. 2.15, we obtain the rate of non-adiabatic energy transfer as:

$$
\dot{E}_{n a}(t)=\dot{z}(t) \int d \boldsymbol{r} \frac{d V_{\text {ext }}(\boldsymbol{r}, z(t))}{d z} \delta n(\boldsymbol{r}, z)
$$

$\delta n(\boldsymbol{r}, z)=n(\boldsymbol{r}, z)-n_{0}(\boldsymbol{r}, z)$ is the deviation of instantaneous electron density from the ground state electron density. Under "nearly adiabatic" approximation, the small change $\delta n(\boldsymbol{r}, z)$ can be obtained by using a linear response of the electron system to the perturbation from the adsorbed atom. The non-adiabatic energy transfer rate can then be expressed as:

$$
\dot{E}_{n a}(t)=\dot{z}(t) \int_{-\infty}^{t} d t^{\prime} \Lambda\left(t-t^{\prime} ; z(t)\right) z\left(t^{\prime}\right)
$$

$\Lambda\left(t-t^{\prime} ; z(t)\right)$ is the memory function deduced from the linear response theory. By Fourier transforming the time domain memory function $\Lambda\left(t-t^{\prime} ; z(t)\right)$ into frequency domain $\Lambda(\omega ; z)$ and using the low frequency limit of the response $\Lambda(\omega ; z)=i \eta(z) \omega$, we can rewrite Eq. 2.17 as:

$$
\dot{E}_{n a}(t)=\eta(z(t))(\dot{z}(t))^{2}
$$

By simply applying energy conservation considerations, we can express the non-adiabatic excitation as a friction force $F=\eta(z(t)) \times \dot{z}(t)$. The position-dependent parameter $\eta(z(t))$ can be viewed as the fiction coefficient. The Newton equation for the classical trajectory simulations is expressed as:

$$
M_{i} \ddot{z}(t)=-\nabla_{z} \mathcal{E}(\boldsymbol{R}, z)-\eta(z(t)) \dot{z}(t)
$$

Several self-consistent methods can be used to determine the friction coefficient $(23,54)$. In the formalism of Holloway et al., time dependent density functional theory (TDDFT) is used to calculate friction coefficient. This has the advantage that the friction coefficient $\eta(z(t))$ can be related to the electron-hole pair energy distribution $P_{S}(\omega)$ under a forced oscillator model (FOM). In the forced oscillator model, the oscillators are electron-hole pairs, and they are driven by the 
changing potential of the adsorbate. Holloway et al. gave the relation between the energy distribution and the friction coefficient $\eta(z(t))$ as:

$$
P_{S}(\omega)=\sum_{\sigma} \frac{1}{\pi \hbar \omega}\left|\int_{-\infty}^{\infty} \eta_{\sigma}^{\frac{1}{2}}(z) \dot{z}(t) e^{-i \omega t} d t\right|^{2}
$$

$\sigma$ indicates the spin state. From the energy distribution, the number of electrons that have enough energy to cross the Schottky barrier can be calculated.

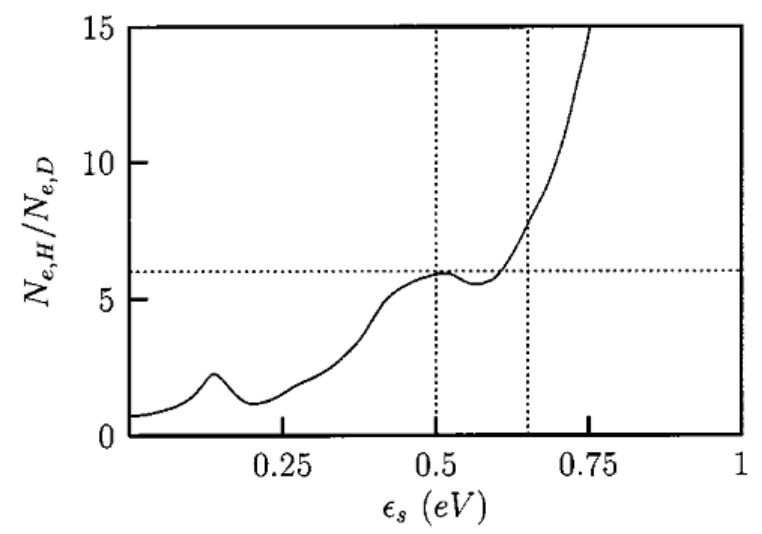

Figure 2.5: the calculated ratio of chemicurrent arising from $H$ and $D$ atoms adsorption on top site of $\mathrm{Cu}(111)$. The horizontal dashed line indicates the measured chemicurrent ratio. The vertical dashed lines indicate the range of Schottky barrier height for the experiment. Reprinted from Ref. (23), with the permission of AIP publishing.

Figure 2.5 shows the comparison of simulated and measured chemicurrent ratios due to the adsorption of $\mathrm{H}$ and $\mathrm{D}$ atoms on the $\mathrm{Cu}$ surface. Due to the velocity difference, $\mathrm{H}$ atoms adsorption induces more hot electrons than $\mathrm{D}$ atoms do. The experimental setup was introduced earlier in this section. The $\mathrm{x}$ axis shows the barrier height of the Schottky diode, and the y axis represents the ratio of hot electrons that can pass the Schottky barrier for $\mathrm{H}$ and $\mathrm{D}$ atoms adsorption. Solid black line represents the theoretically calculated results. The horizontal dashed line shows the measured ratio. The vertical dashed lines indicate the range of Schottky barrier heights found in the experiments (18). Reasonable agreement is found between experiment and theory, which supports the validation of electronic friction theory in describing non-adiabatic ehps excitations. 


\section{Experimental Methods and Setup}

In this chapter, I will present the experimental methods and setup used in this thesis. Two widely known techniques in gas phase experiments, atom beam generation via photolysis and Rydberg atom tagging time-of-flight, are combined in our surface scattering study. $\mathrm{H}$ atom beams with narrow energy distributions are generated via laser photodissociation of hydrogen halide molecules and scattered from a well-defined single crystal surface. The time-of-flight spectrum of the scattered $\mathrm{H}$ atoms is measured using the Rydberg atom tagging method. A complete set of angular and energy distribution is measured with a rotatable detector at high angular resolution $\left(3^{\circ}\right)$. The single crystal sample is mounted on a home-designed 6-axis manipulator. The surface can be cleaned and characterized via standard techniques including ion sputtering, electron bombardment heating, low energy electron diffraction (LEED) and Auger electron spectroscopy.

\subsection{Mono-energetic narrow energy distribution H/D atom beams}

Hydrogen atom beams with kinetic energies in the range of chemical interest are usually generated via dissociation of molecules. Popular methods include discharge, thermal cracking and photodissociation. Among these methods, photodissociation has the advantage of generating atom beams with tunable energy and narrow velocity spread. A molecule containing hydrogen is excited to a repulsive state via photon absorption. The repulsive state dissociates to generate $\mathrm{H}$ atoms. Translational energy of the hydrogen atom is calculated from energy (Eq. 3.1) and momentum (Eq. 3.2) conservation.

$$
\begin{gathered}
E_{\text {int }}^{0}+\hbar \omega=E_{0}+E_{\text {tran }}^{H}+E_{\text {tran }}^{X}+E_{\text {int }}^{X} \\
m_{H} v_{H}=m_{X} v_{X} \text { or } \frac{E_{\text {tran }}^{H}}{m_{H}}=\frac{E_{\text {tran }}^{X}}{m_{X}}
\end{gathered}
$$

Here, $E_{\text {tran }}^{0}$ indicates the initial internal energy in the molecule (possibly including electronic, vibrational and rotational energies). $\hbar \omega$ represents the energy of the absorbed photon. $E_{0}$ indicates the dissociation energy of the molecule. $E_{\text {tran }}^{H}$ and $E_{\text {tran }}^{X}$ are kinetic energies of the $\mathrm{H}$ atom and the other photofragment. $E_{i n t}^{X}$ indicates the possible internal excitation in the photofragment $\mathrm{X}$ (possibly including electronic, vibrational and rotational energies). $m_{H}$ and $m_{X}$ 
are the masses of the two fragments, and $v_{H}$ and $v_{X}$ represent the recoiling velocities of the two fragments.

In this thesis, narrow energy bandwidth $\mathrm{H}$ atom beams are generated via photolysis of supersonically expanded hydrogen halide molecular beams using ultraviolet (UV) and vacuum ultraviolet (VUV) lasers. Translational energy of the atom beam can be tuned by the photolysis laser wavelength. The supersonic expansion ensures that the hydrogen halide molecular beams have low rotational temperatures, reducing the energy spread of the $E_{\text {int }}^{0}$ term in Eq.3.1. Hydrogen halide dissociation only produces two atomic photofragments, reducing the energy spread of the $E_{\text {int }}^{X}$ term. Another advantage of using hydrogen halides is that atomic masses of the $\mathrm{I}$ and $\mathrm{Br}$ atoms are much larger than that of the $\mathrm{H}$ atom, which means that most of the available energy is converted to the translational energy of the $\mathrm{H}$ atom.

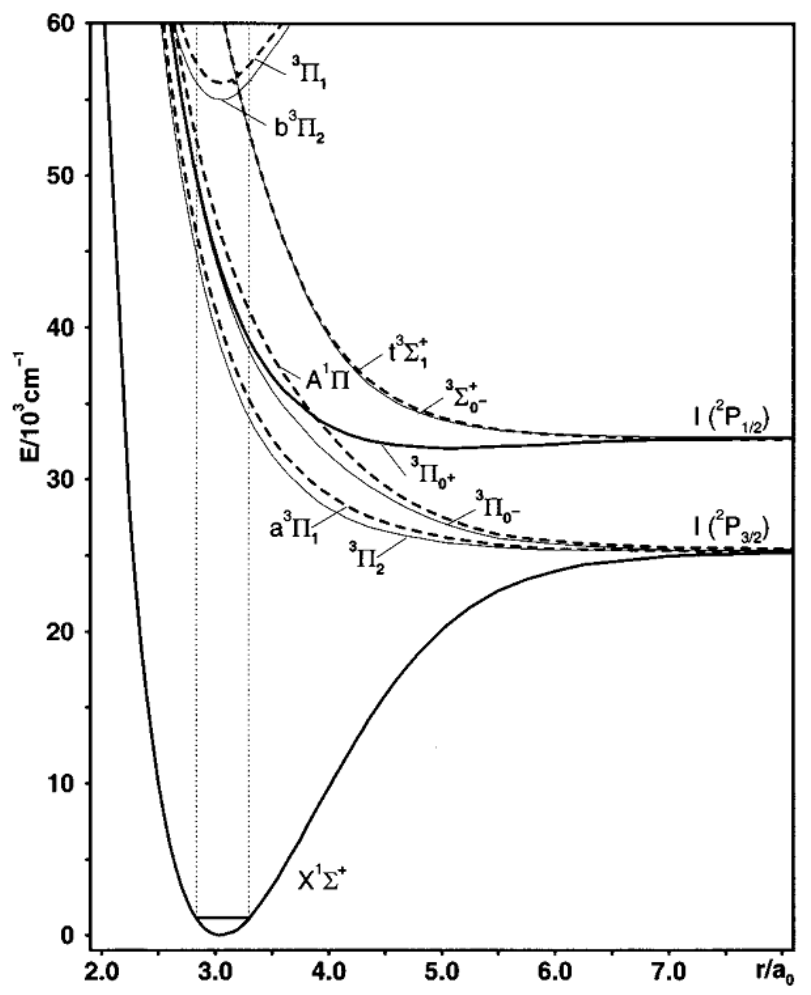

Figure 3.1: Calculated potential energy curves for the low lying states of hydrogen iodide. Reprinted from Ref. (55), with the permission of AIP publishing.

Figure 3.1 shows the calculated potential energy curves for low lying states of hydrogen iodide (HI) (55). Photodissociation of HI using excimer lasers involves two dissociation channels. One 
channel generates ground state ${ }^{2} \mathrm{P}_{3 / 2}$ iodine atom (I), the other produces excited state ${ }^{2} \mathrm{P}_{1 / 2}$ iodine atom $\left(\mathrm{I}^{*}\right)$. The energy difference between $\mathrm{I}$ and $\mathrm{I}^{*}$ state is $7603 \mathrm{~cm}^{-1}$. In this thesis, the shortest laser wavelength used is $193 \mathrm{~nm}$ (around $51800 \mathrm{~cm}^{-1}$ ), indicating the allowed transitions are from ground $\mathrm{X}^{1} \Sigma^{+}$state to excited ${ }^{1} \Pi_{1},{ }^{3} \Pi_{1}$ and ${ }^{3} \Pi_{0}{ }^{+}$states. Excitations to ${ }^{1} \Pi_{1}$ and ${ }^{3} \Pi_{1}$ states are perpendicular transitions $(\Delta \Omega= \pm 1)$ and correlate to ground state I. Excitations to ${ }^{3} \Pi_{0}{ }^{+}$state is parallel transition $(\Delta \Omega=0)$ and correlate to excited state $I^{*}$. The angular distribution of photodissociation products depends on the angle $\theta$ between the laser polarization and the recoil velocity and has the form (56):

$$
I(\theta)=\frac{1}{4 \pi}\left[1+\beta P_{2}(\cos (\theta))\right]
$$

Here, $\beta$ is the so called spatial anisotropy parameter. It equals 2 when the dissociation follows a pure parallel transition and to -1 when the fragmentation follows a pure perpendicular transition for prompt dissociation of diatomic molecules. $P_{2}(\cos (\theta))=\frac{1}{2}\left(3 \cos (\theta)^{2}-1\right)$ is the second order Legendre polynomial. The total quantum yield, branching ratio $\left(\mathrm{P}(\mathrm{I}) / \mathrm{P}\left(\mathrm{I}^{*}\right)\right)$ and the fragment angular distribution of hydrogen halide molecule photodissociation at different laser wavelengths are described in detail in ref (56).

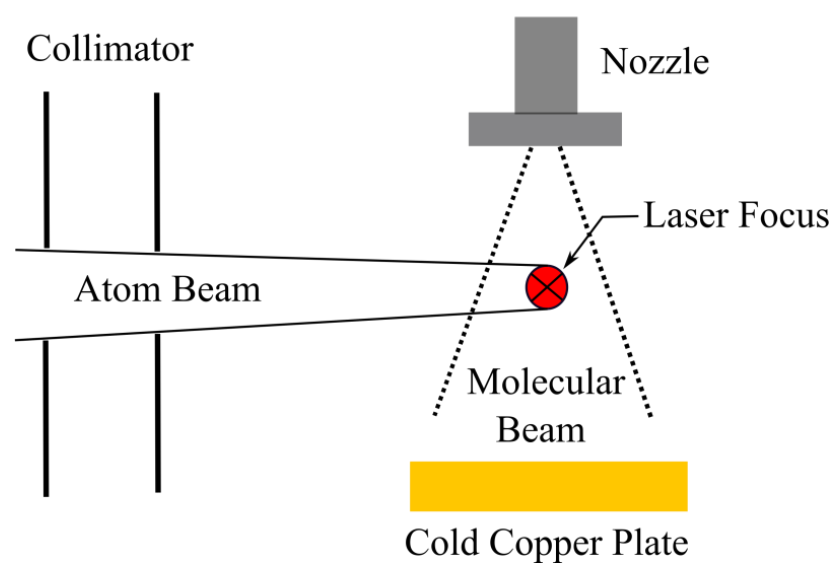

Figure 3.2: Illustration of the laser photolysis geometry used in this thesis.

Figure 3.2 shows the geometry of the laser photolysis used in this thesis. The supersonic hydrogen halide molecular beam is generated by a pulsed general valve and then crossed by a pulsed photolysis laser beam (propagating into the page). The supersonic expansion takes place 
in a high vacuum source chamber $\left(1 \times 10^{-6} \mathrm{mbar}\right.$ when nozzle is running). A small portion of the photofragment $\mathrm{H}$ atoms fly through two collimating apertures in the chamber wall in a direction perpendicular to the molecular beam and photolysis laser to form the $\mathrm{H}$ atom beam directed into the UHV scattering chamber. A liquid nitrogen cooled copper plate is placed directly under the general valve to freeze the hydrogen halide molecules. This ensures that no hydrogen halide molecule can fly into the UHV chamber and contaminate the surface sample.
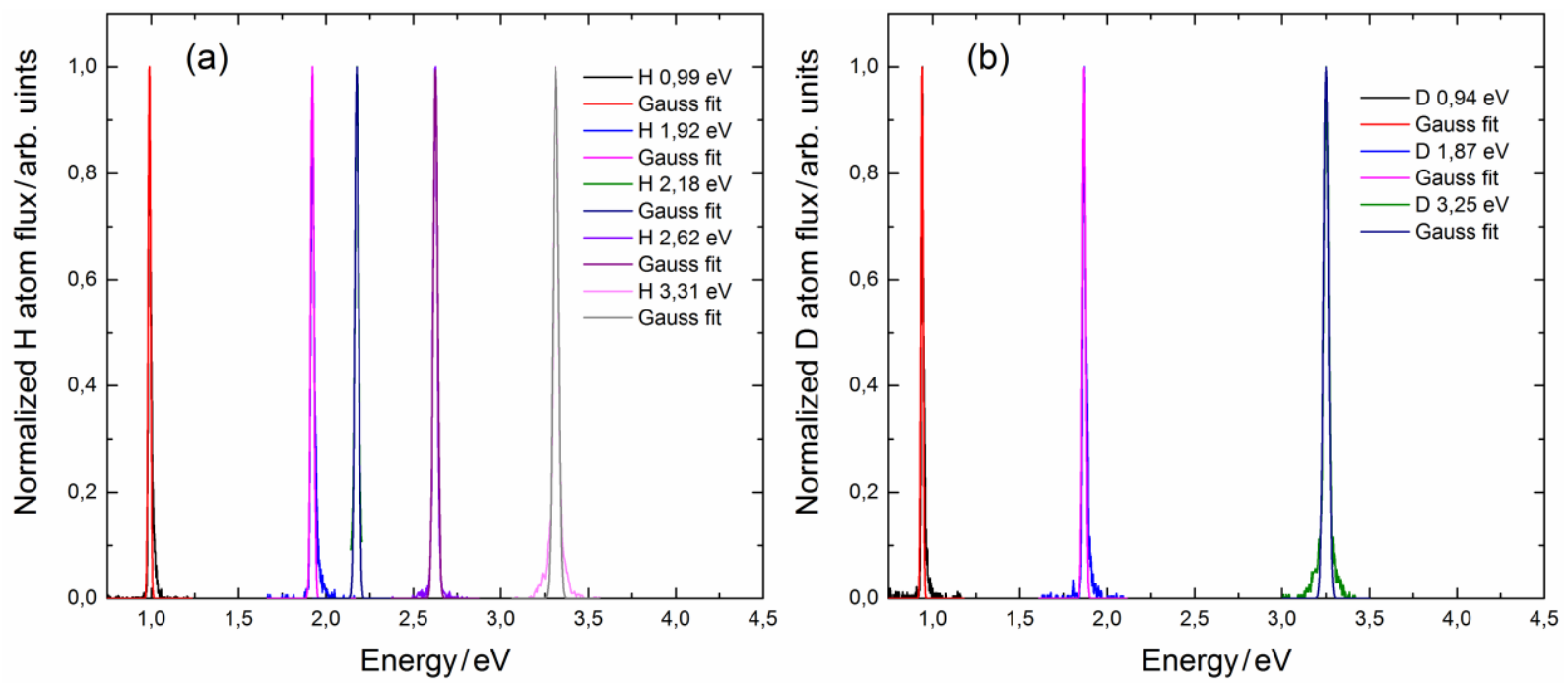

Figure 3.3: Translational energy distribution of the incidence $H$ and $D$ beams

Figure 3.3 shows the observed kinetic energy profile of the incidence beams and a fit of the observed profiles to a Gaussian distribution. The $\mathrm{H}$ and $\mathrm{D}$ atom beams are generated by laser photolysis of supersonic $\mathrm{HI}, \mathrm{HBr}$ or DI molecular beams. Non-polarized $\mathrm{KrF}(248 \mathrm{~nm})$ and $\mathrm{ArF}$ $(193 \mathrm{~nm})$ excimer lasers are used for photolysis. The bandwidth of the excimer laser depends on the type of buffer gas, pressure of the gas mixture, and discharge voltage, and is typically around $0.5 \mathrm{~nm}$. Photolysis of $\mathrm{HI}$ molecules by the $248 \mathrm{~nm}$ output of the $\mathrm{KrF}$ laser generates $\mathrm{H}$ atom beams with energies of $0.99 \mathrm{eV}$ and $1.92 \mathrm{eV}$, corresponding to the two different dissociation product channels, which result in the ground $\left({ }^{2} \mathrm{P}_{3 / 2}\right)$ state and $\left({ }^{2} \mathrm{P}_{1 / 2}\right)$ excited state of the I atoms, respectively. Photolysis of $\mathrm{HBr}$ molecule by $\mathrm{ArF}$ laser produces $\mathrm{H}$ atom beams with energies of $2.18 \mathrm{eV}$ and $2.62 \mathrm{eV}$. Photolysis of $\mathrm{HI}$ molecules using ArF laser only provides $\mathrm{H}$ atom with $3.31 \mathrm{eV}$ translational energy $\left({ }^{2} \mathrm{P}_{3 / 2}\right.$ ground state $\mathrm{I}$ atom). The $\mathrm{D}$ atom energies produced by photolysis of DI molecules are $0.94 \mathrm{eV}, 1.87 \mathrm{eV}$ and $3.25 \mathrm{eV}$. FWHMs for the atom beams are 10-20 meV depending on the incidence energies. 


\subsection{Rydberg atom tagging time-of-flight and VUV generation}

For the experiments presented in this thesis, translational energy distribution of the hydrogen atoms scattered back from the single crystal surface is measured via time-of-flight. Figure 3.4 illustrates the time-of-flight measurement geometry. Incidence beam, scattered beam and surface normal are in the plane of the page. The excitation laser beams (red circle) propagate perpendicularly to the plane of the page. The scattered hydrogen atoms are first tagged by laser excitation, and the tagging moment is defined as time zero. The tagged atoms fly a distance $\mathrm{L}$ and are detected subsequently. The measured arrival time distribution $F(t)$ is converted to translational energy distribution by multiplying a Jacobian correction factor and a density-to-flux correction factor. Since the pulsed laser only excites $\mathrm{H}$ atoms in a certain volume (laser focus volume), the detected signal is proportional to the atom density. Multiplying a correction factor $v$ (speed), the density signal is converted to flux signal.

$$
\begin{gathered}
f_{\text {den }}(E)=\frac{d t}{d E} \times F(t) \propto t^{3} \times F(t) \\
f_{\text {flux }}(E)=v \times f_{\text {den }}(E) \propto \frac{f_{d e n}(E)}{t} \\
f_{\text {flux }}(E) \propto t^{2} \times F(t)
\end{gathered}
$$

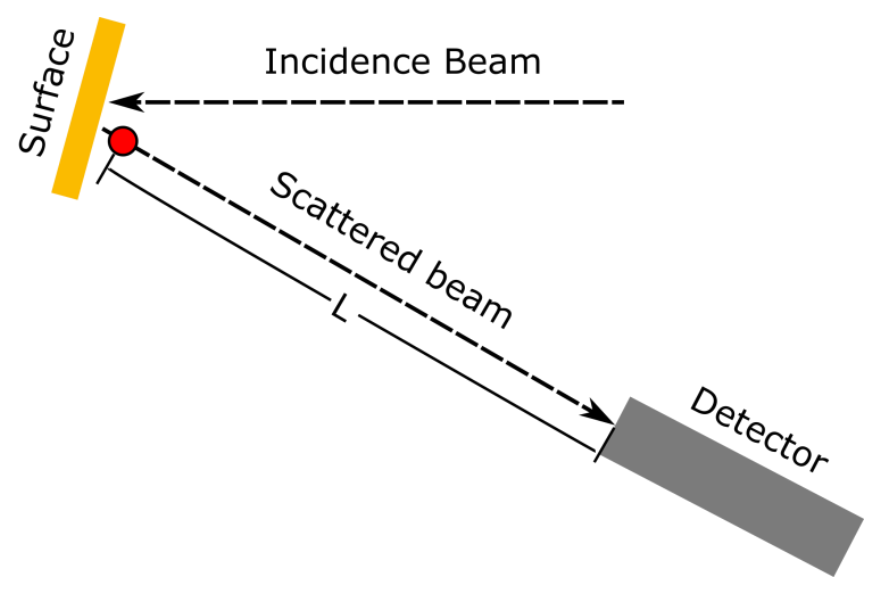

Figure 3.4: illustration of the time-of-flight measurement geometry. 
Hydrogen atom Rydberg excitation is used to tag the time zero (57). As shown in Figure 3.5, the atom is excited to a high Rydberg state via a two-step transition (shown as the right excitation scheme in Figure 3.5). First, the ground state hydrogen atom is excited to the $2 \mathrm{P}$ state via the Lyman alpha transition $(121.6 \mathrm{~nm})$. Then, another UV photon (around $365 \mathrm{~nm}$ ) subsequently excites the $2 \mathrm{P}$ hydrogen atom to a high Rydberg state, whose principle quantum number $\mathrm{n}$ ranges from 30 to 90 depending on the energy of the UV photon (in this thesis, $n=34$ is used). The neutral Rydberg atom flies a distance $\mathrm{L}$ and is then field ionized and detected by a $\mathrm{Z}$ stacked micro-channel plate (MCP) assembly. The Rydberg atom $(n=34)$ lies only several meV below the ionization threshold and can be field ionized in electric fields above $1 \mathrm{kV} / \mathrm{cm}$. Compared to the commonly used REMPI scheme (resonant multi-photon ionization, shown as the left excitation scheme in Figure 3.5), Rydberg atom tagging significantly improves the translational energy resolution of the time-of-flight method. First, the atoms are neutral until they reach the detector. Neither space charge effects nor stray fields limit the resolution. Second, lifetimes of the Rydberg atoms are quite long (on the order of several tens of $\mu$ s for principle quantum number $n=34$ ). Applying an electric field in the excitation region could extend the lifetime to the millisecond range, due to parabolic state formation. This long lifetime enables the use of long flight distance, providing high resolution in the translational energy measurement.

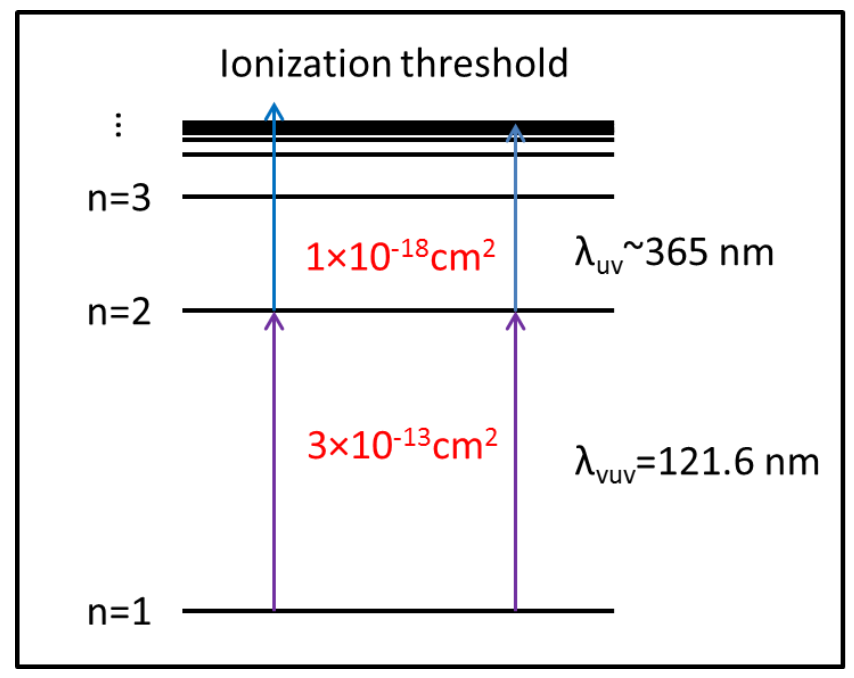

Figure 3.5: Excitation scheme for hydrogen atoms. Left scheme: REMPI (resonant multi-photon ionization). Right scheme: Rydberg atom tagging. The red numbers indicate the absorption cross sections for the corresponding excitations. 
Figure 3.6 shows the geometry of the laser excitation. All the laser beams and the $\mathrm{H}$ atom beam are in the same plane. The $121.6 \mathrm{~nm}$ and $365 \mathrm{~nm}$ excitation laser beams counter propagate and are focused on to the $\mathrm{H}$ atom beam at the same spot. The VUV laser beam is focused by a LiF lens, which has low absorption at $121.6 \mathrm{~nm}$. The $121.6 \mathrm{~nm}$ VUV radiation is generated from a $212.5 \mathrm{~nm}$ UV laser and an $845 \mathrm{~nm}$ infrared laser via nonlinear four-wave mixing. Figure 3.7 shows the scheme of the non-linear four-wave mixing (58). A $212 \mathrm{~nm}$ UV laser pulse and an 845 $\mathrm{nm}$ infrared laser pulse are focused into a cell containing a $\mathrm{Kr}$ and $\mathrm{Ar}$ gas mixture (partial pressure ratio $1: 3$, total pressure around $100 \mathrm{mbar}$ ). The $\mathrm{Kr}$ atom is resonantly excited into a $4 p^{5} 5 p$ state by absorbing two $212.5 \mathrm{~nm}$ UV photons. The VUV photon is produced via the $4 \mathrm{p}^{5} 5 \mathrm{p}$ state relaxation induced by an infrared $845 \mathrm{~nm}$ photon. The wavelength of the VUV photon can be tuned by tuning the infrared laser. The frequency relation obeys the energy conservation:

$$
\hbar \omega_{V U V}=2 \times \hbar \omega_{U V}-\hbar \omega_{i n f r a}
$$

The Ar gas is used to fulfill the phase matching condition:

$$
k_{V U V}=2 \times k_{U V}-k_{\text {infra }}
$$

The power of the $121.6 \mathrm{~nm}$ VUV laser follows:

$$
P_{V U V} \propto P_{U V}^{2} P_{\text {infra }} \chi^{2}
$$

Here $\chi$ is the third order nonlinear susceptibility. The power of the VUV laser also depends on the gas mixture pressure which is experimentally optimized.

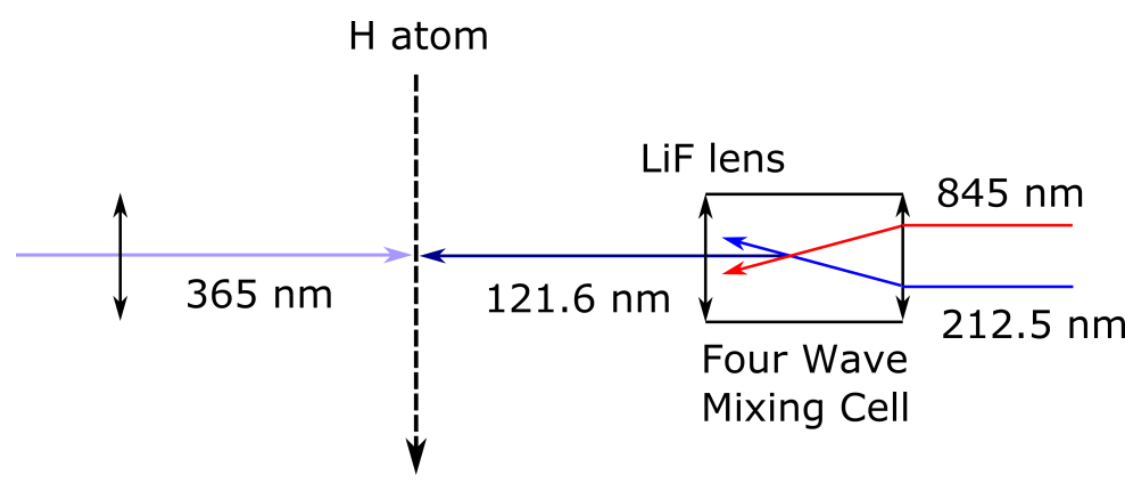

Figure 3.6: Geometry of the laser excitation. 


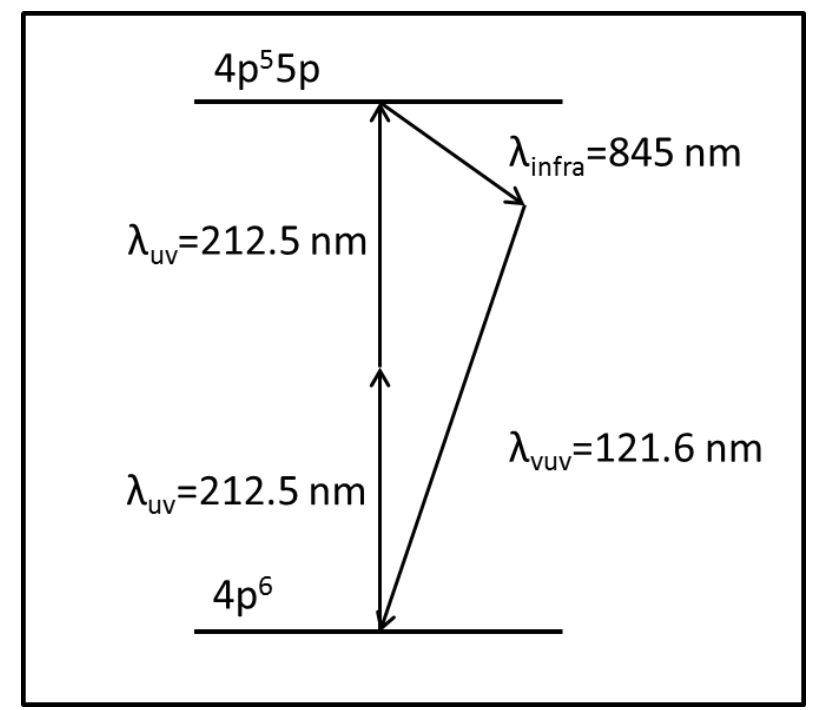

Figure 3.7: Illustration of the $121.6 \mathrm{~nm}$ VUV generation from four-wave mixing.

\subsection{Surface preparation and characterization}

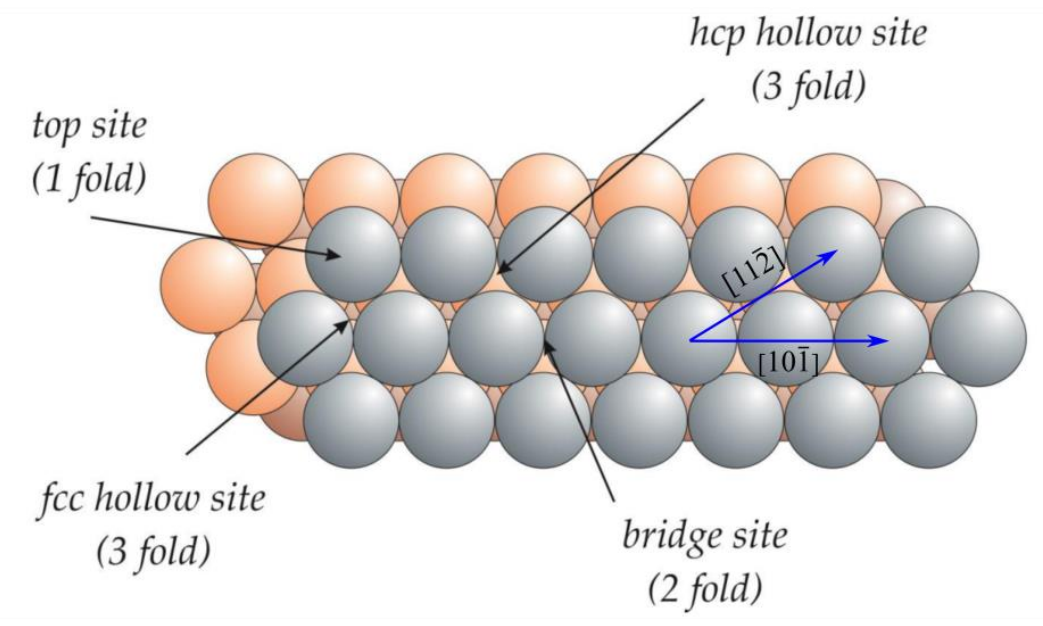

Figure 3.8: Illustration for the (111) surface of fcc metals.

In this thesis, the (111) surface of fcc (face centered cubic) metals (Au and Pt) are used. Figure 3.8 shows the structure of the (111) surface of an fcc metal. The fcc (111) surface is one of the most densely packed atomic planes. It has three-fold symmetry and offers 4 different kinds of adsorption site as shown in the figure. Two different types of hollow sites exist on fcc (111) surface. The hcp hollow sites sit on top of Au atoms in the second atomic plane, while the fcc 


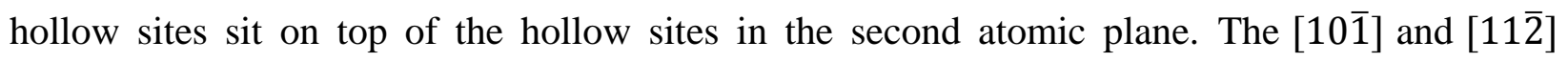
surface directions are shown as blue arrows in the figure.

Atoms on the fcc (111) surface plane have fewer neighbors than those in the bulk, indicating an increase of the surface energy. Therefore, the surface atoms tend to rearrange their positions to minimize the energy, leading to a deviation from the perfect (111) surface structure. The rearrangement of atom positions is called surface reconstruction. Figure 3.9 shows the wellknown herring-bone-like reconstruction of the $\mathrm{Au}$ (111) surface. On a nanometer scale, it forms a $22 \times \sqrt{3}$ structure, by compressing 23 atoms in 22 lattice spacing in the [11ㅎ] surface direction. On a long-range hundred nanometer scale, it forms a herring-bone-like structure as shown in Figure 3.9.

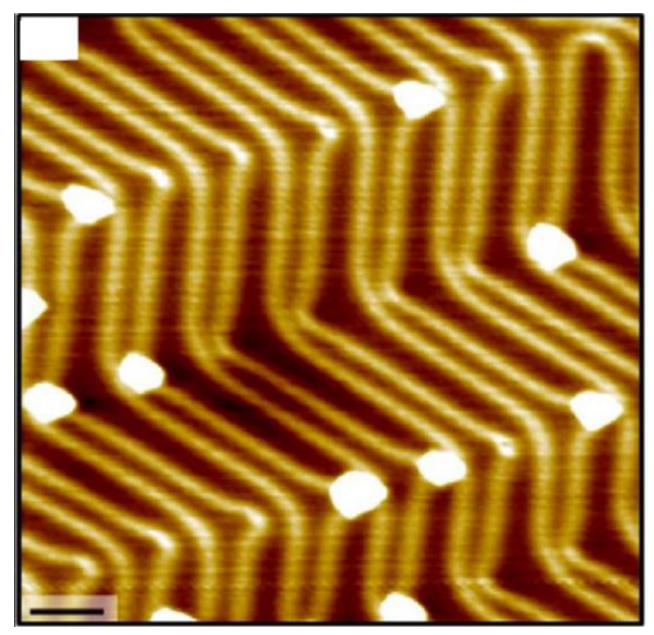

Figure 3.9: STM image of Au(111) $22 \times \sqrt{3}$ herring-bone-like reconstruction. Scale bar equals $6 \mathrm{~nm}$. Reprinted from Ref. (59). Copyright (2012), with the permission from Elsevier.

Surface samples are cleaned via standard ion sputtering and subsequent high temperature annealing. For $\mathrm{Au}(111)$, the surface is usually sputtered with $3 \mathrm{kV}$ Ar ion for half an hour (ion current $30 \mu \mathrm{A}$ ) and then annealed at $800{ }^{\circ} \mathrm{C}$ for another half an hour via electron bombardment. For $\operatorname{Pt}(111)$, the annealing temperature is raised to $900{ }^{\circ} \mathrm{C}$. If the procedure described above cannot produce clean $\mathrm{Pt}(111)$ surface, annealing in oxygen atmosphere (partial pressure about $1 \times 10^{6} \mathrm{mbar}$ ) at $650{ }^{\circ} \mathrm{C}$ is used in between the sputtering and annealing at $900{ }^{\circ} \mathrm{C}$. The cleanliness and surface structure can be checked by low energy electron diffraction (LEED) and Auger electron spectroscopy, which will be introduced later. Figure 3.10 shows a sketch of the electron 
bombardment heating. A tungsten filament several millimeters behind the surface sample is heated with 3-5 A current. Electrons emitted by the filament are accelerated to the surface sample which is held at high voltage $(600 \sim 1000 \mathrm{~V})$, and the emission current can be tuned between 10 to $30 \mathrm{~mA}$ by changing the current through the filament. This provides several tens of watts of heating power and easily heats the surface to $1000{ }^{\circ} \mathrm{C}$.

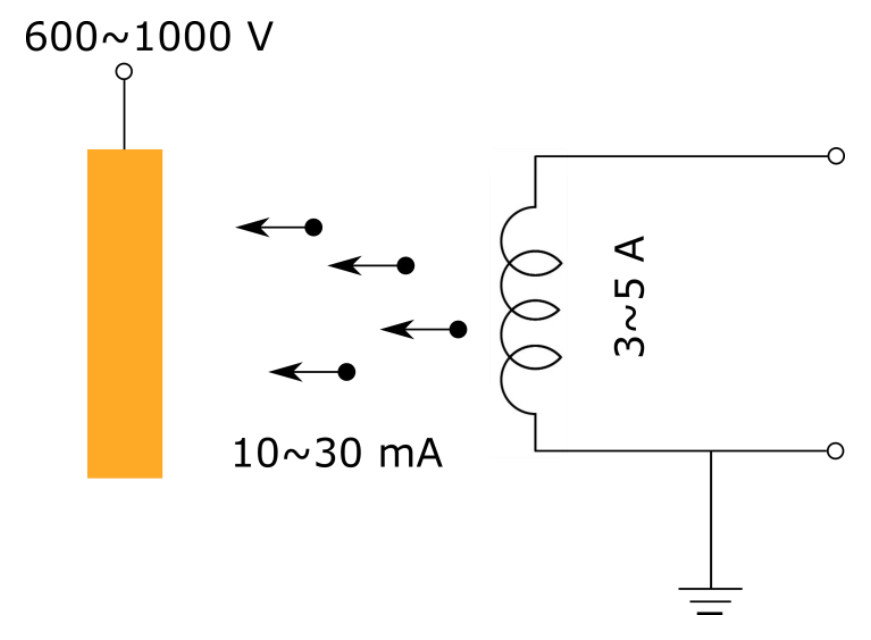

Figure 3.10: Illustration of the electron bombardment heating.

The quality of the surface structure is checked by low energy electron diffraction (LEED). De Broglie wavelengths of electrons with energies about $100 \mathrm{meV}$ are around $1 \AA$, which is on the order of lattice constants of metal crystals. Coherent electron beams shooting at well-defined crystal surface are diffracted and form patterns reflecting the surface structure (reciprocal lattice of the surface). Figure 3.11 shows the LEED measurement on $\operatorname{Pt}(111)$ surface. Clear six-fold symmetry indicates a good quality (111) surface. 


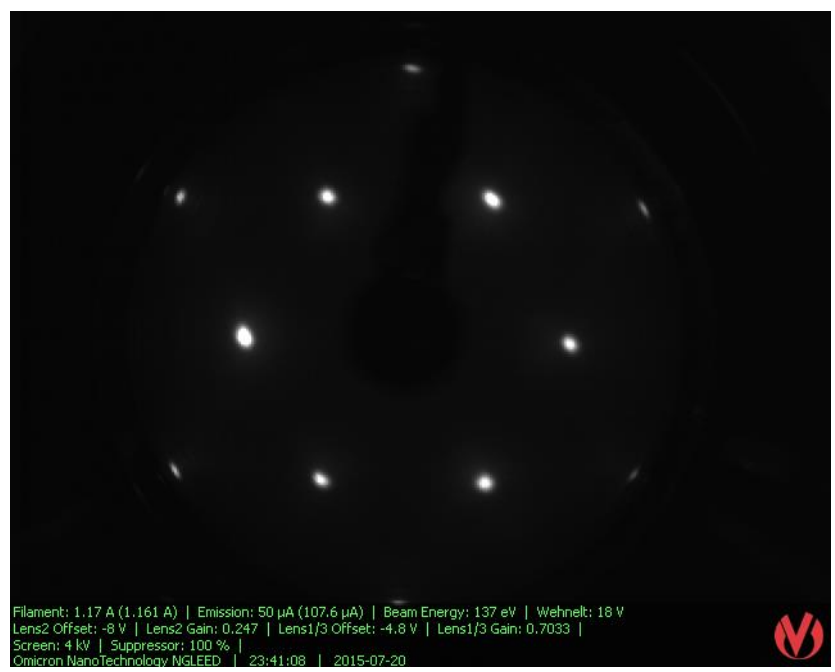

Figure 3.11: LEED pattern of Pt(111) surface.

Surface cleanliness is checked by the Auger electron spectroscopy. High energy electrons (3 5 $\mathrm{kV}$ ) are shot onto the surface, and core electrons of the surface atoms including impurities are kicked out. An electron from a higher energy level subsequently falls into the core vacancy, and another electron is simultaneously released. This is the so-called Auger effect and the released electron is called the Auger electron. The translational energy of the Auger electron reflects the difference between energy levels and is atomic-species specific. The auger electron spectrum can be used to quantitatively analyze the surface composition. The cleanliness of the surface is confirmed by the condition that no Auger electron signal from impurity atoms is seen. Figure 3.12 shows the Auger electron spectra of clean and dirty Pt(111) surfaces. The peaks of impurity $\mathrm{C}$ and $\mathrm{Ca}$ atoms are marked by dashed lines. All the other peaks are from Pt. After annealing the surface in an $\mathrm{O}_{2}$ atmosphere, the $\mathrm{C}$ atom impurities are removed. After two cycles of sputtering and annealing, the $\mathrm{Ca}$ atom impurities are removed, and we get a clean $\operatorname{Pt}(111)$ surface. The surface cleanliness is further confirmed by the scattering signals, which is also sensitive to impurities. 


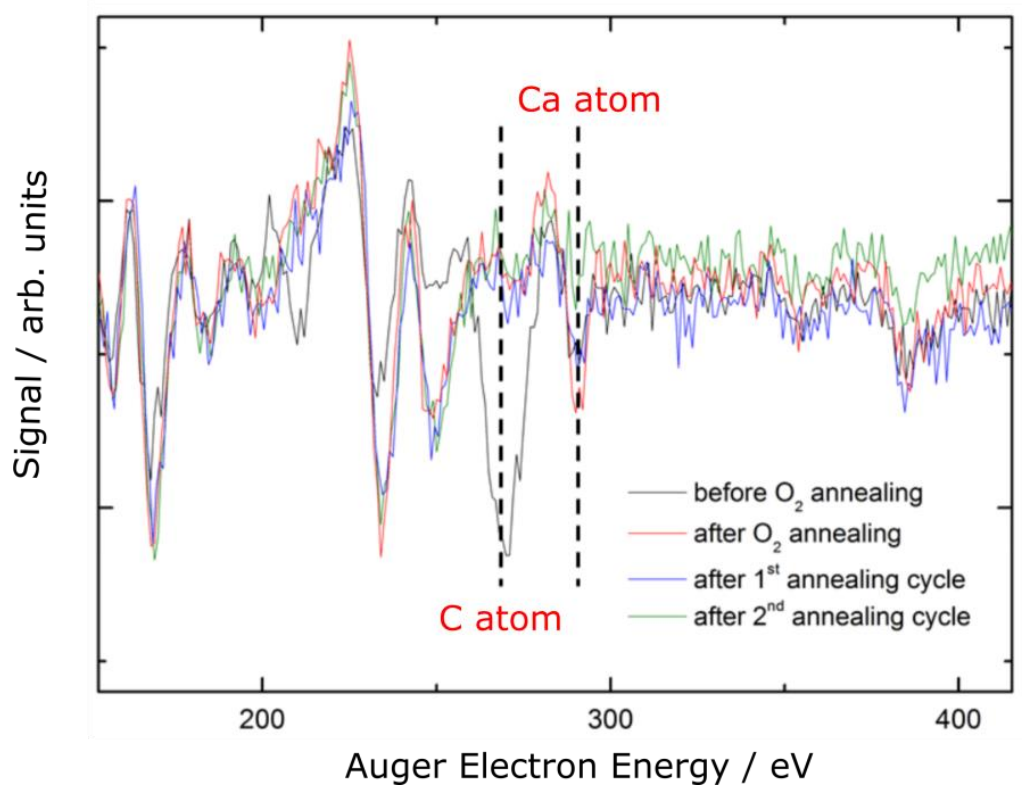

Figure 3.12: Auger electron spectra of clean and dirty Pt(111) surface.

\subsection{Laser system, experimental geometry and timing}

\subsubsection{Laser system}

Figure 3.13 shows the laser system used in the experiments described in this thesis. As discussed in section 3.1 and 3.2, the laser system can be divided into two sub-systems: tagging laser system and photolysis laser system. The tagging laser system generates the $121.6 \mathrm{~nm}$ VUV and $365 \mathrm{~nm}$ UV lasers to excite the $\mathrm{H}$ atom to a high $\mathrm{n}$ Rydberg state. The $532 \mathrm{~nm}$ second order harmonic output from a YAG laser pumps three dye lasers. A red dye laser using the DCM dye is pumped by $130 \mathrm{~mJ}$ of $532 \mathrm{~nm}$ radiation and produces about $60 \mathrm{~mJ}$ of $673.5 \mathrm{~nm}$ output. The $673.5 \mathrm{~nm}$ is further frequency tripled to produce $2 \sim 3 \mathrm{~mJ}$ of $212.5 \mathrm{~nm}$ output. An infrared dye laser using a mixture of LDS821 and LDS827 is pumped by $120 \mathrm{~mJ} 532 \mathrm{~nm}$ radiation and produces about 12 $\mathrm{mJ}$ of $845 \mathrm{~nm}$ radiation. The $212.5 \mathrm{~nm}$ and $845 \mathrm{~nm}$ laser are focused into the four-wave mixing cell to generate the $121.6 \mathrm{~nm}$ Lyman $\alpha$ VUV radiation. A green dye laser is pumped by $120 \mathrm{~mJ}$ of $532 \mathrm{~nm}$ radiation and provides about $25 \mathrm{~mJ}$ of $555 \mathrm{~nm}$ output. The $555 \mathrm{~nm}$ laser is further mixed with the $1064 \mathrm{~nm}$ fundamental YAG output to produce $12 \mathrm{~mJ}$ of $365 \mathrm{~nm}$ radiation via sum frequency generation. 


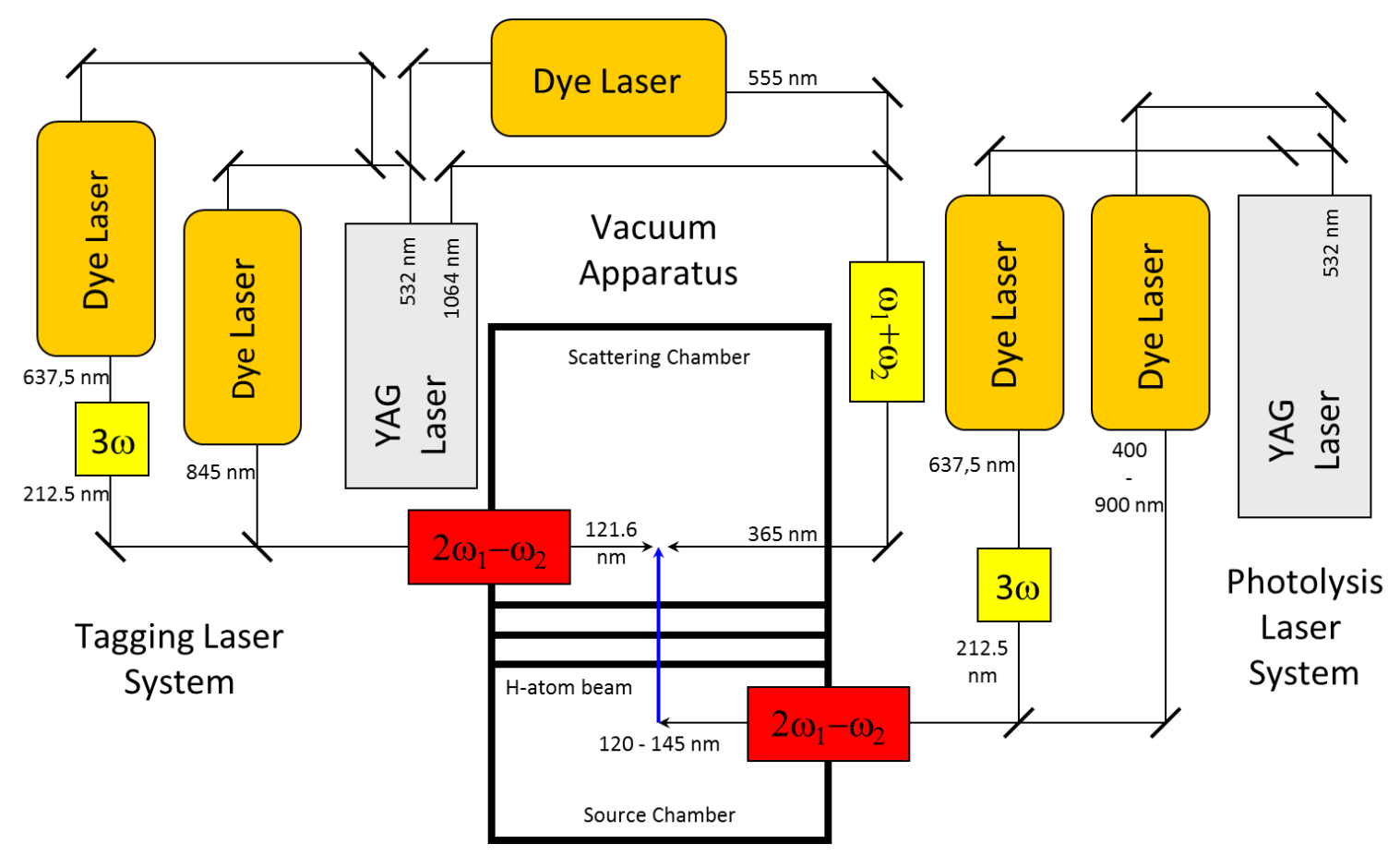

Figure 3.13: Illustration of the laser system.

The photolysis laser system has three versions. The first version shown in Figure 3.13 is used to generate high translational energy $\mathrm{H}$ atoms up to $6 \sim 7 \mathrm{eV}$. The $532 \mathrm{~nm}$ second order harmonic output from another YAG laser pumps two dye lasers. The DCM red dye laser uses the same pumping scheme with the one in the tagging system. Another dye laser is tuned between $400 \mathrm{~nm}$ and $900 \mathrm{~nm}$. The two outputs are then used for four-wave mixing and produce laser radiation between $120 \mathrm{~nm}$ and $140 \mathrm{~nm}$. The second version directly uses the frequency tripled dye laser as the dissociation laser. The third version uses an excimer laser. The wavelength of the excimer laser can be $193 \mathrm{~nm}$ or $248 \mathrm{~nm}$. The excimer laser (about $200 \mathrm{~mJ}$ ) provides much more power than the dye laser $(2 \sim 3 \mathrm{~mJ})$, but the dye laser $(0.004 \mathrm{~nm})$ has much narrower bandwidth than excimer laser $(0.5 \mathrm{~nm})$.

\subsubsection{Experimental geometry and timing, and data acquisition}

Figure 3.14 shows the geometry of the experiments described in this thesis. Part of it has been shown schematically in sections 3.1 and 3.2. A rotationally cold hydrogen halide molecular beam is formed via supersonic expansion through a pulsed general valve. After passing skimmer 1, the molecular beam is crossed by the photodissociation laser. A small portion of the $\mathrm{H}$ atom photofragments fly through skimmer 2 in a direction perpendicular to the molecular beam and 
dissociation laser, pass two differential pumping stages (not shown in the figure), enter the UHV scattering chamber, and then collide with a single crystal surface. The surface is mounted on a 6axis manipulator, which can vary the polar $\left(\theta_{\mathrm{i}}\right)$ and azimuthal $\left(\varphi_{\mathrm{i}}\right)$ incidence angle. Here, the azimuthal angle is defined as the angle of the surface [10 $\overline{1}$ ] direction to the plane defined by incidence atom beam and surface normal. Before the scattering experiments are carried out, the surface is retracted upwards to a surface preparation chamber, which is not shown in the figure. In the surface preparation chamber, the standard cleaning and characterization procedures described in section 3.3 is carried out. At a short distance $(0.7 \mathrm{~mm})$ from the surface, the scattered $\mathrm{H}$ atoms are excited to the Rydberg state described in section 3.2. The tagged $\mathrm{H}$ atoms fly $255 \mathrm{~mm}$ and are then field ionized and detected. The detector is rotatable in the plane defined by the incidence atomic beam and the surface normal. A detector aperture $15 \mathrm{~cm}$ in front of the detector defines a $3^{\circ}$ angular resolution for the experiment. The scattering experiment is conducted under UHV $\left(3 \times 10^{-10} \mathrm{mbar}\right)$. Two differential pumping stages are used to separate the UHV chamber and the high vacuum supersonic expansion chamber, in which the pressure is about $10^{-5}$ mbar when the nozzle is running.

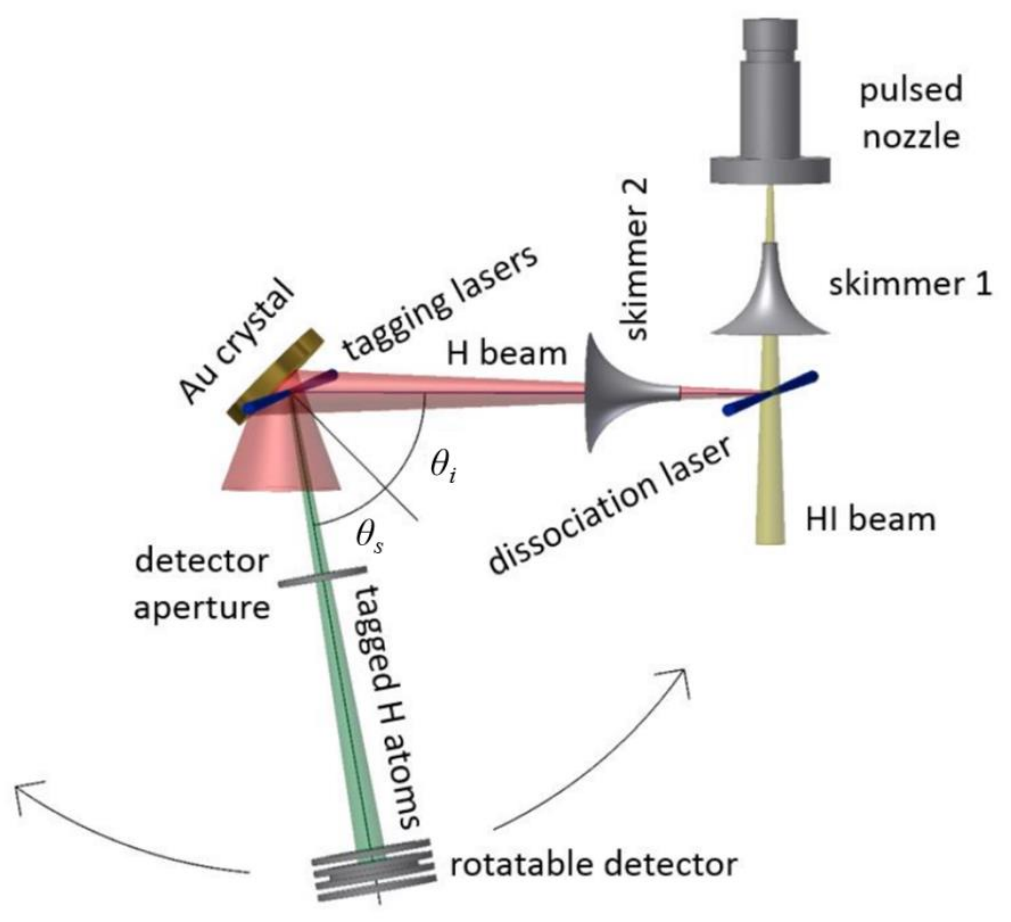

Figure 3.14: Geometry of the experimental setup. From Ref. (60). Reprinted with permission from $A A A S$. 
The detector is a Z stacked MCP assembly. The output current of the MCP is amplified by a fast 20x amplifier. The amplified signal can be directly measured as analogue signal by a Lecroy oscilloscope or passed through a discriminator and then measured as counting signal by a multichannel scaler. The experiments are conducted using $30 \mathrm{~Hz}$ nanosecond lasers (10 ns), so the timing must be carefully controlled. The timing of the experiment is controlled by a SRS DG635 delay generator. All the absolute times for different instruments are delayed to the reference time zero of the DG635. The delay between the pulsed nozzle opening and the firing of the dissociation laser is about $300 \mu \mathrm{s}$. The delay between the dissociation and tagging lasers varies between a few microseconds to several tens of microseconds depending on the incidence energy (7 14 $\mu$ s in experiments described in this thesis). 


\section{$4 \mathrm{H}$ and $\mathrm{D}$ atoms scattering from $\mathrm{Au}(111)$ and insulator}

As discussed in Chapter 1, to what extent of the hydrogen atom translational energy loss to metal surfaces at chemically interesting energies is adiabatic (phonons excitation) or non-adiabatic (ehps excitation) is still an open question (15). In this chapter, this question will be answered. Our approach is to compare the hydrogen atom translational energy loss between scattering from a $\mathrm{Au}(111)$ surface and from an insulating surface which is supposed to have mainly adiabatic excitations due to its band gap. Results from MD simulations with and without electronic excitations are used to compare to the experimental results (54). This chapter is organized as follows. Section 4.1 begins with experimentally obtained energy loss distributions for $\mathrm{H}$ atoms scattering from $\mathrm{Au}(111)$ and from Xe layer. Next, theoretical results from MD simulations neglecting and including electronic excitations are compared to the experimental data. In section 4.2, experimental data of $\mathrm{H}$ and $\mathrm{D}$ atoms scatterings from $\mathrm{Au}(111)$ are presented. Existing results from other studies are compared to the recent data.

\subsection{H atoms scattering from $\mathrm{Au}(111)$ and insulating Xe layer}

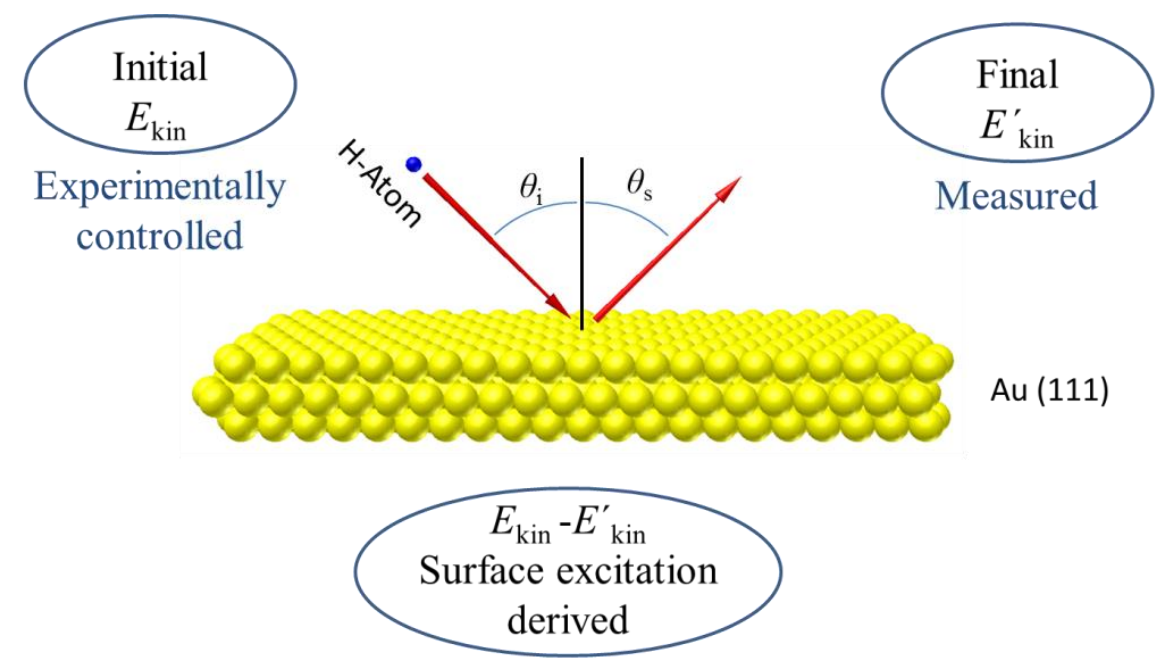

Figure 4.1: Sketch for the experiment of hydrogen atom scattering from Au(111) surface.

Figure 4.1 shows the sketch of the experiment. Hydrogen atoms are scattered from a single crystal $\mathrm{Au}$ (111) or from a thick Xe layer on $\mathrm{Au}(111) . \mathrm{Au}(111)$ is cleaned and characterized as described in Section 3.4. Insulating Xe layer is prepared by dosing the Au(111) with $\mathrm{Xe}$ gas at 
45K for 300 Langmuir, indicating that the Xe layer is thick enough to avoid the influence from the underlying $\mathrm{Au}(111)$. Scattering angle resolved translational energy loss distributions obtained via Rydberg atom tagging time-of-flight provide information about the surface excitations.

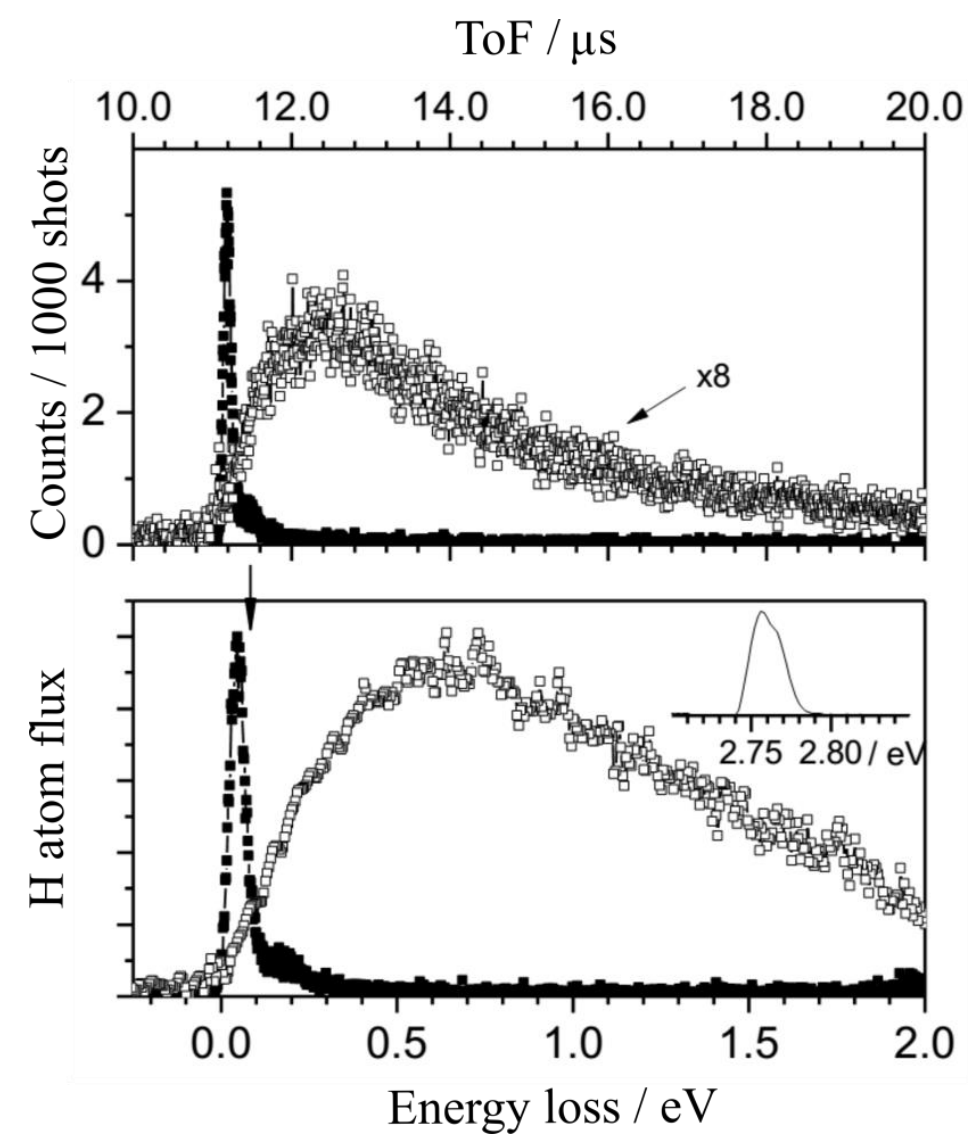

Figure 4.2: Time-of-flight (upper panel) and corresponding energy loss distribution of $H$ atoms scattering from Au(111) (empty black square) and Xe layer on Au(111) (solid black square). The black arrow indicates the energy loss for a binary collision between the $H$ atom and the Xe atom. The inset shows the translational energy distribution of the incidence atoms (at incidence energy $\left.E_{\mathrm{i}}=2.76 \mathrm{eV}\right)$. The incidence angle $\left(\theta_{i}\right)$ and scattering angle $\left(\theta_{s}\right)$ are both $45^{\circ}$. Incidence and scattered beams are in the plane defined by surface normal and [101] direction. From Ref. (60). Reprinted with permission from AAAS.

Figure 4.2 shows the results of $\mathrm{H}$ atom scattering from the clean single crystal $\mathrm{Au}(111)$ and the insulating Xe layer. Upper panel shows the time-of-flight spectra, and lower panel shows the corresponding energy loss spectra generated via using proper Jacobin. Empty black squares represent data from scattering on the $\mathrm{Au}(111)$, and solid black squares represent data from 
scattering on the Xe layer. Incidence energy of the beam is $2.76 \mathrm{eV}$, as shown in the inset of lower panel. The incidence and scattering angles are both $45^{\circ}$. Incidence and scattered beam are in the plane defined by the surface normal and [10 $\overline{1}]$ direction.

Obvious differences are observed between scattering on the $\mathrm{Au}(111)$ and on the insulating $\mathrm{Xe}$ layer. The energy loss for scattering on the Xe layer is small and exhibits a narrow distribution, indicating nearly elastic scattering. The most probable energy loss is $46 \mathrm{meV}$, smaller than the binary collision Baule limit of $83 \mathrm{meV}$ shown as the vertical arrow in Figure 4.2. The deviation may come from a larger effective mass of the Xe surface as discussed in Chapter 2. On the contrary, $\mathrm{H}$ atoms scattering from the $\mathrm{Au}(111)$ suffer larger energy loss and have a much broader distribution. The average energy loss is $910 \mathrm{meV}, 20$ times larger than the energy loss for scattering on the Xe solid. The broad distribution indicates that a broad continuum of accepter states in the solid contribute to the translational energy loss.

A binary electronically adiabatic collision model can capture the essence of the $\mathrm{H}$ atom scattering from the Xe solid, but the scattering on metal clearly needs more involved theory to describe. Two possible explanations for the large energy loss and wide distribution are multiple electronically adiabatic collisions or non-adiabatic ehps excitations. In order to quantify the role of the non-adiabatic ehps excitations in hydrogen atoms scattering from $\mathrm{Au}(111)$, MD simulations self-consistently including electronic excitations are conducted under the same incidence and scattering conditions as used in the experiment by Svenja Janke et al. (54). The MD simulations are carried out on a global full dimensional PES analytically fitted from DFT energy points via effective medium theory (EMT). EMT intrinsically contains the embedded electron densities which are used to self-consistently describe the non-adiabatic electronic excitations. Local density electronic friction approximation (LDFA) is used to describe the electronic excitations as a friction force felt by the atom during its moving. The friction force $f=-m \eta(\overrightarrow{\boldsymbol{r}}) \vec{v}$ is proportional to the velocity of the atom $\vec{v}$ and the electronic friction coefficient $\eta(\overrightarrow{\boldsymbol{r}})$ which depends only on the local electron density on the LDFA level. 


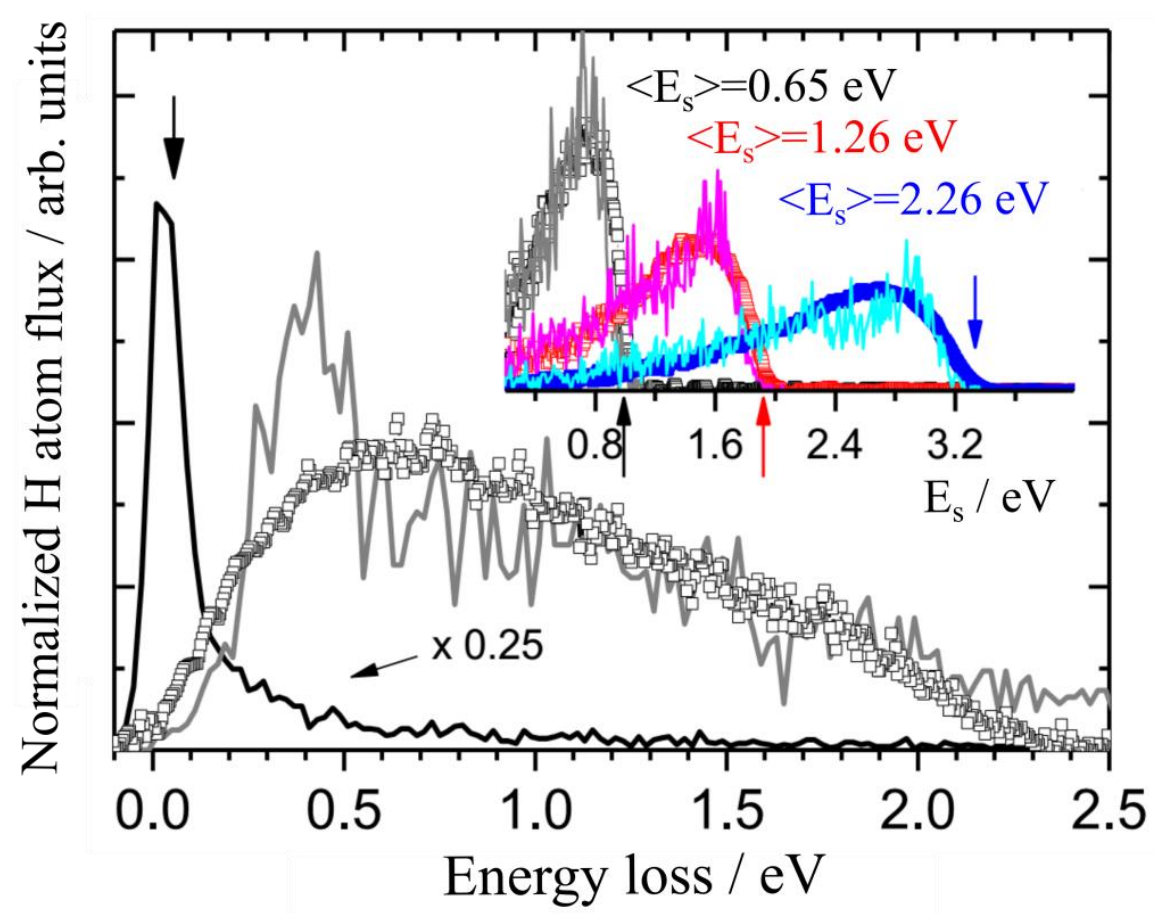

Figure 4.3: Comparison of experimentally obtained translational energy loss spectrum to theoretical simulations. Experimental energy loss distribution at incidence energy of $2.67 \mathrm{eV}$ is represented by empty squares. Calculated energy loss distributions with (grey) and without (black) electronic excitations are represented by solid lines. The vertical arrow marks the expected energy loss for a binary collision between an $\mathrm{H}$ and an Au atom. The inset shows the translational energy loss spectra at three different incidence energies. The blue (3.31 eV), red $(1.92 \mathrm{eV})$ and black $(0.99 \mathrm{eV})$ empty circles represent the experimentally obtained data. Colored arrows mark the three incidence energies. The cyan, magenta and gray solid lines represent the theoretically calculated data (with electronic excitations). The average energy losses for different incidence energies are shown as $\left\langle E_{s}\right\rangle$. The incidence and scattering angles are both $45^{\circ}$. The incidence and scattering beams are in the plane defined by the surface normal and [101] direction. From Ref. (60). Reprinted with permission from AAAS.

Figure 4.3 shows the comparison of experimentally obtained translational energy loss spectra to theoretical calculations. For the main panel, the incidence energy is $2.76 \mathrm{eV}$. The incidence and scattering angles are both $45^{\circ}$. The incidence and scattering beams are in the plane defined by the

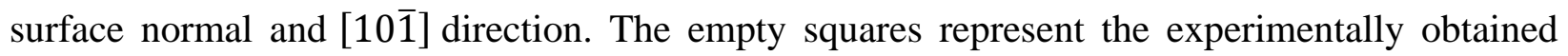
translational energy loss distribution. The solid lines represent the results from MD simulations 
neglecting (black) and including (gray) electronic excitations. It is obvious that calculation without electronic excitations totally fails to capture the observed magnitude of the $\mathrm{H}$ atom translational energy loss. In contrast, the simulation including the electronic excitations matches the experimental result quite well. The inset of Figure 4.3 shows the translational energy loss of $\mathrm{H}$ atom scattering from $\mathrm{Au}(111)$ for different incidence energies. For all incidence energies, agreement between experimental and theoretical results (with electronic excitations) is good. The fractional energy loss (the average energy loss normalized to the incidence energy) is equal to $0.33 \pm 0.01$ for all incidence energies, indicating that the non-adiabatic excitations remain important even at reduced energies.

Based on the results shown above, it is clear that the translational energy loss of $\mathrm{H}$ atom on metal surfaces is mainly non-adiabatic. Compared to the adiabatic energy loss of $\mathrm{H}$ atom on Xe layers, the non-adiabatic energy loss of $\mathrm{H}$ on $\mathrm{Au}(111)$ is large and shows broad distribution. The effective conversion of $\mathrm{H}$ atom translational energy to electronic excitations can be quantitatively confirmed by MD simulations including electronic excitations (electronic friction model on the level of LDFA).

\subsection{Isotope effect of hydrogen atoms scattering from $\mathrm{Au}(111)$}

In this section, the isotope effect of hydrogen atom scattering on $\mathrm{Au}(111)$ is discussed. As shown in section 4.1, we confirmed that $\mathrm{H}$ atom scattering on $\mathrm{Au}(111)$ is mainly non-adiabatic, and an electronic friction model based on LDFA could describe the non-adiabatic excitations. Nonadiabatic excitation is believed to exhibit strong isotope effect. Due to the proportionality of the electronic friction force to the velocity of the atom, $\mathrm{H}$ atom shows stronger non-adiabatic excitations than $\mathrm{D}$ atom does for the same kinetic energy. A strong isotope effect has been observed in the chemi-current measurements. Chemicurrents induced by $\mathrm{H}$ atom adsorption are typically 2 5 times larger than those induced by $\mathrm{D}$ atom adsorption $(18,21)$. This isotope effect is confirmed by another electronic friction based calculation (23).

However, things could be different in atom-surface scattering experiments. For the same incidence energy, the $\mathrm{H}$ atom exhibits stronger non-adiabatic excitations, but the $\mathrm{D}$ atom shows stronger adiabatic excitations. In order to get a quantitative answer for this problem, we studied the isotope effect in scattering experiments by exchanging $\mathrm{H}$ atom with $\mathrm{D}$ atom. The isotope 
effect is studied in two ways, same incidence energy and same incidence speed, considering the speed dependence of the electronic friction.

\subsubsection{Comparison of $H$ and $D$ atoms scattering from $A u(111)$}

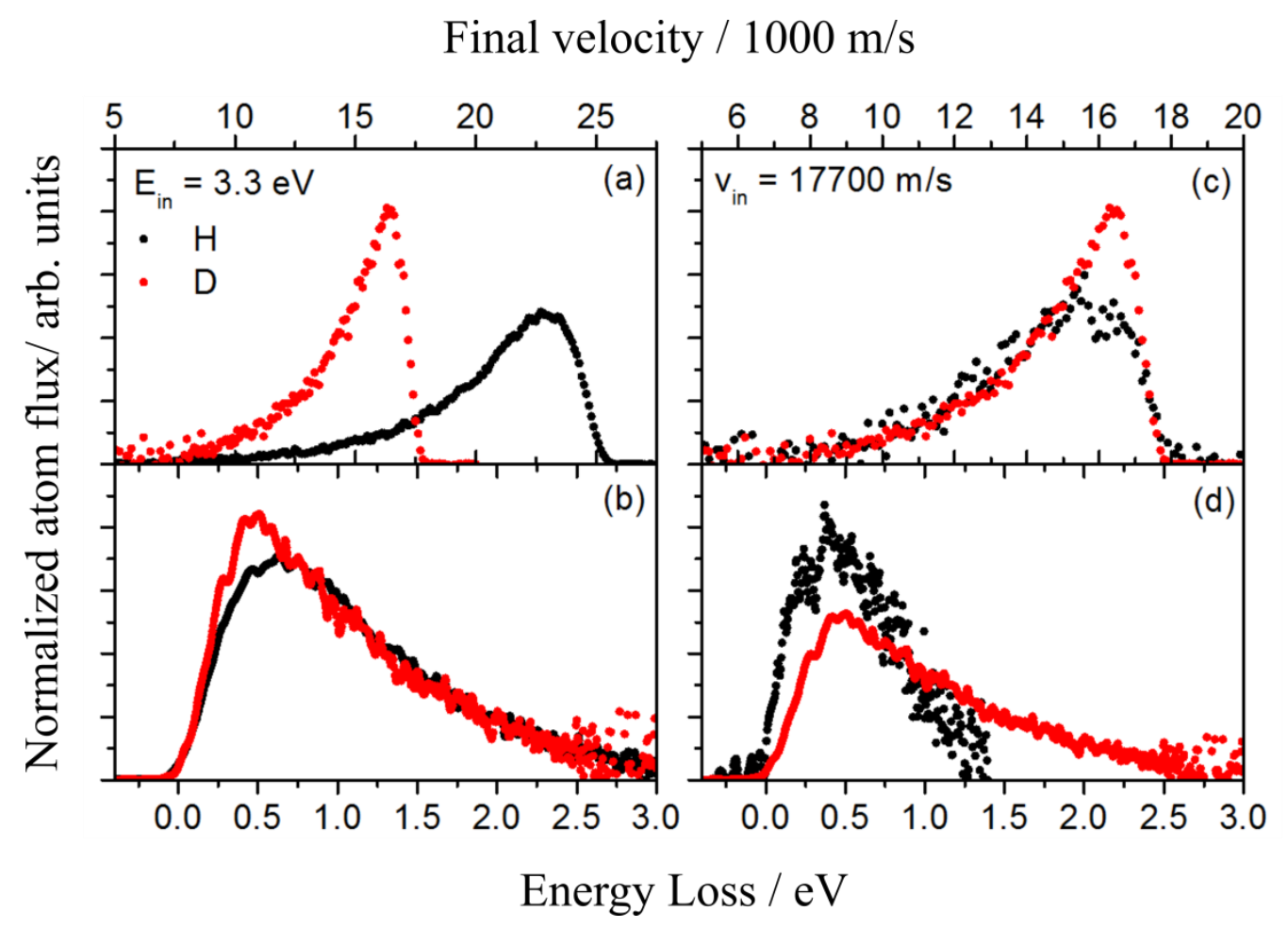

Figure 4.4: $H$ and $D$ atom scattering from Au(111). Black dots represent data from $H$ atom scattering, and red dots represent data from D atom scattering. Panel (a) and (b) show the speed and energy loss distribution for $H(3.31 \mathrm{eV})$ and $D(3.25 \mathrm{eV})$ atoms scattering at similar incidence energies. Panel $(c)$ and $(d)$ show the speed and energy loss distribution for $H$ and $D$ atoms scattering at the same incidence speed $(17.7 \mathrm{~km} / \mathrm{s})$. All distributions are normalized to the integral. The incidence and scattering angles are both $45^{\circ}$. The incidence and scattering beams

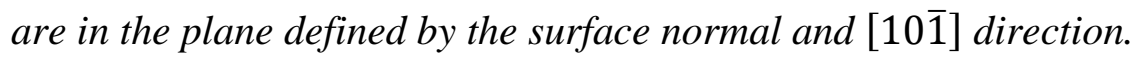

Panel (a) and (b) of Figure 4.4 show the speed and translational energy loss distributions for $\mathrm{H}$ (3.31 eV) and $\mathrm{D}(3.25 \mathrm{eV})$ atom scattering from $\mathrm{Au}(111)$ at similar incidence energies. The incidence and scattering angles are both $45^{\circ}$. The incidence and scattering beams are in the plane defined by the surface normal and [10ㅍ] direction. In terms of translational energy loss, only a small isotope effect is observed. The average energy losses are $1080 \mathrm{meV}$ for $\mathrm{H}$ and $960 \mathrm{meV}$ for 
$\mathrm{D}$, indicating an isotope effect of $\left\langle E_{\text {loss }}\right\rangle /\left\langle E_{\text {lossD }}\right\rangle=1.12$. The shapes of both translational energy loss distributions are similar. Only minor deviations are observed at small translational energy losses. Panel (c) and (d) show the speed and translational energy loss distribution for $\mathrm{H}$ and D atom scattering from $\mathrm{Au}(111)$ at the same incidence speed of $17.7 \mathrm{~km} / \mathrm{s}$. The incidence energies are $3.25 \mathrm{eV}$ for $\mathrm{D}$ and $1.64 \mathrm{eV}$ for $\mathrm{H}$. On average, D atoms lose $960 \mathrm{meV}$ and $\mathrm{H}$ atoms lose 540 $\mathrm{meV}$. However, the isotope effect on the speed distributions for $\mathrm{H}$ and $\mathrm{D}$ is minor. Deviations can only be seen at high speeds.

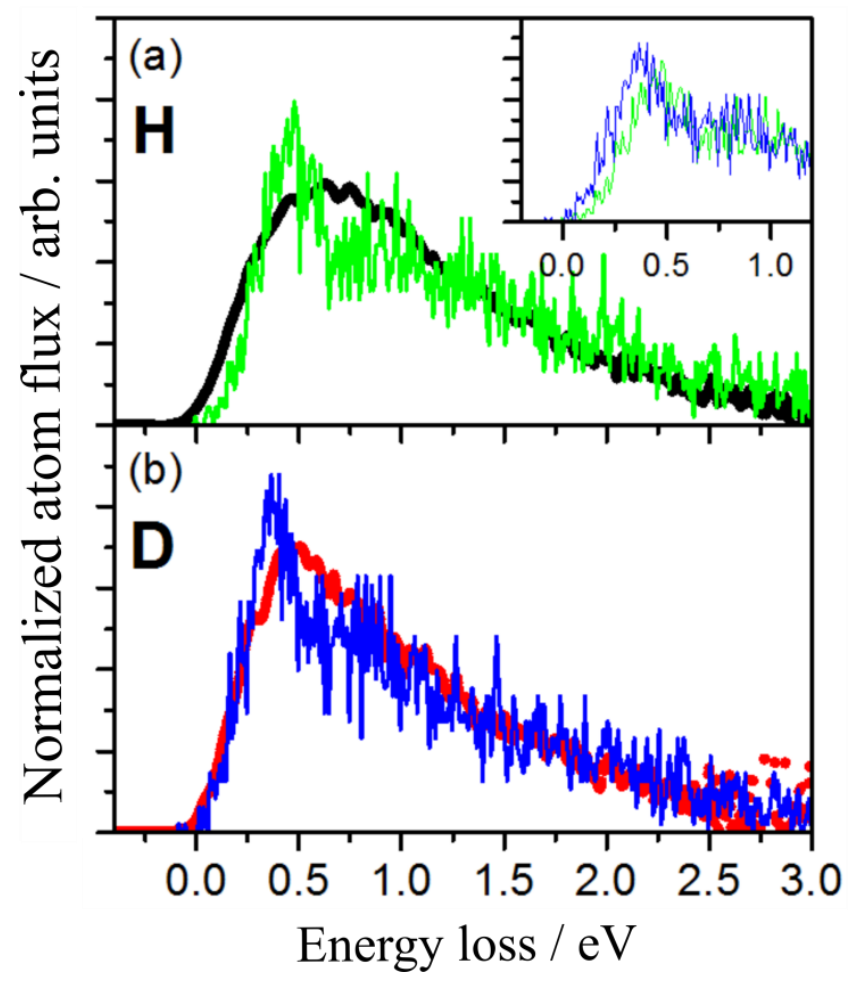

Figure 4.5: Comparisons between experimentally obtained and theoretically calculated energy loss distributions of $H$ and $D$ atoms scattering from $A u(111)$. The theoretical results are from MD simulations including electronic frictions (described in section 4.1). Panel (a) and (b) show the comparisons for $H$ and $D$, respectively. Black and red lines show the experimental data, and green and blue lines represent the theoretical results. The inset in panel (a) shows the comparison of theoretical results for $H$ (green) and $D$ (blue). The incidence and scattering conditions are the same as those shown in panel $(a)$ and $(b)$ of Figure 4.4.

The small isotope effect in the scattering experiments is stunning compared to chemi-current measurements. In order to understand the difference in the two experiments, MD calculations 
described in Section 4.1 were carried out to simulate the H and D atoms scatterings on $\mathrm{Au}(111)$ by Svenja Janke et al. Figure 4.5 shows the comparison between the experimental and calculated results. The incidence and scattering conditions are the same as those shown in Panel (a) and (b) of Figure 4.4. Experiment and theory agree well with each other for both $\mathrm{H}$ and D atoms scattering. For theoretical simulations, the calculated average translational energy loss is 1220 $\mathrm{meV}$ for $\mathrm{H}$ and $1080 \mathrm{meV}$ for $\mathrm{D}$, slightly overestimating the experimental values. The calculated isotope effect $\left\langle E_{\mathrm{lossH}}\right\rangle \mid\left\langle E_{\mathrm{loss}}\right\rangle=1.13$ is very close to the experimental value. The inset in Panel (a) shows a direct comparison of theoretical results for $\mathrm{H}$ and $\mathrm{D}$ atoms scattering. The deviation in the distributions at small energy losses is weaker than the experimental measurements but still presents in the model calculations.

\subsubsection{Discussion on the isotope effect of $H / D$ atoms scattering from $\mathrm{Au}(111)$}

Detailed analysis on calculated results gives insights on the scattering process. The small isotope effect in scattering experiment arises from the compensation of adiabatic phonon excitations and non-adiabatic ehps excitations. Owing to the higher mass, the efficiency of phonon excitations is higher for $\mathrm{D}$, but the efficiency of electronic excitations is decreased than $\mathrm{H}$ due to a lower speed at the same incidence energy. By separating the contributions of phonon and electronic excitations, the simulation predicts that $90 \%$ of the translational energy loss transfers to electronic system for $\mathrm{H}$, while it is $79 \%$ for $\mathrm{D}$.

The same incidence velocity scattering experiment provides us insights to understand the compensation effect. Under the framework of electronic friction theory, electronic excitations scales with speed only and should be the same for $H$ and D atoms at the same velocity. Assuming phonon excitations scale linearly with atomic mass and atom translational energy. At the same velocity, D atoms lose 4 times more energy to phonon excitations than $\mathrm{H}$ atoms $\left(\Delta E_{p, D}=4 \cdot \Delta E_{p, H}\right)$, but electronic excitations should be the same for $\mathrm{H}$ and $\mathrm{D}\left(\Delta E_{e, D}=\right.$ $\Delta E_{e, H}$ ). This is a good approximation for single bounce events (due to the small change in velocity during scattering). The difference between the observed energy losses is then only caused by the difference in phonon coupling caused by different masses. According to the unpublished theoretical data from Alexzander Kandratzenka et al, the average energy loss for single bounce is close to the most probable energy loss. In experiments, the most probable energy losses are $380 \mathrm{meV}$ for $\mathrm{H}$ and $500 \mathrm{meV}$ for $\mathrm{D}$, which results in electronic excitations 
$\Delta E_{e, D}=\Delta E_{e, H}=340 \mathrm{meV}$ and phonon excitations $\Delta E_{p, D}=4 \cdot \Delta E_{p, H}=160 \mathrm{meV}$. This means that $89 \%$ of the energy loss transfers to the electronic system for $\mathrm{H}$, and that $68 \%$ of the energy loss transfers to the electronic system for D. These experimentally deduced proportions are close to the theoretically predicted values ( $90 \%$ for $\mathrm{H}$ and $79 \%$ for $\mathrm{D})$.

\subsection{Summary}

In this chapter, I present the results of $\mathrm{H}$ atoms scattering on $\mathrm{Au}(111)$ and on Xe layer. $\mathrm{H}$ atoms scattering on Xe layer could be understood by a binary collision model. In contrast, $\mathrm{H}$ atoms scattering on $\mathrm{Au}(111)$ mainly excite ehps. $\mathrm{H}$ and $\mathrm{D}$ atoms scattering on $\mathrm{Au}(111)$ show minor isotope effect. The small isotope effect can be explained by the compensation between adiabatic phonon excitations proportional to the atomic mass and non-adiabatic ehps excitations proportional to the atom speed. The experimental findings can be quantitatively reproduced by MD simulations including non-adiabatic ehps excitations as electronic friction. 


\section{Hydrogen Atoms Interaction with Graphene: Background}

As described in chapter 4, energy transfer in scattering $\mathrm{H}$ atoms from metal surfaces is dominated by non-adiabatic electron-hole pair excitations, while adiabatic energy loss dominates in scattering $\mathrm{H}$ atoms from insulating surfaces. In order to obtain a complete picture of energy transfer in atoms scattering from surfaces, studies on semiconducting surfaces are necessary. Vibrational dynamics of $\mathrm{H}$ atoms adsorbed on semiconductors such as $\mathrm{Si}$ and Ge have been studied for a long time, mostly by surface sum frequency generation (SFG) techniques (61-63). Energy transfer between different $\mathrm{H}$-semiconductor vibrational modes and substrate phonons is primarily adiabatic. This is reasonable considering that the band gap for $\mathrm{Si}(1.1 \mathrm{eV})$ and $\mathrm{Ge}(0.67$ $\mathrm{eV})$ are much larger than the vibrational energy of $\mathrm{H}$-semiconductor bond $(0.2 \sim 0.3 \mathrm{eV})$. But what if the energy of the $\mathrm{H}$ atom is greater than the band gap of the semiconductor? Would nonadiabatic excitations take place? In the following chapters, I will present studies on $\mathrm{H}$ atom scattering with translational energies of several $\mathrm{eV}$ from the zero band gap graphene. If $\mathrm{H}$ scattering from graphene is still adiabatic, $\mathrm{H}$ atom scattering from any semiconductor will probably be adiabatic at incidence energies of several $\mathrm{eV}$.

Besides the motivation mentioned above, $\mathrm{H}$ atom adsorption on graphene has drawn attention in very diverse research fields over a long period of time. Since 1970s, hydrogen atom adsorption on graphene like materials was believed to play a key role on the $\mathrm{H}_{2}$ molecule formation in the interstellar media $(64,65)$. Hydrogen adsorption on graphene also has practical importance in hydrogen storage (9) and nuclear fusion technology (66). Recently, patterned adsorption of hydrogen atoms on graphene has become important for graphene-based electronic and spintronic devices (67-70).

\subsection{Introduction to graphene properties related to atom scattering}

Graphene is a single two-dimensional layer of carbon atoms bound in a hexagonal lattice structure. It is a basic building block of graphitic materials of all other dimensionalities. It can be wrapped into 0D fullerenes, rolled into 1D nanotube or stacked into 3D graphite. Graphene studies have a history of nearly 70 years. As early as 1947, Wallace worked out the electronic structure of graphene (71). A long time after that, graphene was theoretically researched as an 'academic' material. Since 1970, graphene layers formed on transition metal surfaces have been 
studied using low energy electron diffraction (LEED) (72), electron energy loss spectroscopy (EELS) (73) and scanning microscopies (74). But at that time, graphene was mostly an unwanted impurity in surface science research. The first isolation of a graphene monolayer was in 2004 and led to the Nobel Prize in 2010 (75).

Lattice structure. Figure 5.1 (a) shows the Bravais lattice structure of a graphene layer (76). The structure can be seen as a triangular lattice with a unit cell consisting of two atoms. $\mathrm{a}_{1}$ and $\mathrm{a}_{2}$ are the lattice vectors. The distance $\mathrm{b}$ between neighbor $\mathrm{C}$ atoms is $1.42 \AA$. This determines the absolute value of lattice vectors $\mathrm{a}_{0}$ as $2.465 \AA$. Figure 5.1 (b) is the corresponding reciprocal lattice, and $b_{1}$ and $b_{2}$ are the lattice vectors. The first Brillouin zone is also a hexagon. The center of the first Brillouin zone is called $\Gamma$ point. The corner points are denoted by $\mathrm{K}$ and named Dirac points. The middle points between Dirac points $\mathrm{K}$ are named $\mathrm{M}$ points.

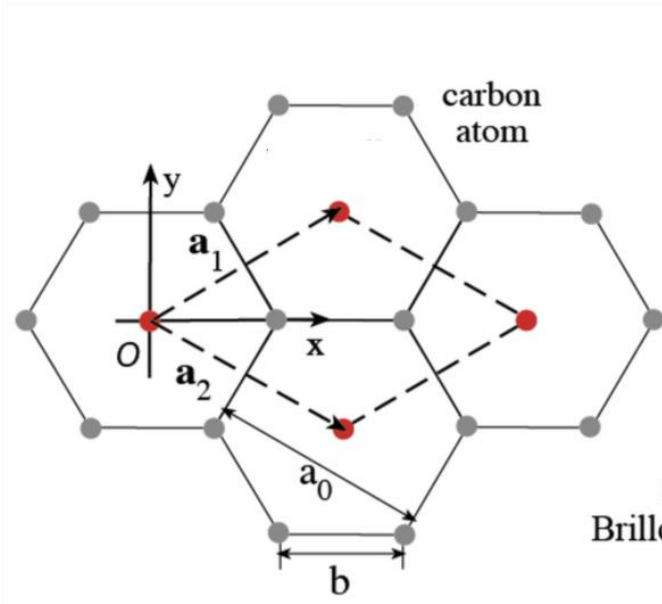

(a) Bravais lattice

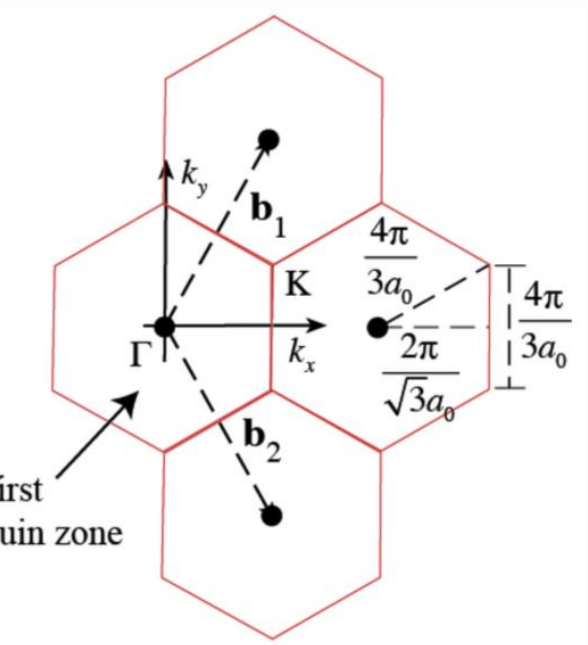

(b) Reciprocal lattice

Figure 5.1: Lattice structure of graphene. 


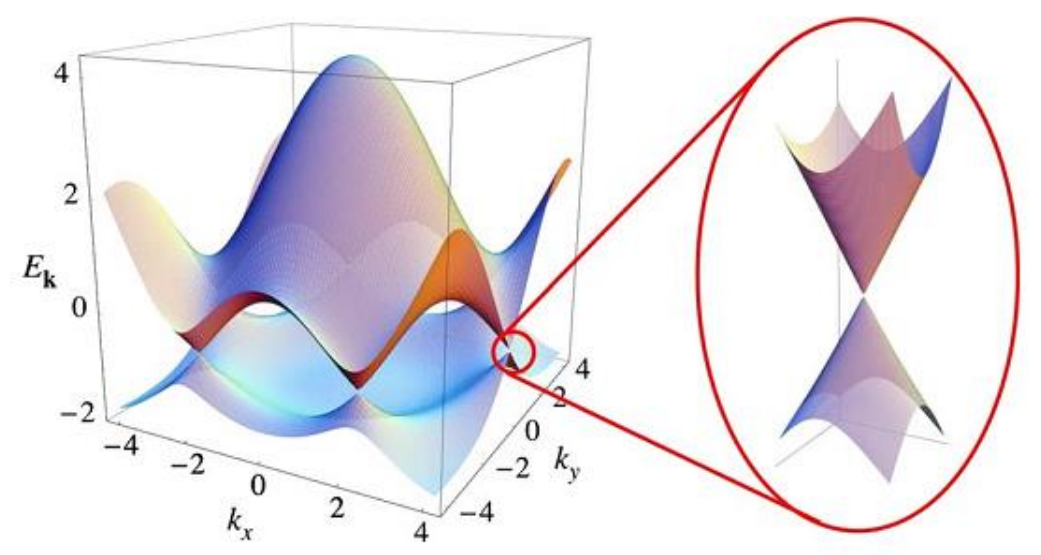

Figure 5.2: Left: Electronic dispersion of graphene. Right: enlarged view of the band structure close to one of the Dirac points. Reprinted figure with permission from Ref. (76). Copyright (2009) by the American Physical Society.

Electronic band structure. Figure 5.2 shows the band structure of graphene calculated from tight binding approximation (76). The upper and lower surface plots in the figure represent the conduction band and the valance band respectively. It can be seen in the figure that the conduction band and valence band meet at the Dirac points, leading to the zero band gap structure. The Fermi energy, also located at the Dirac points, is normally defined as the zero energy in the band structure. Near the Dirac points, the conduction band and the valence band are symmetric. Electrons whose energy is within about $1 \mathrm{eV}$ of the Dirac point have a linear dispersion relation. The dispersion relation at the Dirac points is expressed as:

$$
E_{ \pm}(\boldsymbol{k}) \approx \pm \hbar v_{F}|\boldsymbol{k}-\boldsymbol{K}|
$$

$\boldsymbol{k}$ is the wave vector of the carrier. $\boldsymbol{K}$ is the wave vector for the Dirac point. $v_{F} \approx 1 \times 10^{6} \mathrm{~m} / \mathrm{s}$ is the Fermi velocity. $E_{ \pm}(\boldsymbol{k})$ denote energies of the conduction band and valence band respectively. The distinctive band structure is the primary reason for the unusual electronic properties of graphene. The linear dispersion relation shown in Eq. 5.1 resembles that of light. This means the charge carriers are analogues to relativistic particles and more easily and naturally described by the Dirac equation rather than by the Schrödinger equation. It provides a way to probe quantum electrodynamics phenomena by measuring graphene's electronic properties (77). Graphene 
displays remarkable electron mobility at room temperature, leading to a resistivity of $10^{-6} \Omega \cdot \mathrm{cm}$, which is even lower than the resistivity of silver (78).

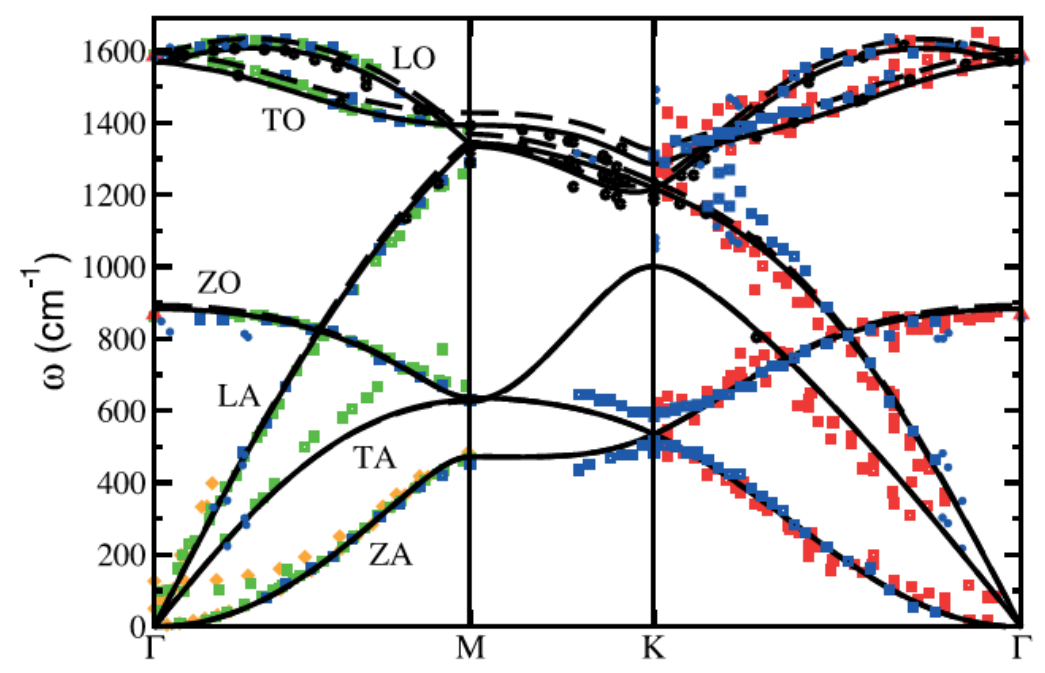

Figure 5.3: Phonon dispersion relation in graphite following surface cuts along lines $\Gamma$ to $M, M$ to $K$, and $K$ to $\Gamma$ in the first Brillouin zone. Black solid curves: results from DFT calculation within generalized gradient approximation (GGA). Black dash curves: results from DFT calculation within local density approximation (LDA). Yellow diamonds: data from neutron scattering. Green squares: data from electron energy loss spectrum (EELS). Blue squares: data from electron energy loss spectrum. Red squares: data from electron energy loss spectrum. Black circles: data from X-ray scattering. Blue circles: data from double resonance Raman scattering. Red triangles: data from IR absorption. Reprinted from Ref. (79). Copyright (2004) with permission from Elsevier.

Phonon dispersion. Graphene is the second strongest material ever known. Its superlative status was recently replaced by another carbon allotrope carbine recently (80). Young's modules as high as $1 \mathrm{TPa}$ have been measured on graphene (81). Graphene also has the highest thermal conductivity. These characteristics are all related to the unique phonon dispersion relation of graphene. Figure 5.3 shows theoretically calculated and experimentally measured phonon dispersion relation in graphite. It is expected to be very similar to graphene. ZA and ZO represent the out-of-plane acoustic and optical modes, respectively. LA and LO represent the inplane longitudinal acoustic and optical mode. TA and TO are the in-plane sheer acoustic and optical modes. The lines are theoretical dispersion relations from DFT calculations. The dots 
represent experimental data obtained by various methods. The experimental measurements agree well with the theoretical calculation with the exception of the TA mode measurement along the $\Gamma$ to $\mathrm{M}$ direction. This is because the crystal contains different rotational domains (79).

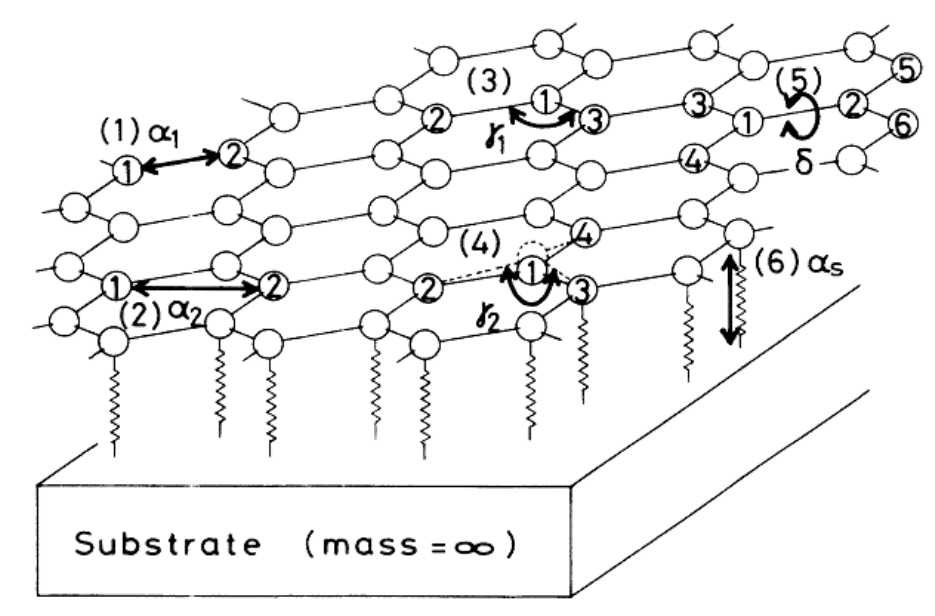

Figure 5.4: Illustration for the six force constant parameters. $\alpha_{1}$ and $\alpha_{2}$ are stretching force constants. $\gamma_{1}$ and $\gamma_{2}$ are bending force constant. $\delta$ is the twisting force constant and $\alpha_{s}$ represents the interaction between the substrate and the graphene. Reprinted with permission from Ref. (73). Copyright (1990) by the American Physical Society.

Besides $a b$ initio calculations, a semi-empirical potential model proposed by T. Aizawa $e t$ al. is often used to calculate the phonon dispersion of graphene layers on different substrate (73). Six phenomenological force constants (FC) are used to describe graphene. As shown in Figure 5.4, $\alpha_{1}$ is the stretching FC between two nearest neighbor atoms. $\alpha_{2}$ is the stretching FC between the second nearest neighbors. $\gamma_{1}$ represents a three body in-plane bending FC. $\gamma_{2}$ represents a four body out-of-plane bending FC. $\delta$ is the twisting FC, which is similar with a force constant keeping the ethylene molecule flat. $\alpha_{\mathrm{s}}$ is a stretching FC between the graphene and the substrate. The corresponding potential energy terms for the six force constants are:

$$
\begin{gathered}
\frac{\alpha}{2}\left[\frac{\left(\boldsymbol{u}_{2}-\boldsymbol{u}_{1}\right) \cdot \boldsymbol{r}_{12}}{\left|\boldsymbol{r}_{12}\right|}\right]^{2} \\
\frac{\gamma_{1}}{2}\left[\left[\frac{\left(\boldsymbol{u}_{2}-\boldsymbol{u}_{1}\right) \times \boldsymbol{r}_{12}}{\left|\boldsymbol{r}_{12}\right|^{2}}\right]_{z}-\left[\frac{\left(\boldsymbol{u}_{3}-\boldsymbol{u}_{1}\right) \times \boldsymbol{r}_{13}}{\left|\boldsymbol{r}_{13}\right|^{2}}\right]_{z}\right]^{2}
\end{gathered}
$$




$$
\begin{gathered}
\frac{\gamma_{2}}{2}\left[\frac{\left(u_{2 z}+u_{3 z}+u_{4 z}-3 u_{1 z}\right)}{\left|\boldsymbol{r}_{12}\right|}\right]^{2} \\
\frac{\delta}{2}\left[\frac{\left(u_{5 z}-u_{6 z}\right)-\left(u_{3 z}-u_{4 z}\right)}{a_{0}}\right]^{2} \\
\frac{\alpha_{s}}{2} u_{z}^{2}
\end{gathered}
$$

$\boldsymbol{u}_{i}$ denotes the displacement vector of atom $i$. Vector $\boldsymbol{r}_{i j}$ indicates the relative mean position of atom $i$ and atom $j . a_{0}$ is the lattice constant. Eq. 5.1 is the stretching energy term for both $\alpha_{1}$ and $\alpha_{2}$. The lower case $\mathrm{z}$ in Eq. 5.2 and 5.3 indicates the vector component perpendicular to the graphene surface. Dispersion relation can be calculated based on these potential energy terms. The six FCs are generated by fitting experimental data to the model.
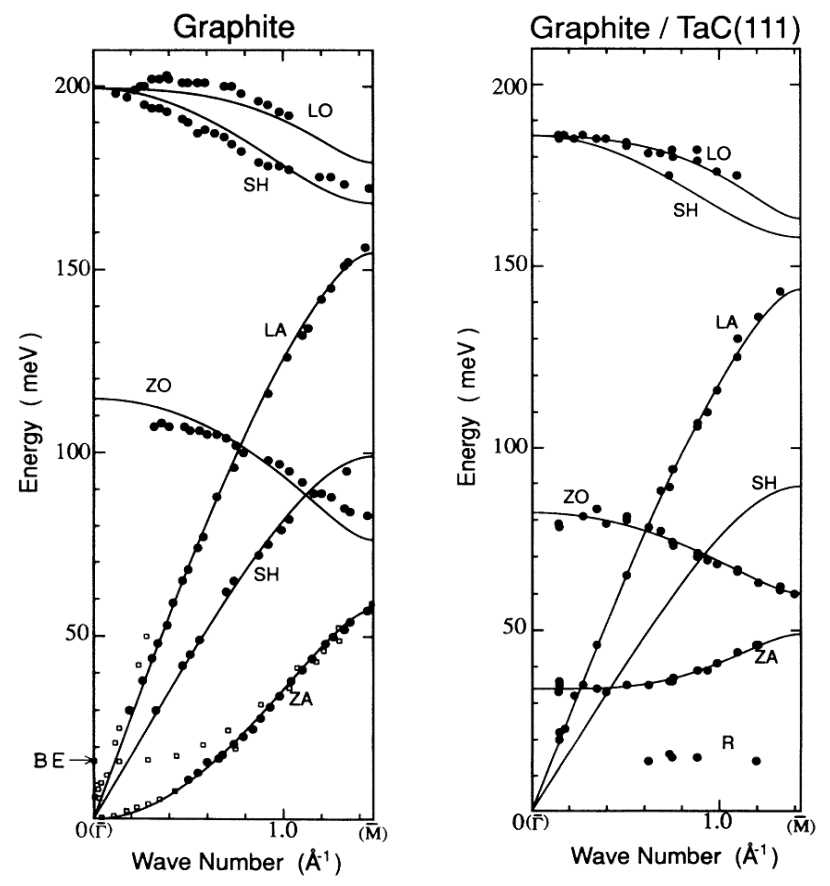

Figure 5.5: Phonon dispersion for bulk graphite and graphene on TaC(111). The black dots are results from experimental measurements. The black curves are results from 6 FCs model calculation fitted to the data. Hollow squares in the left panel are data from neutron scattering. In plane transverse modes are indicated by $S H$. The $R$ branch in the right panel is due to the substrate. Reprinted with permission from Ref. (73). Copyright (2009) by the American Physical Society. 


\subsection{Graphene fabrication and characterization}

Since graphene was first isolated by Novoselov and Geim using the Scotch tape method in 2004 (75), many methods have been developed to produce graphene. The major concern in graphene fabrication is producing samples on a large scale and low density of defects. In this section, I will introduce commonly used graphene fabrication and characterization methods.

\subsubsection{Brief introduction on graphene fabrication methods}

Mechanical exfoliation. Graphene is essentially the (0001) basal plane of graphite. Novoselov and Geim employed Scotch tape to repeatedly peel flakes off graphite and then transferred the flakes onto the surface of $\mathrm{SiO}_{2}$ wafer. Graphene of a few layers was first identified on the wafer using the contrast difference in an optical microscope, and then single layer graphene was picked out using SEM (82). The Scotch tape exfoliating method has been shown to produce high quality graphene up to $100 \mu \mathrm{m}$ in size. Even though this technique looks no more sophisticated than drawing with a pencil, there is no other method that can match mechanical exfoliation for producing high quality, high charge carrier mobility graphene. However, mechanical exfoliation is a time consuming process limited to small scale production. Instead of cleaving graphite manually, it is also possible to automate the process by using ultrasonic cleavage. Atomic planes in graphite can also be partially detached by intercalation before sonification. The sonification method allows graphene production on an industrial level (82).

Epitaxial growth. An alternative route is to grow graphene epitaxially on top of various substrates. Popular choices of substrates are transition metals (83-89) and carbides (90). Graphene is produced by heating $\mathrm{SiC}$ in UHV to temperatures between $1000{ }^{\circ} \mathrm{C}$ and $1500{ }^{\circ} \mathrm{C}$. High temperature causes $\mathrm{Si}$ atoms to sublimate from the material surface and leaves behind a graphene surface $(91,92)$. Similar processes are the segregation of body carbon in the transition metal to the surface. Dosing transition metals, for instance $\mathrm{Ni}$ and $\mathrm{Pt}$, with hydrocarbons at elevated temperatures increases density of the carbon atom in the bulk. A Graphene sample is formed on the surface during the subsequent cooling by carbon atom surface segregation $(85,89)$. These methods can produce large scale graphene but have a common disadvantage of multilayer growth. Single layer graphene sheets grown by these methods always contain some small multilayer regions. 
In contrast, epitaxial growth of graphene on transition metal surfaces via hydrocarbon dissociation can self-terminate once a monolayer is formed. Hydrocarbon molecules are dosed at elevated temperatures onto the transition metal surfaces, which are used as both catalyst and substrate to grow the graphene layer. Various metals have been used as substrates, for instance $\mathrm{Al}, \mathrm{Cu}, \mathrm{Ni}, \mathrm{Ir}, \mathrm{Ru}, \mathrm{Au}$ and Pt (83-89). Transition metal carbides can also be used as substrate. Large area graphene samples of the order of centimeters have been grown on copper foils (87). These methods make the large-scale production of graphene possible.

Even though epitaxial graphene can be synthesized to large scale, it does not typically have as good quality as mechanically exfoliated graphene. Properties of epitaxial graphene strongly depend on the growth conditions and substrates. A large size epitaxial graphene sample is not single crystalline but an integral of many domains (84). Varying the growth speed by substrate temperature and dosing flux controls the domain size and roughness of graphene layer (84). Step and defect densities of the substrates also limit the domain size. Due to the lattice mismatch, single layer graphene samples on $\mathrm{Au}(111)$ and $\mathrm{Pt}(111)$ have different domain rotations, while those on $\mathrm{Ni}(111)$ and $\mathrm{Ru}(111)$ are better aligned to the substrate $(93,94)$. The interactions of graphene and substrates are quite different. Epitaxial graphene is physisorbed on $\mathrm{Al}, \mathrm{Au}, \mathrm{Ag}, \mathrm{Cu}$ and $\mathrm{Pt}$, while chemisorbed on $\mathrm{Ni}, \mathrm{Co}, \mathrm{Pd}$ and $\mathrm{SiC}$ (93-96). Quality and property control of epitaxial graphene is currently still a developing field.

\subsubsection{Synthesis and characterization of epitaxial graphene on $\operatorname{Pt}(111)$}

We use epitaxial graphene grown on a $\mathrm{Pt}(111)$ surface for the experiments described in this work. The instruments used for the surface preparation and characterization are described in chapter 3 . The $\mathrm{Pt}(111)$ substrate is cleaned by repeated cycles of $\mathrm{Ar}^{+}$ion sputtering (30 mins) followed by annealing at $900{ }^{\circ} \mathrm{C}$ (30 mins) and then flashing to $1000{ }^{\circ} \mathrm{C}$ ( 2 mins, to ensure the cleanliness of the surface). Surface cleanliness and order are checked by Auger electron spectroscopy (AES) and low energy electron diffraction (LEED). If $\mathrm{Ar}^{+}$ion sputtering and annealing at $900{ }^{\circ} \mathrm{C}$ is not enough to remove carbon impurities on the $\mathrm{Pt}(111)$ surface, annealing at $650{ }^{\circ} \mathrm{C}$ in $\mathrm{O}_{2}$ atmosphere $\left(1 \times 10^{-7}\right.$ mbar $/ 30$ mins $)$ is used in between the $\mathrm{Ar}^{+}$ion sputtering and the $900{ }^{\circ} \mathrm{C}$ annealing. After the cleaning, ethylene $\left(\mathrm{C}_{2} \mathrm{H}_{4}\right)$ is dosed onto the clean $\mathrm{Pt}(111)$ substrate at $700{ }^{\circ} \mathrm{C}$. Single layer graphene is formed upon an exposure of $3 \times 10^{-8}$ mbar $\mathrm{C}_{2} \mathrm{H}_{4}$ for 15- 20 mins (25- 35 Langmuir). After removing the $\mathrm{C}_{2} \mathrm{H}_{4}$ from the chamber, the sample is held at $700{ }^{\circ} \mathrm{C}$ for another 
5 mins to pump all $\mathrm{C}_{2} \mathrm{H}_{4}$ gas out. The quality of the single layer graphene is confirmed by the in situ LEED and AES, and also by the comparison between scattering results from pristine Pt(111) surface and the graphene sample. The comparison will be shown in the next chapter.
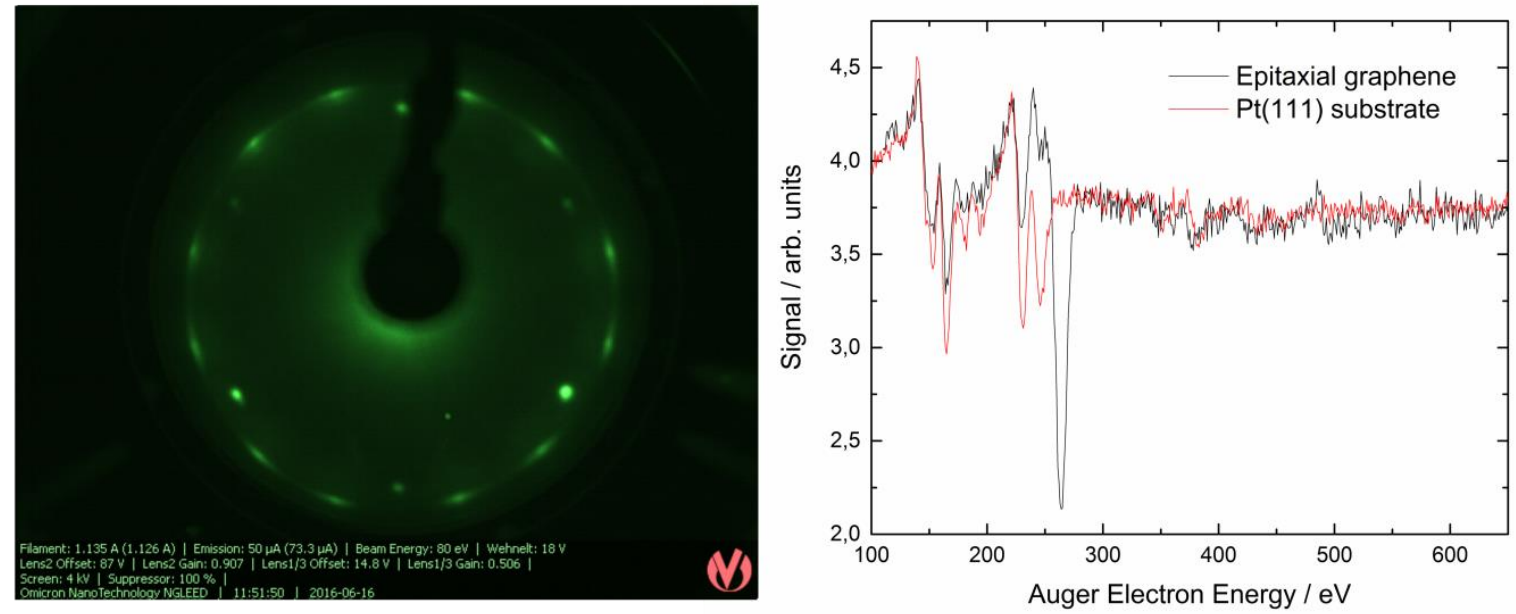

Figure 5.6: Left panel: measured LEED spectrum for epitaxial graphene on Pt(111). The electron beam energy is $80 \mathrm{eV}$. Right panel: comparison of AES between epitaxial graphene and Pt(111) substrate.

The left panel of Figure 5.6 shows the LEED pattern of epitaxial graphene on $\operatorname{Pt}(111)$. The electron energy used for the LEED measurement is $80 \mathrm{eV}$. The observed pattern is the first order diffraction pattern. The inner bright spots are from the electron diffraction on the $\operatorname{Pt}(111)$ substrate. The outer arc-like spots are from the electron diffraction on epitaxial graphene, and indicate the existence of different rotational domains. The arc-like spots instead of bright spots indicate weak interaction of epitaxial graphene and $\mathrm{Pt}(111)$ substrate. It is not strong enough to form a constant rotation angle between the epitaxial graphene and the $\operatorname{Pt}(111)$ substrate. The right panel of Figure 5.6 shows the comparison of AES between the clean $\operatorname{Pt}(111)$ and epitaxial graphene on $\mathrm{Pt}(111)$. The black curve is the AES of clean $\mathrm{Pt}(111)$ and shows the cleanliness of the Pt substrate. The red curve is the AES after the growth of graphene. The intensity of the Pt peak decreases due to the top most graphene layer, and there is only one new peak at $272 \mathrm{eV}$, which is due to $\mathrm{C}$. This ensures the cleanliness of the graphene sample. 


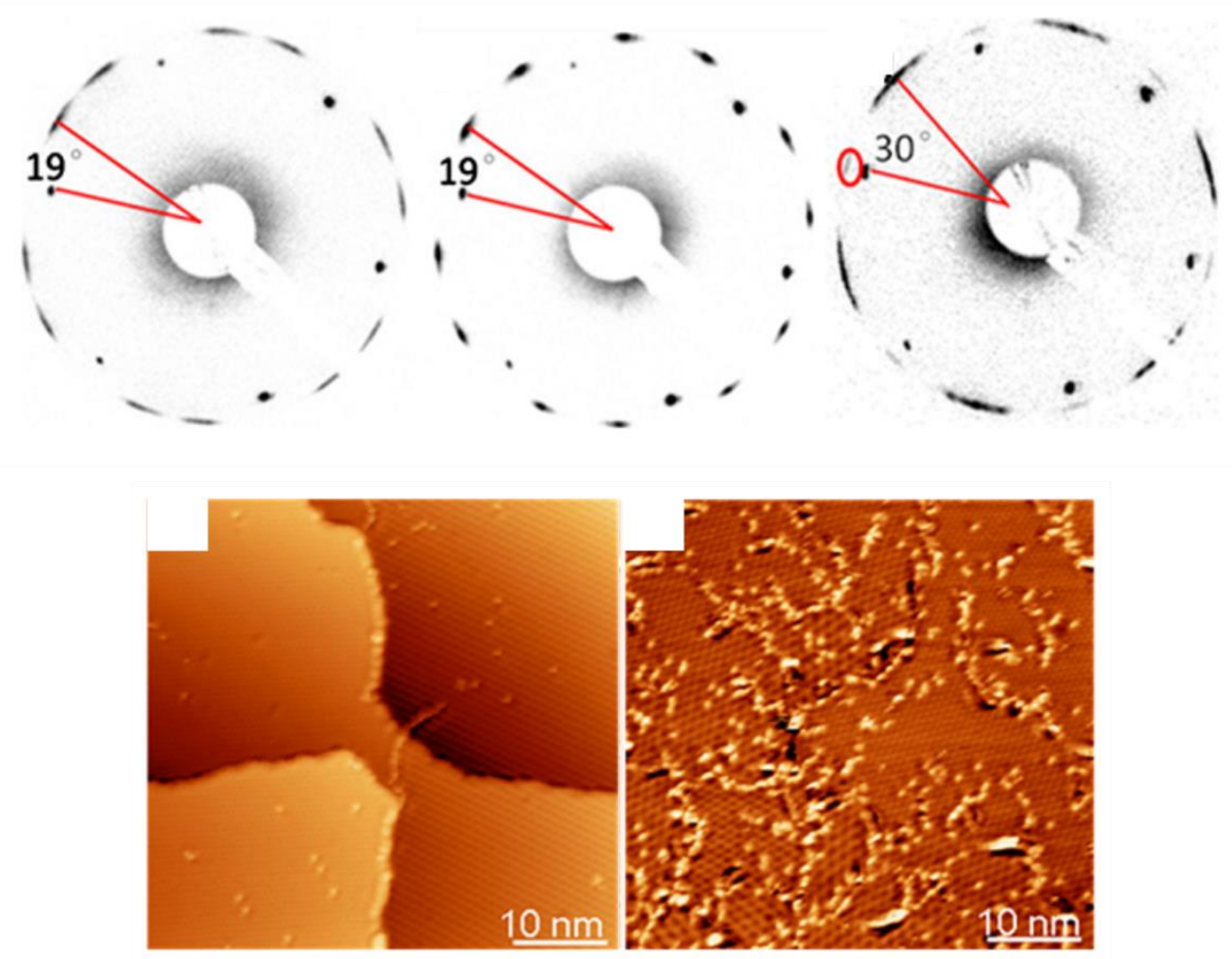

Figure 5.7: Upper panel: LEED pattern of epitaxial graphene on Pt(111) measured at different growth temperature (from left to right $773 \mathrm{~K}, 873 \mathrm{~K}$, and $973 \mathrm{~K})$ but same exposure $\left(5 \times 10^{-7} \mathrm{mbar}\right.$ / 37.6L). The beam energy is $60 \mathrm{eV}$. Lower panel: STM image of epitaxial graphene on Pt(111) $(\mathrm{V}=0.04 \mathrm{~V}, \mathrm{I}=1.5 \mathrm{nA})$ at different growth temperatures (from left to right $773 \mathrm{~K}$ and $1073 \mathrm{~K}$ ) and exposure (from left to right $5 \times 10^{-7} \mathrm{mbar} / 37.6 \mathrm{~L}$ and $5 \times 10^{-6} \mathrm{mbar} / 376 \mathrm{~L}$ ). The graphene was grown by exposing $\mathrm{Pt}(111)$ to $\mathrm{C}_{2} \mathrm{H}_{4}$ at high temperature. The growth conditions are the same for the upper left LEED pattern and lower left STM image. All measurement was done by M. Gao et al. Reprinted from Ref. (84), with the permission of AIP Publishing.

The characteristics of the epitaxial graphene related to atom-surface scattering include sample flatness, domain size, interaction with substrate, defect density, electronic structure, and phonon structure. Characterization of these properties requires various experimental methods such as scanning tunneling microscopy (STM), X-ray photoelectron spectroscopy (XPS) and angular resolved photoelectron spectroscopy (ARPES). The recipe we used to synthesis graphene is a well-studied method. M. Gao et al. correlated the sample flatness, domain size and electronic structure to the LEED pattern (84). T. Aizawa et al. and A. Politano et al. both compared the 
phonon dispersion of epitaxial graphene with graphite $(90,97)$. Several studies have been done on the interaction of graphene and $\operatorname{Pt}(111)$ substrate $(89,95,96)$. In the following paragraphs, I will give an overview of the points mentioned above.

Domain size and defect density: Figure 5.7 shows the LEED and STM measurements of epitaxial graphene grown by dosing $\operatorname{Pt}(111)$ substrate with $\mathrm{C}_{2} \mathrm{H}_{4}$ at elevated temperatures. The measurement was done by $\mathrm{M}$. Gao et al (84). The LEED patterns are from the electron diffraction on graphene grown at different temperatures (from left to right $773 \mathrm{~K}, 873 \mathrm{~K}$, and $973 \mathrm{~K})$ but the same exposure $\left(5 \times 10^{-7} \mathrm{mbar} / 37.6 \mathrm{~L}\right)$. For the growth temperature of $773 \mathrm{~K}$, the LEED pattern consists of diffuse arc-shape spots and has a preferential orientation of $19^{\circ}$ relative to the sharp $\operatorname{Pt}(111)$ diffraction spots. As the temperature is increased to $873 \mathrm{~K}$, the arc spots become narrower and brighter, indicating an increase of $\mathrm{R} 19^{\circ}$ domains. When the temperature is increased to $973 \mathrm{~K}$, the preferential orientation changes to $30^{\circ}$ relative to the $\mathrm{Pt}(111)$ diffraction spots, and faint spots in line with the Pt spots appear. Arc-shape instead of circularly-shaped spots are observed from LEED pattern of the graphene layer, indicating that the graphene is locally ordered but with varying orientation in different domains. The proportion of different rotational domains depends on the growth temperature. The formation of various graphene structures on $\operatorname{Pt}(111)$ at different temperatures is mainly attributed to the modulated energy barriers for attaching carbon atoms to the different orientation of graphene edges during the epitaxial growth process. The domain size and defect density is mainly determined by the nucleation rate, which can be controlled by the growth temperature. In the STM image, it is clearly seen that the low growth temperature and low exposure render large domain size and low defect density. Domains as large as several tens of nanometer are observed at $773 \mathrm{~K}$ growth temperature. The growth conditions for the lower-left STM image and upper-left LEED in Figure 5.7 are the same. Since the LEED pattern, domain size and defect density all depend on temperature, which is an indication of nucleation rate, the LEED pattern is used as an indication of domain size and defect density of the sample. By comparison of the LEED pattern of our sample with those in Figure 5.7, we deduce that our graphene layer should have low defect density and a domain size as large as $10 \mathrm{~nm}$. 

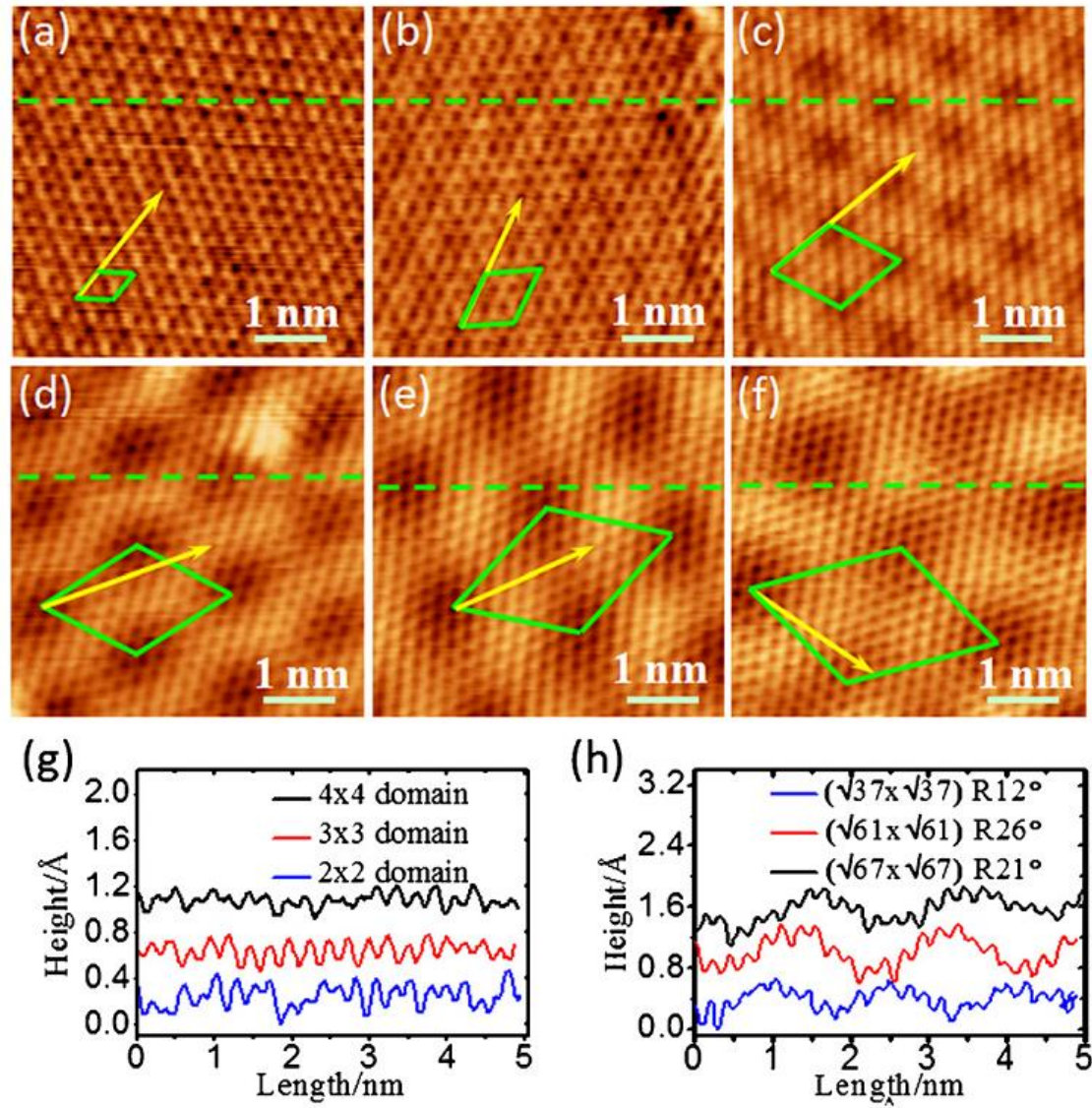

Figure 5.8: Upper panel $(a)-(f)$ : STM images of epitaxial graphene on Pt(111) with different Moiré structures. The green cells indicate the supercell of the Moiré periodicity. The yellow arrows indicate the lattice vector of graphene. Lower panel $(g)-(h)$ : Height profile measured along the green dash lines in $(a)-(f)$. Reprinted from Ref. (84), with the permission of AIP Publishing.

Graphene flatness: Different LEED patterns correspond to different Moiré structures, whose orientation and periodicity vary with the rotation angle between the graphene and underlying $\operatorname{Pt}(111)$ lattice in each domain. The upper panel (a) - (f) of Figure 5.8 shows the Moiré structures formed by graphene on $\operatorname{Pt}(111)$. The lattice vectors of Moiré structures shown in upper panel (a) - (c) are parallel to the lattice vector of graphene. The Moiré structures form $2 \times 2,3 \times 3$ and $4 \times 4$ unit cells, respectively. Their height profiles across the dashed green line in each STM images are shown in lower panel $\mathrm{g}$. The corrugations of these domains are within $0.03 \mathrm{~nm}$, indicating a flat graphene film without much distortion in the vertical direction. The lattice vectors of Moiré structures shown in upper panel (d) - (f) rotate small angles relative to the lattice vector of 
graphene. The Moiré structures have $(\sqrt{37} \times \sqrt{37}) \mathrm{R} 21^{\circ},(\sqrt{61} \times \sqrt{61}) \mathrm{R} 26^{\circ}$ and $(\sqrt{67} \times \sqrt{67})$ $\mathrm{R} 12^{\circ}$ unit cell, respectively. The corrugation of these domains increases to $0.05-0.08 \mathrm{~nm}$, as is shown in lower panel $\mathrm{h}$. The height difference originates from the different rotation angles of graphene relative to the $\operatorname{Pt}(111)$ substrate, which are $30^{\circ}, 19^{\circ}, 14^{\circ}, 6^{\circ}, 3^{\circ}$, and $2^{\circ}$ for Moiré structures shown in panel (a) - (f) in Figure 5.8. According to the LEED pattern we measured, our graphene layer has preferential rotation angle around $15^{\circ}$ relative to the $\operatorname{Pt}(111)$ substrate. The Moiré structure of our sample should be similar to the structure shown in upper panel (c) of Figure 5.8, indicating small corrugation of the epitaxial graphene.

Phonon dispersion: Figure 5.9 shows a comparison of the phonon dispersion of graphene on $\operatorname{Pt}(111)$ with that of graphite. The epitaxial graphene used in this research shows similar LEED pattern with ours shown in Figure 5.6, indicating similar graphene properties in the two studies. It is clearly seen in the figure that the phonon dispersions of graphene on $\operatorname{Pt}(111)$ and graphite are similar to one another, indicating that the underlying $\operatorname{Pt}(111)$ substrate does not significantly affect the phonon property of the epitaxial graphene (90). This is also seen in the elastic property measurement of epitaxial and pristine graphene (97). The Young's modules for pristine graphene and epitaxial graphene on $\operatorname{Pt}(111)$ are nearly the same. The weak influence of $\operatorname{Pt}(111)$ substrate on graphene phonon property is due to the weak interaction of $\operatorname{Pt}(111)$ and the graphene layer. For different substrates, the phonon dispersion of epitaxial graphene behaves differently, depending on the interaction with the substrate. Graphene on metals such as $\mathrm{Ni}$, where the interaction of graphene and substrate is strong, has significantly softened phonon dispersion (98). 


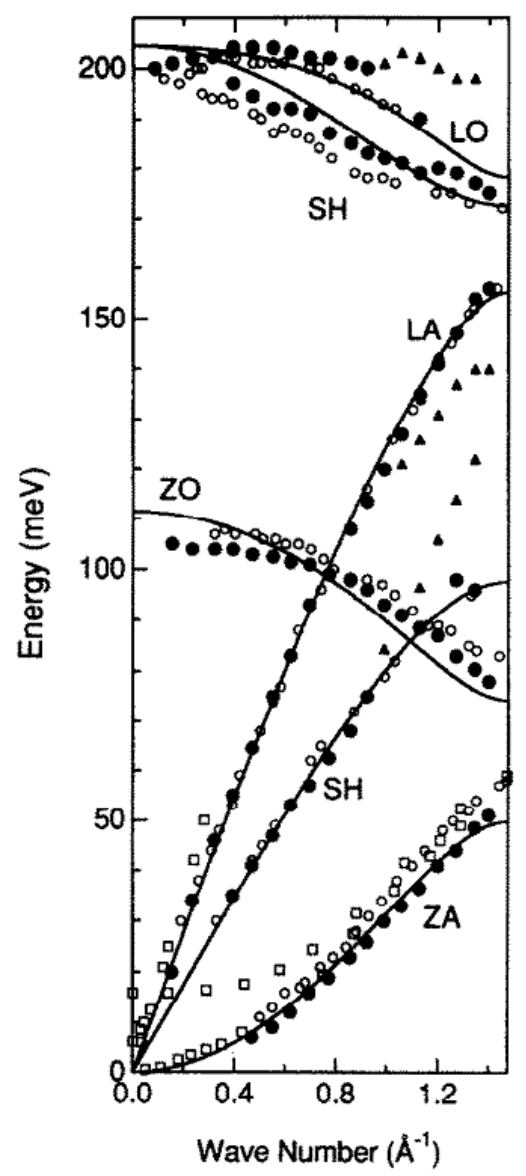

Figure 5.9: Phonon dispersion along the $\Gamma$ to $M$ direction of graphene on Pt(111) compared with that of graphite. The solid circles represent the phonon dispersion of graphene on Pt(111) measured by EELS. The open circles represent phonon dispersion of graphite measured by EELS. The open squares represent the phonon dispersion of graphite measured by neutron scattering. The data points in triangles are from directions other than $\Gamma$ to $M$. The black curves are the calculated dispersion based on the phonon dispersion model described in chapter 5.1. Reprinted from Ref. (90). Copyright (1992) with permission from Elsevier.

Graphene electronic structure: The LEED spectrum and the phonon dispersion indicate a weak interaction of epitaxial graphene and $\mathrm{Pt}(111)$ substrate. Near edge $\mathrm{X}$-ray absorption fine structure (NEXAFS) measurement of epitaxial graphene on several metal substrates also demonstrates that the interaction of graphene and $\operatorname{Pt}(111)$ is one of the weakest $(95,96)$. The direct indication of the interaction strength is to measure the electronic band structure with ARPES. 

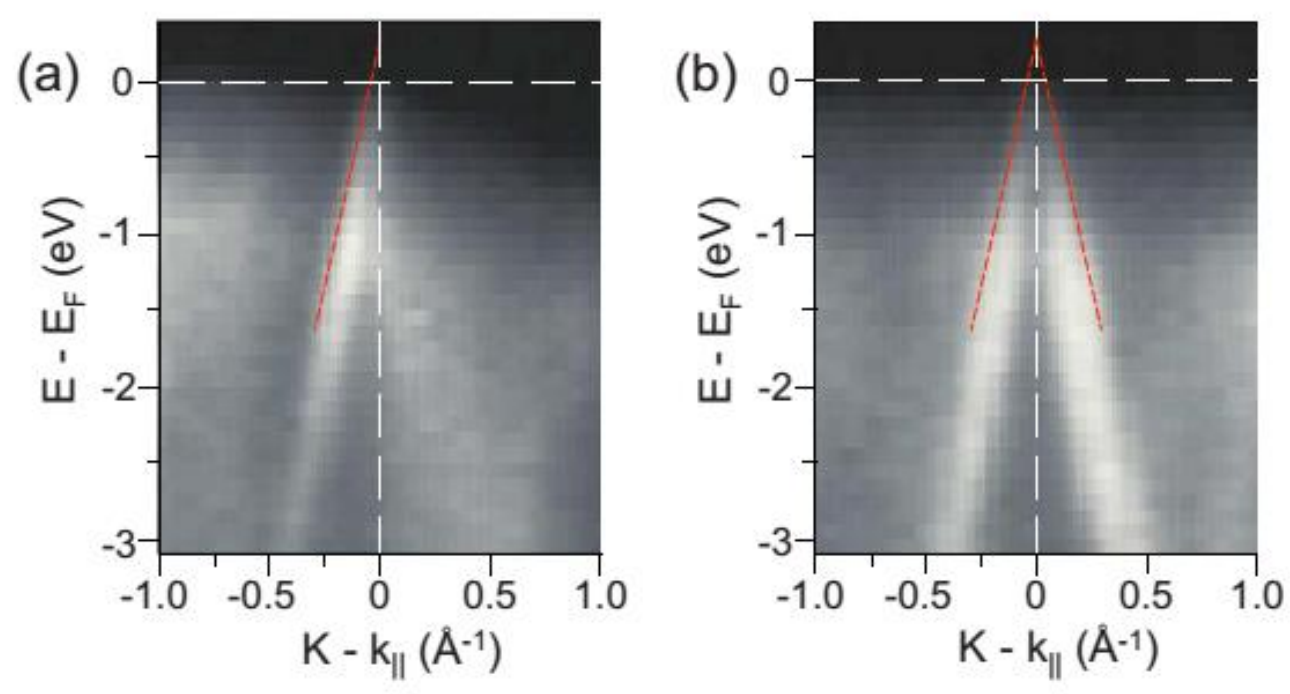

Figure 5.10: Micro-ARPES measurement near the K point of epitaxial graphene on Pt(111). (a). Spectrum along direction $\Gamma$ to $K$. (b). Spectrum along direction perpendicular to $\Gamma$ to $K$. The red lines are guides to the eye, with the slope corresponding to the Fermi velocity of pristine graphene. Reprinted figure with permission from Ref. (89). Copyright (2009) by the American Physical Society

The micro-ARPES measurement of epitaxial graphene on $\operatorname{Pt}(111)$ is shown in Figure 5.10. Panel (a) is measured along $\Gamma$ to $\mathrm{K}$ and (b) is along direction perpendicular to $\Gamma$ to $\mathrm{K}$. The measurement shows linear $\pi$ band dispersion with a Fermi velocity close to $10^{6} \mathrm{~m} / \mathrm{s}$, as expected for pristine graphene. However, the Dirac point is above the Fermi level, implying that the graphene layer is hole doped. Extrapolation of the linear dispersion gives an estimate band crossing at $0.30 \pm 0.15$ eV above Fermi level. Measurements on different graphene domains having different Moiré structures show no difference in band structure and Dirac point energy. Furthermore, the difference between the Pt bands with and without graphene is negligible. These factors confirm the absence of any significant hybridization of graphene $\pi$ band with Pt $d$ band. The weak interaction and shifted Fermi level are also confirmed by several theoretical studies $(93,94)$. 


\subsection{Graphene interaction with hydrogen atoms: Previous theories and experiments}

Hydrogen atoms interaction with graphene has been studied for a long time. In 1970s, D. Hollenbach et al. proposed that $\mathrm{H}$ atoms adsorption on graphitic systems should play an important role in $\mathrm{H}_{2}$ formation in the interstellar space, inspiring a substantial amount of research in the astrophysical community $(64,99,100)$. Hydrogen adsorption on graphene was studied as a possible system for hydrogen storage (9). Recently, it was found that patterned adsorption of hydrogen atoms on graphene can induce a tunable band gap and control the magnetism of the sample, triggering a new series of studies $(68,69,101-103)$. In this subsection, I will briefly introduce existing studies on hydrogen atoms interaction with graphene.

\subsubsection{Energetic and structural studies of $H / D$ atoms adsorption on graphene}

It had been generally accepted that hydrogen atom does not chemisorb on the graphene terrace, but rather at edges and defects, until Sidis et al. for the first time used DFT to treat this problem (104). DFT calculations predict that hydrogen atoms can chemisorb to a pristine graphene plane with an adsorption barrier around $0.2 \mathrm{eV}$ and a binding energy around $0.7 \mathrm{eV}$. Figure 5.11 shows a simple scenario of how hydrogen atoms interact with clean graphene surface based on DFT calculation $(105,106)$. The PW91 functional and a $3 \times 3$ graphene slab were used in this calculation. The calculation gives commonly accepted features of $\mathrm{H}$ atom adsorption on pristine graphene. Figure 5.11 is a 2D PES of an $\mathrm{H}$ atom collinearly colliding with a graphene $\mathrm{C}$ atom at normal incidence. $z_{H}$ is the distance of the $H$ atom from the surface. $z_{C}$ is the height of the bonding $\mathrm{C}$ atom above the graphene plane. Other coordinates of the system are relaxed to the minimum energy configuration. Different colors represent potential energies at different configurations. The figure shows that there is a physisorption well and a chemisorption well with an adsorption barrier in between. The blue curve represents the minimum energy path (MEP) connecting the physisorption well (red spot in the figure), the transition state (blue point in the figure) and the chemisorption well. Formation the $\mathrm{C}-\mathrm{H}$ bond causes reconstruction of the graphene surface, because of the change of hybridization of the binding carbon atom from $\mathrm{sp}^{2}$ to $\mathrm{sp}^{3}$. In the calculation shown in Figure 5.11, the $\mathrm{C}$ atom must pucker out about $0.43 \AA$ from the graphene plane to reach the minimum energy configuration, corresponding to a chemisorption well around $0.76 \mathrm{eV}$ deep. 


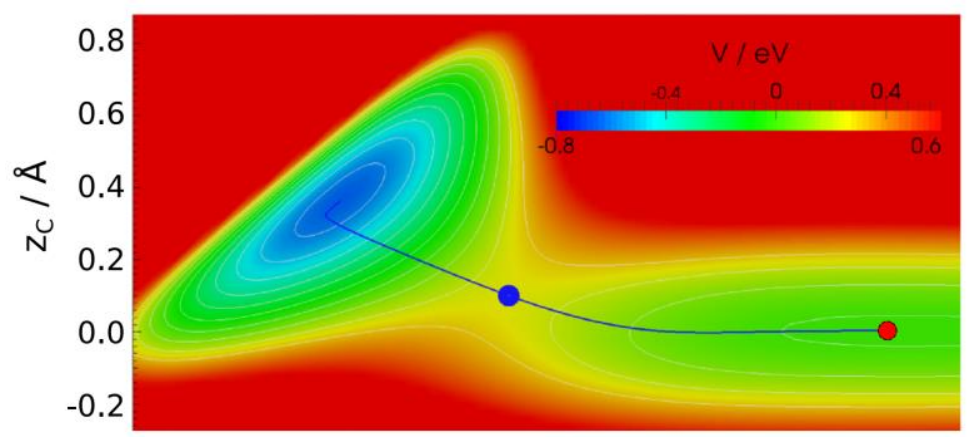

Figure 5.11: PES of single $H$ atom adsorption on graphene. $z_{H}$ and $z_{C}$ are the distances of the $H$ atom and the binding $C$ atom above the surface. The red point indicates the physisorption well. The blue point represents the transition state (TS). The blue curve indicates the minimum energy path (MEP) from the physisorption well to the chemisorption well. Reprinted from Ref. (105), with the permission of AIP Publishing.

There have been large number of calculations on the energetics and minimum energy geometry of a hydrogen atom adsorption on graphene. All calculated PESs look qualitatively similar. For single $\mathrm{H}$ atom adsorption on pristine graphene, GGA level DFT calculations usually predict an adsorption barrier of between 0.2 and $0.3 \mathrm{eV}$, and a chemisorption well about $0.7 \mathrm{eV}$ deep (105119). However, DFT-D calculations, including vdW interactions, give much lower adsorption barriers (120). The wave function method $\operatorname{CCSD}(\mathrm{T})$ predicts an adsorption barrier of $0.4 \mathrm{eV}$ and a chemisorption well of about $0.6 \mathrm{eV}$ for a $\mathrm{H}$ atom adsorption on the coronene molecule (39). Unfortunately there has been no direct experimental measurement of the adsorption barrier height and bond strength for a $\mathrm{H}$ atom adsorption on pristine graphene. T. Zecho et al. experimentally verified that $\mathrm{H}$ atoms can chemisorb on a graphene surface $(121,122)$. Their temperature dependent HREEL spectroscopy measurement clearly showed the formation of the $\mathrm{C}-\mathrm{H}$ bond and the $\mathrm{C}-\mathrm{H}$ bond vibrational frequency at $2650 \mathrm{~cm}^{-1}$ for stretching and $1210 \mathrm{~cm}^{-1}$ for bending. E. Aréou et al. used HREEL spectroscopy and low kinetic energy $\mathrm{H}$ atom beam to confirm the existence of a barrier (123), but did not provide an exact value. In contrast, the physisorption well has been measured to be $40 \mathrm{meV}$ by $\mathrm{H}$ atom diffraction resonance (33), a value that was later confirmed by wave function based calculations $(38,124)$. 

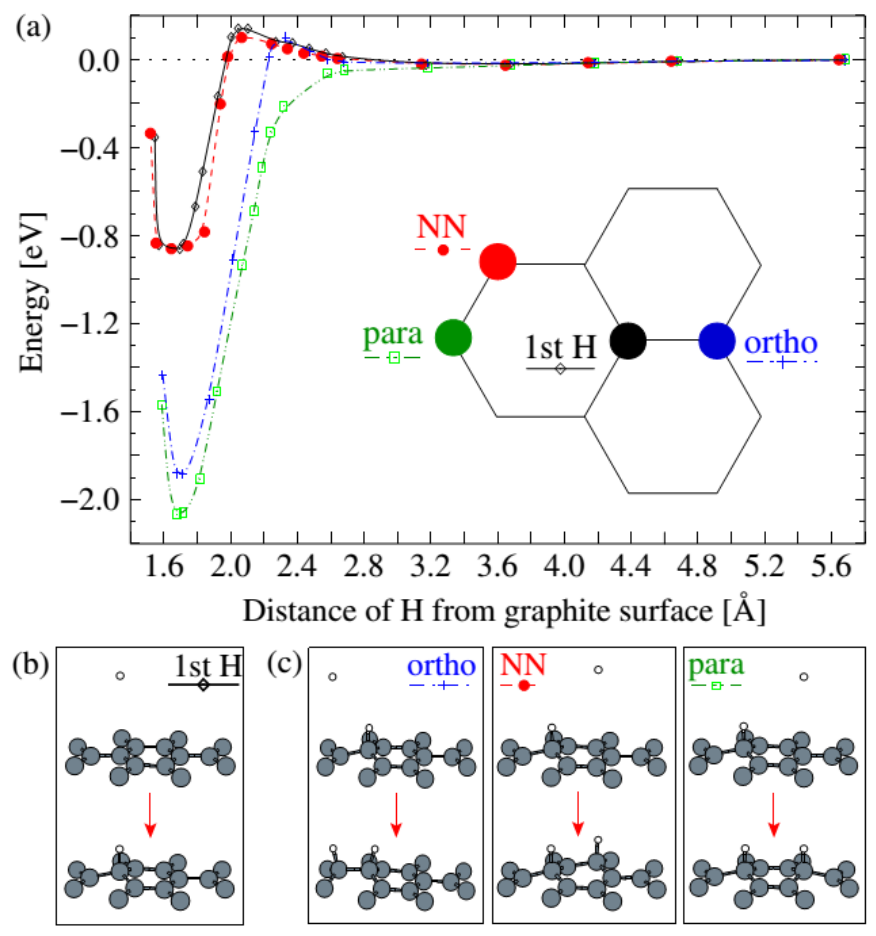

Figure 5.12: Upper panel: Potential energy curves (PECs) obtained from DFT calculations for hydrogen dimer formation on graphene. The black curve is the PEC for the adsorption of a single $H$ atom on clean graphene. The blue, green and red curves are PECs for the formation of different dimer configurations indicated in the figure. Lower panel: structures of the monomer and different dimer configurations. Reprinted figure from Ref. (125). Copyright (2006) by the American Physical Society.

Both theory and experiment have confirmed that adsorption of $\mathrm{H}$ atoms on graphene is a coverage dependent process $(110-112,125,126)$. Figure 5.12 shows the potential energy curves of different dimer formations. Note that the addition of a second $\mathrm{H}$ atom to the $\mathrm{NN}$ site gives rise to a similar potential curve as that for the adsorption of the first $\mathrm{H}$ atom. On the other hand, addition of a second atom to the para site gives rise to a very different potential energy curve that involves no barrier and results in a stronger bond. Adsorption onto the ortho site lies in between the above two cases (125). This preferential sticking at the para and ortho sites can be rationalized by the lattice structure of graphene. Graphene is comprised of two equivalent sublattices. Once the $\mathrm{H}$ atom sticks onto the first sub-lattice, the resulting unpaired electron delocalizes in the second sub-lattice, leading to a reduced barrier height and stronger bond on the 
second sub-lattice (112). Preferential sticking was also observed on epitaxial graphene due to the interaction of graphene and its supporting substrate (96) and has been used to manipulate the adsorption pattern (127-129). Unlike the lack of experimental studies on the first adsorption event, there have been large amounts of experimental work on the patterned adsorption of $\mathrm{H}$ atoms on graphene. It is mainly motived by the formation of $\mathrm{H}_{2}$ on graphitic surfaces and chemical functionalization of graphene $(68,69,96)$. Preferential pair adsorptions of $\mathrm{H}$ atoms on graphene as is shown in Figure 5.12 have been verified by STM studies at coverage even as low as $1 \%(125,126,130,131)$. Controlled adsorption patterns on epitaxial graphene on various substrates have been confirmed by various experimental methods, including X-ray photoelectron spectroscopy, X-ray adsorption spectroscopy, temperature programmed desorption and scanning tunneling microscopy $(96,103,126-129)$. Samples of graphene that have undergone different patterns of Hydrogenation exhibit various interesting electronic, magnetic and thermal properties that are employed in graphene applications.

\subsubsection{Studies of dynamics of hydrogen atoms adsorption on graphene}

Due to experimental difficulties, the studies of dynamics of hydrogen atom adsorption on graphene have so far been primarily theoretical. Many models have been proposed to describe the dynamical features of the adsorption process. In these calculations, the system is usually divided into two interacting sub-systems, which are the $\mathrm{C}-\mathrm{H}$ bonding sub-system and graphene lattice sub-system. Owing to the computational demand explained in chapter 2, the two subsystems cannot be treated simultaneously in full dimension with ab initio accuracy. The smaller C-H sub-system can usually be treated more accurately. The potential energy surface of the $\mathrm{C}-\mathrm{H}$ system is usually generated by fitting the results of relaxed DFT calculations $(105,106$, 108, 109, 111). The C-H motion is treated quantum mechanically or classically. For quantum mechanical descriptions of the $\mathrm{C}-\mathrm{H}$ bond, calculations are usually carried out in reduced dimensionality such as $1 \mathrm{D}$ (only $\mathrm{H}$ movement parallel to the surface normal) or $2 \mathrm{D}(\mathrm{H}$ and $\mathrm{C}$ movements parallel to the surface normal) $(115,116,132)$. For classical description, more degrees of freedom can be included $(109,111)$. The graphene lattice is usually described by empirical potentials such as the model shown in chapter 5.1. Pure classical descriptions or quantum mechanical oscillator models have been adapted to treat the graphene lattice motion. The interaction between the $\mathrm{C}-\mathrm{H}$ bond sub-system and the graphene lattice is usually described 
by the coupling of the $\mathrm{C}$ atom in the $\mathrm{C}-\mathrm{H}$ bond to the normal modes of the graphene lattice (105, 106, 133, 134). Models using different approximations of the system usually provide different results. Using a perturbative coupling model, Sakong et al. calculated the vibrational lifetime of C-H and C-D stretching on graphene to be 5.2 ps and 46.8 ps, respectively. The lifetime difference in $\mathrm{H}$ and $\mathrm{D}$ is rationalized by the coupling order and the graphene phonon density of states at the frequencies resonant with the stretching mode $(133,134)$. Sha et al. treated the C-H system with a 4D potential energy surface and a reduced graphene phonon bath mode. Both their quantum and classical calculations give a low trapping probability of 5\%-10\% for a normal incidence thermal $\mathrm{H}$ atom beam $(2000 \mathrm{~K})(108,109$, 111). AIMD calculations performed by Stephan et al. also predicted a low sticking probability (127). Morisset et al. developed a simplified 1D quantum description of the $\mathrm{C}-\mathrm{H}$ sub-system, but used a more realistic description of the phonon bath. Large trapping probabilities of several tens of percent were obtained (115, 116). Recently, more sophisticated models describing the $\mathrm{C}-\mathrm{H}$ system with a high-dimensional potential and including a large quantum phonon bath are being developed.

In conclusion, substantial amount of theoretical progresses have been achieved on the $\mathrm{H}$ atom adsorption on graphene, yet not much of experimental work has been done. Based on the facts discussed in Section 5.3.1, accurate experimentally measured adsorption barrier height, especially the zero coverage value, can be taken as a benchmark to validate different theoretical approaches. Experimentally determined energy transfer, again especially at the zero coverage limit, can greatly improve the theoretical modeling. In the next chapter, I will present the most recent experimental studies of the adsorption barrier height and dynamics of hydrogen atoms on epitaxial graphene on $\operatorname{Pt}(111)$. 


\section{Hydrogen Atoms scattering from Epitaxial Graphene on Pt(111): Adsorption Threshold and Energy Transfer}

As shown in Chapter 5, adsorption of hydrogen atoms on graphene is a chemically activated process. Formation of the $\mathrm{C}-\mathrm{H}$ bond causes reconstruction of the graphene surface, because of the change of hybridization of the binding carbon atom from $\mathrm{sp}^{2}$ to $\mathrm{sp}^{3}$. The change in the electronic structure and geometry induced by the adsorption leads to a barrier. Significant progress has been made in understanding adsorption of $\mathrm{H}$ atoms on graphene. Yet, this problem is far from being fully understood. First, accurate experimentally obtained adsorption threshold (minimum normal incidence energy required for the hydrogen atoms to cross the adsorption barrier) does not exist. Different theoretical methods predict significantly different barrier heights $(39,106,112,120)$. Accurately measured experimental values could provide a benchmark to validate theoretical models. Second, there has been no experimental investigation on the dynamics of adsorption of $\mathrm{H}$ atoms on graphene. Hydrogen atoms may experience different dynamics before and after they cross the adsorption barrier. The choice of applied energy dissipation description gives rise to significant differences in the calculated adsorption probabilities $(108,109,111,115,116)$. Third, zero surface coverage experimental measurements for studying the initial hydrogen atom sticking are still missing. Hydrogen dimers and clusters could form even at coverage as low as $1 \%(126,131,135)$, and their adsorption energetics and dynamics are significantly different from the initial sticking $(110,112,127)$.

Previous experimental studies of $\mathrm{H}$ atom adsorption on graphene have primarily involved thermal $\mathrm{H}$ atom beams of about $2000 \mathrm{~K}-3000 \mathrm{~K}$ and high surface coverage $(121,125,127,128$, 131). Due to the uncertainties in the translational energy and surface coverage, these experimental results do not provide conclusive information about the dynamics and energetic landscape of the zero-coverage adsorption event. In this chapter, I will present the experimental results of monoenergetic $\mathrm{H}$ atom beam scattering from epitaxial graphene on $\mathrm{Pt}(111)$ at zero surface coverage. The method used to generate monoenergetic $\mathrm{H}$ atom beams has been described in chapter 3. Low beam fluxes $\left(10^{8} \sim 10^{9}\right.$ atoms $\left./ \mathrm{cm}^{2} \cdot \mathrm{s}\right)$ ensure zero surface coverage during the measurements. Narrow energy spread makes it possible to predict zero-coverage H/D adsorption thresholds with small errors. The energy dissipation of H/D atoms before and after the C-H/D bond formation is studied via high-resolution Rydberg atom tagging time-of-flight measurements. 
As an example of $\mathrm{H}$ atom scattering on semiconductor surfaces, the translational energy loss and scattering angle distributions are compared to those on metals.

This chapter is organized as follows. In Section 6.1, the comparison between $\mathrm{H}$ atoms scattering from pristine $\mathrm{Pt}(111)$ and from epitaxial graphene on $\mathrm{Pt}(111)$ is presented. Section 6.2 includes detailed experimental results of H/D atoms scattering from epitaxial graphene under various incidence conditions. Translational energy and scattering angle distributions for the scattered atoms are measured as a function of total incidence energy, incidence angle and surface temperature, respectively. In Section 6.3, the adsorption thresholds for $\mathrm{H}$ and $\mathrm{D}$ atoms on epitaxial graphene are determined (minimum normal incidence energy required for the hydrogen atoms to cross the adsorption barrier). In Section 6.4, I discuss the dynamics of H and D atoms scattering on epitaxial graphene with and without crossing the adsorption barrier. 


\subsection{Comparison between hydrogen atoms scattering from epitaxial graphene on $\operatorname{Pt}(111)$ and pristine $\operatorname{Pt}(111)$}

In this section, translational energy loss and scattering angle distributions for $\mathrm{H}$ atoms scattering from epitaxial graphene on $\mathrm{Pt}(111)$ and pristine $\mathrm{Pt}(111)$ are compared. Tunable monoenergetic $\mathrm{H}$ atom beams generated by laser photolysis of hydrogen halides are scattered from well-defined epitaxial graphene and $\operatorname{Pt}(111)$ surfaces. Incidence angle of the atom beams is adjusted by titling the surface sample holder. Scattering angle resolved time-of-flight distributions of the scattered atoms are measured by Rydberg atom tagging scheme. The experimental setup and methods are the same as those discussed in Chapter 3 and 4. The experimental geometry is shown in Figure 3.15 and 4.1. The graphene sheet was grown on a clean Pt(111) substrate in our UHV chamber. The synthesis and characterization procedures are described in Section 5.2.
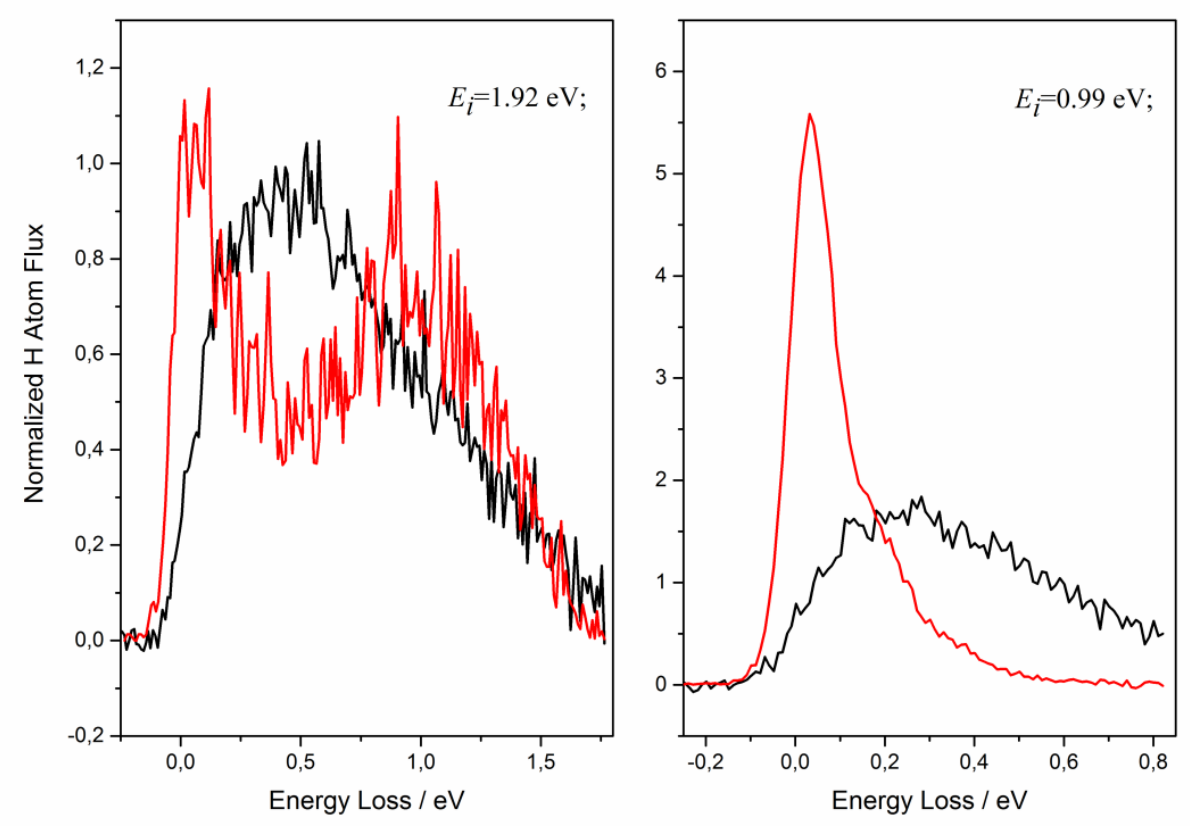

Figure 6.1: Translational energy loss distribution of $H$ atoms scattering from epitaxial graphene and clean Pt(111) surface. The incidence angle and scattering angle are both $45^{\circ}$. The incidence energy is $1.92 \mathrm{eV}$ for the left panel and $0.99 \mathrm{eV}$ for the right one. Black curves represent scattering from clean Pt(111) surface. Red curves represent scattering from epitaxial graphene.

Figure 6.1 shows the translational energy loss distributions of $\mathrm{H}$ atoms scattering from epitaxial graphene and clean $\operatorname{Pt}(111)$ surface. Black curves represent scattering from clean $\operatorname{Pt}(111)$ at 
incidence energies of $1.92 \mathrm{eV}$ and $0.99 \mathrm{eV}$. The translational energy loss distributions of $\mathrm{H}$ atoms scattering from $\mathrm{Pt}(111)$ share the same features as scattering from $\mathrm{Au}(111)$ shown in chapter $4 . \mathrm{H}$ atoms translational energy loss is large and shows a broad distribution, due to the dominant role of non-adiabatic electronic excitations. The average energy loss, normalized to the incidence energy for scattering from $\mathrm{Pt}(111)$, is about 0.33, which is the same as that for $\mathrm{H}$ atoms scatterings from $\mathrm{Au}(111)$. The energy loss distributions for $\mathrm{H}$ atoms scattering from epitaxial graphene on $\operatorname{Pt}(111)$ are significantly different from the distributions obtained from scattering on clean $\operatorname{Pt}(111)$. The energy loss distribution at $0.99 \mathrm{eV}$ incidence energy is narrow and peaks at a small value $(0.03 \mathrm{eV})$. In contrast, the energy loss distribution at $1.92 \mathrm{eV}$ incidence energy exhibits a bimodal feature.

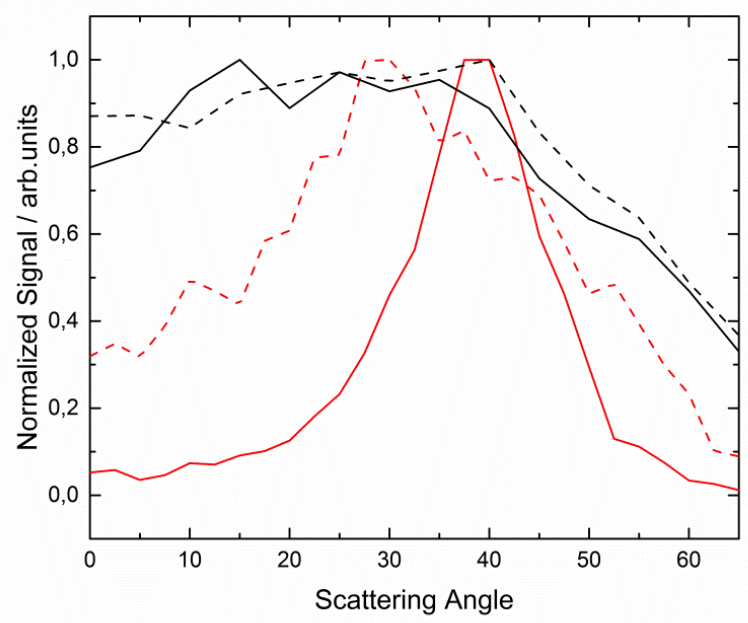

Figure 6.2: Scattering angle distribution of $H$ atoms scattering from epitaxial graphene on Pt(111) and clean Pt(111). Incidence angles are all $45^{\circ}$. Dashed curves and solid curves represent results at incidence energy of $1.92 \mathrm{eV}$ and $0.99 \mathrm{eV}$, respectively. Black and red curves represent results from scattering from clean Pt(111) and epitaxial graphene, respectively.

Figure 6.2 shows angular distributions of $\mathrm{H}$ atoms scattering from epitaxial graphene on $\operatorname{Pt}(111)$ and clean $\operatorname{Pt}(111)$. Black and red curves represent scattering from clean $\operatorname{Pt}(111)$ and epitaxial graphene, respectively. Dashed and solid curves represent scattering at incidence energies of $1.92 \mathrm{eV}$ and $0.99 \mathrm{eV}$, respectively. The scattering angle distributions for $\mathrm{Pt}(111)$ are again the same as those for $\mathrm{Au}(111)$. Scattering angles for both incidence energies have a broad 
distribution, which is due to the fact that there is a distribution in the number of bounces (typically 1-3 times) that the $\mathrm{H}$ atom makes. $\mathrm{H}$ atoms scattering on graphene at $0.99 \mathrm{eV}$ incidence energy exhibit a narrow angular distribution (FWHM $15^{\circ}$ ) and peak at $40^{\circ}$. Scattering of $1.92 \mathrm{eV} \mathrm{H}$ results in a broader angular distribution (FWHM $30^{\circ}$ ) but still has a preferential direction (peaks at $30^{\circ}$ ).

The fact that the energy loss and scattering angle distributions obtained from the $\mathrm{H}$ on graphene and the $\mathrm{H}$ on $\operatorname{Pt}(111)$ systems are qualitatively different indicates that there are qualitative differences in the dynamics that occur in these two systems. The clear differences also prove that the epitaxial graphene layer completely covers the $\operatorname{Pt}(111)$ substrate, since no features of scattering from $\mathrm{Pt}(111)$ is seen for the scattering measurements from graphene.

Figure 6.1 and 6.2 also show that $\mathrm{H}$ atoms scattering from epitaxial graphene strongly depend on the incidence energies. Increasing the total incidence energy from $0.99 \mathrm{eV}$ to $1.92 \mathrm{eV}$ drastically changes the scattering angle and energy loss distributions. This change induced by increasing incidence energy may involve different dynamics of $\mathrm{H}$ atoms scattering from graphene with and without crossing the adsorption barrier.

\subsection{H/D atoms scattering from epitaxial graphene on $\mathrm{Pt}(111)$ under various incidence conditions}

As shown in Section 6.1, $\mathrm{H}$ atoms scattering from epitaxial graphene has strong dependence on total incidence energy, which may involve different dynamics of $\mathrm{H}$ atoms scattering from graphene with and without crossing the adsorption barrier. In this section, I present studies of $\mathrm{H}$ atoms scattering from epitaxial graphene as a function of total incidence energy, incidence angle (corresponds to normal incidence energy) and surface temperature, respectively. Isotope effects (changing $\mathrm{H}$ to $\mathrm{D}$ ) of the scattering are also studied. In Section 6.2.1, the dependences of $\mathrm{H}$ atoms scattering from graphene on the total incidence energy are presented. Section 6.2.2 includes results of $\mathrm{H}$ atoms scattering from graphene as a function of incidence angle. The reason for this set of measurement is that normal incidence energy, not total incidence energy, is directly related to barrier crossing. In Section 6.2.3, the dependence of $\mathrm{H}$ atoms scattering from epitaxial graphene on surface temperature is presented. Isotope effects of the scattering are shown in Section 6.2.4. 
For every incidence conditions (including incidence energy $\left(E_{\mathrm{i}}\right)$, incidence angle $\left(\theta_{\mathrm{i}}\right)$, surface temperature and atom species), time-of-flight distributions are measured at scattering angles $\left(\theta_{\mathrm{s}}\right)$ between $0^{\circ}$ to $65^{\circ}$ in $2.5^{\circ}$ per step. The angular acceptance of our measurement is $3^{\circ}$. The measurement limitation of $0^{\circ}-65^{\circ}$ arises from the constraints in the experimental setup. Only inplane data are recorded in the experiments. Figure 6.3 shows an example of the two dimensional scattering angle and translational energy distribution of $\mathrm{H}$ atoms scattered from graphene. The radial axis represents the translational energy of scattered $\mathrm{H}$ atoms $\left(E_{\mathrm{s}}\right)$ normalized to the incidence energy $\left(E_{\mathrm{i}}\right)$. The polar axis represents the scattering angle (with data) and incidence angle (blank). The gray bar indicates the epitaxial graphene on $\operatorname{Pt}(111)$. The long dashed red arrow in the figure indicates the incidence angle $\left(\theta_{\mathrm{i}}\right)$. The short red arrow indicates the specular angle $\left(\theta_{\text {is }}\right)$ and zero degree corresponds to the surface normal. The scattering probability density $\left(\mathrm{P}\left(\mathrm{E}_{\mathrm{s}} ; \theta_{\mathrm{s}}\right)\right)$ is displayed via the color coding. The two-dimensional distribution is normalized to the integrated area.

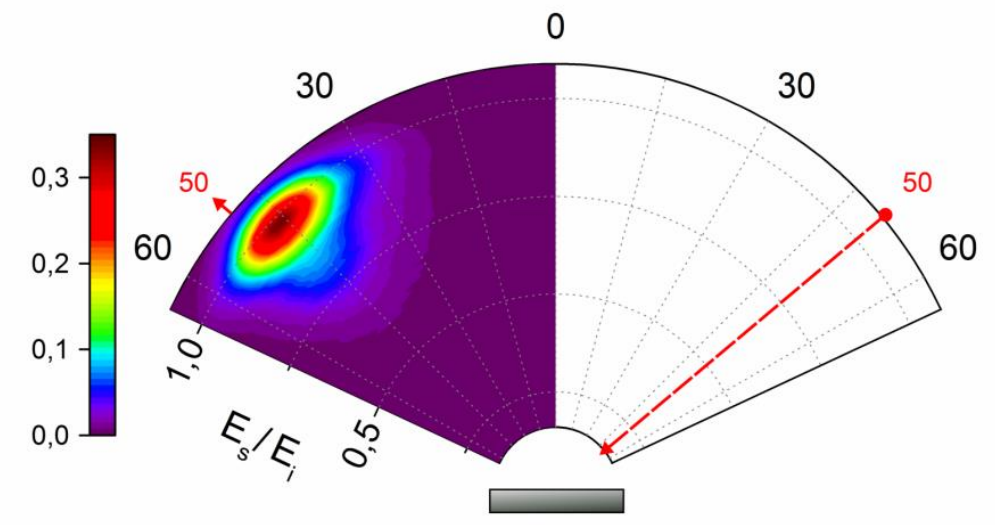

Figure 6.3: Two-dimensional scattering angle and translational energy distribution of $H$ atoms scattered from graphene. The long red dashed arrow indicates the incidence angle. The short red solid arrow indicates the specular angle. The gray bar indicates the epitaxial graphene on Pt(111). Zero degree corresponds to the surface normal. The radial axis represents the translational energy of scattered $H$ atoms $\left(E_{s}\right)$ normalized to the incidence energy $\left(E_{i}\right)$. The polar axis represents the scattering angle (with data) and incidence angle (blank). The scattering probability density $\left(P\left(E_{s} ; \theta_{s}\right)\right)$ is displayed via the color coding. The two-dimensional distribution is normalized to the integrated area. 


\subsubsection{H atoms scattering from epitaxial graphene as a function of incidence energy}

Figure 6.4 shows the $2 \mathrm{D}$ translational energy and scattering angle distribution $\left(P\left(E_{\mathrm{s}} ; \theta_{\mathrm{s}}\right)\right)$ of $\mathrm{H}$ atoms scattered from epitaxial graphene at different incidence energies $\left(E_{\mathrm{i}}\right)$ but constant incidence angle $\left(\theta_{\mathrm{i}}\right)$ of $50^{\circ}$. The incidence energies are $0.99 \mathrm{eV}, 1.92 \mathrm{eV}, 2.18 \mathrm{eV}, 2.62 \mathrm{eV}$ and $3.31 \mathrm{eV}$ for Figure 6.4 (a)-(e) respectively. The radial axis represents the translational energy of scattered atoms $\left(E_{\mathrm{s}}\right)$ normalized to the incidence energy $\left(E_{\mathrm{i}}\right)$. The polar axis represents the scattering angles $\left(\theta_{\mathrm{s}}\right)$, with a red bar indicating the specular angle $\left(\theta_{\mathrm{is}}\right)$. The $P\left(E_{\mathrm{s}} ; \theta_{\mathrm{s}}\right)$ are normalized to the integrated area and shown via color coding. Clear differences in the $P\left(E_{\mathrm{s}} ; \theta_{\mathrm{s}}\right)$ can be seen for different incidence energies. At low $E_{\mathrm{i}}$ of $0.99 \mathrm{eV}$, the scattering signal is nearelastic and near-specular. The scattering signal peaks at an energy about $30 \mathrm{meV}$ less than $E_{\mathrm{i}}$, and at an angle $5^{\circ}$ less than the specular angle. Both the energy and angular distributions are narrow, indicating the event is like direct scattering on a hard wall. As the incidence energy is increased to $1.92 \mathrm{eV}$, the near-elastic and near-specular fast component decreases and a slow component with broader energy and angular distribution emerges. In this thesis we will call the near-elastic and near-specular component as "fast component" and the other component as "slow component". For $1.92 \mathrm{eV}$ incidence energy, the slow component peaks at about half of the incidence energy $(1 \mathrm{eV})$, and about $30^{\circ}$ away from the surface normal. The FWHM for the energy and angular distributions of the slow component are about $1 \mathrm{eV}$ and $30^{\circ}$, respectively. As the incidence energy is further increased, the fast component decreases, and the slow component increases. When the incidence energy is above $2.62 \mathrm{eV}$, the fast component vanishes. The fast component always peaks near the specular angle and with an energy close to the incidence energy, while the slow component always peaks at an angle between $30^{\circ}-35^{\circ}$ off the surface normal and an energy between $40 \% \sim 50 \%$ of the incidence energy. The change in the distribution is caused by increasing the incidence energy, which is a strong indication for that the dynamics involve a barrier crossing. 

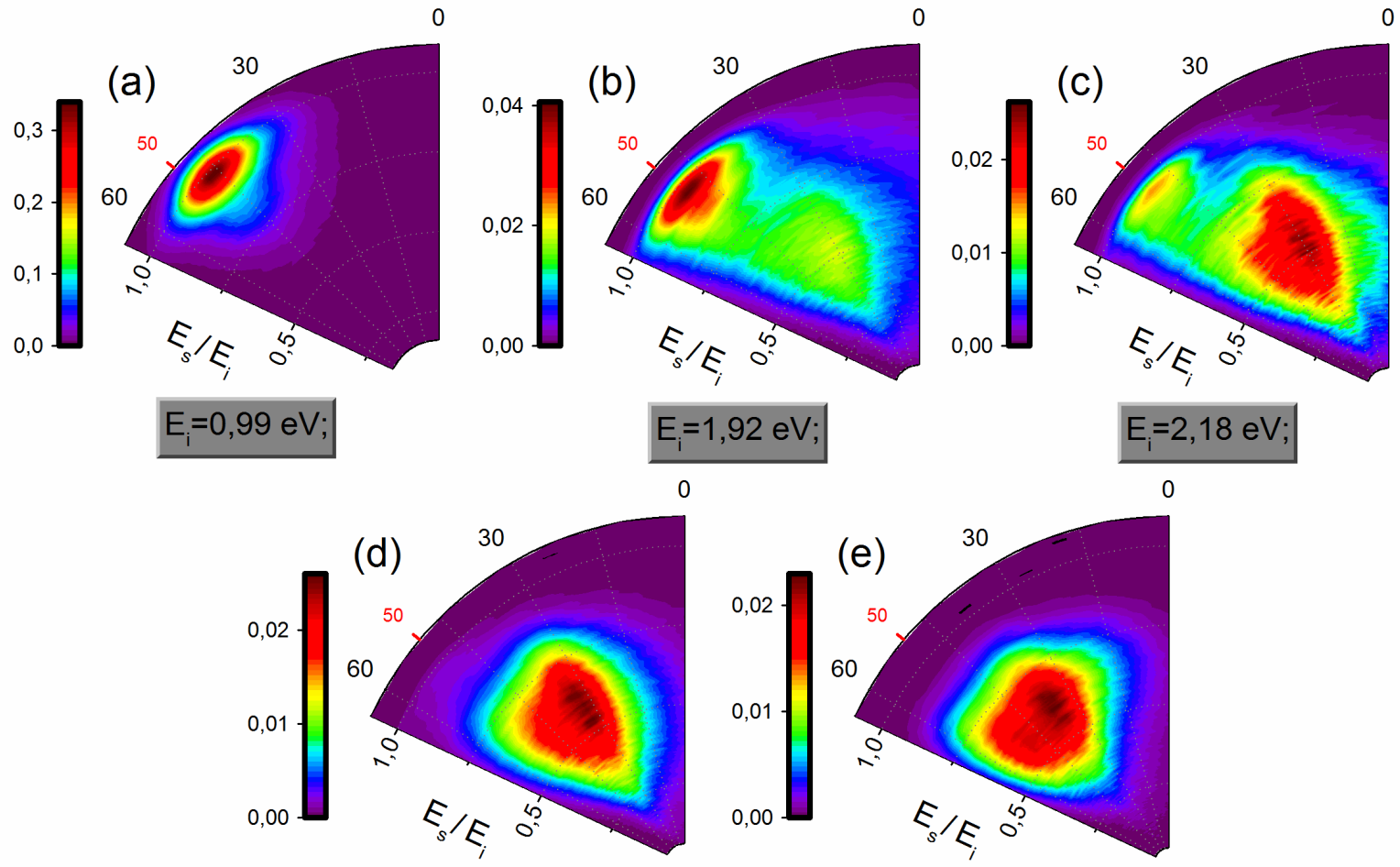

0 $E_{i}=2,18 \mathrm{eV}$; $E_{i}=2,62 \mathrm{eV}$;

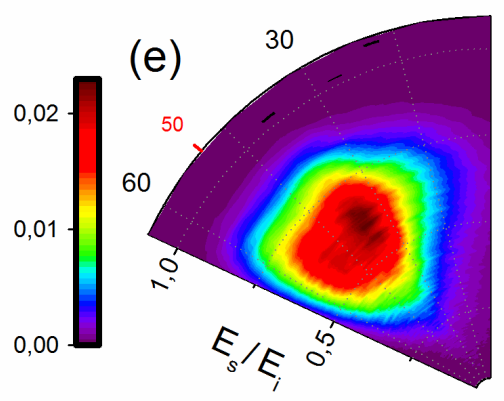

$E_{i}=3,31 \mathrm{eV}$;

Figure 6.4: 2D translational energy and scattering angle distribution of $H$ atoms scattered from epitaxial graphene at different incidence energies but constant incidence angle $\left(50^{\circ}\right)$. The incidence energies are shown in gray bars in the figure.

\subsubsection{H atoms scattering from epitaxial graphene as a function of incidence angle}

In the previous section, it was shown that the 2D translational energy and scattering angle distributions change with incidence energies. A possible reason for this change is that the observed dynamics involve the crossing of an adsorption barrier. A set of scattering experiments at different incidence angles but constant total incidence energy may provide additional support for this assumption, since normal incidence energy, not total incidence energy, is directly related to crossing of an adsorption barrier.

Figure 6.5 shows the results of $\mathrm{H}$ atom scattering from epitaxial graphene at different incidence angles but same incidence energy (equivalent of different normal incidence energy but constant total incidence energy). The incidence energy is $1.92 \mathrm{eV}$. The incidence angle is varied between $0^{\circ}-60^{\circ}$ in $2^{\circ}$ steps as shown by Figure 6.5 (a) - (p). The specular scattering angles are highlighted 
with red ticks. The data were typically recorded at scattering angles between $0^{\circ}$ and $65^{\circ}$. However, it is impossible to detect the full $0^{\circ}-65^{\circ}$ degree scattering range for incidence angles smaller than $40^{\circ}$, due to constraints from the experimental setup. As shown in the figure, our scattering signal is concentrated in scattering angles between $0^{\circ}-65^{\circ}$. Figure 6.5 (a) shows the results at the largest incidence angle of $60^{\circ}$, corresponding to normal incidence energy of 0.48 $\mathrm{eV}$. It vaguely shows a bimodal feature consisting of an intense fast component and a weak slow component. The fast and slow components exhibit translational energy and angular distributions similar to those of the two components described in the earlier Section 6.2.1. As the incidence angle is decreased, the ratio of the slow component to the fast component increases. For incidence angles below $36^{\circ}$, the fast component is not detected. This set of measurements provides additional support for the assumption proposed at the end of earlier Section 6.2.1, that the changing in the 2D distribution of scattered atoms arises from adsorption barrier crossing. As the incidence angle is decreased, more $\mathrm{H}$ atoms have enough normal incidence energy to cross the barrier. When the normal incidence energy is above a certain value, all the $\mathrm{H}$ atoms can overcome the barrier, and the fast component vanishes.

Here, one should note that the term "barrier height" in literature usually denotes the energy of the transition state at the adsorption barrier. It is the saddle point on the multi-dimensional PES, and is the minimum energy required to overcome the barrier as shown in Figure 5.11. The saddle point corresponds to a specific atomic configuration. There exist trajectories that do not cross the saddle point, and these trajectories will experience a higher potential energy. This gives rise of the simultaneous existence of the fast and the slow components for some incidence conditions. 

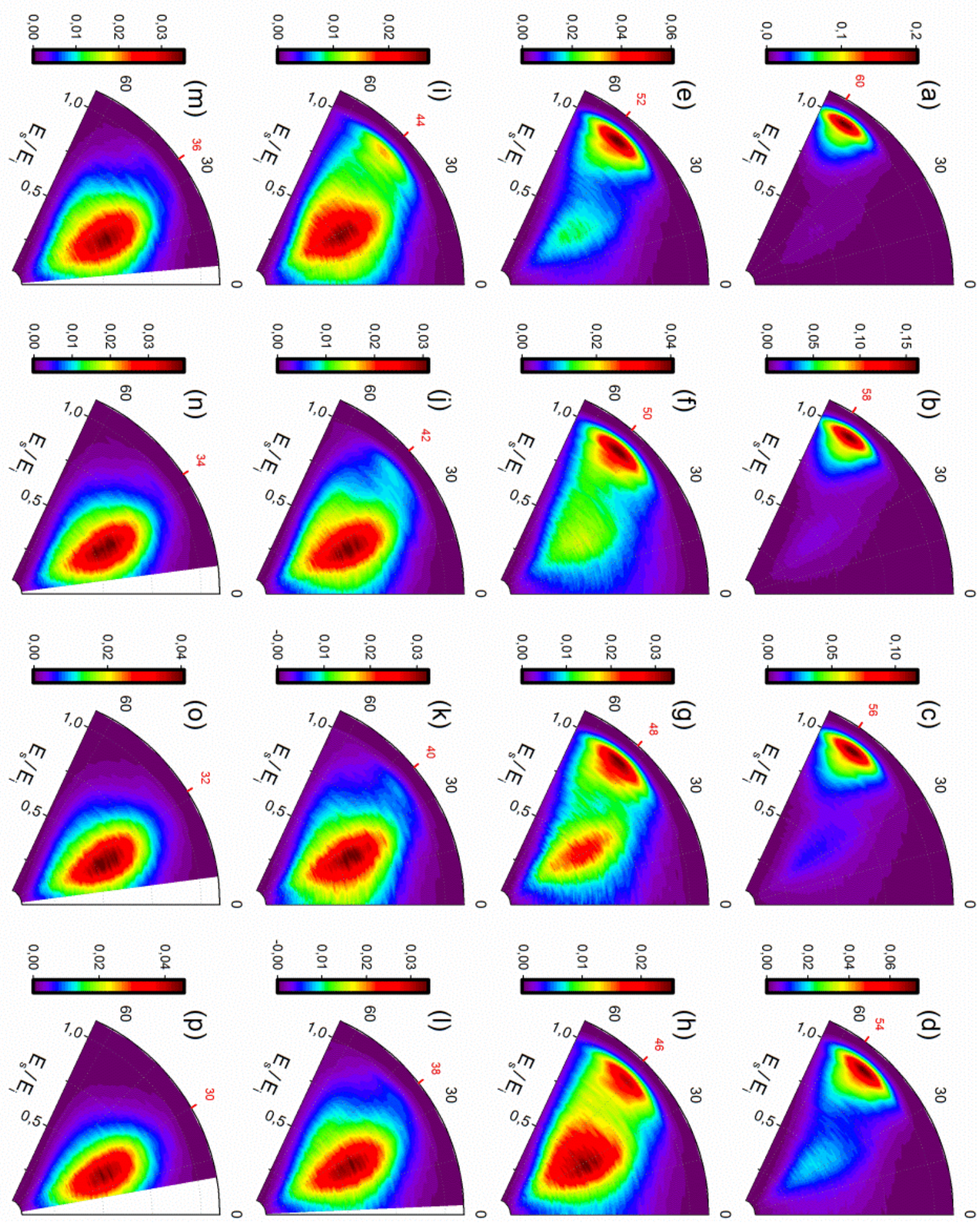

Figure 6.5: $2 D$ translational energy and scattering angle distribution of $H$ atom scattered on epitaxial graphene at different incidence angles but the same incidence energy $(1.92 \mathrm{eV})$. The red ticks represent the specular scattering angle. 


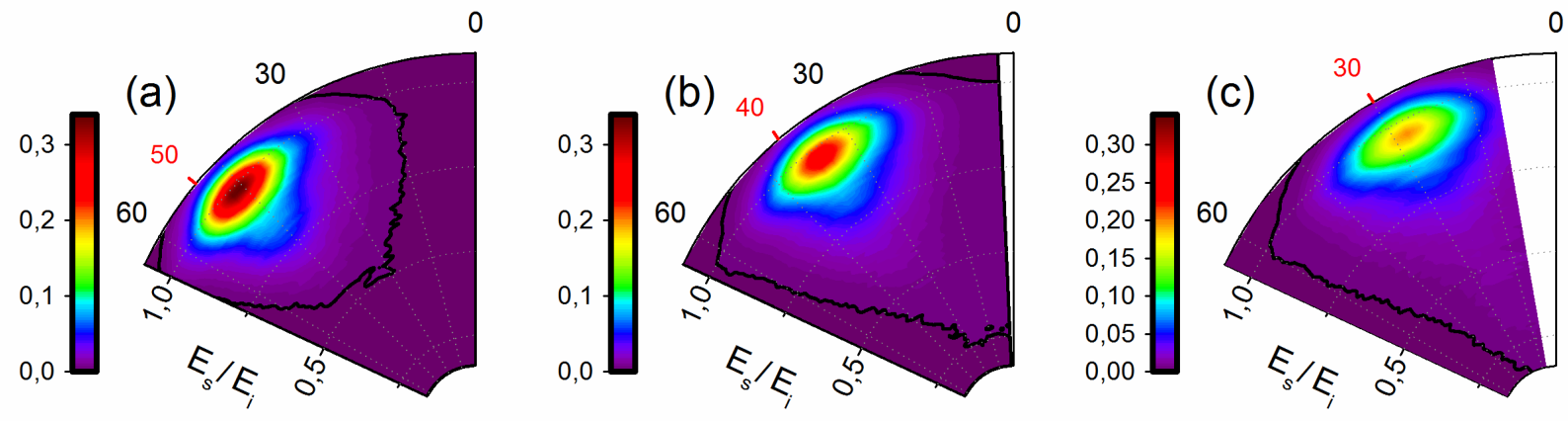

Figure 6.6: 2D translational energy and scattering angle distribution of $H$ atoms scattered from epitaxial graphene at three different incidence angles $\left(50^{\circ}, 40^{\circ}\right.$ and $\left.30^{\circ}\right)$ but same incidence energy $(0.99 \mathrm{eV})$. The red ticks represent the specular scattering angle.

Figure 6.6 shows the results of $\mathrm{H}$ atom scattering on epitaxial graphene at three different incidence angles but the same incidence energy of $0.99 \mathrm{eV}$. The incidence angles are $50^{\circ}, 40^{\circ}$ and $30^{\circ}$, corresponding to normal incidence energies of $0.41 \mathrm{eV}, 0.58 \mathrm{eV}$ and $0.74 \mathrm{eV}$. The black curves in the figures are contour curves, corresponding to $1 \%$ of the peak values. Here, one should note that the color bars have the same scale for all the three panels, which is different from those shown in Figure 6.4 and 6.5 (color bars have different scales with each other).

For scattering at incidence angle of $50^{\circ}(0.41 \mathrm{eV}$ normal incidence energy), there is only a fast peak and no visible scattering signal at energies smaller than half of the incidence energy, indicating that no $\mathrm{H}$ atom crosses the adsorption barrier. For incidence angle at $40^{\circ}(0.58 \mathrm{eV}$ normal incidence energy), the peak value drops $1 / 3$ relative to the scattering at $50^{\circ}$ incidence angle, and scattered atoms appear with energies less than $0.5 \mathrm{eV}$, especially at scattering angles close to the surface normal as illustrated by the black contour line. This indicates that the $\mathrm{H}$ atoms scattered back with low energies are from the barrier crossed scattering. As the incidence angle is increased to $30^{\circ}(0.71 \mathrm{eV}$ normal incidence energy), the peak intensity continues to drop, and the intensity at small energies rises, indicating more $\mathrm{H}$ atoms have enough normal incidence energy to cross the barrier.

The reason for no clear bimodal feature in the distribution is as follows. From figure $6.4-6.6$, it can be clearly seen that the scattered $\mathrm{H}$ atoms in the slow component suffer a large amount of energy loss. For incidence energy of $1.92 \mathrm{eV}$, the scattered $\mathrm{H}$ atoms in the slow component on average lose half of their initial energy. For incidence energy of $0.99 \mathrm{eV}$, the scattered $\mathrm{H}$ atoms 
in the slow component lose $50 \%$ to $80 \%$ of their incidence energy, as indicated by Figure 6.6. It is possible that a portion of the $\mathrm{H}$ atoms that cross the barrier become adsorbed and are not scattered back into the gas phase. The adsorption probability of $\mathrm{H}$ atoms crossing the barrier should be higher at incidence energy of $0.99 \mathrm{eV}$ than that at incidence energy of $1.92 \mathrm{eV}$. This may give rise to the low intensity of the slow component and the absence of clear bimodal feature at incidence energy of $0.99 \mathrm{eV}$.

\subsubsection{H atoms scattering from epitaxial graphene as a function of surface temperature}

In general, high temperatures increase the chance for reactants to cross the reaction barrier. Figure 6.7 shows results of $\mathrm{H}$ atoms scattering from epitaxial graphene at surface temperatures of $300 \mathrm{~K}$ and $600 \mathrm{~K}$. The incidence energy and angle are $1.92 \mathrm{eV}$ and $50^{\circ}$ for both temperatures. When the surface temperature is increased from $300 \mathrm{~K}$ to $600 \mathrm{~K}$, the intensity of the slow component increases and the intensity of the fast component decreases, indicating that more $\mathrm{H}$ atoms cross the barrier at higher temperature. It can be seen that the peak position and the width of the slow component is not altered by the increased surface temperature, as is shown more clearly in Figure 6.8. This indicates that increasing the surface temperature increases the chance of the $\mathrm{H}$ atom crossing the barrier, but does not change the energy transfer mechanism. The fast component seems to have a higher energy loss and a broader energy width at $900 \mathrm{~K}$, which is probably due to the overlap with the slow component.

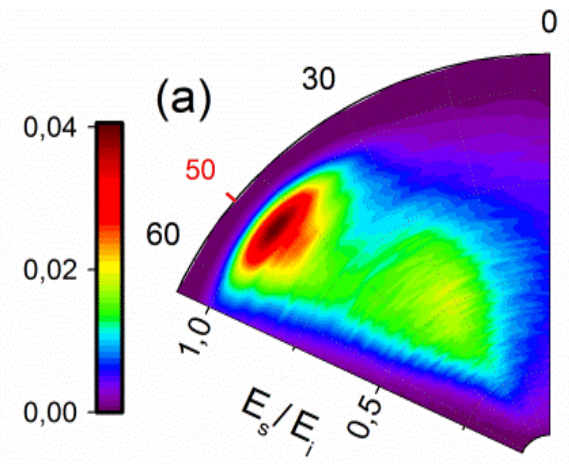

$\mathrm{T}=300 \mathrm{~K}$

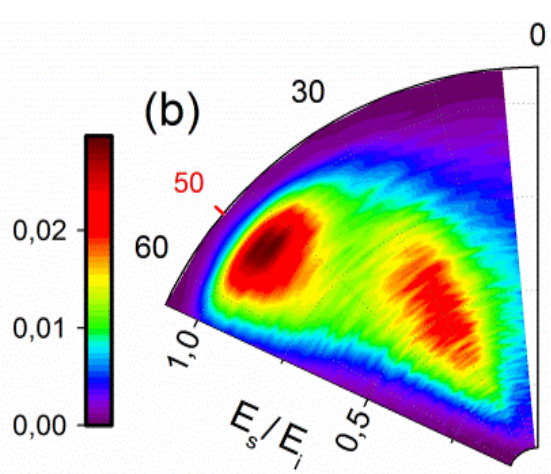

$\mathrm{T}=600 \mathrm{~K}$;

Figure 6.7: 2D translational energy and scattering angle distribution of $H$ atoms scattered from epitaxial graphene at surface temperatures of $300 \mathrm{~K}(\mathrm{a})$ and $600 \mathrm{~K}(\mathrm{~b})$. The incidence energy and angle are $1.92 \mathrm{eV}$ and $50^{\circ}$. 


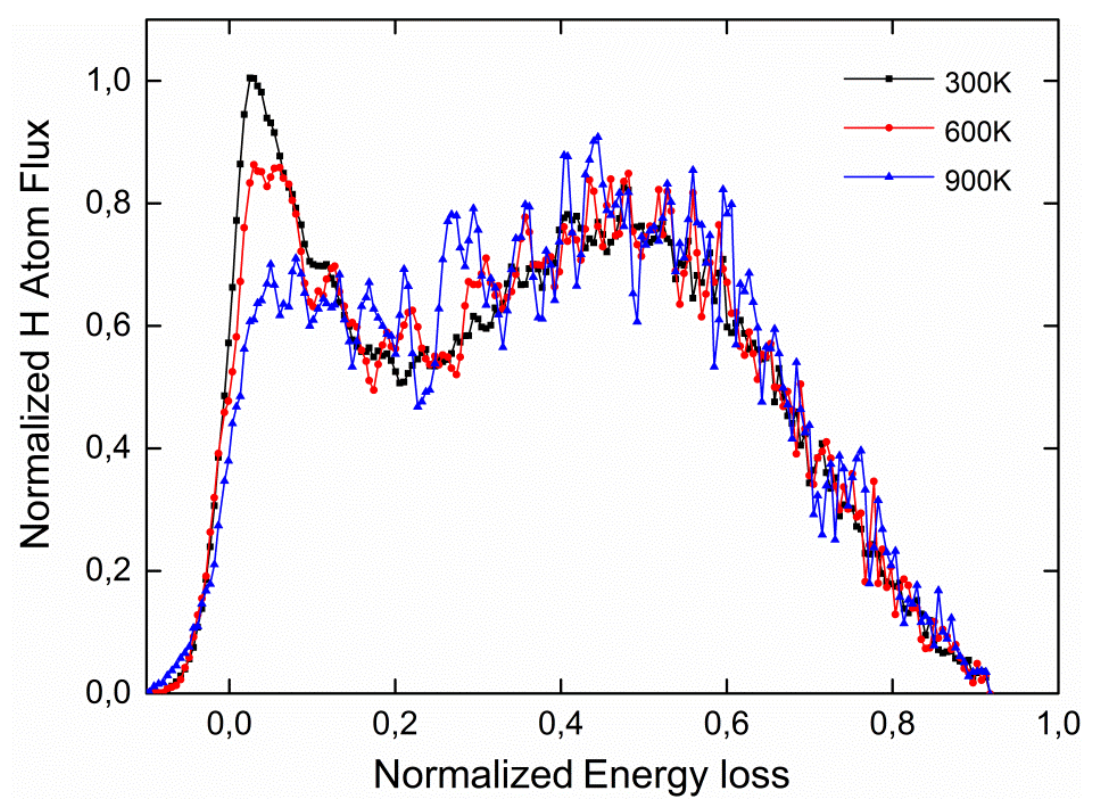

Figure 6.8: Normalized translational energy distribution of $H$ atoms scattering from epitaxial graphene at three different surface temperatures. The incidence and scattering angle are $50^{\circ}$ and $40^{\circ}$ respectively. The incidence energy is $1.92 \mathrm{eV}$.

\subsubsection{D atoms scattering on epitaxial graphene on various incidence conditions}

In this subchapter, the isotope effect of hydrogen atom scattering on epitaxial graphene is studied. Figure 6.9 shows the 2D translational energy and scattering angle distributions of D atoms scattering from epitaxial graphene at different incidence energies but similar incidence angles. The incidence energies are $0.94 \mathrm{eV}, 1.87 \mathrm{eV}$ and $3.25 \mathrm{eV}$ for panel (a) - (c). The incidence D atom beam is generated by photolysis of DI molecule. The incidence angles are $47.5^{\circ}, 48^{\circ}$ and $49.1^{\circ}$ for panel (a) - (c). The $\mathrm{D}$ atom scattering shows the same behavior as the $\mathrm{H}$ atom scattering. For scattering at incidence energy of $0.94 \mathrm{eV}$ and incidence angle of $47.5^{\circ}$, only the fast component is observed, indicating that no $\mathrm{D}$ atom passes the adsorption barrier. At higher incidence energy of $1.87 \mathrm{eV}$, the distribution shows a bimodal feature, indicating some $\mathrm{D}$ atoms cross the adsorption barrier. As the incidence energy is increased above $3.25 \mathrm{eV}$, there is only a slow component, indicating that all the $\mathrm{D}$ atoms have sufficient normal energy to cross the barrier. Figure 6.10 shows D atoms scattering from epitaxial graphene at different incidence angles but the same incidence energy. The incidence energy is $1.87 \mathrm{eV}$. Figure 6.10 looks similar to Figure 6.6, indicating the same dynamics. The fast and slow components of the scattered D 
atoms both shift to higher energy losses, and have broader FWHM in energy. This is as expected due to the mass difference of $\mathrm{H}$ and $\mathrm{D}$ atom.

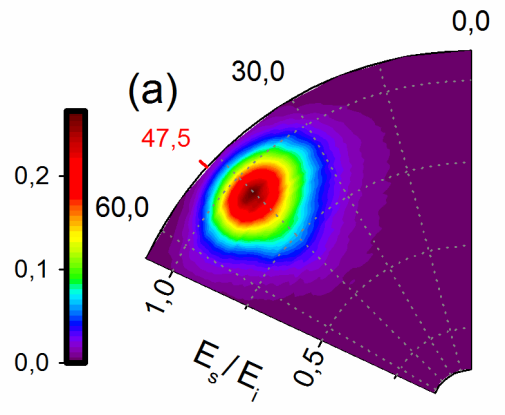

$E_{i}=0,94 \mathrm{eV}$

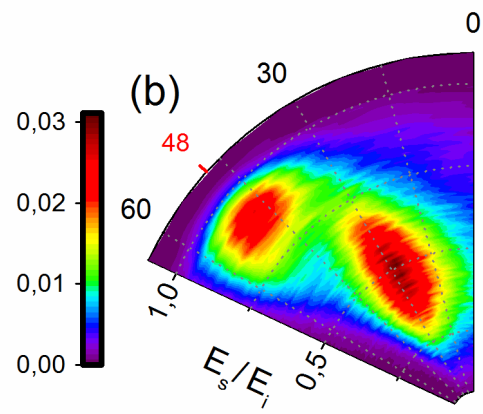

$E_{i}=1,87 \mathrm{eV} ;$

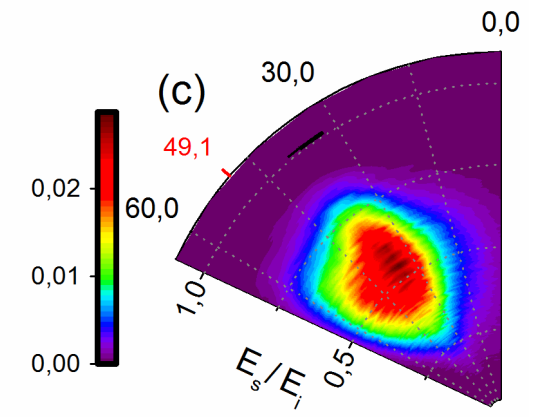

$\mathrm{E}_{\mathrm{i}}=3,25 \mathrm{eV} ;$

Figure 6.9: 2D translational energy and scattering angle distributions of D atoms scattered from epitaxial graphene. The incidence energies and specular scattering angles (red ticks) are indicated in each panel. 

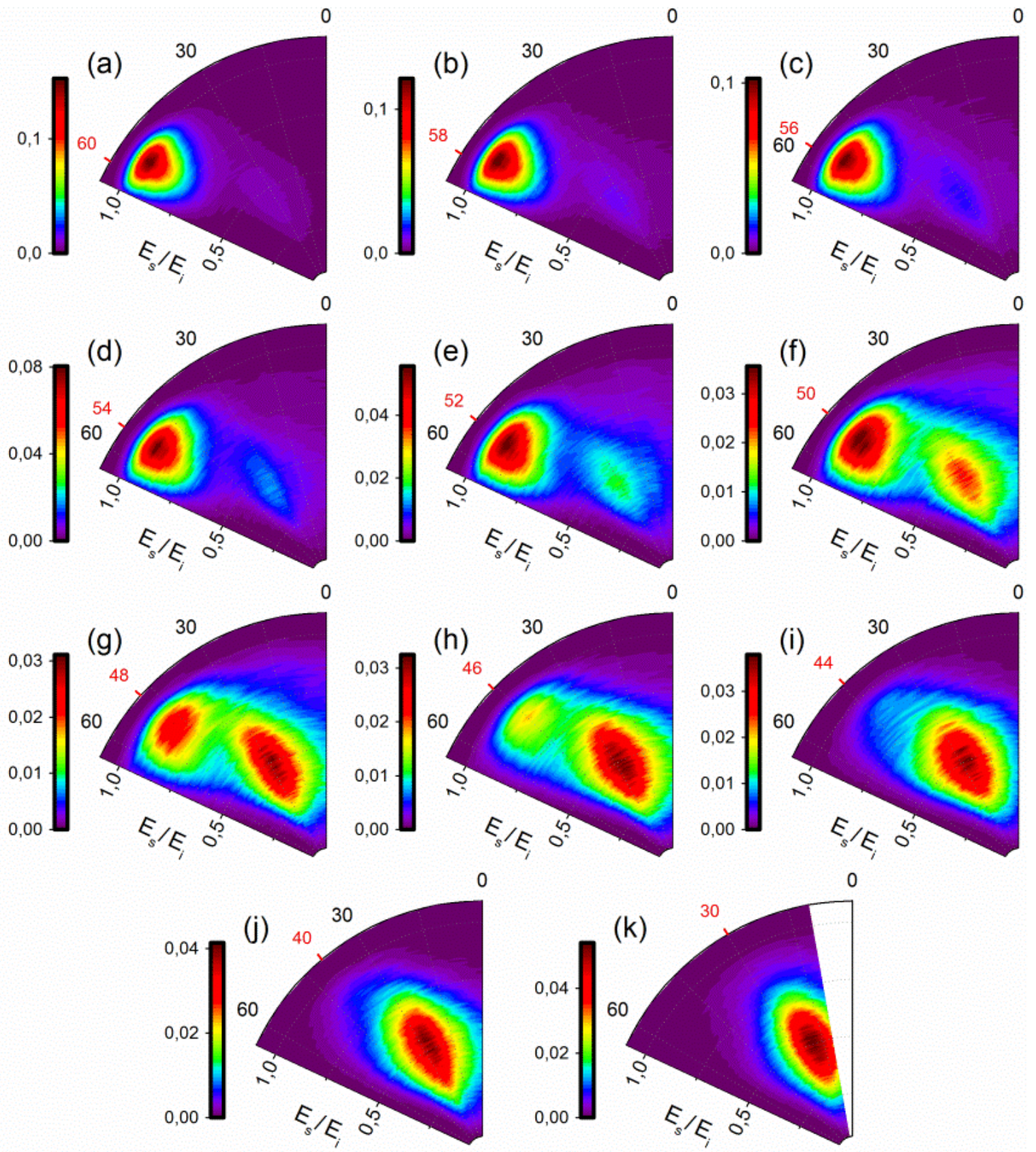

Figure 6.10: 2D translational energy and scattering angle distribution of $D$ atoms scattered from epitaxial graphene at different incidence angles but same incidence energy $(1.87 \mathrm{eV})$. The red ticks represent the specular scattering angle. 


\subsubsection{Summary of the experimental measurements}

In this section, I present the data for $\mathrm{H}$ and $\mathrm{D}$ atoms scattering on epitaxial graphene as a function of total incidence energy, incidence angle (normal incidence energy) and surface temperature, respectively. The 2D translational energy and scattering angle distributions exhibit a bimodal feature. The fast component is near-specular and near-elastic and exhibits narrow energy and angular distribution. The slow component has large energy loss (40\%-50\% of its initial translational energy) and is preferentially located around $30^{\circ}$ away from the surface normal. The ratio between the slow and fast component increases as increasing total incidence energy, normal incidence energy and surface temperature, respectively. This indicates the slow component originates from $\mathrm{H}$ atoms scattered back after crossing the adsorption barrier.

\subsection{Adsorption threshold of hydrogen atoms on epitaxial graphene on $\operatorname{Pt}(111)$}

In section 6.2, I conclude that hydrogen atoms scattered back from epitaxial graphene exhibit two separate components. The fast component originates from $\mathrm{H}$ atoms scattered back before crossing the adsorption barrier, and the other slow component can be assigned to the $\mathrm{H}$ atoms scattered back after passing the adsorption barrier. The ratio of the two components can be tuned by changing the normal incidence energy. Since the H/D atom flux is very low $\left(10^{8} \sim 10^{9}\right.$ atoms $/ \mathrm{cm}^{2} \cdot \mathrm{s}$ ), by monitoring the ratio of the two components, we can determine the zero coverage adsorption threshold (minimum normal incidence energy required for the hydrogen atoms to cross the adsorption barrier), above which the hydrogen atom could pass the adsorption barrier.

\subsubsection{Determining adsorption thresholds of $H / D$ atoms on epitaxial graphene}

The zero coverage adsorption threshold should be the normal incidence energy, at which the slow component appears. In this study I calculate the probability density peak-to-peak ratio of the slow component to the fast component to determine the adsorption threshold. When the ratio $\left(R=P_{s} / P_{f} ;\right)$ is larger than zero, there are some $\mathrm{H}$ atoms having enough normal energy to cross the adsorption barrier. The reason for calculating peak-to-peak ratio $R$ to determine the adsorption threshold is due to the experimental considerations. As explained in the introduction of Chapter 6, the probability density of every distribution is normalized to the integrated area (total scattering 
signal from all scattering angles). The total scattering signals are different for different incidence conditions. First, the incidence $\mathrm{H}$ atom beam flux may vary for different incidence conditions. Second, only the in-plane data can be recorded in the experiments, although a substantial amount of out-of-plane scattering is expected, based on the in-plane distribution. Hence calculating the peak-to-peak ratio $R$ avoids erroneous results induced by an analysis based on absolute numbers.

Figure 6.11 shows the peak-to-peak ratio $R$ plotted against normal incidence energies at different incidence conditions. All values shown in the figure are taken from the distributions which have clearly separated fast and slow components. Distributions that have overlapping fast and slow components are not used. Black squares represent $\mathrm{H}$ atom scattering at incidence energy of 1.92 $\mathrm{eV}$. The normal incidence energy varies from $0.48 \mathrm{eV}$ to $0.86 \mathrm{eV}$ (incidence angle from $60^{\circ}$ to $\left.48^{\circ}\right)$. For scattering at incidence energy of $1.92 \mathrm{eV}$, we can't further decrease the normal incidence energy, since we can't measure at incidence angles larger than $60^{\circ}$. Blue squares represent $\mathrm{H}$ atoms scattering at incidence energy of $0.99 \mathrm{eV}$, with normal incidence energy of $0.41 \mathrm{eV}$ (incidence angle of $50^{\circ}$ ). For scattering at incidence energy of $0.99 \mathrm{eV}$, we don't have well separated fast and slow component, so we can only determine the zero ratio value, at which there is only the fast component. As shown in figure 6.11, the zero coverage adsorption threshold appears to be in the range of $0.41 \mathrm{eV}$ to $0.48 \mathrm{eV}$ for $\mathrm{H}$ atoms. Red and pink circles represent $\mathrm{D}$ atoms scattering at incidence energy of $1.87 \mathrm{eV}$ and $0.94 \mathrm{eV}$, respectively. The incidence angle for the red circles ranges from $60^{\circ}$ to $48^{\circ}$ for the $1.87 \mathrm{eV}$ atoms, and is $47.5^{\circ}$ for the $0.94 \mathrm{eV}$ atoms. The measured zero coverage adsorption threshold for the $\mathrm{D}$ atoms is in the range of 0.43 $\mathrm{eV}$ and $0.47 \mathrm{eV}$. Here we note the experimentally determined ranges are simply intervals of last two data points, it might be narrowed by further measurements with smaller intervals. 


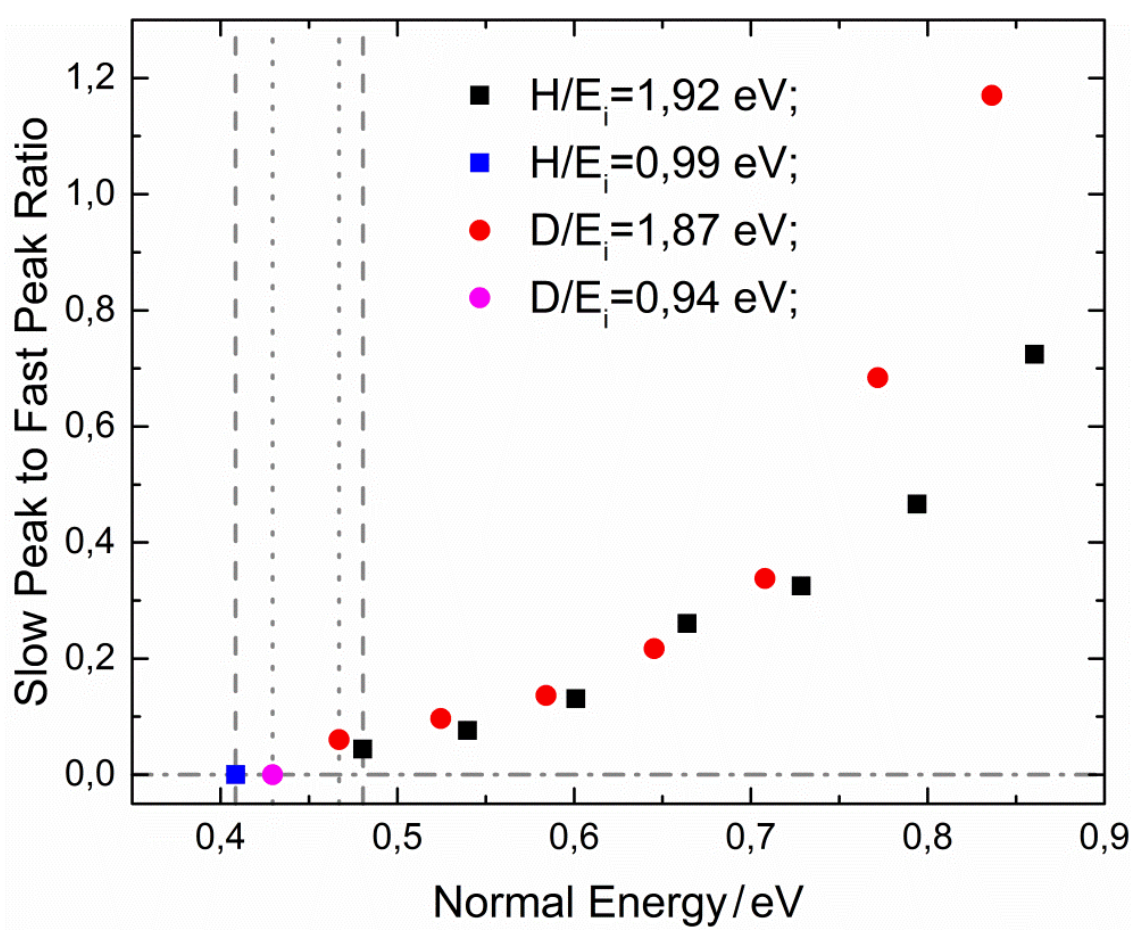

Figure 6.11: Intensity ratio of the slow peak to the fast peak for $H$ and $D$ atoms scattering from epitaxial graphene at different normal incidence energies. Squares represent data from $H$ atom scattering and circles represent data from D atom scattering. The incidence energies are $1.92 \mathrm{eV}$ and $0.99 \mathrm{eV}$ for $\mathrm{H}$, and $1.87 \mathrm{eV}$ and $0.94 \mathrm{eV}$ for $D$. Gray dashed lines indicate the range of the $\mathrm{H}$ atom zero coverage adsorption threshold. Gray dotted lines indicate the range of the D atom zero coverage adsorption threshold.

\subsubsection{A model for fitting the adsorption threshold}

In section 6.3.1, we use the slow peak to fast peak ratio $\left(R=P_{s} / P_{f} ;\right)$ to determine a range of zero coverage adsorption threshold for the hydrogen atom on epitaxial graphene. In this chapter, a model for the experimental findings is presented. The model is based on several physically reasonable assumptions as discussed below.

1. The fate of the incidence hydrogen atoms is categorized into three channels: scattered back without crossing the adsorption barrier $\left(\mathcal{P}_{F}\right)$, scattered back after crossing the adsorption barrier $\left(\mathcal{P}_{S}\right)$, or become adsorbed after crossing the adsorption barrier $\left(\mathcal{P}_{A}\right)$. The probabilities for the three channels add up to one: 


$$
\mathcal{P}_{F}+\mathcal{P}_{S}+\mathcal{P}_{A}=1
$$

Assume the probabilities $\mathcal{P}_{F}$ and $\mathcal{P}_{S}$ are proportional to their peak values $P_{f}$ and $P_{s}$, respectively. Then Eq. 6.1 is written as:

$$
a P_{f}+b P_{s}+\mathcal{P}_{A}=1
$$

Here we also assume that the parameters $a$ and $b$ are constant for different incidence angles but the same incidence energy.

2. For the same incidence energy, incidence atom species, and surface temperature, the adsorption probability $\mathcal{P}_{A}$ is proportional to the probability of scattering back after crossing the adsorption barrier $\mathcal{P}_{S}$. This assumption is based on the fact that the average energy loss of the $\mathrm{H}$ atoms scattered back after crossing the barrier is only dependent on the incidence energy, but not on the incidence angle, indicating a strong mix between energies parallel and perpendicular to the surface. It will be shown in detail in chapter 6.4. The adsorption probability $\mathcal{P}_{A}$ is then written as:

$$
\mathcal{P}_{A}=C \times \mathcal{P}_{S}=C \times b P_{S}=c P_{S}
$$

where $c=C \times b$. This changes Eq. 6.1 to:

$$
A P_{f}+B P_{s}=1 \text { or } P_{f}=1 / A-B / A P_{S}
$$

where $A=a$ and $\mathrm{B}=b+c$. The parameters $\mathrm{A}$ and $\mathrm{B}$ can be calculated by fitting $P_{f}$ and $P_{s}$ to a straight line.

3. The peak intensity of the slow component $P_{s}$ is only a function of normal incidence energy $P_{s}=f\left(E_{n}\right)$, when other incidence conditions (incidence energy, surface temperature and incidence species) are fixed. Here we further assume the function has an empirical form,

$$
P_{s}=f\left(E_{n}\right)= \begin{cases}0, & E_{n} \leq E_{0} \\ m\left(E_{n}-E_{0}\right)^{n}, & E_{n}>E_{0}\end{cases}
$$

Parameters $m$ and $n$ depends on the total incidence energy, incidence atom species and surface temperatures. Parameter $E_{0}$ represents the adsorption threshold, which will be clear in Eq. 6.6. After some rearrangement, we derive an empirical expression for the peak-to-peak ratio as: 


$$
R=\frac{P_{s}}{P_{f}}= \begin{cases}0, & E_{n} \leq E_{0} \\ k\left(1+\frac{t}{\left(E_{n}-E_{0}\right)^{n}-t}\right), & E_{n}>E_{0}\end{cases}
$$

The parameter $k=-B / A$ is the slope of Eq. 6.4, and the parameter $t=1 / A m$. When $E_{n}$ equals parameter $E_{0}, R$ equals 0 . This indicates that $E_{0}$ is the adsorption threshold.

Before showing the fitting results, I will first discuss the meaning of the fitting function $f$ in Eq. 6.5. Based on the three assumptions made above, the physical meaning of the fitting function $f$ in Eq. 6.5 is that it is proportional to the probability for a hydrogen atom to cross the adsorption barrier under certain incidence conditions. $f$ depends on all the incidence conditions including incidence energy, incidence angle, surface temperature, and incidence atom species. When other incidence conditions are fixed, it is then only a function of the normal incidence energy. In principle, $f$ can be generated from dynamical calculations based on the multi-dimensional PES of hydrogen atoms on graphene. Unfortunately, this kind of calculation does not exist, owing to the absence of a multi-dimensional PES. As a result, assumptions have to be made on the form of $f$.

As shown in Figure 6.11, the peak-to-peak ratio $R$ should be 0 below the adsorption threshold, and goes to infinity above certain normal incidence energy where the fast component no longer contributes. Mathematically, an empirical form shown in Eq. 6.5 fulfills both conditions. The physical picture of this empirical form is not as obvious as the mathematical one. A simple model assumption can be used to understand the power function form of $f$. The adsorption transition state of hydrogen atom on graphene is above the top site (39). The PES has cylindrical symmetry around the top site (it is actually three-fold symmetry, but cylindrical symmetry is a good approximation). Here we omit the dynamical steering effect and movements of the $\mathrm{C}$ atoms, and assume the $\mathrm{H}$ atom with normal energy $E_{n}$ can cross the adsorption barrier if the intersection of the initial trajectory with the surface is within a certain distance $\rho$ (impact parameter) away from the top site. The probability of a single $\mathrm{H}$ atom crossing the barrier is then proportional to the area $S=\pi \rho^{2}$ (adsorption cross section). If the potential energies near the transition state have a form of $E(\rho)-E_{0} \propto \rho^{2}$, then the function $f$ would have the form $f \propto P_{s} \propto \pi \rho^{2} \propto\left(E_{n}-\right.$ $\left.E_{0}\right)$. If the potential energies near the transition state have a form of $E(\rho)-E_{0} \propto \rho^{4}$, then the function $f$ would have the form $f \propto P_{S} \propto \pi \rho^{2} \propto\left(E_{n}-E_{0}\right)^{1 / 2}$. It should be noticed that this simple model assumption only helps to give a qualitative pictorial understanding of the function $f$. 
In the real world, dynamical steering effects and the $\mathrm{C}$ atoms movements strongly affect the form of $f$.

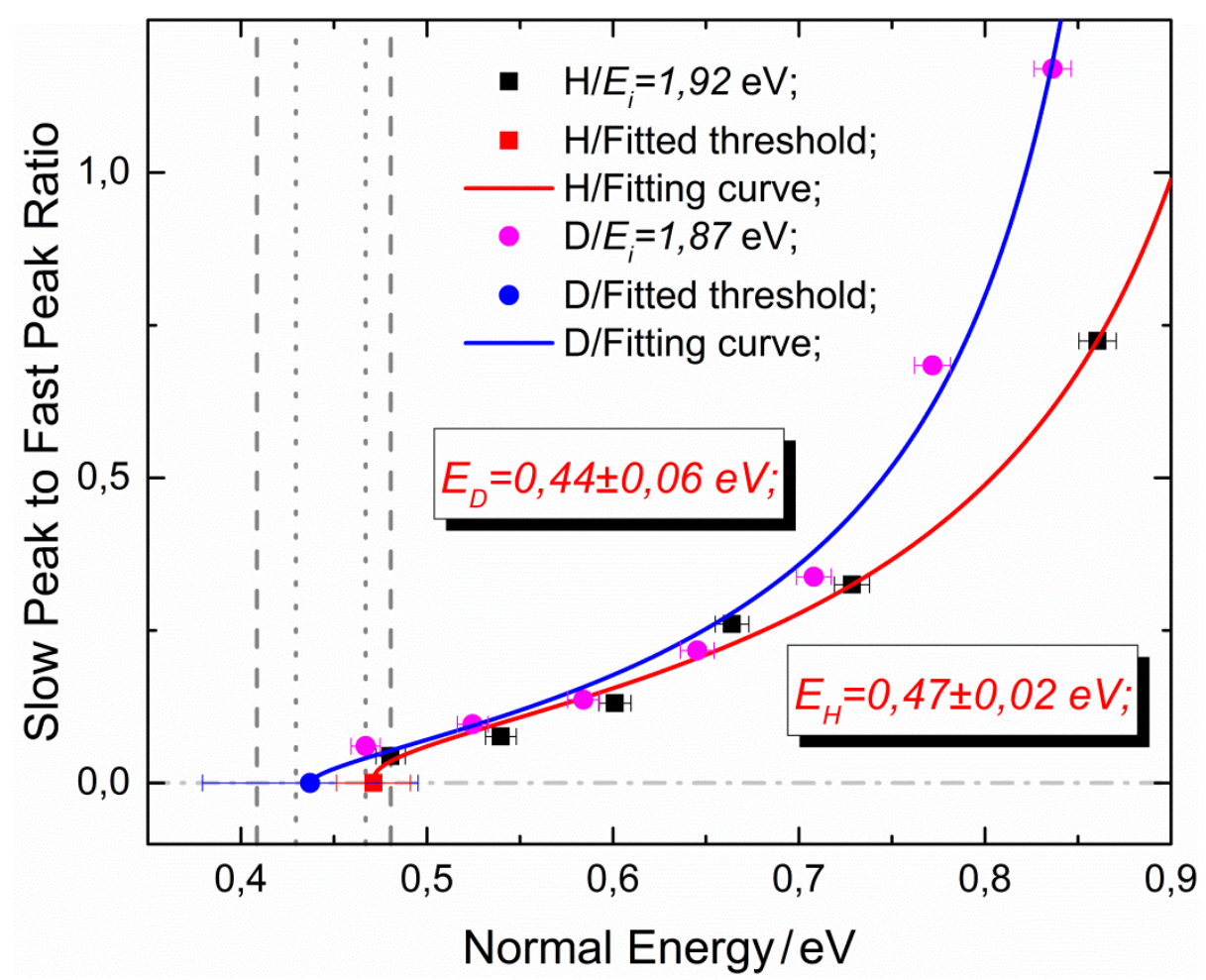

Figure 6.12: The best fitting of the peak-to-peak ratio to Eq. 6.6. Gray dashed lines represent the range of the $H$ atom zero coverage adsorption threshold determined by the experimental data. Gray dotted lines represent the range of the $D$ atom zero coverage adsorption threshold determined by the experimental data.

Figure 6.12 shows the fitting of the measured ratio to Eq. 6.6. Since the fitting should be restricted to the same incidence energy (parameters $m$ and $n$ are then constant), only data points from $1.92 \mathrm{eV} \mathrm{H}$ atoms scattering and $1.87 \mathrm{eV} \mathrm{D}$ atoms scattering are used. The error bar in the normal energy is due to the beam energy width $( \pm 10 \mathrm{meV})$ and incidence angle uncertainty $\left( \pm 0.1^{\circ}\right)$. The time-of-flight spectra were averaged over a long period (typically 1800-2700 shots for both signal and background measurements), so we omit the error bar for the peak-to-peak ratio. An orthogonal distance regression algorithm is used to fit the data to the minimum residual sum of squares. The goodness of the fit is indicated by the small residual sum of square (RSS) and the adjusted R square (Adj. R-Square) close to 1 . The best fit of the zero coverage adsorption threshold is $0.47 \pm 0.02 \mathrm{eV}$ for $\mathrm{H}$, and $0.44 \pm 0.06 \mathrm{eV}$ for $\mathrm{D}$. The fitted parameters are listed in table 
6.1. Here we note that parameters $k, t$ and $n$ are quite different for $\mathrm{H}$ and $\mathrm{D}$. The difference in $k$ may come from the different widths of the distributions for $\mathrm{H}$ and $\mathrm{D}$. The differences in $t$ and $n$ may be due to the different adsorption thresholds for $\mathrm{H}$ and $\mathrm{D}$, which cause different barrier crossing probability for the same normal incidence energy.

\begin{tabular}{|c|c|c|}
\hline & H & D \\
\hline $\boldsymbol{k}$ & $-0.08688 \pm 0.00706$ & $-0.147 \pm 0.02078$ \\
\hline $\boldsymbol{t}$ & $0.84438 \pm 0.02464$ & $0.68281 \pm 0.03415$ \\
\hline $\boldsymbol{n}$ & $0.29903 \pm 0.03954$ & $0.54294 \pm 0.1209$ \\
\hline $\boldsymbol{E}_{\boldsymbol{0}}$ & $0.47127 \pm 0.01988$ & $0.43728 \pm 0.05795$ \\
\hline $\boldsymbol{R S S}$ & $7.6352 \times 10^{-4}$ & 0.0076 \\
\hline Adj. R-Square & 1 & 0.9999 \\
\hline
\end{tabular}

Table 6.1: Parameters for the best fits shown in Figure 6.13.

\subsubsection{Discussions on the adsorption threshold}

In this section, we will discuss some issues related to the zero coverage adsorption threshold of the H/D atom on epitaxial graphene on $\mathrm{Pt}(111)$. These issues include the fitting function, differences between the adsorption threshold and the adsorption barrier, the role of the $\operatorname{Pt}(111)$ substrate, the isotope effect of the adsorption threshold, and comparison to the theoretically predicted adsorption barrier heights.

Discussion on the fitting function: It should be noted that the fitting function is empirical. The value of the fitted adsorption threshold strongly depends on the form of $f$. For example if we use $m\left(E_{n}-E_{0}\right)$ instead of $m\left(E_{n}-E_{0}\right)^{n}$ in the fitting for $\mathrm{D}$ atom, the fitted adsorption threshold will be $0.3 \pm 0.07 \mathrm{eV}$. But the $R S S$ will be two times as large and the fitted threshold is outside the experimentally determined range. If we use $m\left(E_{n}-E_{0}\right)$ in the fitting for $\mathrm{H}$ atom, we will get a threshold value smaller than 0 , which is non-physical. In the absence of high level theoretical description of $f$, we cannot further explain why $f=m\left(E_{n}-E_{0}\right)^{n}$ gives such a good fitted adsorption threshold value.

It is interesting that there is an inflection point near the vicinity of the fitted adsorption threshold. This is purely due to the used form of $f$. When the power parameter $n$ is smaller than one, the 
inflection point always exists. The reason for the inflection point is similar to that in a tangential function, and is purely mathematical.

\section{Difference between the adsorption barrier height and the measured adsorption threshold:}

The adsorption barrier height is commonly defined in the theoretical literatures as the potential energy difference between the adsorption transition state and the state at which graphene and $\mathrm{H}$ atoms no longer interact. The adsorption barrier height is usually calculated at static atomic positions, and is comprised of two main parts. One part of the adsorption barrier height comes from the reconstruction energy for graphene. As shown in chapter 5.3, the $\mathrm{C}$ atom has to pucker up about $0.1 \AA$ to reach the adsorption transition state, increasing the potential energy of the system. This energy can be estimated to be $70 \mathrm{meV}$ at a harmonic force constant of $14.3 \mathrm{eV} / \AA^{2}$ $(90,109)$ and a displacement of $0.1 \AA(106)$. The second part of the adsorption barrier height comes from the energy for localizing electrons for the $\mathrm{C}-\mathrm{H}$ bond formation (112). The measured adsorption threshold from our experiments differs from the adsorption barrier heights given in theoretical literatures. First, the measured threshold is purely for hydrogen atom translational energy. It does not contain the reconstruction energy for the graphene, since the interaction of the $\mathrm{H}$ atom and graphene is repulsive before crossing the barrier. Second, the measured threshold contains zero point energy (ZPE), which is usually not included in potential energy surface calculations. Third, the measured adsorption threshold corresponds to a dynamical adsorption barrier but not the static adsorption barrier, since the $\mathrm{C}$ atom may not have the chance to pucker up to the transition state configuration during the scattering process. Fourth, quantum tunneling effects need to be considered. Atoms whose normal energies are below the barrier height can tunnel through the barrier. When comparing the measured adsorption threshold to theoretically calculated barrier heights, these considerations must be taken into account.

Role of the $\operatorname{Pt}(111)$ substrate: In general, $\mathrm{H}$ atom adsorption on epitaxial graphene on $\mathrm{Pt}(111)$ should have a lower barrier than adsorption on pristine graphene. Hydrogen atom adsorption on epitaxial graphene on $\mathrm{Pt}(111)$ should have a stronger $\mathrm{C}-\mathrm{H}$ bond than adsorption on pristine graphene, due to the stabilization of the neighbor C-Pt bond. Simply based on Bell-EvansPolanyi rule, a stronger $\mathrm{C}-\mathrm{H}$ bond indicates a smaller adsorption barrier height. Several reasons could cause the energetic difference between adsorption on pristine and epitaxial graphene. First, epitaxial graphene on $\operatorname{Pt}(111)$ has curvatures, and the pre-puckering would reduce the adsorption 
barrier (136). Second, epitaxial graphene on Pt(111) is slightly hole doped (less than $-0.001 \mathrm{e} / \AA^{3}$ (94)), and the dipole formed between the graphene and the $\operatorname{Pt}(111)$ will reduce the adsorption barrier.

So far there has been no quantitative study on the barrier height difference between adsorption on epitaxial graphene on $\operatorname{Pt}(111)$ and pristine graphene. In chapter 5.2, we already discussed the interaction of the $\operatorname{Pt}(111)$ substrate and the epitaxial graphene, which is one of the weakest among all the metal substrates (the hole doping is less than $-0.001 \mathrm{e} / \AA^{3}$ ). The graphene phonon structure and electronic structure are barely affected by the $\operatorname{Pt}(111)$ substrate (97). The distance between the epitaxial graphene and $\operatorname{Pt}(111)$ substrate is around $3.3 \AA$, which is close to the distance between graphene layers in graphite (89). The graphene reconstruction at the transition state configuration for the zero coverage adsorption event is small (only about $0.1 \AA$ for the top $\mathrm{C}$ atom), it is not expected to change the interaction of graphene and $\mathrm{Pt}(111)$ substrate very much. Based on these reasons, we speculate the barrier height difference between adsorption on pristine graphene and epitaxial graphene on $\operatorname{Pt}(111)$ should be small for the very first adsorption event. However, for adsorptions at high coverage considered in other studies, the difference should be more significant.

Isotope effect of the adsorption threshold: As shown In Figure 6.13, there is a minor isotope effect on adsorption of a hydrogen atom on epitaxial graphene. Electronic structures for $\mathrm{H}$ and D atom adsorption on graphene should be the same. However, several factors could introduce differences in the adsorption. First, the ZPE would cause a higher adsorption threshold for the $\mathrm{H}$ atom. Second, the dynamical barrier effect should also be stronger for H. Since the H atom moves faster than the $\mathrm{D}$ atom at the same energy, the $\mathrm{C}$ atom has more time to adjust its movement during the scattering of the $\mathrm{D}$ atom. Third, the quantum tunneling effect would cause a lower adsorption threshold for the $\mathrm{H}$ atom.

These three effects cannot be separated in our measurements, and their magnitudes can only be estimated. Theoretical calculation predicts a ZPE of $0.043 \mathrm{eV}$ for $\mathrm{H}$ atom adsorption and 0.021 $\mathrm{eV}$ for $\mathrm{D}$ atom adsorption (128). The overall ZPE difference between $\mathrm{H}$ and $\mathrm{D}$ is then about 0.02 $\mathrm{eV}$. Predictions on the dynamical barrier effect and the quantum tunneling strongly depend on the detailed shape of the multi-dimensional PES and the incidence conditions. There has been no theoretical prediction on these two effects. As shown in figure 6.13, the experimentally 
determined ranges of the zero coverage adsorption threshold for $\mathrm{H}$ and $\mathrm{D}$ atom overlap with each other. The best fitted adsorption threshold for $\mathrm{H}$ atom is $0.03 \mathrm{eV}$ higher than that for $\mathrm{D}$ atom; however the best fitted values for $\mathrm{H}$ and $\mathrm{D}$ also have overlapping error bars. It is hard to quantitatively determine how large the quantum tunneling and dynamical barrier effects are based on our measurement, but the small difference in the adsorption thresholds for $\mathrm{H}$ and $\mathrm{D}$ atom indicates that the effects are minor. Their minor effects can be understood as follows. Quantum tunneling is usually profound at low temperatures and low incidence energies (137). At the temperatures and incidence energies used in the experiments in this thesis, quantum tunneling should be small enough to be neglected. The minor dynamical barrier effect could be due to the pre-puckering of the $\mathrm{C}$ atoms. At room temperature, the average displacement of $\mathrm{C}$ atoms from its equilibrium position is $0.065 \AA$. The Moiré structure also introduces some pre-puckering of the $\mathrm{C}$ atoms (84). There should be a certain number of $\mathrm{C}$ atoms that pre-puckers to the position for the adsorption transition state ( $0.1 \AA ̊$ above the graphene sample) for the scattering.

The peak-to-peak ratio for $\mathrm{H}$ and $\mathrm{D}$ also exhibits large differences. Several factors give rise to the differences. First, in figure 6.6 and 6.11 , it can be clearly seen that the width of the distributions of fast and slow component is different for the $\mathrm{H}$ and $\mathrm{D}$ atom. This gives rise to different absolute peak values. Second, the $\mathrm{H}$ and $\mathrm{D}$ atom should experience a steering effect of different magnitude during the scattering process, due to the mass difference. This means the barrier crossing probabilities are different for $\mathrm{H}$ and $\mathrm{D}$ atoms at the same normal incidence energy.

Comparison to the theoretically predicted adsorption barrier heights: There have been a vast number of energetic calculations involving single hydrogen atom adsorption on graphene. They can be divided into four categories. The first category includes calculations based on LSDA or GGA level DFT calculations. Graphene slabs with several unit cells and plane wave basis sets are usually used in these calculations. Most of the existing calculations belong to this category. The calculated values depend on the employed functional and the graphene slab size. The calculated adsorption barrier heights range from $0.15 \mathrm{eV}$ to $0.25 \mathrm{eV}(106,107,109,110,112$, 132). The second category includes Van der Waals corrected DFT calculation. Typically, Van der Waals correction reduces the barrier height. This category gives a barrier height around 0.1 $\mathrm{eV}$ (120). The third category is also comprised of DFT calculations, but uses aromatic molecules 
to simulate graphene. Results of this category depend on the molecular species, employed functional and basis set. The calculated barrier heights vary between $0.1 \mathrm{eV}$ and $0.3 \mathrm{eV}(39,104)$. The last category is based on more accurate wave function methods. Due to the difficulties in adapting periodical boundary conditions in wave function methods, aromatic molecules are usually used to simulate graphene. A recent basis set superposition error corrected ROCCSD(T) calculation predicted a barrier height of $0.39 \mathrm{eV}$ for $\mathrm{H}$ atom adsorption on Pyrene, and a barrier height of $0.40 \mathrm{eV}$ for $\mathrm{H}$ atom adsorption on Coronene. Adding ZPE, the barrier height is $0.44 \mathrm{eV}$ for $\mathrm{H}$ atom adsorption on Coronene (39). This is already within our experimentally determined range of zero coverage adsorption threshold.

\begin{tabular}{|c|c|c|}
\hline Method & System & Barrier \\
\hline DFT/PW91 ${ }^{(111)}$ & $\mathrm{H}+$ Graphene $(3 \times 3)$ & $0,24 \mathrm{eV}$ \\
\hline DFT/PBE ${ }^{(112)}$ & $\mathrm{H}+$ Graphene $(5 \times 5)$ & $0,20 \mathrm{eV}$ \\
\hline DFT/PBE-D3 ${ }^{(120)}$ & $\mathrm{H}+$ Graphene $(3 \times 3)$ & $0,13 \mathrm{eV}$ \\
\hline DFT/B3LYP ${ }^{(39)}$ & $\mathrm{H}+$ Coronene & $0,34 \mathrm{eV}$ \\
\hline $\operatorname{ROCCSD}(T)^{(39)}$ & $\mathrm{H}+$ Coronene & $0,40 \mathrm{eV}$ \\
\hline $\operatorname{ROCCSD}(\mathrm{T})+\mathrm{ZPE}{ }^{(39)}$ & $\mathrm{H}+$ Coronene & $0,44 \mathrm{eV}$ \\
\hline
\end{tabular}

Table 6.2: Adsorption barriers predicted by different theoretical simulations.

Several aspects have to be considered when a comparison of our experimentally determined threshold to the theoretical barrier height is made. The experimentally determined threshold does not include the graphene reconstruction energy and could be influenced by dynamical barrier effects, quantum tunneling and the $\operatorname{Pt}(111)$ substrate. Based on the discussions in earlier sections, it is reasonable to neglect the influences from the dynamical barrier effect, quantum tunneling and the Pt(111) substrate. Adding reconstruction energy (+70 meV) and the ZPE (-43 meV and $21 \mathrm{meV}$ for $\mathrm{H}$ and $\mathrm{D})$ to the experimentally determined zero coverage adsorption threshold, we predict the adsorption barrier height to be in the range of $0.44 \mathrm{eV}$ to $0.51 \mathrm{eV}$ for $\mathrm{H}$ atom, and $0.48 \mathrm{eV}$ to $0.52 \mathrm{eV}$ for $\mathrm{D}$ atom. The best fitted adsorption barrier height is $0.50 \pm 0.02 \mathrm{eV}$ for $\mathrm{H}$ atom, and $0.49 \pm 0.06 \mathrm{eV}$ for $\mathrm{D}$ atom. Apparently, the $\mathrm{CC}$ calculations give much better agreement with the experimental results than DFT calculations. 


\subsection{Dynamics of hydrogen atoms scattering from epitaxial graphene on $\operatorname{Pt}(111)$ : Two different scattering channels}

In last two sub-sections, it is clearly seen that the scattering of hydrogen atoms from epitaxial graphene involves two channels. The fast component originates from the scattering of $\mathrm{H}$ atoms before crossing the adsorption barrier. The slow component is assigned to the scattering of $\mathrm{H}$ atoms after crossing the adsorption barrier. The two components have different translational energy and scattering angle distributions. In this chapter, I will discuss the dynamics of the two scattering channels. Experimental results are compared to recent AIMD simulations from Marvin Kammler et al. (138). The simulations were performed using VASP 5.3 .5 (139-142). Projectoraugmented waves were used in combination with pseudopotentials to model the electron-ion interaction (143-145). The slab size was chosen as 3 graphene unit cells in $\mathrm{x}$-direction and 4 unit cells in $\mathrm{y}$-direction to minimize the interaction of the $\mathrm{H}$-atom with its images. The energy cutoff was chosen as $400 \mathrm{eV}$, the step-size was $0.1 \mathrm{fs}$, and the tetrahedron method with Blöchl corrections was used to treat partial occupancies with a smearing of $0.2 \mathrm{eV}$. This required a $8 \times 8 \times 1$ k-point mesh centered at the gamma point. All calculations were performed with the PBE functional and the D2 van der Waals correction. Spin-polarization was taken into account at all times. The global break condition for the electronic self-consistency-loop was reached when both the total free energy change and the band structure energy change were below $0.01 \mathrm{meV}$. A trajectory simulation was regarded as complete if either the H-atom reached its initial height above the surface or if the simulated time exceeded $200 \mathrm{fs}$ (138). The calculation gives an adsorption barrier of about $0.2 \mathrm{eV}$ and bond strength of $0.7 \mathrm{eV}$. The calculated $\mathrm{C}-\mathrm{H}$ bond length is $1.1 \AA$ and the $\mathrm{C}$ atom displacement is $0.4 \AA$. These results agree well with other DFT based calculations $(106,107,146)$. As shown in the previous section, DFT calculations usually predict a lower adsorption barrier height than experimental measurement. However, the computational efficiency of DFT still makes it the best choice for AIMD calculations.

\subsubsection{An overall comparison between the theoretical and experimental data}

Figure 6.13 shows the translational energy loss distributions of $\mathrm{H}$ atoms scattering from graphene, obtained from AIMD calculation and experimental measurement. The distributions are integrated over all scattering angles. The incidence energy is $1.92 \mathrm{eV}$. The incidence angles are $30^{\circ}$ and $48^{\circ}$ for the experimental results, and $34^{\circ}$ and $52^{\circ}$ for calculated results. The AIMD calculations have 
122 trajectories $\left(52^{\circ}\right)$ and 86 trajectories $\left(34^{\circ}\right)$. The incidence direction in the AIMD simulation is parallel to the plane defined by the $\mathrm{C}-\mathrm{C}$ bond and the surface normal. Since there is a difference between the experimentally derived adsorption barrier height and theoretically calculated one, the experimental incidence angles are chosen to give a better agreement on the translational energy loss distributions obtained from calculations and experiments. Qualitative agreement is observed in the integrated translational energy loss distributions. At large incidence angles, both theoretical calculation and experimental measurement exhibit the bimodal feature. For small incidence angles, both distributions only have the slow component, indicating all the $\mathrm{H}$ atoms have enough normal incidence energy to cross the barrier. The difference between simulations and experiments may arise from the small amount of simulation trajectories or from the inaccuracy of the DFT description on $\mathrm{H}$ atoms scattering on epitaxial graphene.

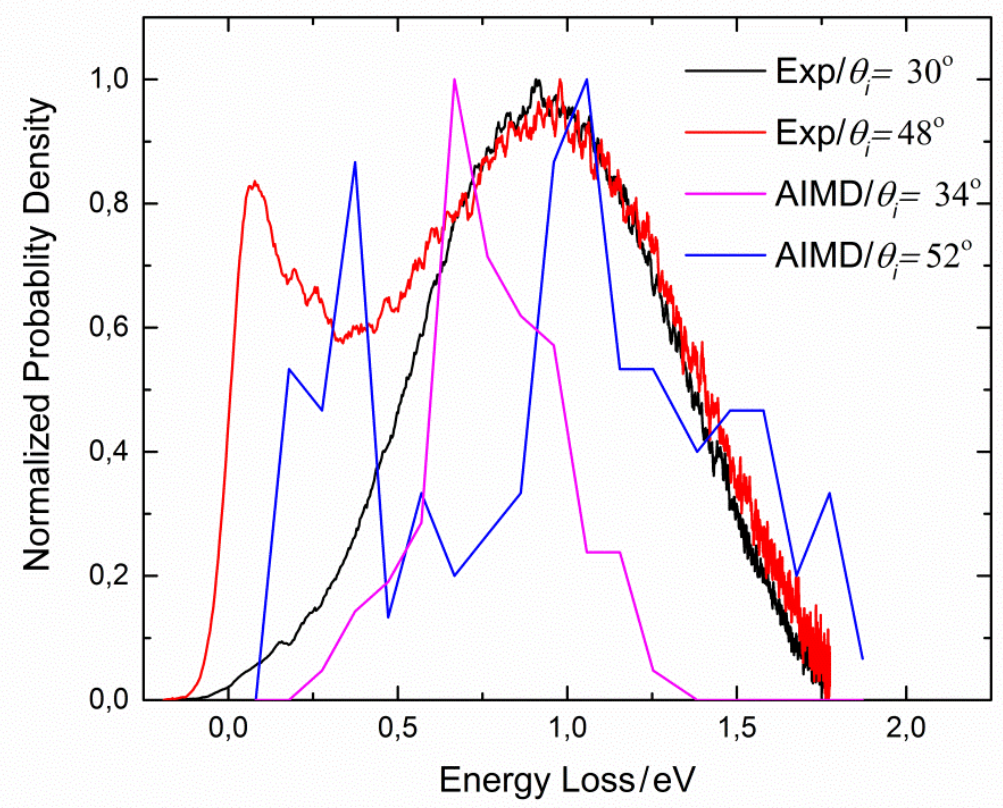

Figure 6.13: Normalized energy loss distribution of $H$ atoms scattering from graphene integrated over all measured scattering angles $\left(0^{\circ}-65^{\circ}\right)$. Incidence energy is $1.92 \mathrm{eV}$. Incidence angles are indicated in the figure. Blue and pink curves are from AIMD simulation (138). Black and red curves are from experimental data. AIMD calculations have 122 trajectories (blue) and 86 trajectories (magenta,) respectively. 
Figure 6.14 shows the 2D H atom translational energy loss and scattering angle distribution from AIMD calculation (upper panels) and experimental measurement (lower panels). Each point represents the outcome of one trajectory. Red points in the AIMD figures show the trajectories in which the $\mathrm{H}$ atoms cross the adsorption barrier, and green points represent the trajectories in which the $\mathrm{H}$ atoms do not cross the adsorption barrier.
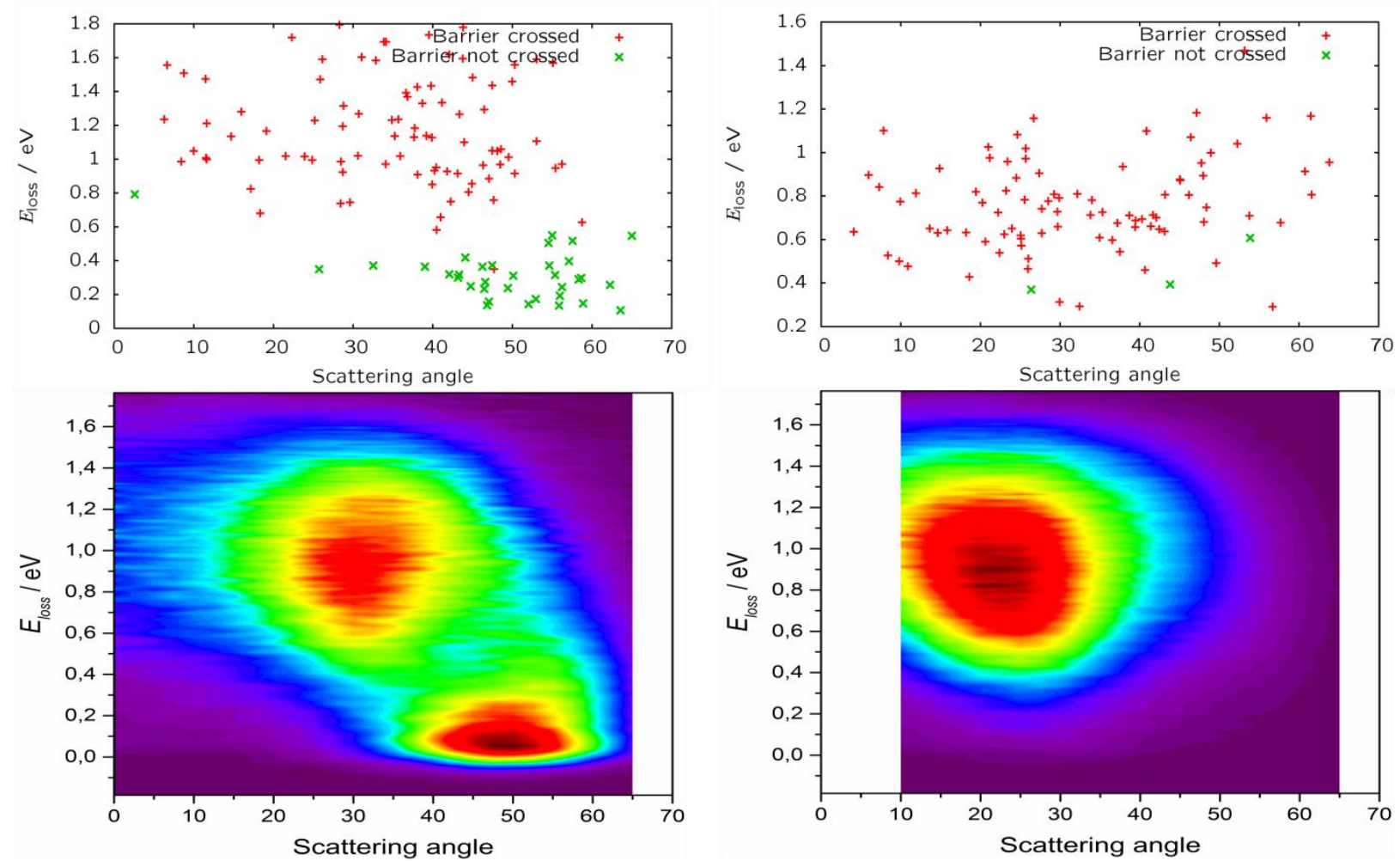

Figure 6.14: 2D H atom translational energy loss and scattering angle distribution from AIMD calculations (upper panels) and experimental measurements (lower panels). The incidence energies are all $1.92 \mathrm{eV}$. The incidence angles for AIMD calculations are $52^{\circ}$ (upper left) and $34^{\circ}$ (upper right) respectively. AIMD calculations have 122 trajectories (upper left) and 86 trajectories (upper right), respectively. Each point represents the outcome of one trajectory. The incidence angles for experimental measurements are $48^{\circ}$ (lower left) and $30^{\circ}$ (lower right), respectively (138).

Due to the small number of trajectories, quantitative comparisons between experimental and theoretical results are not possible. However, qualitatively the experimental and theoretical results agree well with each other. As shown in the upper and lower left panels of Figure 6.14, in 
both the AIMD simulations and experimental measurements, two components are observed, which are separated from one another due to the fact that one involves barrier crossing and the other does not. The fast and slow components in the simulation show similar energy and angular distributions to the two components in the experimental results. This agreement provides the theoretical support of our experimental conclusion that the separation of the two components in the translational energy and scattering angle distribution is due to $\mathrm{H}$ atom scattering before and after crossing the adsorption barrier. Upper and lower right panels of Figure 6.14 show the agreement of theoretical calculation with experiment at higher normal incidence energies. Nearly all the $\mathrm{H}$ atoms have enough normal incidence energy to cross the barrier, and only the slow component is observed in the translational energy loss and scattering angle distribution. Figure 6.15 shows energy loss obtained from AIMD simulations as a function of the minimum C-H distance. Clear differences are seen for $\mathrm{H}$ atoms scatterings before and after crossing the adsorption barrier. Atoms that cross the barrier have a significantly shorter minimum $\mathrm{C}-\mathrm{H}$ distance, indicating formation of a transient $\mathrm{C}-\mathrm{H}$ bond.

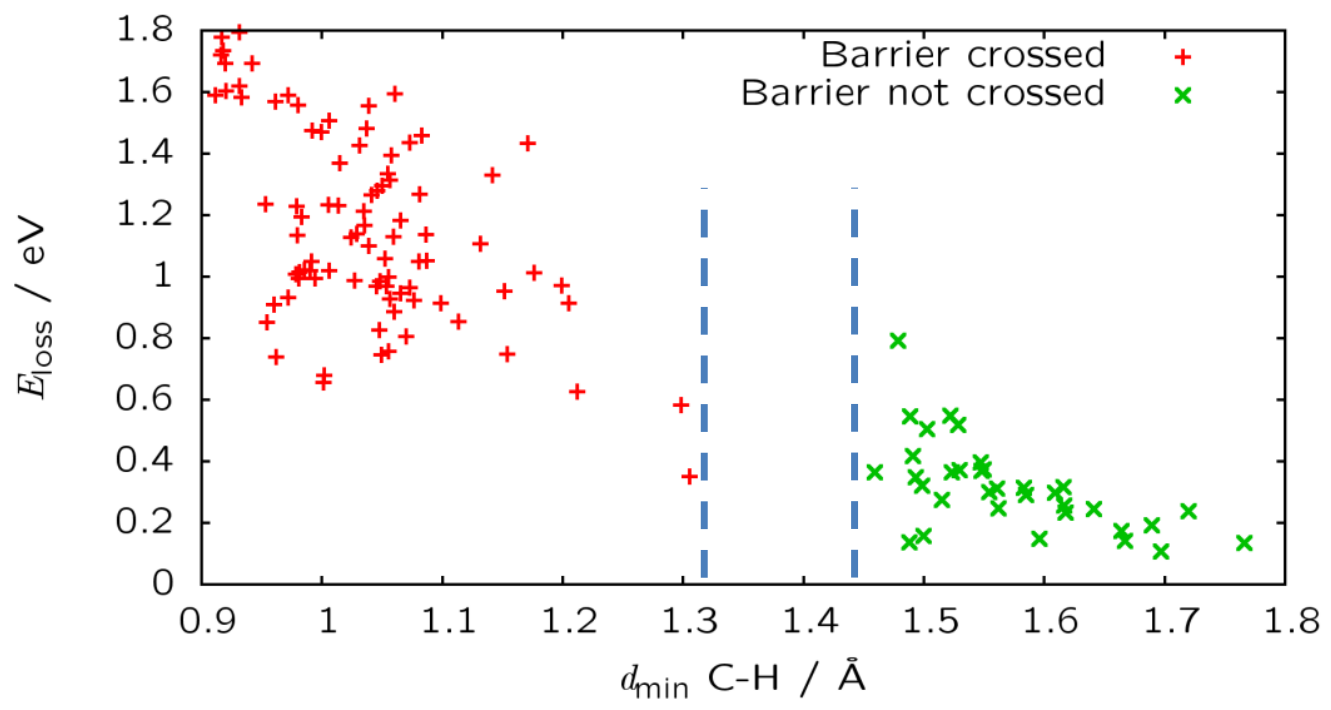

Figure 6.15: Minimum C-H distance obtained from AIMD simulation as a function of energy losses. The incidence energy is $1.92 \mathrm{eV}$, and the incidence angle is $52^{\circ}$. Blue dashed lines indicate the separation of the fast and slow components (138). 


\subsubsection{The fast component: Atoms scattering on a corrugated surface}

In this section, I will discuss the scattering dynamics of the fast component. As shown in Figures 6.4 to 6.10 , the fast and slow components simultaneously exist for most of the incidence conditions that were employed in the study. In order to characterize both components, they must be separated in the 2D translational energy and scattering angle distribution. In Figure 6.16, a separating straight line is drawn along the valley between the two components. I carried out separate quantitative analysis for the two components. For different incidence conditions, the dividing line is adjusted to keep it along the valley.

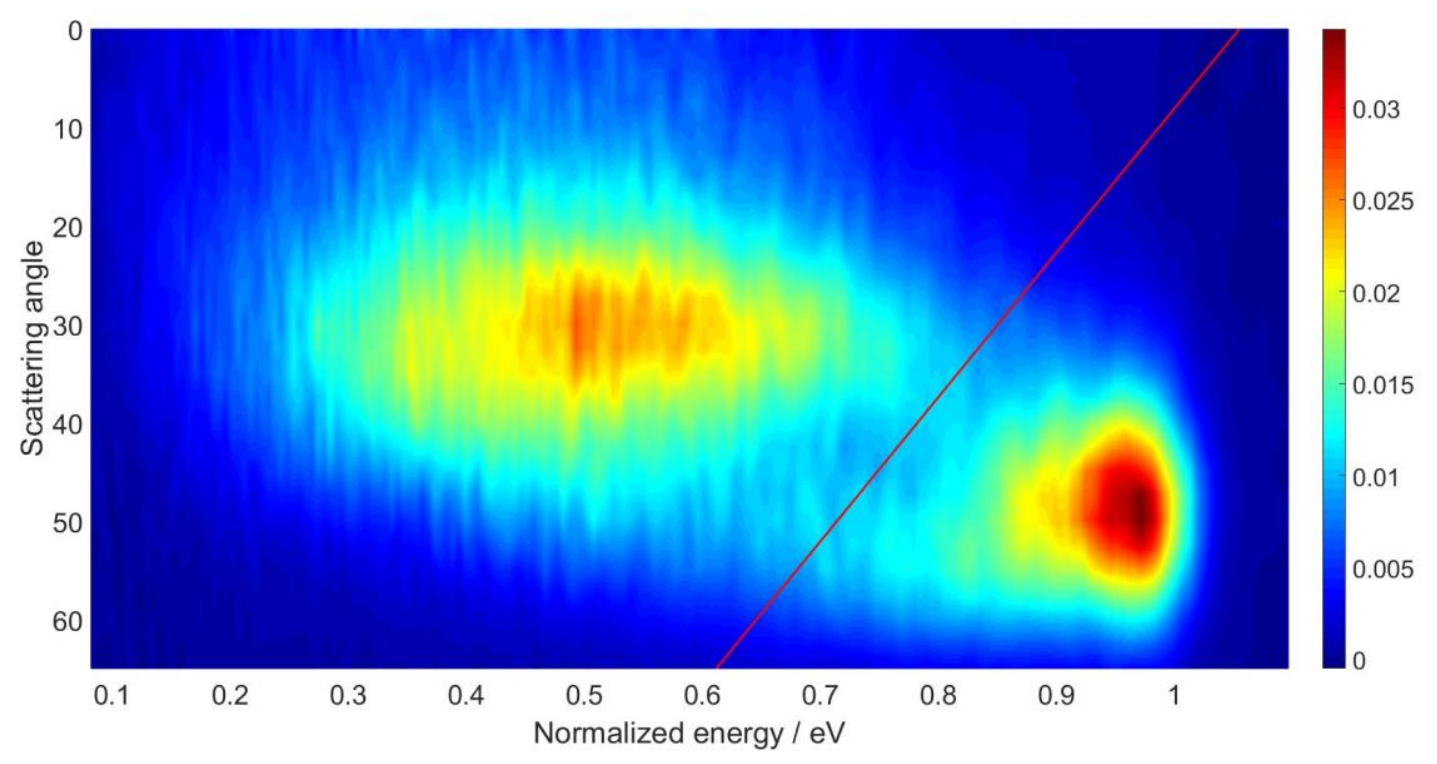

Figure 6.16: Separation of the fast and slow components in the $2 D$ translational energy and scattering angle distribution. The incidence energy and incidence angle are $1.92 \mathrm{eV}$ and $48^{\circ}$. 


\section{Experimentally measured translational energy loss and scattering angle:}
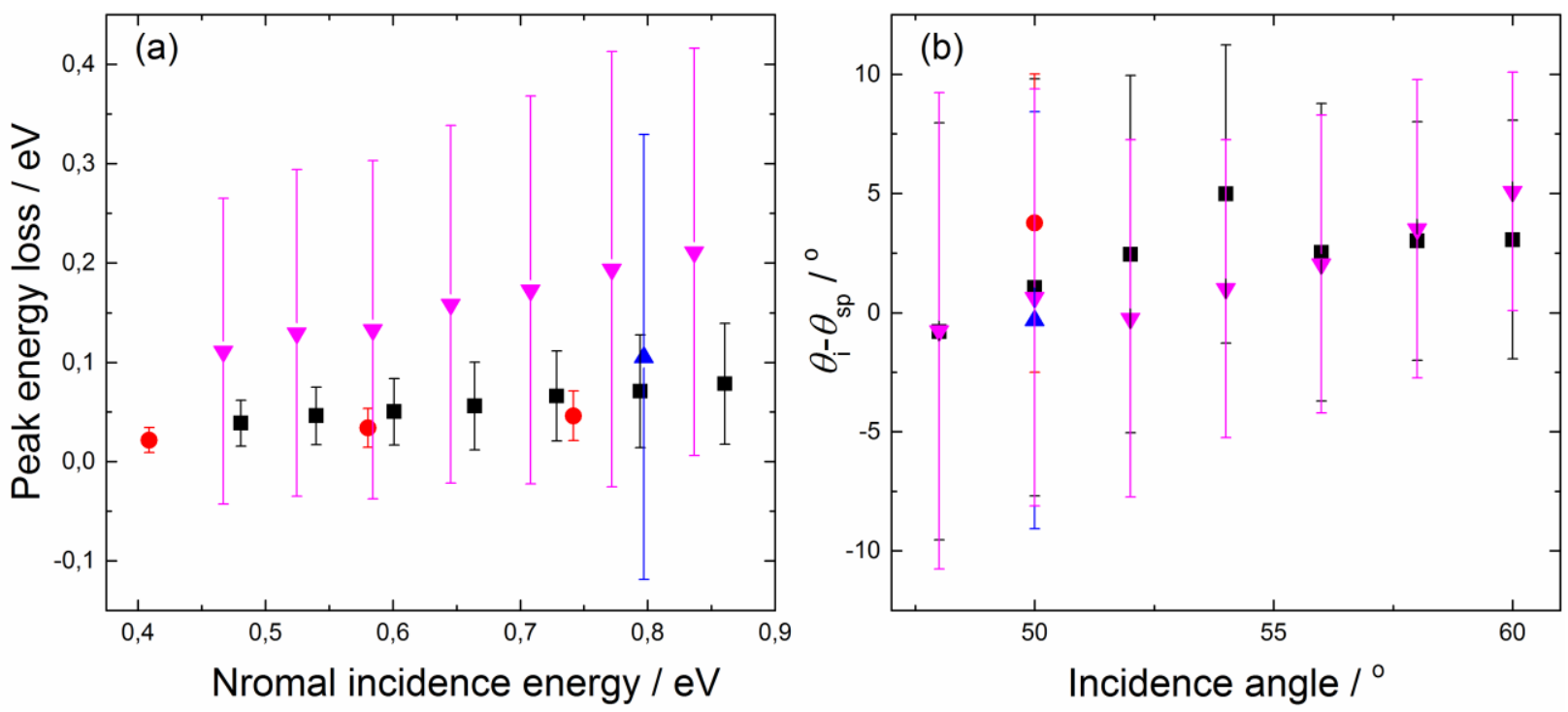

Figure 6.18: Panel (a): Peak translational energy loss and FWHM of the translational energy distribution for the fast component of $H / D$ atoms scattering from epitaxial graphene. Panel $(b)$ : Peak angular difference $\left(\theta_{\mathrm{i}}-\theta_{\mathrm{sp}}\right.$, difference between the incidence angle and scattering angle at the peak) and FWHM of the scattering angle distribution of H/D atoms scattering from epitaxial graphene. The red circles represent $0.99 \mathrm{eV} \mathrm{H}$ atom scattering. The black squares represent 1.92 $\mathrm{eV} \mathrm{H}$ atom scattering. The blue triangle represents $1.92 \mathrm{eV} \mathrm{H}$ atom scattering at $600 \mathrm{~K}$ surface temperature. The magenta triangles represent $1.87 \mathrm{eV} D$ atom scattering.

Figure 6.18 (a) shows the peak energy loss and FWHM of the translational energy distributions for the fast component of $\mathrm{H}$ and $\mathrm{D}$ atoms scattering from graphene under various incidence conditions. The red circles represent $0.99 \mathrm{eV} \mathrm{H}$ atom scattering. The black squares represent 1.92 $\mathrm{eV} \mathrm{H}$ atom scattering. The blue triangle represents $1.92 \mathrm{eV} \mathrm{H}$ atom scattering at $600 \mathrm{~K}$ surface temperature. The magenta triangles represent $1.87 \mathrm{eV} \mathrm{D}$ atom scattering. As shown in the figure, the peak energy loss for the fast component is small and increases with the total incidence energy, the normal incidence energy and the surface temperature. Substituting H with D also increases the peak energy loss. The FWHM of the translational energy distributions for the fast component is narrow and also increase with the total incidence energy, the normal incidence energy and the surface temperature. D atoms exhibit larger scattering angle FWHM than that of $\mathrm{H}$ atoms. 
Figure 6.18 (b) shows the difference between incidence angle and peak scattering angle of the fast component $\left(\theta_{\mathrm{i}}-\theta_{\mathrm{sp}}\right)$ and FWHM of the scattering angle distribution. Zero difference indicates specular scattering. The incidence conditions are the same with those in Panel (a). For all incidence conditions, the angular differences are small (maximum $5^{\circ}$ ) and the FWHMs are narrow, showing the scattering is near-specular. At high incidence angles, the angle differences are above zero (super-specular). As the incidence angle is decreased, the angle difference also decreases. Surface temperature and isotope substitution does not affect the peak scattering angle.

Discussion on the fast component: First, the fast component exhibits minor energy loss and is quasi-specular for all incidence conditions. The spread of energy loss and scattering angle are narrow, indicating a single bounce elastic scattering process. Figure 6.19 shows the number of $\mathrm{H}$ atom bounces on epitaxial graphene as a function of the translational energy loss and scattering angle, obtained from AIMD calculation. The incidence energy and incidence angle are $1.92 \mathrm{eV}$ and $52^{\circ}$, respectively. It can be seen from Figure 6.19 that nearly all the fast component trajectories only have one bounce. The AIMD calculation provides theoretical support that the scattering is a single bounce elastic scattering process.
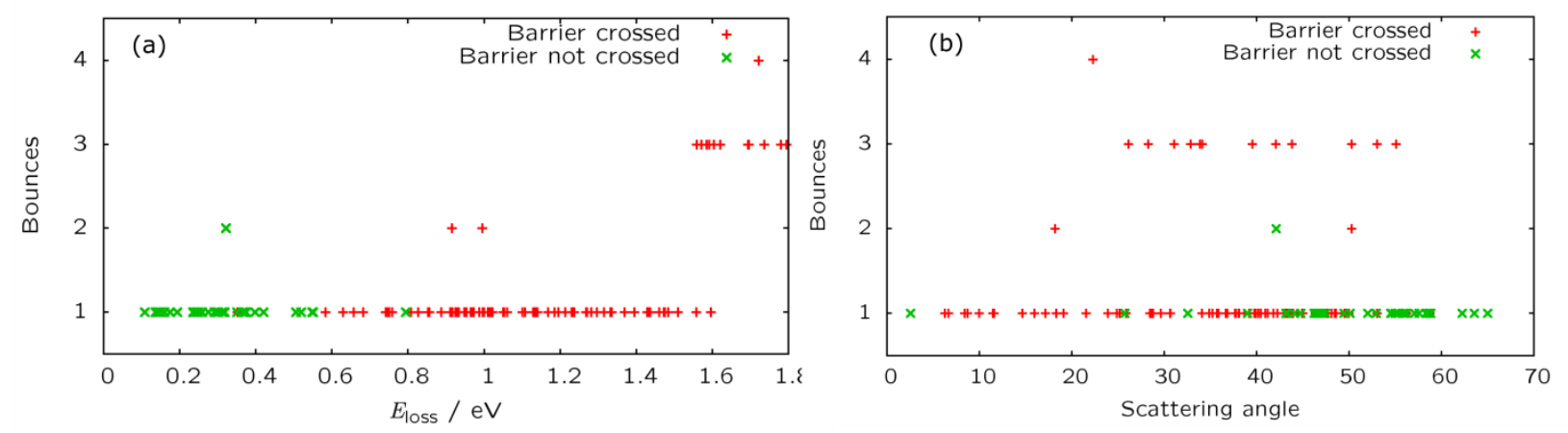

Figure 6.19: Number of bounces of the $H$ atom scattering on epitaxial graphene from AIMD calculation. The incidence energy and incidence angle are $1.92 \mathrm{eV}$ and $52^{\circ}$ (138).

Second, the energy loss increases with normal incidence energy at constant total incidence energy, and also with total incidence energy at constant incidence angle. This indicates that fast component is similar to noble gas atoms scattering on metal surfaces, which was introduced in Chapter 2. Energy transfer is along the surface normal direction, whereas parallel energy is conserved. For a flat surface, the scattering angle should be larger than the incidence angle. 
However, we observed scattering angles smaller than the incidence angles (Figure 6.18), indicating that the hydrogen atom is not scattered on a flat surface, but a corrugated one.

Figure 6.20 illustrates atoms scattering on a corrugated surface. Here, we only consider the forward scattering, since only forward in plane scattering signals are measured in the experiment. The right side of Figure 6.20 shows scattering on the top site of the surface. $\theta_{\mathrm{i}}$ and $\theta_{\mathrm{s}}$ are incidence angle and scattering angle, respectively. It is the same as atom scattering on a flat surface, and fulfills the relation $\theta_{\mathrm{s}}>\theta_{\mathrm{i}}$. The left side of Figure 6.20 shows the atom scattering on the uphill site (black curves) of the surface. The effective incidence angle is $\theta_{\mathrm{i}}^{*}=\theta_{\mathrm{i}}-\theta$. Even though the effective scattering angle $\theta_{\mathrm{s}}^{*}$ is larger than the effective incidence angle is $\theta_{\mathrm{i}}^{*}$, the measured scattering angle $\theta_{\mathrm{sm}}=\theta_{\mathrm{s}}{ } \theta$ could still be smaller than the incidence angle $\theta_{\mathrm{i}}$. Scattering on downhill sites (red curves) is opposite and its measured scattering angle $\theta_{\mathrm{sm}}$ is always bigger than incidence angle $\theta_{\mathrm{i}}$. For larger incidence angles, the atom will mostly be scattered on the uphill sites. While for smaller incidence angles, the atom has chance to be scattered on a downhill site. This explains the decrease of the angular difference between incidence and peak scattering angles, when the incidence angle is changed from $60^{\circ} \sim 48^{\circ}$ (Figure 6.18). Figure 6.21 shows the PECs for $\mathrm{H}$ atom interaction with the coronene molecule at three different sites from ROCCSD(T) calculation (39). Clear differences in potential energies can be seen for the three different sites, providing theoretical support for a corrugated surface for $\mathrm{H}$ atom scattering. Based on the discussion above, we conclude that the fast component originates from single bounce elastic scattering on a corrugated surface. We can only present qualitative descriptions of the fast component. For a quantitative description, more theoretical work is needed.

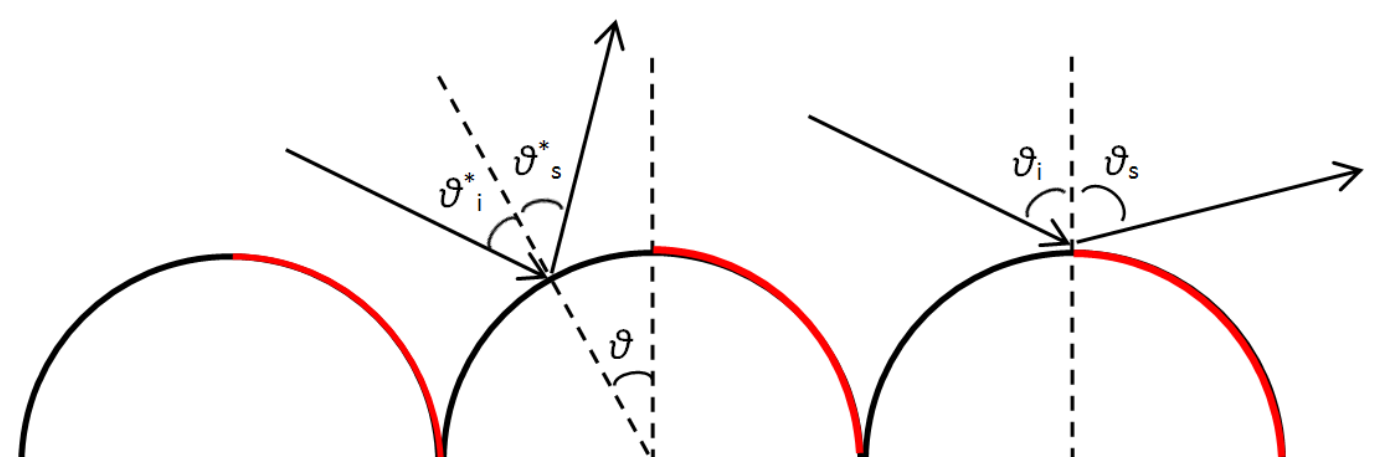

Figure 6.20: Illustration of H atoms scattering on a corrugated surface. 
(a)

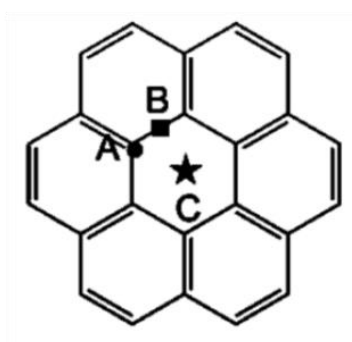

(b)

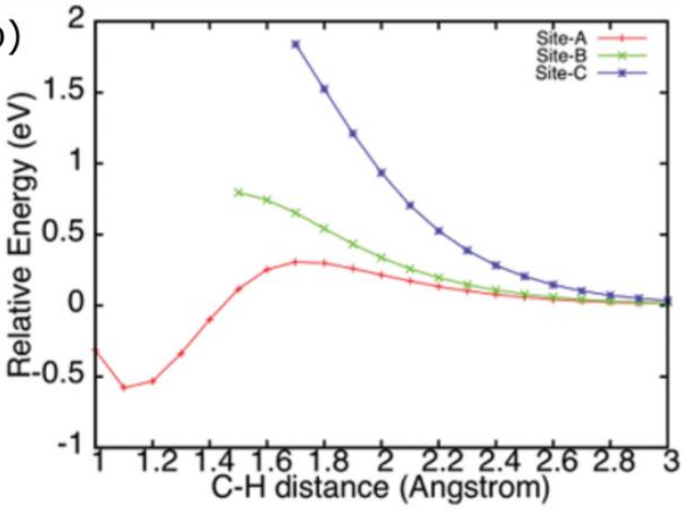

Figure 6.21: PECs of a $\mathrm{H}$ atom adsorption on a coronene molecule, obtained from ROCCSD(T) calculation. Panel (a): indication of adsorption sites used in the calculation. Panel (b): PECs as a function of $\mathrm{C}-\mathrm{H}$ distance. Reprinted figure with permission from Ref. (39). Copyright (2012) American Chemical Society.

\subsubsection{The slow component: Transient reaction complex formation, tug-of-war inelastic scattering, surface IVR and dynamical steering effect}

Experimentally measured translational energy loss and scattering angle: In this section, the scattering dynamics of the slow component will be discussed. Figure 6.21 shows the average energy loss of the slow component. Panel (a) displays the energy loss of $\mathrm{H}$ and D atoms at different incidence angles but the same incidence energy. Incidence energies are $1.92 \mathrm{eV}$ and $1.87 \mathrm{eV}$ for $\mathrm{H}$ and $\mathrm{D}$, respectively. Incidence angles are varied between $60^{\circ} \sim 30^{\circ}$, corresponding to normal incidence energies of $0.48 \sim 1.44 \mathrm{eV}$ for $\mathrm{H}$ and $0.47 \sim 1.40 \mathrm{eV}$ for D. Error bars indicate the FWHM in energy loss. Unlike the fast component, the slow component exhibits a large energy loss and does not have any significant dependence on the incidence angle. Averaged over all incidence angles, $\mathrm{H}$ atoms lose $0.93 \mathrm{eV}$ (48\% of their incidence energy) and D atoms lose $0.97 \mathrm{eV}$ ( $52 \%$ of their incidence energy). The FWHM of the energy loss does not exhibit a strong dependence on the incidence angle either. Averaged over all the incidence angles, the FWHMs are $0.93 \mathrm{eV}$ and $0.81 \mathrm{eV}$ for $\mathrm{H}$ and $\mathrm{D}$ atoms, respectively. Figure 6.21 (b) shows the average energy loss of $\mathrm{H}$ and $\mathrm{D}$ atoms at different incidence energies and surface temperatures but the same incidence angle. The incidence angle is $50^{\circ}\left(49.1^{\circ}\right.$ for $3.25 \mathrm{eV} \mathrm{D}$ atoms). Increasing surface temperature changes neither the average energy loss nor the FWHM of the energy loss, indicating that a higher surface temperature only promotes the crossing of the adsorption barrier, 
but has no effect on the energy loss that occurs after the barrier has been overcome. Increasing the incidence energy raises the average energy loss, but the percentage energy loss $\left(\left\langle E_{\text {loss }}\right\rangle / E_{\mathrm{i}}\right)$ decreases, as shown in Table 6.3.

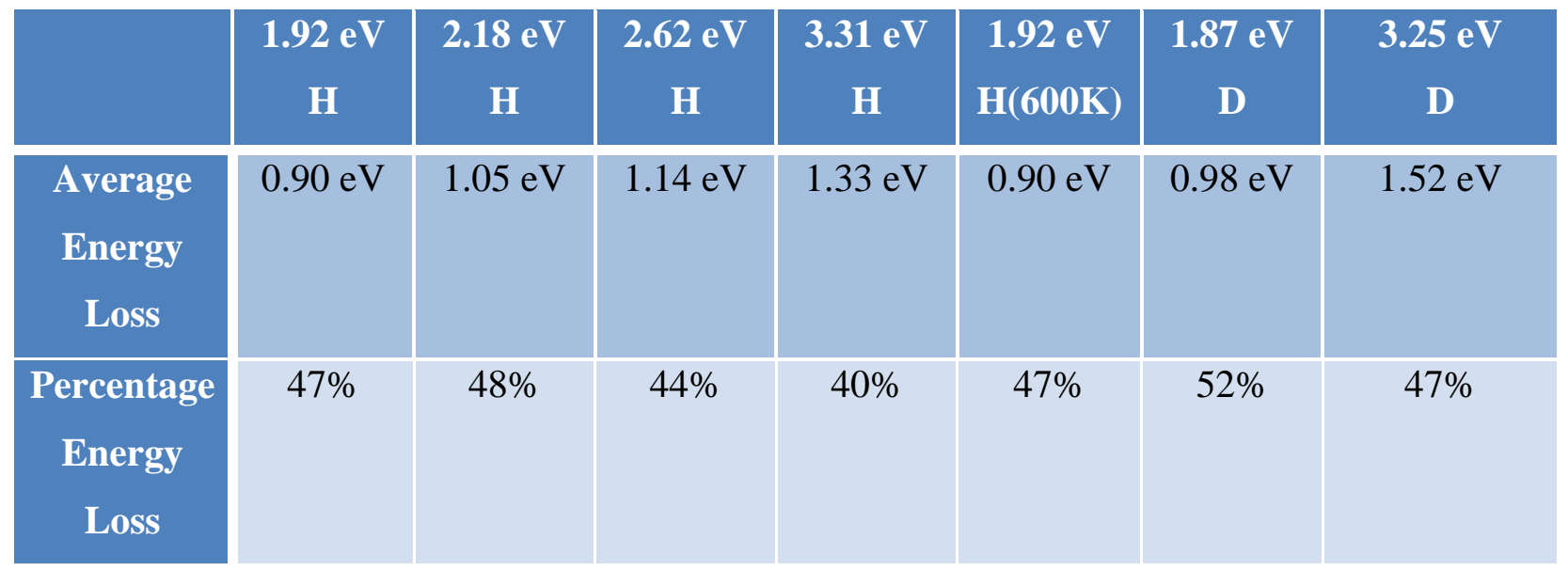

Table 6.3: Energy loss of the slow component at different incidence energies and surface temperatures.
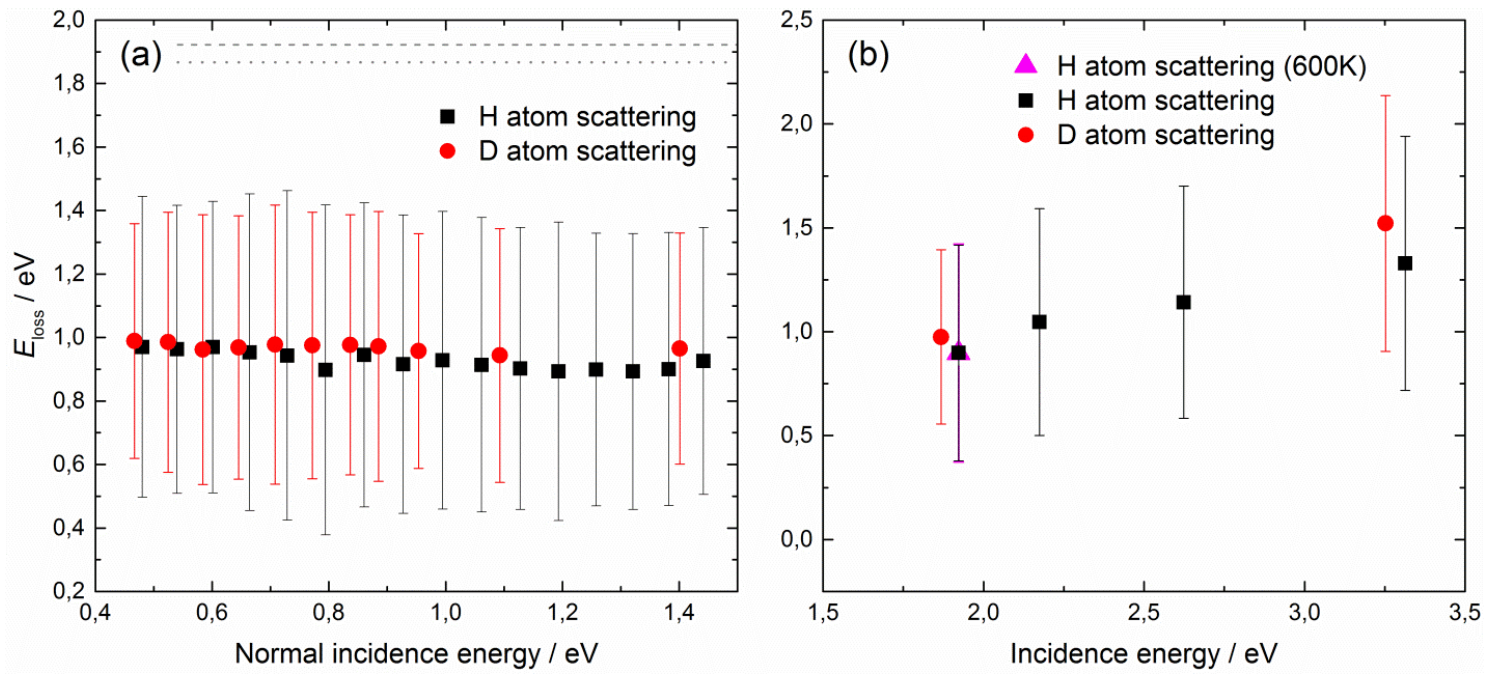

Figure 6.21: Average energy loss of the slow component. Panel (a): Average energy loss of $H$ and $D$ atoms at different normal incidence energies but the same total incidence energy. Incidence energies are $1.92 \mathrm{eV}$ (grey dashed line) and $1.87 \mathrm{eV}$ (grey dot line) for $\mathrm{H}$ and D atoms respectively. Error bars indicate FWHM of the energy loss. Panel (b): Average energy loss of $H$ and $D$ atoms at different incidence energies and surface temperatures but the same incidence angle. The incidence angles are $50^{\circ}\left(49.1^{\circ}\right.$ for $3.25 \mathrm{eV} \mathrm{D}$ atoms). The surface temperatures are $300 \mathrm{~K}$ and $600 \mathrm{~K}$. 

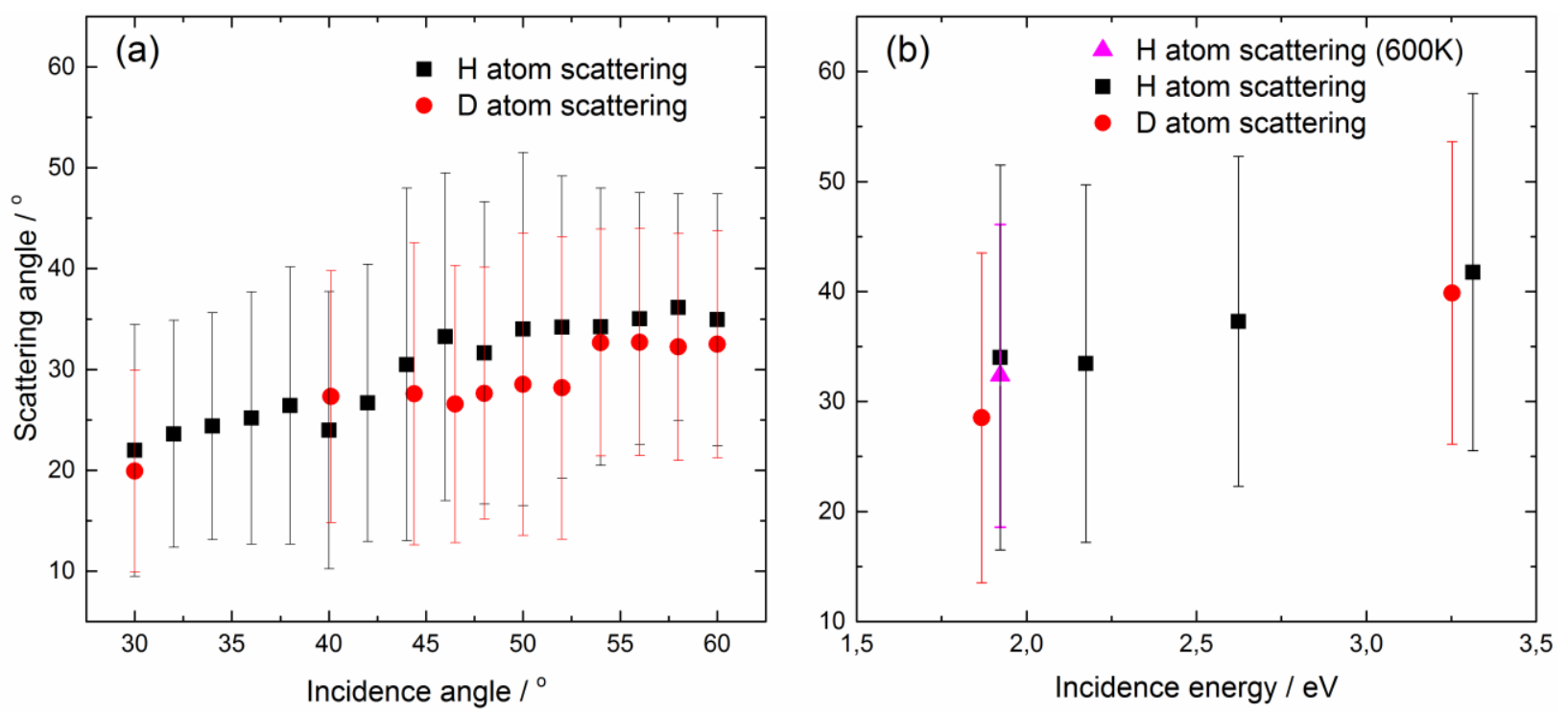

Figure 6.22: Average scattering angle of the slow component. Panel (a): Average scattering angle of $H$ and $D$ atoms at different incidence angles but the same incidence energy. Incidence energies are $1.92 \mathrm{eV}$ and $1.87 \mathrm{eV}$ for $\mathrm{H}$ and $\mathrm{D}$ atoms, respectively. The error bars indicate the FWHM of the scattering angle. Panel (b): Average scattering angle of $H$ and $D$ atoms at different incidence energies and surface temperatures but the same incidence angle. The incidence angles are $50^{\circ}\left(49.1^{\circ}\right.$ for $3.25 \mathrm{eV} \mathrm{D}$ atoms). The surface temperatures are $300 \mathrm{~K}$ and $600 K$.

Figure 6.22 shows the average scattering angle of the slow component. Panel (a) displays the average scattering angles of $\mathrm{H}$ and $\mathrm{D}$ atoms at different incidence angles but the same incidence energy. The incidence energies are $1.92 \mathrm{eV}$ and $1.87 \mathrm{eV}$ for $\mathrm{H}$ and $\mathrm{D}$, respectively. Error bars indicate FWHM of the scattering angle. The average scattering angle decreases from $35^{\circ}$ to $22^{\circ}$ for $\mathrm{H}$ atoms as the incidence angle is changed from $60^{\circ}$ to $48^{\circ}$. For $\mathrm{D}$ atoms, the average scattering angle decreases from $32^{\circ}$ to $20^{\circ}$. The FWHM of the scattering angle of the slow component is larger than that of the fast component, but the scattering still exhibits preferential directions. Averaged over all the incidence angles in Figure 6.22 (a), the FWHMs are $27.5^{\circ}$ and $25^{\circ}$ for $\mathrm{H}$ and $\mathrm{D}$ atoms scattering, respectively. Figure 6.22 (b) displays the average scattering angle of $\mathrm{H}$ and $\mathrm{D}$ atoms at different incidence energies and surface temperatures but the same incidence angle. The incidence angles are $50^{\circ}\left(49.1^{\circ}\right.$ for $3.25 \mathrm{eV} \mathrm{D}$ atoms). The average scattering angle and FWHM do not depend on the surface temperature as discussed above. The average scattering angle increases about $8^{\circ}$ when the incidence energy is changed from $1.92 \mathrm{eV}$ 
to $3.31 \mathrm{eV}$ for $\mathrm{H}$ atom scattering. For $\mathrm{D}$ atom scattering, the average scattering angle increases about $11^{\circ}$ when the incidence energy is increased from $1.87 \mathrm{eV}$ to $3.25 \mathrm{eV}$. However, the FWHM is nearly the same for different incidence energies.

Large and rapid energy transfer induced by transient reaction complex formation, tug-ofwar inelastic scattering and surface IVR (intramolecular vibrational energy redistribution): By comparing the above presented results to AIMD simulations, we can reach a pictorial understanding of the scattering dynamics for the slow component.
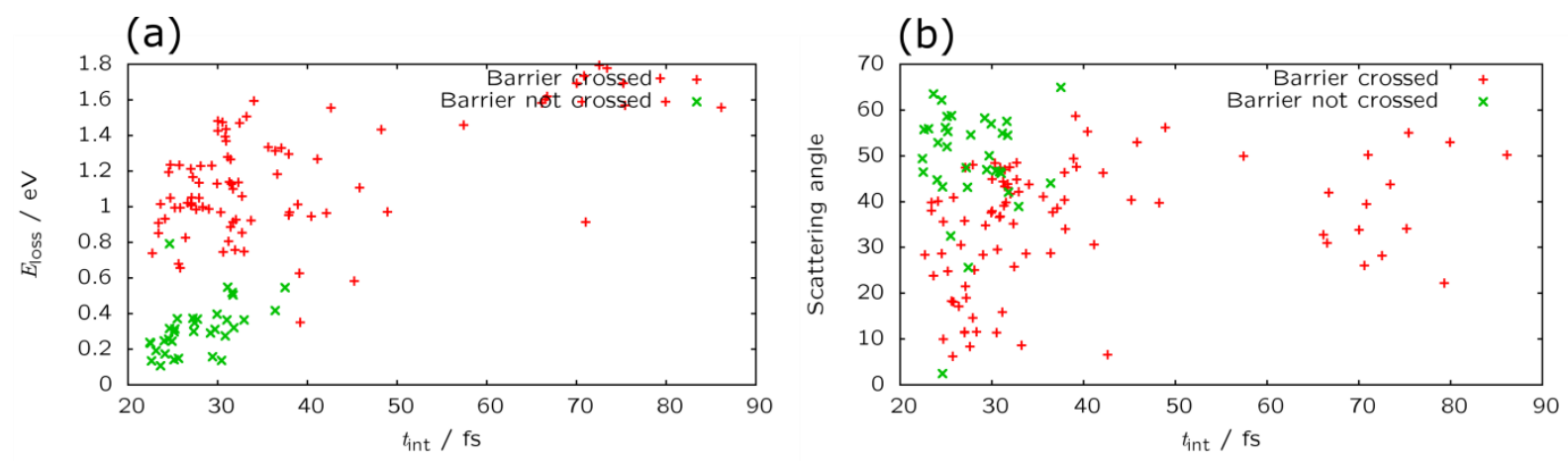

(c)

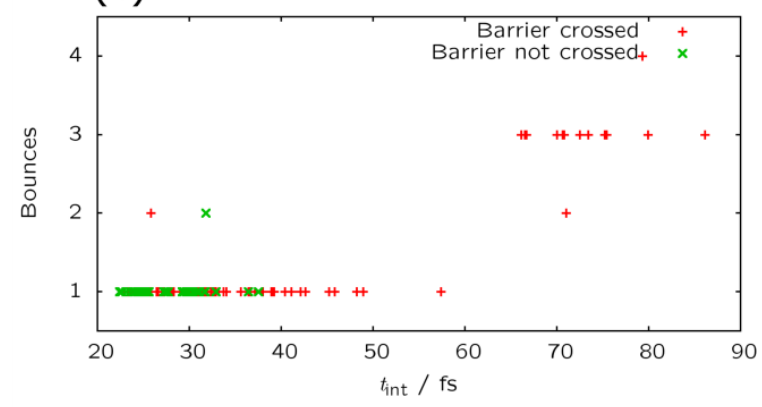

Figure 6.23: $H$ atom energy loss, scattering angle and number of bounces as a function of interaction time from AIMD calculation. The incidence energy and angle are $1.92 \mathrm{eV}$ and $52^{\circ}$. The incidence direction is parallel to the plane defined by the $C$ - $C$ bond and surface normal (138).

The AIMD calculations predict that most of the barrier crossed trajectories (82\%) only have one bounce on the graphene surface, as shown in Figure 6.19. Single bounce scattering suggests that the interaction time is very short at the incidence energies used in the above presented experiments. Considering the adsorption barrier is located about $1.7 \AA$ above the $\mathrm{C}$ atom, the interaction time of single bounce scattering (crossing the barrier twice) is on the order of several 
tens of femtoseconds for all the experimental incidence conditions. Figure 6.23 shows the theoretically calculated energy losses, scattering angles and number of $\mathrm{H}$ atom bounces as a function of interaction time. The incidence energy and angle are $1.92 \mathrm{eV}$ and $52^{\circ}$. The incidence direction is parallel to the plane defined by the C-C bond and surface normal. The interaction time is defined as the time duration during which the H-graphene distance is smaller than $2.2 \AA$.

As shown in Figure 6.23, the interaction times for single bounce barrier crossed trajectories are between $20 \sim 50 \mathrm{fs}$, and the average value is $35 \mathrm{fs}$. The energy loss and scattering angle have no dependence on the interaction time. The theoretically predicted interaction time for the fast component is similar to that of the slow component, but the energy loss for the slow component is much larger than that for the fast component. For $1.92 \mathrm{eV} \mathrm{H}$ atom scattering, the atoms lose on average $0.9 \mathrm{eV}$ in about $35 \mathrm{fs}$, corresponding to a surprisingly high average power of $10^{19} \mathrm{~W}$ per mole incidence atom. Further analysis of the AIMD trajectories gives insight of the large and rapid energy transfer.

Figure 6.24 shows the results of an $\mathrm{H}$ atom in-plane scattering trajectory, obtained from AIMD calculation. The $\mathrm{H}$ atom only collides with one $\mathrm{C}$ atom during the scattering. The incidence energy and angle are $1.92 \mathrm{eV}$ and $52^{\circ}$. The incidence direction is in the plane defined by the surface normal and the $\mathrm{C}-\mathrm{C}$ bond. Panel (a) shows the distance between the $\mathrm{H}$ atom and the graphene surface $\left(\mathrm{Z}_{\mathrm{H}}\right)$, and the minimum $\mathrm{C}-\mathrm{H}$ distance $\left(\mathrm{M}_{\mathrm{C}-\mathrm{H}}\right)$ as a function of the simulation time. Panels (b) and (c) show different kinds of energy loss (or energy gain) of the system as a function of the simulation time. In Panel (b), the black curve represents the energy loss of the $\mathrm{H}$ atom $\left(\mathrm{E}_{\mathrm{loss}}\right)$, the red curve represents the normal energy loss of the $\mathrm{H}$ atom $\left(\mathrm{E}_{\mathrm{lossHz}}\right)$, the blue curve represents the potential energy gain in the graphene slab $\left(U_{\text {slab}}\right)$, and the magenta curve represents the kinetic energy gain of $\mathrm{C}$ atoms in the graphene slab ( $\left.\mathrm{E}_{\text {slab }}\right)$. In panel (c), the magenta curve represents the kinetic energy gain of $\mathrm{C}$ atoms in the graphene slab $\left(\mathrm{E}_{\text {slab}}\right.$, same as that in Panel (b)), the red curve represent the kinetic energy gain of the colliding $C$ atom $\left(E_{C}\right)$, the blue curve represents the kinetic energy gain of the nearest $\mathrm{C}$ atoms ( 3 atoms) to the colliding $\mathrm{C}$ atom $\left(E_{\mathrm{nn}}\right)$, and the black curve represents the kinetic energy gain of the next nearest $\mathrm{C}$ atoms (6 atoms) to the colliding $\mathrm{C}$ atom $\left(\mathrm{E}_{\mathrm{nnn}}\right)$. 


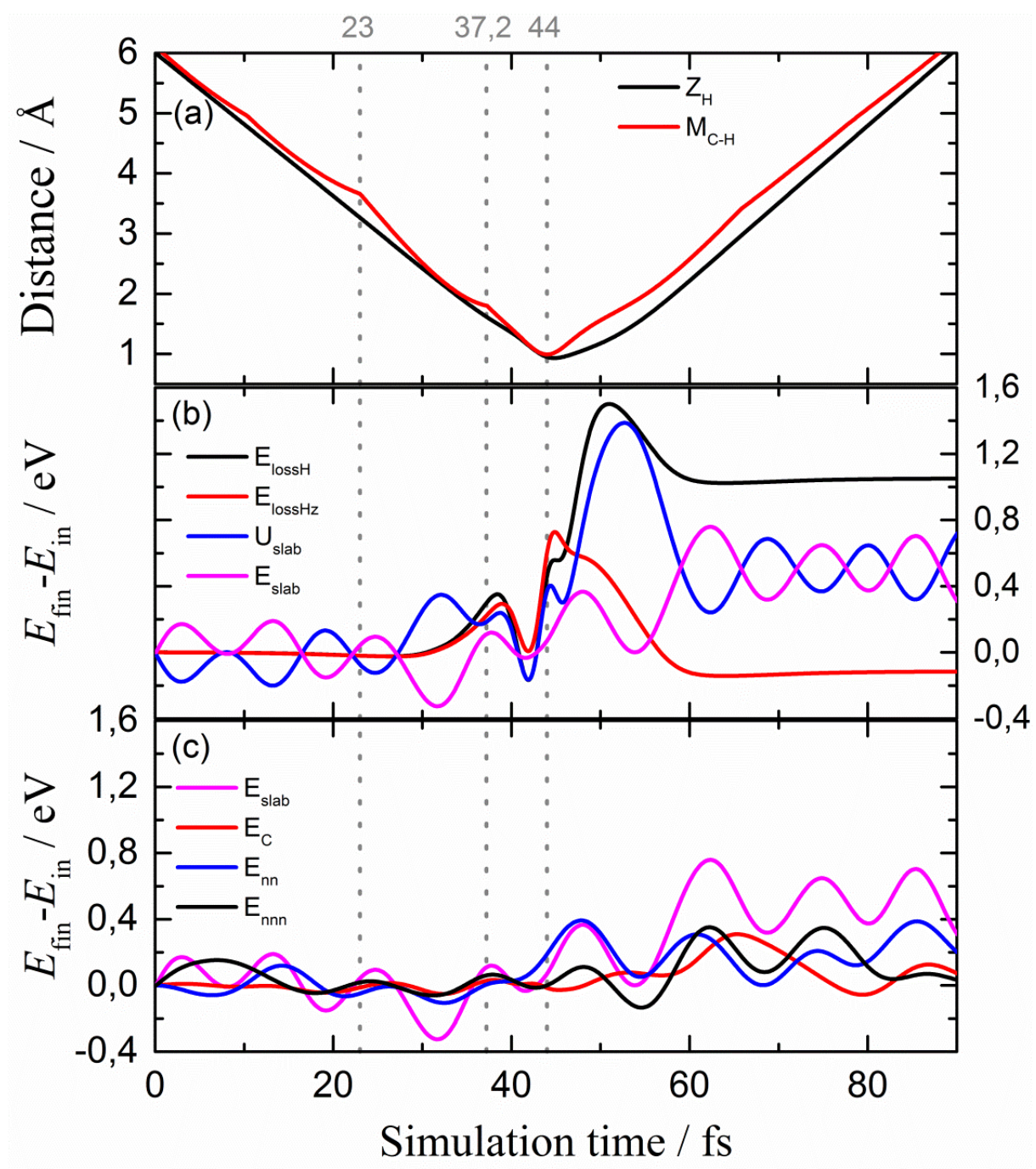

Figure 6.24: Minimum distance and energy loss (gain) of a single bounce in-plane scattering trajectory for an $\mathrm{H}$ atom on graphene, obtained from AIMD simulation (138). See the text for detailed description.

As shown in Figure 6.24 (a), $\mathrm{Z}_{\mathrm{H}}$ decreases smoothly in the beginning of the trajectory. At simulation time $t=44.8 \mathrm{fs}, \mathrm{Z}_{\mathrm{H}}$ reaches its minimum $(0.93 \AA)$ and then starts to increase, indicating a single bounce collision with the surface. However, $\mathrm{M}_{\mathrm{C}-\mathrm{H}}$ does not change smoothly. This arises from the fact that the $\mathrm{H}$ atom flies over different $\mathrm{C}$ atoms during the collision, shifting its closest $\mathrm{C}$ atom. Gray dotted lines shown at $t=23$ and $37.2 \mathrm{fs}$ represent two shifts in the closest $\mathrm{C}$ atom. The gray dotted line shown at $t=44 \mathrm{fs}$ indicates the minimum $\mathrm{C}-\mathrm{H}$ distance. 
As shown in Figure 6.24 (b), the $\mathrm{H}$ atom starts to lose energy at $t=30 \mathrm{fs}$, predominantly along the surface normal direction indicating the atom is climbing up the adsorption barrier. The oscillation of $\mathrm{U}_{\text {slab }}$ and $\mathrm{E}_{\text {slab }}$ before $t=30 \mathrm{fs}$ is due to the thermal motion of the graphene. At $t=38.9 \mathrm{fs}$, the normal energy loss reaches a local maximum and then starts to decrease again, indicating that the $\mathrm{H}$ atom has crossed the adsorption barrier and gets accelerated by the chemisorption well. Note that the closest $\mathrm{C}$ atom shifts once while the $\mathrm{H}$ atom is climbing up the adsorption barrier, indicating the $\mathrm{H}$ atom crosses a dynamical barrier in this trajectory. At $t=41.9$ fs, both the total and normal energy loss of $\mathrm{H}$ reach their minimum, indicating the $\mathrm{H}$ atom arrives at the bottom of the chemisorption well. At $t=44 \mathrm{fs}$, the $\mathrm{C}-\mathrm{H}$ distance reaches its minimum (shown in Panel(a)), but the $\mathrm{H}$ atom continues to move towards the graphene until $t=44.8 \mathrm{fs}$, at which time it loses all its normal incidence energy $(0.73 \mathrm{eV})$ and bounces back from the graphene. It is interesting that the $\mathrm{H}$ atom continues to lose its energy after it bounces back, which appears contradictory with common sense. The atom should start to gain some energy after being bounced back, since it will be accelerated by the chemisorption well. However, $\mathrm{H}$ atom continues to lose energy until $t=51 \mathrm{fs}$, at which time it crosses the adsorption barrier again and flies away.

A close look at the potential and kinetic energy gain in the graphene slab provides insights into the apparent contradiction. After the $\mathrm{H}$ atom is bounced back, the kinetic energy in the slab does not change very much, while the potential energy in the graphene slab increases quite a lot, indicating a large portion of energy transferred from the $\mathrm{H}$ atom is used to deform the graphene slab. When the $\mathrm{H}$ atom bounces back from the surface, the attractive force between the $\mathrm{H}$ and the bonding $\mathrm{C}$ atom drags the $\mathrm{C}$ atom away from the surface, leading to deformation of the graphene. This dynamics is similar to that of the tug-of-war mechanism observed in the gas phase collision of $\mathrm{H}$ and $\mathrm{D}_{2}(6,7,147)$. In commonly accepted mechanisms, the vibrational excitation of molecules in gas phase collisions occurs via compressing the bond. For a tug-of-war inelastic collision of $\mathrm{H}$ and $\mathrm{D}_{2}$, the incoming $\mathrm{H}$ atom first forms a transient $\mathrm{H}-\mathrm{D}$ bond with the molecule, and then loses a large amount of its initial kinetic energy by stretching the D-D bond during its way out. The energy loss is mainly due to the attractive part of the potential, not the repulsive part. The interaction time of tug-of-war inelastic collision is also on the same order with $\mathrm{H}$ atom scattering from graphene. Both experiments have a short interaction time of a few tens of femtoseconds, which further supports the similarity in the dynamics of the two processes. The 
tug-of-war mechanism could explain the small isotope effect shown in Figure 6.21 and 6.22. Since a large portion of the energy loss arises from the graphene deformation, the mass of the incidence atom does not have a large effect on the final energy loss.

Figure 6.24 (c) shows the energy gain for different $\mathrm{C}$ atoms in the graphene slab. It is obvious that the energy is not only transferred to the colliding $\mathrm{C}$ atom, but also simultaneously to its neighboring $\mathrm{C}$ atoms. In fact, the next nearest $\mathrm{C}$ atoms ( 3 atoms) even gain kinetic energy earlier than the colliding $\mathrm{C}$ atom. This simultaneous energy transfer to several $\mathrm{C}$ atoms gives rise to the large and rapid energy loss, since energy can be dissipated to many degrees of freedom at the same time, and will not flow back to the $\mathrm{H}$ atom easily. These dynamics arise from the special electronic structure of graphene.

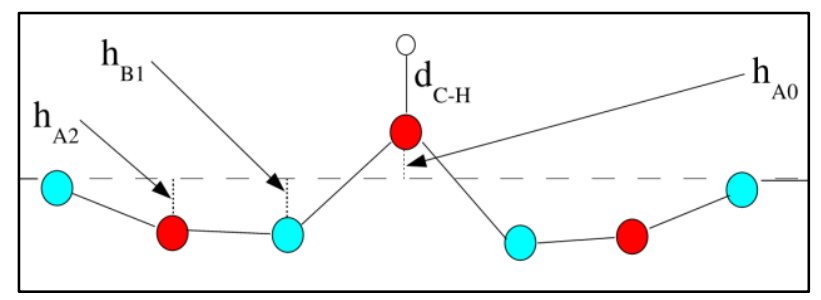

Figure 6.25: Equilibrium configuration of a $H$ atom adsorption on graphene obtained from DFT calculation. The empty circle represents the $H$ atom. The red and cyan circles represent $C$ atoms on the two respective sub-lattices. The dashed line indicates the original graphene plane. Reprinted figure with permission from Ref. (110). Copyright (2012) American Chemical Society.

Figure 6.25 shows the equilibrium configuration of $\mathrm{H}$ atom adsorption on graphene (110). It can be clearly seen that not only the bonding $\mathrm{C}$ atom in the $\mathrm{C}-\mathrm{H}$ bond puckers up, but also the neighboring $\mathrm{C}$ atoms are substantially displaced from their original positions. The displacements shown in Figure 6.25 are calculated as $\mathrm{h}_{\mathrm{A} 0}=0.257 \AA, \mathrm{h}_{\mathrm{B} 1}=-0.047 \AA$ and $\mathrm{h}_{\mathrm{A} 2}=-0.036 \AA$. Although different calculations may predict different values, the simultaneous displacements of several $\mathrm{C}$ atoms are common. The simultaneous displacement of the neighboring $\mathrm{C}$ atoms is attributed to the delocalized $\pi$ resonance structure of graphene (112). Three outer shell electrons of the $\mathrm{C}$ atom are involved in forming in-plane $\mathrm{C}-\mathrm{C} \sigma$ bonds via $\mathrm{sp}^{2}$ hybridization, but the fourth $\mathrm{p}$ orbital electron covalently binds with other neighboring $\mathrm{C}$ atoms via formation of a $\pi$ bond. Adsorption of a $\mathrm{H}$ atom on one sub-lattice (A) removes a $\mathrm{p}$ orbital from the $\pi$ system, and sets free an unpaired electron on the other sub-lattice (B). This electron will delocalize on the sub-lattice (B), 
and causes $\mathrm{sp}^{2}$ to $\mathrm{sp}^{3}$ rehybridization of the $\mathrm{C}$ atoms, leading to the simultaneous displacement. The unpaired electron induces magnetic moments on graphene, which can be used to characterize the scale of the delocalization. Figure 6.26 shows theoretically calculated (112) and experimentally measured (148) magnetic moments induced by single $\mathrm{H}$ atom adsorption on graphene. The unpaired electron can delocalize onto $\mathrm{C}$ atoms nanometers away from the adsorbed $\mathrm{H}$ atom, indicating the adsorption induced graphene reconstruction takes place on a nanometer scale.

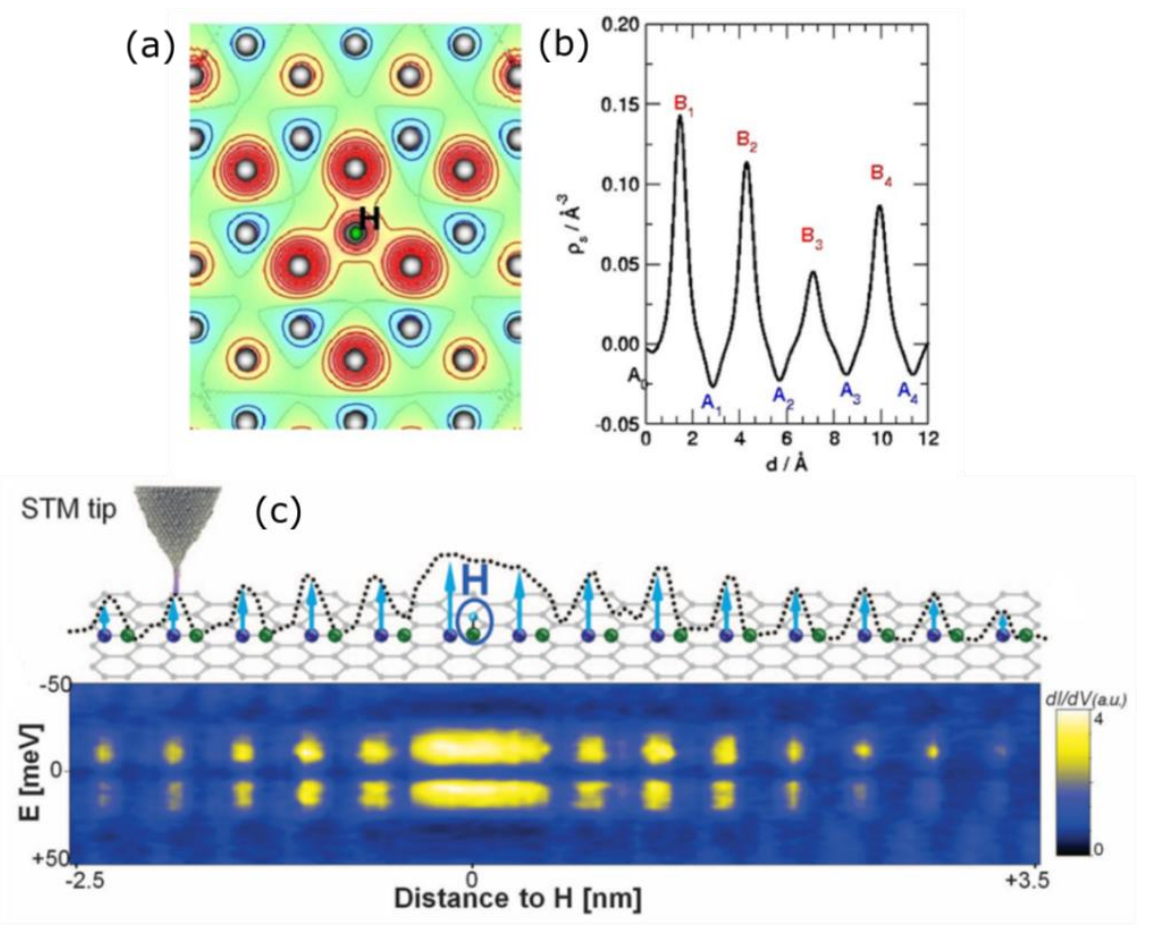

Figure 6.26: Theoretically calculated $(a, b)$ and experimentally measured $(c)$ magnetic moments induced by single $H$ atom adsorption on graphene. Panel (a): Contour map with red/blue lines for up/down magnetic moments at $0.47 \AA$ above the graphene. Panel (b): magnetic moment density on different types of $C$ atoms. " $A$ " indicates the sub-lattice, on which the $H$ atom adsorbs. " $B$ " indicates the other sub-lattice. Subscript $O$ indicates the $C$ atom, on which the $H$ atom adsorbs. Subscripts 1 4 indicate the first to fourth nearest neighboring $C$ atoms. Panel (a) and (b) reprinted from Ref. (112), with the permission of AIP Publishing. Panel (c): Experimentally determined magnetic moments as a function of distance to the adsorbed H atom via STM measurement. The conductance intensity $(d I / d V)$ indicates the magnetic moment strength. Panle (c) from Ref. (148). Reprinted with permission from AAAS. 
Hence, for hydrogen atom scatting from graphene, the motion of the hydrogen atom is not only correlated to that of the colliding $\mathrm{C}$ atom, but also simultaneously to those of its neighboring $\mathrm{C}$ atoms once it crosses the adsorption barrier. All the correlated atoms form a transient reaction complex, and the hydrogen atom transfers its translational energy to many degrees of freedom of the complex at the same time. Theoretical calculations predict that graphene sample reconstruction to the adsorption equilibrium configuration requires about $0.8 \sim 0.9 \mathrm{eV}$ (107), indicating large amount of energy transfer from the $\mathrm{H}$ atom to graphene once the transient $\mathrm{C}-\mathrm{H}$ bond is formed.

Similar large and rapid energy transfer is also observed in gas phase atom-molecule collisions. J. M. Smith et al. observed that a large amount of energy is transferred from a hot $\mathrm{H}$ atom to an acetylene $\left(\mathrm{C}_{2} \mathrm{H}_{2}\right)$ molecule during a single collision, which is labeled as super energy transfer $(149,150) . \mathrm{H}$ atom beams with energies of $2.3 \mathrm{eV}$ and $2.7 \mathrm{eV}$ are generated via molecular photolysis and then collide with room temperature $\mathrm{C}_{2} \mathrm{H}_{2}$ molecules. Two clearly separated components with small and large energy losses were observed. The experimental results have been quantitatively explained by theoretical calculations performed by Y. Han et al (151). The fast component with little energy loss is assigned to prompt scattering at large impact parameters, while the slow component with large energy loss is assigned to scattering at small impact parameters, at which a reaction complex $\mathrm{C}_{2} \mathrm{H}_{3}$ could be formed.

Figure 6.27 shows the comparison between our experimental results of $\mathrm{H}$ atoms scattering on graphene and theoretical results of $\mathrm{H}$ atoms colliding with $\mathrm{C}_{2} \mathrm{H}_{2}$ molecules. Theoretical results are used in the comparison since experiments cannot provide impact-parameter specific data. The left panel shows the energy loss distribution of $\mathrm{H}$ atom scattering on graphene at different incidence angles but the same incidence energy. The incidence energy is $1.92 \mathrm{eV}$. The incidence angle is changed from $60^{\circ}$ to $40^{\circ}$ in $2^{\circ}$ steps, corresponding to normal incidence energies of 0.48 $\mathrm{eV}$ to $1.44 \mathrm{eV}$. The energy loss is integrated over all the measured scattering angles. The right panel shows the calculated energy loss of $\mathrm{H}$ atoms colliding with $\mathrm{C}_{2} \mathrm{H}_{2}$ molecules at different impact parameters. The incidence energy of the $\mathrm{H}$ atom is $2.7 \mathrm{eV}$, and energy loss is integrated over all final outcome directions. The two energy loss distributions show striking similarities, indicating similar interaction mechanisms. Here, one should notice that the $\mathrm{x}$ axes for panel (a) and (b) are different. One is normal incidence energy, and the other one is impact parameter. At 
small normal incidence energies (large impact parameters), the $\mathrm{H}$ atom does not cross the adsorption barrier to form a transient reaction complex (too far away from the $\mathrm{C}_{2} \mathrm{H}_{2}$ molecule to form any reaction complex). The direct scattering (prompt colliding) only causes small amount of energy loss. At large normal incidence energies (small impact parameters), the $\mathrm{H}$ atom crosses the adsorption barrier and forms a transient reaction complex (form a $\mathrm{C}_{2} \mathrm{H}_{3}$ complex). The transient reaction complex formation $\left(\mathrm{C}_{2} \mathrm{H}_{3}\right.$ complex formation) causes large and rapid energy transfer to the graphene $\left(\mathrm{C}_{2} \mathrm{H}_{2}\right.$ molecule $)$. Another interesting similarity is that the energy loss of the slow component is independent of the normal incidence energy or the impact parameter. This implies that the energy losses for the two cases only depend on the magnitude of the incidence energy, once the reaction complex is formed. For scattering on graphene, this is due to the fact that the initial energies parallel and perpendicular to the surface are mixed in the complex.

AIMD calculations of $\mathrm{H}$ atom scattering on graphene predict the energy loss and scattering angle of the slow component to be independent on the interaction time (shown in Figure 6.23). Theoretical calculations on collision of $\mathrm{H}$ atoms with $\mathrm{C}_{2} \mathrm{H}_{2}$ molecules also predict similar results (shown in Figure 6 in ref (151)). But the interaction time for collision of $\mathrm{H}$ atoms with $\mathrm{C}_{2} \mathrm{H}_{2}$ molecules is on the order of several hundreds of femtoseconds, which is one order of magnitude larger than the interaction time for $\mathrm{H}$ atom scattering on graphene. This means that the energy transfer for $\mathrm{H}$ atom scattering on graphene is much more efficient than that for collision with $\mathrm{C}_{2} \mathrm{H}_{2}$ molecule. 

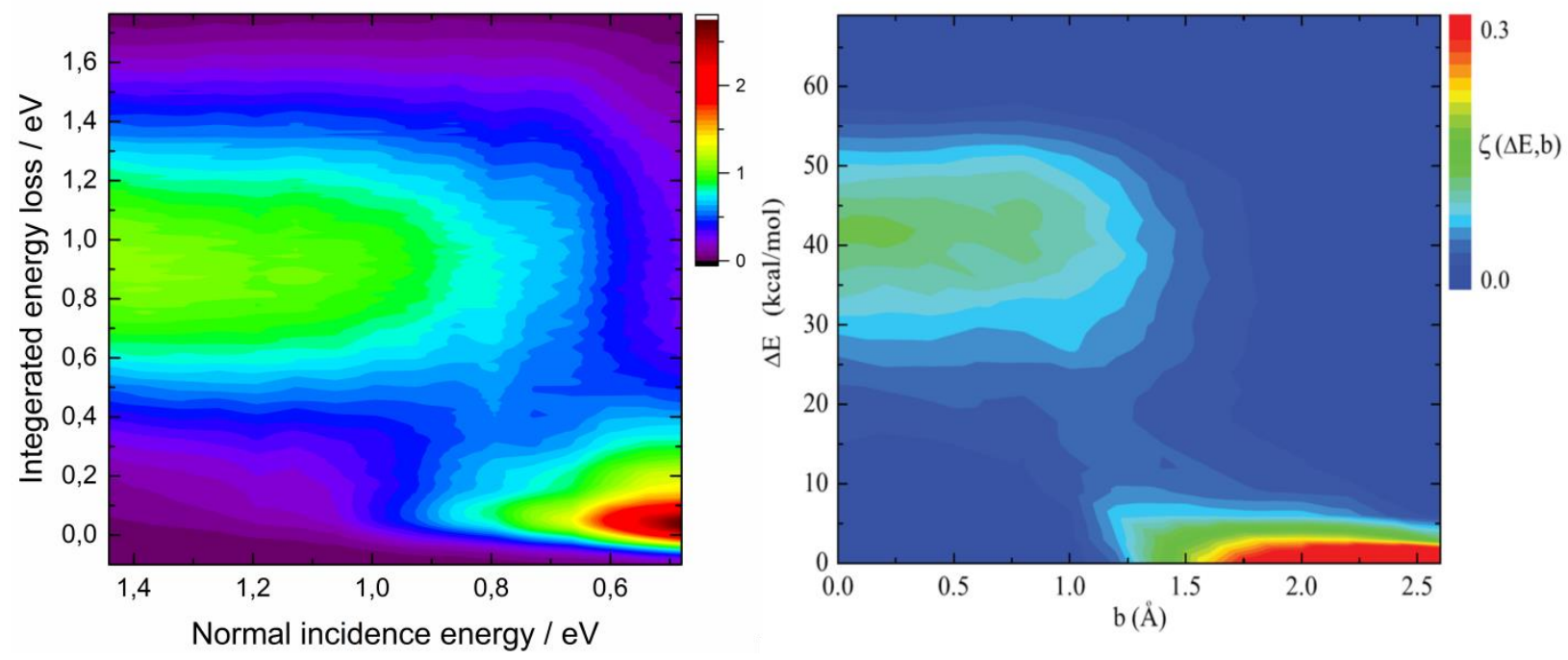

Figure 6.27: Experimentally measured energy loss distribution of $H$ atoms scattering on graphene (left panel) and theoretically calculated energy loss distribution of $H$ atoms colliding with $\mathrm{C}_{2} \mathrm{H}_{2}$ molecules (right panel). For scattering on graphene, the incidence energy is $1.92 \mathrm{eV}$, and the incidence angles are between $60^{\circ} \sim 30^{\circ}$ (normal incidence energy $0.48 \sim 1.44 \mathrm{eV}$ ). The energy loss is integrated over all measured scattering angles. For collision with $\mathrm{C}_{2} \mathrm{H}_{2}$ molecule, the incidence energy is $2.7 \mathrm{eV}$, and energy loss is integrated over all final outcome directions. Right panel reprinted from Ref. (151), with the permission of AIP Publishing.

The discussion above provides an understanding of the dynamics for the slow component from a MD point of view. Surface IVR can also be used to interpret the experimental results. Once the $\mathrm{H}$ atom crosses the adsorption barrier, it forms a highly vibrationally excited $\mathrm{C}-\mathrm{H}$ bond. The highly excited $\mathrm{C}-\mathrm{H}$ bond then redistributes its energy to the graphene phonon modes due to the anharmonic coupling between the $\mathrm{C}-\mathrm{H}$ vibrational state and the graphene phonons. Alexzander Kandratzenka et al already have some preliminary results; the detailed analysis is still on the way.

Figure 6.28 shows a preliminary result of the graphene normal mode distribution as a function of simulation time for a single trajectory, obtained from AIMD calculation of $\mathrm{H}$ atoms scattering from graphene. The $\mathrm{x}$ axis and the $\mathrm{y}$ axis are the simulation time and phonon mode label (used in the simulation). The energy of the graphene normal modes ranges from $60 \mathrm{~cm}^{-1}$ to $1583 \mathrm{~cm}^{-1}$. The number of normal modes (69 in total) is determined by the graphene slab size used in the simulation. The black line indicates $\mathrm{t}=38.9 \mathrm{fs}$, at which the $\mathrm{H}$ atom crosses the adsorption barrier. 
The blue line represents $\mathrm{t}=44 \mathrm{fs}$, at which time the minimum $\mathrm{C}-\mathrm{H}$ distance is reached. The red line indicates $\mathrm{t}=51 \mathrm{fs}$, at which time $\mathrm{H}$ atom cross the barrier again. It can be seen that a substantial number of phonon excitations occur during $\mathrm{C}-\mathrm{H}$ bond formation $(\mathrm{t}=38.9-51 \mathrm{fs})$ without any preferential phonon modes. This phonon excitation without preferential modes is common for all trajectories. The surface IVR has the same effect with simultaneously displacing several $\mathrm{C}$ atoms discussed above. Energy in the highly excited $\mathrm{C}-\mathrm{H}$ bond could flow into many phonon modes at the same time, and this causes the large and rapid energy loss.

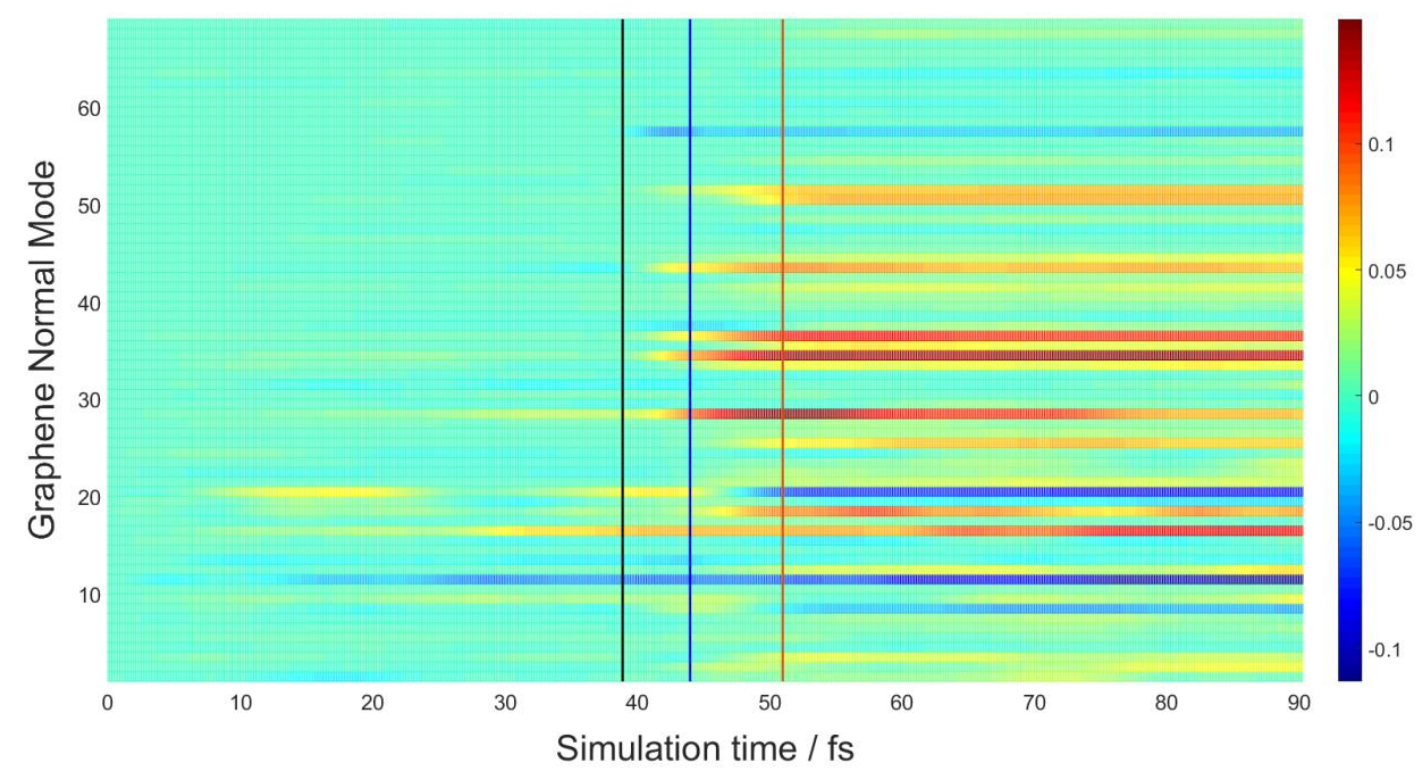

Figure 6.28: Graphene normal mode distribution as a function of simulation time for a single trajectory, obtained from AIMD calculation of $H$ atoms scattering from graphene. The incidence energy and angle are $1.92 \mathrm{eV}$ and $52^{\circ}$. Energy of the graphene normal modes ranges from 60 $\mathrm{cm}^{-1}$ to $1583 \mathrm{~cm}^{-1}$. The number of normal modes is determined by the graphene slab size used in the simulation. The black line indicates $t=38.9 f$ s (crossing the adsorption barrier). The blue line represents $t=44 f_{s}$ (minimum $C$ - $H$ distance). The red line indicates $t=51 f_{s}$ ( $H$ atom cross the barrier again) (138).

Preferential scattering angle distribution induced by dynamical steering effect: In the above discussion, I conclude that the large and rapid energy loss of the slow component is due to the formation of a transient reaction complex. This implies that the scattering angle should have a broad distribution. The independence of energy loss on the incidence angles (shown in Figure 
6.21) indicates the initial energies parallel and perpendicular to the surface are mixed during the scattering, which also suggests that a broad angular distribution should be observed. However, the experimental data show narrow scattering angular distributions with FWHMs about $30^{\circ}$, and the average scattering angles are preferentially localized around $30^{\circ}$ as shown in Figure 6.22. This preferential scattering angle distribution can be explained by dynamical steering effects that will be discussed in the following paragraph.

Dynamical steering effects have been predicted to play an important role in hydrogen atom adsorption and hydrogen molecule formation on graphene $(105,119)$. As shown in Figure 6.20, the adsorption energy of the $\mathrm{H}$ atom on graphene exhibits substantial site-dependence, indicating a highly corrugated PES. The highly corrugated PES strongly steers the $\mathrm{H}$ atom once it has crossed the adsorption barrier. Figure 6.29 (a) shows the theoretically calculated correlation between the target position and the closest $\mathrm{C}$ atom approach of the incidence $\mathrm{H}$ atom. The incidence energy and incidence angle are $1.92 \mathrm{eV}$ and $52^{\circ}$. The starting point of the arrow represents the target position, and the ending point represents the closest $\mathrm{C}$ approach (projection onto the graphene plane). All $\mathrm{H}$ atoms that are scattered back after crossing the adsorption barrier are steered by the PES to a nearby $\mathrm{C}$ atom. Figure 6.29 (b) shows the calculated correlation between the closest $\mathrm{C}$ atom approach and the final position of the scattered $\mathrm{H}$ atom (projection onto the graphene plane). The starting point of the arrow represents the closest $\mathrm{C}$ atom approach and the ending point represents the final position. The final position is $6 \AA$ above the graphene. This means that the length of the arrow is proportional to the value of the scattering angle. Zero length indicates scattering along the surface normal. The reference length in the figure indicates a scattering angle of $45^{\circ}$. It can be clearly seen that nearly all the forward scattering trajectories have scattering angles concentrated in the $10^{\circ} \sim 50^{\circ}$ range. The calculated average scattering angle is $35^{\circ}$. This preferentially super-specular scattering can be understood as follows. The $\mathrm{H}$ atoms that have crossed the barrier are first steered to a nearby $\mathrm{C}$ atom. Considering the incidence direction, those $\mathrm{H}$ atoms should then collide on the uphill side of the PES, leading to a superspecular scattering direction, similar to the case shown in Figure 6.19. Here one should notice that the top of the PES is on the honey-comb site as shown in Figure 6.20. 
From target position to closest $\mathrm{C}$ approach

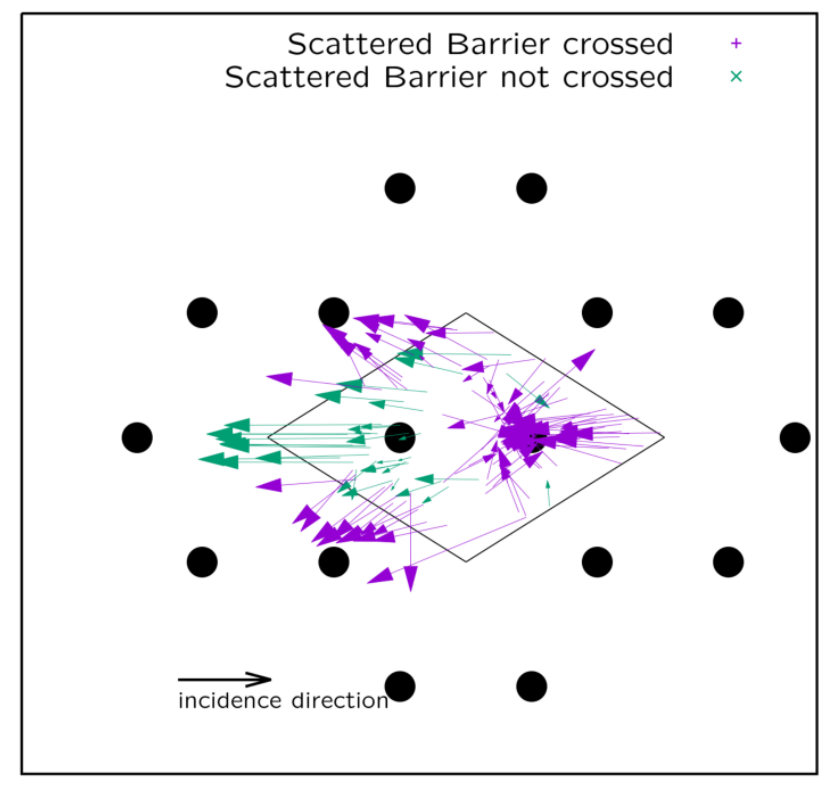

From closest $C$ approach to final position

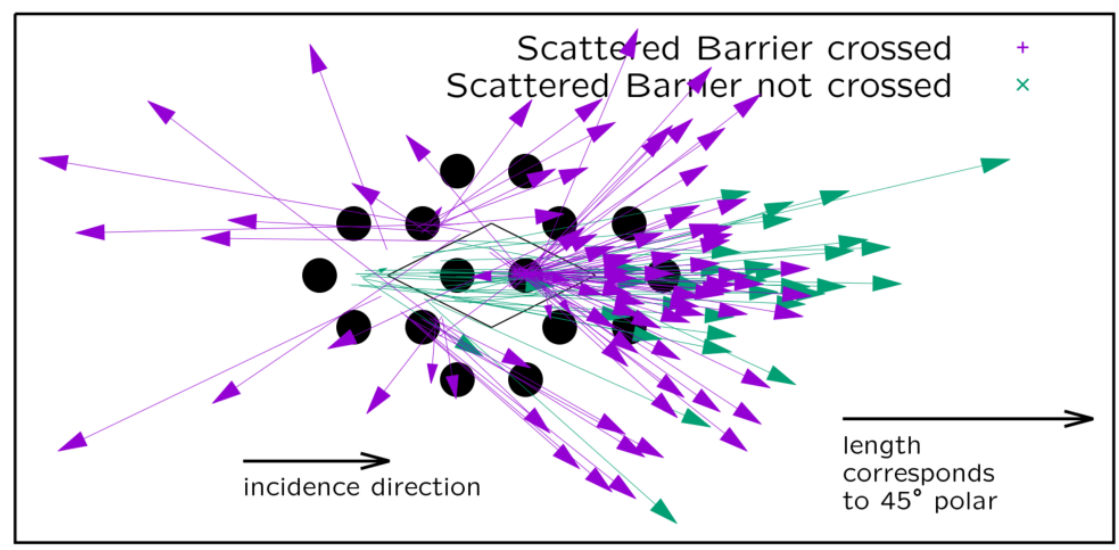

Figure 6.29: Panel (a): Theoretically calculated correlations between the target position and the closest $C$ atom approach of the incidence H atoms (the projection onto the surface). The starting point of arrow indicates the target position. The ending point indicates the closest $C$ approach. Panel (b): Theoretically calculated correlations between the closest $C$ atom approach and the final position of the scattered $H$ atoms (the projection onto the surface). The starting point of arrow indicates the closest $C$ approach. The ending point indicates the final position. The length of the arrows is proportional to the scattering angle. The incidence energy and incidence angle are $1.92 \mathrm{eV}$ and $52^{\circ}$, respectively (138). 
Dynamical steering effects also explain the disappearance of the fast component at high normal incidence energies. Theoretical calculations predict that the hydrogen atom adsorption can only take place on top sites of graphene. Potential energies above the bridge site and honey-comb center are purely repulsive. However, incidence atoms that are aimed at bridge sites and honeycomb centers can still find paths to reach the adsorption sites, due to steering by the corrugated PES. Dynamical steering effects also cause site specific scattering as shown in Figure 6.30. Incidence $\mathrm{H}$ atoms that are aimed at different sites have different fates.

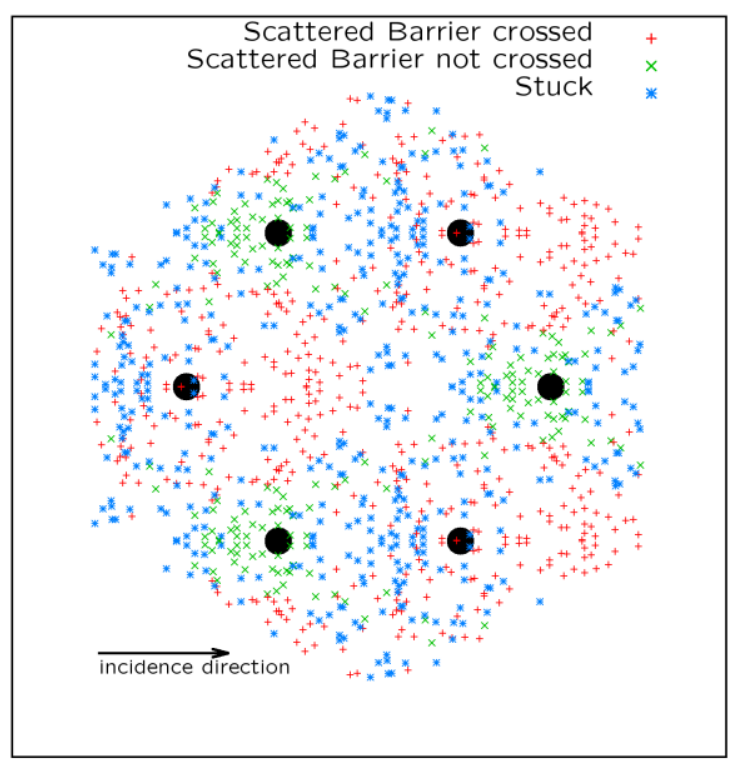

Figure 6.30: Site-specific scattering of $H$ atoms on graphene from AIMD simulation. The incidence energy and incidence angle are $1.92 \mathrm{eV}$ and $52^{\circ}$. Points in figure indicate the target position of the incidence H atoms (138). 


\subsection{Summary on H/D atoms scattering from epitaxial graphene on $\operatorname{Pt}(111)$}

In this chapter, I discussed H/D atoms scattering from epitaxial graphene on $\operatorname{Pt}(111)$ at zero surface coverage. The H/D atom adsorption on graphene is a chemically activated process. Unlike H/D atoms scattering on metal and insulator surfaces, the scattering on graphene shows a bimodal distribution. The fast component originates from atoms scattered back before crossing the adsorption barrier, while the slow component originates from atoms scattered back after crossing the adsorption barrier. The fast component exhibits small energy loss and near-specular scattering angles. In contrast, the slow component shows large and rapid energy loss. By monitoring changes in the peak-to-peak ratio of the slow to the fast component, the zero surface coverage adsorption threshold on graphene is determined in the range of $0.41-0.48 \mathrm{eV}$ for the $\mathrm{H}$ atom, and 0.43-0.47 eV for the D atom. Combined with DFT based AIMD calculations, we conclude that the fast component is quasi-elastic scattering of H/D atoms on a corrugated surface. The large and rapid energy loss of the slow component is because of the formation of a transient reaction complex. Large portion of the energy loss is due to the tug-of-war mechanism. Fast surface IVR can also be used to interpret the experimental results. The narrow angular distribution of the slow component is due to the dynamical steering effects. The observed dynamics mainly arises from the $\pi$ resonance structure of graphene and should also apply to interaction of hydrogen atoms with large aromatic molecules.

Non-adiabatic electronic excitations may have minor or no effects, even though there is no band gap between the conduction and valance band in graphene. Non-adiabatic electronic excitations could originate from two aspects. One is the non-adiabatic excitation of electrons in the $\operatorname{Pt}(111)$ substrate via dynamic dipole moments interaction. This has been calculated as negligible for $\operatorname{Pt}(111)$ (133). Another aspect concerns the non-adiabatic electronic excitations in epitaxial graphene. The weak non-adiabatic excitation in graphene is probably because of the low available electronic density of states near the Dirac point. Hydrogen atoms on graphene creates a local insulating area around the adsorption site, this could be another reason of the minor nonadiabatic excitation (112). Based on the good agreement between experimental data and AIMD results, I conclude that the energy transfer between the H/D atom and the epitaxial graphene on $\mathrm{Pt}(111)$ is mainly adiabatic at incidence energies similar to our experiments (below $3.5 \mathrm{eV}$ ). 


\section{Summary and outlook}

In this thesis, $\mathrm{H}$ and $\mathrm{D}$ atoms scattering from single crystal metal surfaces, insulating surfaces and epitaxial graphene are studied. A state-of-the-art machine was built to conduct this study. Two widely known techniques in gas phase experiments, atom beam generation via photolysis and Rydberg atom tagging time-of-flight, are combined in the surface scattering study. $\mathrm{H}$ atom beams with narrow energy distributions are generated via laser photodissociation of hydrogen halide molecules and scattered from well-defined single crystal surfaces. The time-of-flight spectrum of the scattered $\mathrm{H}$ atoms is measured using the Rydberg atom tagging method. A complete set of angular and speed distribution is measured with a rotatable detector at high angular resolution $\left(3^{\circ}\right)$. The single crystal sample is mounted on a home-designed 6-axis manipulator. The surface can be cleaned and characterized via standard techniques including ion sputtering, electron bombardment heating, low energy electron diffraction and Auger electron spectroscopy.

$\mathrm{H}$ atoms scattering from Xe layer exhibit small energy loss and narrow translational energy distribution. The dynamics can be understood within a simple binary collision model. In contrast, $\mathrm{H}$ atoms scattering from $\mathrm{Au}(111)$ shows large energy loss and broad translational energy distribution and is mainly non-adiabatic. $\mathrm{H}$ and $\mathrm{D}$ atoms scattering from $\mathrm{Au}(111)$ with the same incidence energy show a minor isotope effect. The small isotope effect is explained by the compensation between adiabatic phonon excitation proportional to the atomic mass and nonadiabatic electron-hole pair excitation proportional to the speed of the atom. The experimental findings are quantitatively reproduced by a MD simulation including non-adiabatic electron-hole pair excitations as electronic friction.

Unlike H/D atoms scattering on metal and insulator surfaces, the scattering on graphene shows a bimodal translational energy and scattering angle distribution. The fast component originates from atoms scattered back before crossing the adsorption barrier, while the slow component originates from atoms scattered back after crossing the adsorption barrier. The fast component exhibits small energy loss and near-specular scattering angles. In contrast, the slow component shows large and rapid energy loss. By monitoring changes in the peak-to-peak ratio of the slow to the fast component, the zero surface coverage adsorption threshold on graphene are determined in the range of $0.41 \sim 0.48 \mathrm{eV}$ for the $\mathrm{H}$ atom, and $0.43 \sim 0.47 \mathrm{eV}$ for the $\mathrm{D}$ atom. 
Combined with DFT based AIMD calculations, we conclude that the fast component is H/D atom quasi-elastic scattering on a corrugated surface. The large and rapid energy loss of the slow component is because of the formation of a transient reaction complex. Due to the $\pi$ resonance structure of graphene, a $\mathrm{H}$ atom colliding with one $\mathrm{C}$ atom will cause simultaneous displacements of the neighboring $\mathrm{C}$ atoms, leading to the formation of a transient reaction complex. Large portion of the energy loss is due to the tug-of-war mechanism. Fast surface IVR can also be used to interpret the experimental results. The narrow angular distribution of the slow component is due to dynamical steering effects. The observed dynamics mainly arises from the $\pi$ resonance structure of graphene and should also apply to interaction of hydrogen atoms with large aromatic molecules.

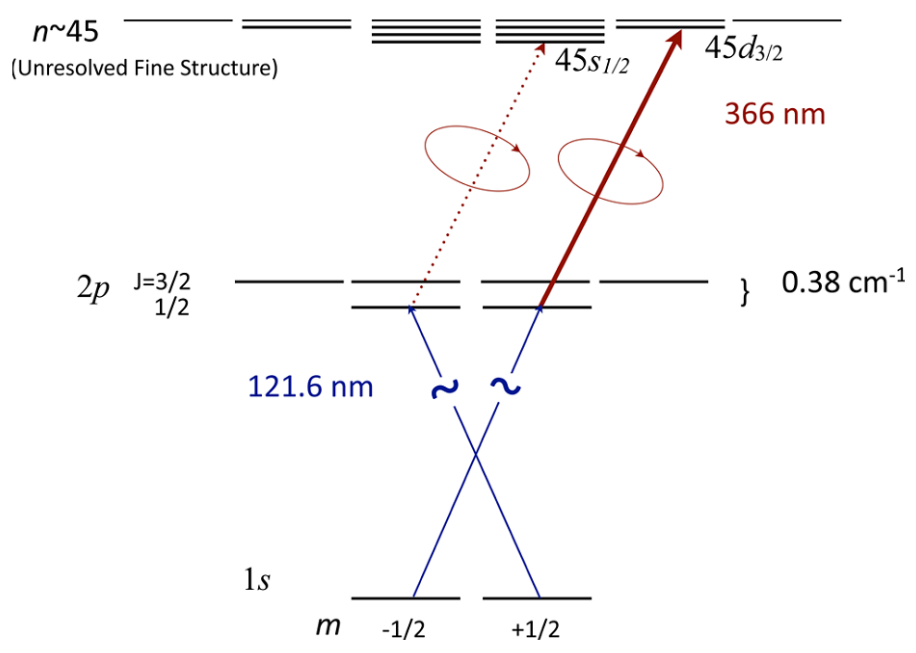

Figure 7.1: Spin-sensitive Rydberg atom tagging scheme. The linearly polarized $121.6 \mathrm{~nm}$ laser excites the two $H 1$ s magnetic sublevels to the $2 p$ level. The circularly polarized narrow bandwidth $366 \mathrm{~nm}$ laser is tuned to only excite atoms at $2 p_{1 / 2}$ level to the high Rydberg states with $n=45$. The two magnetic sublevels $m=-1 / 2$ and $m=1 / 2$ of $2 p_{1 / 2}$ level are excited to the $45 s_{1 / 2}$ and the $45 d_{3 / 2}$, respectively. The radial integrals for transitions to the $45 s_{1 / 2}$ are about 37 times smaller than those to the $45 d_{3 / 2}$. The transition to $45 s_{1 / 2}$ can be neglected, leading to spin specific detection. Reprinted with permission from Ref. (152). Copyright (2013) American Chemical Society. 
For hydrogen atoms scattering from metal surfaces, spin dynamics has drawn attention for decades. During the scattering of $\mathrm{H}$ atoms from metal surfaces, electron may jump from the metal onto the $\mathrm{H}$ atom, leading to the formation of a transient $\mathrm{H}^{-}$. When the electron jumps back from the $\mathrm{H}^{-}$, the initial spin state of the $\mathrm{H}$ atom may be changed. Spin polarized $\mathrm{H}$ atom beam has been generated by laser photolysis of hydrogen halides $(43,153,154)$. Recently, a spin-sensitive hydrogen Rydberg atom tagging method was developed (shown in Figure 7.1), allowing the spin polarization and velocity distribution to be measured at the same time (152). Combining these two methods with our surface scattering machine, spin dynamics of hydrogen atoms scattering from metal surfaces can then be studied.

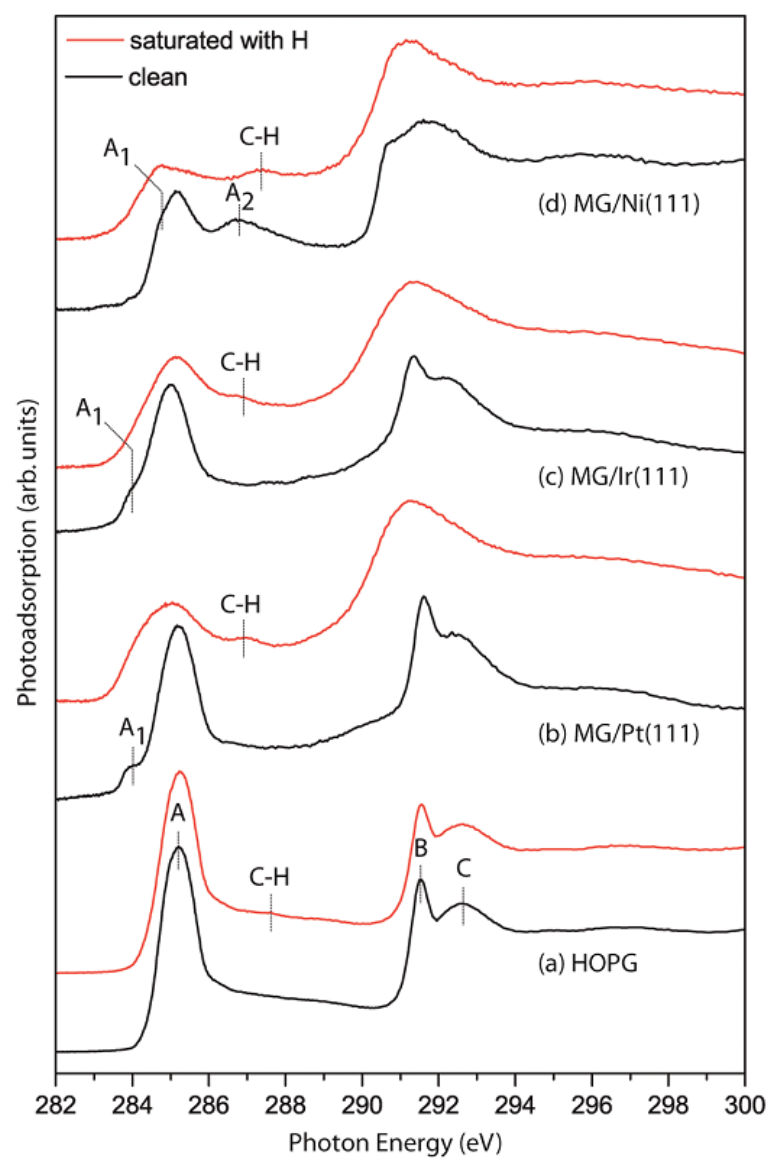

Figure 7.2: The C 1s NEXAFS spectra from the pristine (black curves) and the hydrogen saturated (red curves) samples. The angle of incidence is $50^{\circ} \mathrm{C}$ in all cases. (a) $\mathrm{HOPG}$, (b) $\mathrm{MG}$ on Pt(111), (c) MG on Ir(111), and (d) MG on Ni(111). Mark A, B and C indicates the signature of the $\pi^{*}(A), \sigma^{*}(B$ and $C)$ states in graphene. Mark $C$ - $H$ indicates the signature of $C-H$ bond. Reprinted with permission from Ref. (96). Copyright (2010) American Chemical Society. 
As discussed in Chapter 6, interaction of $\mathrm{H}$ atoms with epitaxial graphene can be influenced by the underlying substrates. Another interesting project would be the comparison of $\mathrm{H}$ atoms scattering from graphene on various substrates, such as $\mathrm{Ni}$, Ir, Ru and Pt. Hydrogen atoms scattering from highly oriented pyrolytic graphene (HOPG) is also an interesting system for comparison. Figure 7.2 shows near edge X-ray absorption fine structure spectra of $\mathrm{H}$ saturated graphene on different substrates. Clear differences are observed for different substrates. $\mathrm{H}$ atoms surface scattering measurements could provide valuable data about the interaction strength between epitaxial graphene and the underlying substrate. 


\section{References}

1. W. Heitler, F. London, Wechselwirkung neutraler Atome und homöopolare Bindung nach der Quantenmechanik. Zeitschrift für Physik 44, 455 (1927).

2. H. Eyring, M. Polanyi, Über einfache Gasreaktionen. Zeitschrift fur Physikalische Chemie B 12, 279 (1931).

3. M. Karplus, R. N. Porter, R. D. Sharma, Exchange Reactions with Activation Energy. I. Simple Barrier Potential for (H, H2). The Journal of chemical physics 43, 3259 (1965).

4. V. Griffing, J. Vanderslice, The Activation Energy of the $\mathrm{H}+\mathrm{H} 2$ Reaction. The Journal of chemical physics 22, 1142 (1954).

5. K. A. Peterson, D. E. Woon, T. H. Dunning, Benchmark calculations with correlated molecular wave functions. IV. The classical barrier height of the $\mathrm{H}+\mathrm{H} 2 \rightarrow \mathrm{H} 2+\mathrm{H}$ reaction. The Journal of chemical physics 100, 7410 (1994).

6. N. T. Goldberg et al., Vibrationally inelastic H + D2 collisions are forward-scattered. Proc Natl Acad Sci U S A 105, 18194 (Nov 25, 2008).

7. S. J. Greaves et al., Vibrational excitation through tug-of-war inelastic collisions. Nature 454, 88 (Jul 3, 2008).

8. G. Federici et al., Plasma-material interactions in current tokamaks and their implications for next step fusion reactors. Nucl Fusion 41, 1967 (Dec, 2001).

9. L. Schlapbach, A. Zuttel, Hydrogen-storage materials for mobile applications. Nature 414, 353 (Nov 15, 2001).

10. G. S. Higashi, Y. J. Chabal, G. W. Trucks, K. Raghavachari, Ideal hydrogen termination of the Si (111) surface. Applied Physics Letters 56, 656 (1990).

11. M. P. Andersson et al., Structure sensitivity of the methanation reaction: $\mathrm{H}-2$-induced $\mathrm{CO}$ dissociation on nickel surfaces. J Catal 255, 6 (Apr 1, 2008).

12. K. Greet-Jan, Frontiers in Surface Scattering Simulations. Science 321, 794 (2008).

13. M. Born, R. Oppenheimer, Quantum theory of molecules. Annalen Der Physik 84, 0457 (1927, 1927).

14. J. C. Tully, Perspective on "Zur quantentheorie der molekeln" - Born M, Oppenheimer R (1927) Ann Phys 84 : 457. Theoretical Chemistry Accounts 103, 173 (Feb, 2000).

15. A. M. Wodtke, J. C. Tully, D. J. Auerbach, Electronically non-adiabatic interactions of molecules at metal surfaces: Can we trust the Born-Oppenheimer approximation for surface chemistry? International Reviews in Physical Chemistry 23, 513 (Oct-Dec, 2004).

16. B. Gergen, H. Nienhaus, W. H. Weinberg, E. W. McFarland, Chemically induced electronic excitations at metal surfaces. Science 294, 2521 (Dec 21, 2001).

17. H. Nienhaus et al., Direct detection of electron-hole pairs generated by chemical reactions on metal surfaces. Surface Science 445, 335 (Jan 20, 2000).

18. H. Nienhaus et al., Electron-hole pair creation at $\mathrm{Ag}$ and $\mathrm{Cu}$ surfaces by adsorption of atomic hydrogen and deuterium. Physical review letters 82, 446 (Jan 11, 1999).

19. H. Nienhaus, Electronic excitations by chemical reactions on metal surfaces. Surf Sci Rep 45, 3 (2002).

20. U. Hagemann, D. Krix, H. Nienhaus, Electronic Excitations Generated by the Deposition of Mg on Mg Films. Physical review letters 104, (Jan 15, 2010).

21. D. Krix, R. Nunthel, H. Nienhaus, Generation of hot charge carriers by adsorption of hydrogen and deuterium atoms on a silver surface. Physical Review B 75, (Feb, 2007).

22. U. Bischler, P. Sandl, E. Bertel, T. Brunner, W. Brenig, Sticking, Adsorption, and Absorption of Atomic-H on Cu(110). Physical review letters 70, 3603 (Jun 7, 1993). 
23. J. R. Trail, D. M. Bird, M. Persson, S. Holloway, Electron-hole pair creation by atoms incident on a metal surface. Journal of Chemical Physics 119, 4539 (Aug 22, 2003).

24. J. K. Norskov, B. I. Lundqvist, Correlation between Sticking Probability and Adsorbate-Induced Electron-Structure. Surface Science 89, 251 (1979).

25. G. P. Brivio, T. B. Grimley, The Sticking Coefficient of Hydrogen-Atoms on a Metal - Exact Results for a Model Hamiltonian. Surface Science 161, L573 (1985).

26. K. Golibrzuch, N. Bartels, D. J. Auerbach, A. M. Wodtke, The Dynamics of Molecular Interactions and Chemical Reactions at Metal Surfaces: Testing the Foundations of Theory. Annual review of physical chemistry, (2015).

27. T. H. Johnson, Diffraction of hydrogen atoms. Physical Review 37, 847 (Apr, 1931).

28. H. U. Finzel et al., Atom-surface scattering with velocity-slected $\mathrm{H}$ and D-atomic beams from LiF and $\mathrm{NaF}(001)$. Surface Science 49, 577 (1975, 1975).

29. L. Schnieder, W. Meier, K. H. Welge, M. N. R. Ashfold, C. M. Western, Photodissociation Dynamics of H2s at $121.6 \mathrm{Nm}$ and a Determination of the Potential-Energy Function of Sh(A2Sigma+). Journal of Chemical Physics 92, 7027 (Jun 15, 1990).

30. J. E. Hurst et al., Observation of Direct Inelastic Scattering in the Presence of TrappingDesorption Scattering: Xe on Pt(111). Physical review letters 43, 1175 (1979).

31. J. E. Hurst, L. Wharton, K. C. Janda, D. J. Auerbach, Trapping-desorption scattering of argon from Pt(111). The Journal of chemical physics 83, 1376 (1985).

32. J. E. Hurst, L. Wharton, K. C. Janda, D. J. Auerbach, Direct Inelastic-Scattering Ar from Pt(111). Journal of Chemical Physics 78, 1559 (1983).

33. E. Ghio, L. Mattera, C. Salvo, F. Tommasini, U. Valbusa, Vibrational spectrum of H and D on the (0001) graphite surface from scattering experiments. The Journal of chemical physics 73, 556 (1980).

34. P. Hohenberg, W. Kohn, Inhomogeneous Electron Gas. Physical Review B 136, B864 (1964).

35. W. Kohn, L. J. Sham, Quantum Density Oscillations in an Inhomogeneous Electron Gas. Physical Review 137, 1697 (1965).

36. P. J. Knowles, J. S. Andrews, R. D. Amos, N. C. Handy, J. A. Pople, Restricted Moller-Plesset Theory for Open-Shell Molecules. Chemical Physics Letters 186, 130 (Nov 8, 1991).

37. E. F. Valeev, T. Daniel Crawford, Simple coupled-cluster singles and doubles method with perturbative inclusion of triples and explicitly correlated geminals: The CCSD(T)R12 model. The Journal of chemical physics 128, 244113 (Jun 28, 2008).

38. B. Lepetit, B. Jackson, Sticking of hydrogen on supported and suspended graphene at low temperature. Physical review letters 107, 236102 (Dec 2, 2011).

39. Y. Wang, H. J. Qian, K. Morokuma, S. Irle, Coupled cluster and density functional theory calculations of atomic hydrogen chemisorption on pyrene and coronene as model systems for graphene hydrogenation. The journal of physical chemistry. A 116, 7154 (Jul 5, 2012).

40. W. Kohn, L. J. Sham, Self-Consistent Equations Including Exchange and Correlation Effects. Physical Review 140, 1133 (1965).

41. L. J. Sham, W. Kohn, 1-Particle Properties of an Inhomogeneous Interacting Electron Gas. Physical Review 145, 561 (1966).

42. A. Gross, Reactions at surfaces studied by ab initio dynamics calculations. Surf Sci Rep 32, 291 (1998).

43. A. Brown, Photodissociation of $\mathrm{HI}$ and DI: polarization of atomic photofragments. The Journal of chemical physics 122, 84301 (Feb 22, 2005).

44. J. D. White, J. Chen, D. Matsiev, D. J. Auerbach, A. M. Wodtke, Conversion of large-amplitude vibration to electron excitation at a metal surface. Nature 433, 503 (Feb 3, 2005). 
45. Y. H. Huang, C. T. Rettner, D. J. Auerbach, A. M. Wodtke, Vibrational promotion of electron transfer. Science 290, 111 (Oct 6, 2000).

46. B. Mildner, E. Hasselbrink, D. Diesing, Electronic excitations induced by surface reactions of $\mathrm{H}$ and D on gold. Chemical Physics Letters 432, 133 (Dec 4, 2006).

47. D. Diesing, E. Hasselbrink, Chemical energy dissipation at surfaces under UHV and high pressure conditions studied using metal-insulator-metal and similar devices. Chemical Society Reviews 45, 3747 (Jul 7, 2016).

48. B. Schindler, D. Diesing, E. Hasselbrink, Electronic Excitations in the Course of the Reaction of $\mathrm{H}$ with Coinage and Noble Metal Surfaces: A Comparison. Zeitschrift Fur Physikalische ChemieInternational Journal of Research in Physical Chemistry \& Chemical Physics 227, 1381 (Nov, 2013).

49. M. S. Mizielinski, D. M. Bird, M. Persson, S. Holloway, Newns-Anderson model of chemicurrents in $\mathrm{H} / \mathrm{Cu}$ and $\mathrm{H} / \mathrm{Ag}$. Surface Science 602, 2617 (Jul, 2008).

50. N. Shenvi, S. Roy, J. C. Tully, Nonadiabatic dynamics at metal surfaces: Independent-electron surface hopping. Journal of Chemical Physics 130, (May 7, 2009).

51. N. Shenvi, S. Roy, J. C. Tully, Dynamical Steering and Electronic Excitation in NO Scattering from a Gold Surface. Science 326, 829 (Nov 6, 2009).

52. M. S. Mizielinski, D. M. Bird, M. Persson, S. Holloway, Electronic nonadiabatic effects in the adsorption of hydrogen atoms on metals. Journal of Chemical Physics 122, (Feb 22, 2005).

53. M. S. Mizielinski, D. M. Bird, M. Persson, S. Holloway, Spectrum of electronic excitations due to the adsorption of atoms on metal surfaces. Journal of Chemical Physics 126, (Jan 21, 2007).

54. S. M. Janke, D. J. Auerbach, A. M. Wodtke, A. Kandratsenka, An Accurate Full-Dimensional Potential Energy Surface for H-Au(111): Importance of Nonadiabatic Electronic Excitation in Energy Transfer and Adsorption. Journal of Chemical Physics 143, 124708 (2015).

55. A. B. Alekseyev, H. P. Liebermann, D. B. Kokh, R. J. Buenker, On the ultraviolet photofragmentation of hydrogen iodide. Journal of Chemical Physics 113, 6174 (Oct 15, 2000).

56. S. R. Langford, P. M. Regan, A. J. Orr-Ewing, M. N. R. Ashfold, On the UV photodissociation dynamics of hydrogen iodide. Chemical Physics 231, 245 (Jun 1, 1998).

57. L. Schnieder, K. Seekamprahn, F. Liedeker, H. Steuwe, K. H. Welge, Hydrogen-Exchange Reaction H + D2 in Crossed Beams. Faraday Discussions 91, 259 (1991).

58. J. P. Marangos, N. Shen, H. Ma, M. H. R. Hutchinson, J. P. Connerade, Broadly Tunable VacuumUltraviolet Radiation Source Employing Resonant Enhanced Sum Difference Frequency Mixing in Krypton. Journal of the Optical Society of America B-Optical Physics 7, 1254 (Jul, 1990).

59. E. V. Iski, A. D. Jewell, H. L. Tierney, G. Kyriakou, E. C. H. Sykes, Controllable restructuring of a metal substrate: Tuning the surface morphology of gold. Surface Science 606, 536 (Feb, 2012).

60. O. Buenermann et al., Electron-hole pair excitation determines the mechanism of hydrogen atom adsorption. Science 350, 1346 (Dec 11, 2015).

61. X. Han, T. Balgar, E. Hasselbrink, Vibrational dynamics of hydrogen on Ge surfaces. The Journal of chemical physics 130, 134701 (Apr 7, 2009).

62. P. Guyot-Sionnest, P. H. Lin, E. M. Hiller, Vibrational dynamics of the Si-H stretching modes of the $\mathrm{Si}(100) / \mathrm{H}: 2 \times 1$ surface. The Journal of chemical physics 102, 4269 (1995).

63. X. Han, K. Laß, E. Hasselbrink, Vibrational relaxation of adsorbates at semiconductor surfaces: $\mathrm{H}$ on Ge(100). Journal of Physics: Condensed Matter 20, 224008 (2008).

64. D. Hollenbach, E. E. Salpeter, Surface Recombination of Hydrogen Molecules. The Astrophysical Journal 163, 155 (1971).

65. G. Vidali, J. E. Roser, G. Manicó, V. Pirronello, A Summary of Experimental Results on Molecular Hydrogen Formation on Dust Grain Analogues. Proceedings of the International Astronomical Union 1, 355 (2006). 
66. S. E. Huber, T. Hell, M. Probst, A. Ostermann, Numerical investigation of the elastic scattering of hydrogen (isotopes) and helium at graphite (0001) surfaces at beam energies of 1 to $4 \mathrm{eV}$ using a split-step Fourier method. Theor Chem Acc 132, 1337 (2013).

67. S. Y. Zhou et al., Substrate-induced bandgap opening in epitaxial graphene. Nature materials 6 , 770 (Oct, 2007).

68. D. Haberer et al., Tunable band gap in hydrogenated quasi-free-standing graphene. Nano letters 10, 3360 (Sep 8, 2010).

69. D. C. Elias et al., Control of graphene's properties by reversible hydrogenation: evidence for graphane. Science 323, 610 (Jan 30, 2009).

70. C. Lin et al., Direct observation of ordered configurations of hydrogen adatoms on graphene. Nano letters 15, 903 (Feb 11, 2015).

71. P. R. Wallace, The Band Theory of Graphite. Physical Review 71, 622 (1947).

72. B. LANG, A LEED study of the desorption of carbon on Pt crystal surfaces. Surface Science 53, 317 (1975).

73. T. Aizawa, R. Souda, S. Otani, Y. Ishizawa, C. Oshima, Bond softening in monolayer graphite formed on transition-metal carbide surfaces. Physical Review B 42, 11469 (1990).

74. T. A. Land, T. Michely, R. J. Behm, J. C. Hemminger, G. Comsa, STM-investigation-of-single-layergraphite-structures-produced-on-Pt-111-by-hydrocarbon-decomposition. Surface Science 264, 261 (1992).

75. K. S. Novoselov et al., Electric Field Effect in Atomically thin C atom film. Science 306, 666 (2004).

76. A. H. Castro Neto, F. Guinea, N. M. R. Peres, K. S. Novoselov, A. K. Geim, The electronic properties of graphene. Reviews of Modern Physics 81, 109 (2009).

77. K. S. Novoselov et al., Two-dimensional gas of massless Dirac fermions in graphene. Nature 438, 197 (Nov 10, 2005).

78. K. S. Novoselov et al., A roadmap for graphene. Nature 490, 192 (Oct 11, 2012).

79. L. Wirtz, A. Rubio, The phonon dispersion of graphite revisited. Solid State Communications 131, 141 (2004).

80. Mingjie Liu, Vasilii I. Artyukhov, Hoonkyung Lee, Fangbo Xu, a. B. I. Yakobson, Carbyne from First Principles: Chain of C Atoms, a Nanorod or a Nanorope. Acs Nano 7, 10075 (2013).

81. X. W. Changgu Lee1, Jeffrey W. Kysar, James Hone, Science-Measurement of the Elastic Properties and Intrinsic Strength of Monolayer Graphene. Science 321, 385 (2008).

82. A. K. Geim, Graphene Status and Prospects. Science 324, 1530 (2009).

83. P. W. Sutter, J. I. Flege, E. A. Sutter, Epitaxial graphene on ruthenium. Nature materials 7, 406 (May, 2008).

84. M. Gao et al., Epitaxial growth and structural property of graphene on Pt(111). Applied Physics Letters 98, 033101 (2011).

85. J. H. Gao et al., Graphene growth on a Pt(111) substrate by surface segregation and precipitation. Nanotechnology 23, 055704 (Feb 10, 2012).

86. J. Lahiri et al., Graphene growth and stability at nickel surfaces. New Journal of Physics 13, 025001 (2011).

87. W. C. Xuesong Li, Jinho An, Seyoung Kim, Junghyo Nah, Dongxing Yang,, A. V. Richard Piner, Inhwa Jung, Emanuel Tutuc, Sanjay K. Banerjee,, Luigi Colombo, R. S. Ruoff, Large-Area Synthesis of High-Quality and Uniform Graphene Films on Copper Foils. Science 324, 1312 (2009).

88. L. Gao, J. R. Guest, N. P. Guisinger, Epitaxial graphene on Cu(111). Nano letters 10, 3512 (Sep 8, 2010).

89. P. Sutter, J. T. Sadowski, E. Sutter, Graphene on Pt(111): Growth and substrate interaction. Physical Review B 80, (2009). 
90. T. Aizawa et al., Phonon-Dispersion of Monolayer Graphite on Pt(111) and Nbc Surfaces - Bond Softening and Interface Structures. Surface Science 260, 311 (Jan, 1992).

91. K. V. Emtsev, F. Speck, T. Seyller, L. Ley, J. D. Riley, Interaction, growth, and ordering of epitaxial graphene on SiC $\{0001\}$ surfaces: A comparative photoelectron spectroscopy study. Physical Review B 77, (2008).

92. C. Riedl, C. Coletti, T. Iwasaki, A. A. Zakharov, U. Starke, Quasi-free-standing epitaxial graphene on SiC obtained by hydrogen intercalation. Physical review letters 103, 246804 (Dec 11, 2009).

93. M. Andersen, L. Hornekær, B. Hammer, Graphene on metal surfaces and its hydrogen adsorption: A meta-GGA functional study. Physical Review B 86, (2012).

94. P. A. Khomyakov et al., First-principles study of the interaction and charge transfer between graphene and metals. Physical Review B 79, (2009).

95. A. B. Preobrajenski, M. L. Ng, A. S. Vinogradov, N. Mårtensson, Controlling graphene corrugation on lattice-mismatched substrates. Physical Review B 78, (2008).

96. M. L. Ng et al., Controlling Hydrogenation of Graphene on Transition Metals. The Journal of Physical Chemistry C 114, 18559 (2010).

97. A. Politano et al., Elastic properties of a macroscopic graphene sample from phonon dispersion measurements. Carbon 50, 4903 (2012).

98. T. Aizawa et al., Phonon-Dispersion in Monolayer Graphite Formed on $\mathrm{Ni}(111)$ and $\mathrm{Ni}(001)$. Surface Science 237, 194 (Nov, 1990).

99. V. Pirronello, C. Liu, L. Y. Shen, G. Vidali, Laboratory synthesis of molecular hydrogen on surfaces of astrophysical interest. Astrophysical Journal 475, L69 (Jan 20, 1997).

100. S. Cazaux, A. G. G. M. Tielens, Molecular Hydrogen Formation in the Interstellar Medium. The Astrophysical Journal 575, L29 (2002).

101. R. Grassi, T. Low, M. Lundstrom, Scaling of the energy gap in pattern-hydrogenated graphene. Nano letters 11, 4574 (Nov 9, 2011).

102. D. Haberer et al., Electronic properties of hydrogenated quasi-free-standing graphene. physica status solidi (b) 248, 2639 (2011).

103. K. Tada et al., Ferromagnetism in hydrogenated graphene nanopore arrays. Physical review letters 107, 217203 (Nov 18, 2011).

104. L. Jeloica, V. Sidis, DFT investigation of the adsorption of atomic hydrogen on a cluster-model graphite surface. chemical Physics Letters, 1 (1999).

105. M. Bonfanti, B. Jackson, K. H. Hughes, I. Burghardt, R. Martinazzo, Quantum dynamics of hydrogen atoms on graphene. II. Sticking. The Journal of chemical physics 143, 124704 (Sep 28, 2015).

106. M. Bonfanti, B. Jackson, K. H. Hughes, I. Burghardt, R. Martinazzo, Quantum dynamics of hydrogen atoms on graphene. I. System-bath modeling. The Journal of chemical physics 143, 124703 (Sep 28, 2015).

107. X. Sha, B. Jackson, First-principles study of the structural and energetic properties of $\mathrm{H}$ atoms on a graphite () surface. Surface Science 496, 318 (2002).

108. X. Sha, B. Jackson, D. Lemoine, B. Lepetit, Quantum studies of $\mathrm{H}$ atom trapping on a graphite surface. The Journal of chemical physics 122, 14709 (Jan 1, 2005).

109. J. Kerwin, X. Sha, B. Jackson, Classical studies of H atom trapping on a graphite surface. The journal of physical chemistry. B 110, 18811 (Sep 28, 2006).

110. D. W. Boukhvalov, M. I. Katsnelson, A. I. Lichtenstein, Hydrogen on graphene: Electronic structure, total energy, structural distortions and magnetism from first-principles calculations. Physical Review B 77, (2008).

111. J. Kerwin, B. Jackson, The sticking of $H$ and $D$ atoms on a graphite (0001) surface: the effects of coverage and energy dissipation. The Journal of chemical physics 128, 084702 (Feb 28, 2008). 
112. S. Casolo, O. M. Lovvik, R. Martinazzo, G. F. Tantardini, Understanding adsorption of hydrogen atoms on graphene. The Journal of chemical physics 130, 054704 (Feb 7, 2009).

113. S. Casolo, E. Flage-Larsen, O. M. Løvvik, G. R. Darling, G. F. Tantardini, Role of the self-interaction error in studying chemisorption on graphene from first-principles. Physical Review B 81, (2010).

114. V. V. Ivanovskaya et al., Hydrogen adsorption on graphene: a first principles study. The European Physical Journal B 76, 481 (2010).

115. S. Morisset, Y. Ferro, A. Allouche, Study of the sticking of a hydrogen atom on a graphite surface using a mixed classical-quantum dynamics method. The Journal of chemical physics 133, 044508 (Jul 28, 2010).

116. S. Cazaux, S. Morisset, M. Spaans, A. Allouche, When sticking influences H2formation. Astronomy \& Astrophysics 535, A27 (2011).

117. B. Lepetit, D. Lemoine, Z. Medina, B. Jackson, Sticking and desorption of hydrogen on graphite: a comparative study of different models. The Journal of chemical physics 134, 114705 (Mar 21, 2011).

118. D. Cortes-Arriagada, S. Gutierrez-Oliva, B. Herrera, K. Soto, A. Toro-Labbe, The mechanism of chemisorption of hydrogen atom on graphene: insights from the reaction force and reaction electronic flux. The Journal of chemical physics 141, 134701 (Oct 7, 2014).

119. S. Casolo, G. F. Tantardini, R. Martinazzo, Hydrogen Recombination and Dimer Formation on Graphite from Ab Initio Molecular Dynamics Simulations. The journal of physical chemistry. A, (Mar 8, 2016).

120. E. R. M. Davidson, J. Klimes, D. Alfe, A. Michaelides, Cooperative Interplay of van der Waals Forces and Quantum Nuclear Effects on Adsorption: $\mathrm{H}$ at Graphene and at Coronene. Acs Nano 8, 9905 (Oct, 2014).

121. T. Zecho, A. Güttler, X. Sha, B. Jackson, J. r. Küppers, Adsorption of hydrogen and deuterium atoms on the (0001) graphite surface. The Journal of chemical physics 117, 8486 (2002).

122. T. Zecho et al., Abstraction of $\mathrm{D}$ chemisorbed on graphite(0001) with gaseous $\mathrm{H}$ atoms. Chemical Physics Letters 366, 188 (Nov 25, 2002).

123. E. Areou, G. Cartry, J. M. Layet, T. Angot, Hydrogen-graphite interaction: Experimental evidences of an adsorption barrier. The Journal of chemical physics 134, 014701 (Jan 7, 2011).

124. Z. Medina, B. Jackson, Quantum studies of light particle trapping, sticking, and desorption on metal and graphite surfaces. The Journal of chemical physics 128, 114704 (Mar 21, 2008).

125. L. Hornekaer et al., Clustering of chemisorbed $H(D)$ atoms on the graphite (0001) surface due to preferential sticking. Physical review letters 97, 186102 (Nov 3, 2006).

126. R. Balog et al., Atomic hydrogen adsorbate structures on graphene. Journal of the American Chemical Society 131, 8744 (Jul 1, 2009).

127. D. Haberer et al., Evidence for a new two-dimensional C4H-type polymer based on hydrogenated graphene. Advanced materials 23, 4497 (Oct 18, 2011).

128. A. Paris et al., Kinetic Isotope Effect in the Hydrogenation and Deuteration of Graphene. Advanced Functional Materials 23, 1628 (2013).

129. W. Zhao et al., Reversible hydrogenation of graphene on Ni(111)-synthesis of "graphone". Chemistry 21, 3347 (Feb 16, 2015).

130. L. Hornekaer et al., Metastable structures and recombination pathways for atomic hydrogen on the graphite (0001) surface. Physical review letters 96, 156104 (Apr 21, 2006).

131. A. Andree, M. L. Lay, T. Zecho, J. Küpper, Pair formation and clustering of $D$ on the basal plane of graphite. Chemical Physics Letters 425, 99 (2006).

132. S. Morisset, A. Allouche, Quantum dynamic of sticking of a $\mathrm{H}$ atom on a graphite surface. The Journal of chemical physics 129, 024509 (Jul 14, 2008). 
133. S. Sakong, P. Kratzer, Hydrogen vibrational modes on graphene and relaxation of the $\mathrm{C}-\mathrm{H}$ stretch excitation from first-principles calculations. The Journal of chemical physics 133, 054505 (Aug 7, 2010).

134. S. Sakong, P. Kratzer, Isotopic effect on the vibrational lifetime of the carbon-deuterium stretch excitation on graphene. The Journal of chemical physics 135, 114506 (Sep 21, 2011).

135. S. Baouche et al., High translational energy release in H2 (D2) associative desorption from H (D) chemisorbed on C(0001). The Journal of chemical physics 125, 084712 (Aug 28, 2006).

136. P. Ruffieux et al., Hydrogen adsorption onsp2-bonded carbon: Influence of the local curvature. Physical Review B 66, (2002).

137. T. P. Goumans, J. Kastner, Hydrogen-atom tunneling could contribute to $\mathrm{H} 2$ formation in space. Angewandte Chemie 49, 7350 (Sep 24, 2010).

138. A. Kandratsenka, M. Kammler, personal communication.

139. G. Kresse, J. Hafner, Ab initiomolecular dynamics for liquid metals. Physical Review B 47, 558 (1993).

140. G. Kresse, J. Hafner, Ab initiomolecular-dynamics simulation of the liquid-metal-amorphoussemiconductor transition in germanium. Physical Review B 49, 14251 (1994).

141. J. F. G. Kresse, Efficient iterative schemes for ab initio total-energy calculations using a planewave basis set. Phys Rev B 54, 11169.

142. J. F. G. Kresse, Efficiency of ab-initio total energy calculations for metals and semiconductors using a plane-wave basis set Computational Materials Science 6, 15 (1996).

143. P. E. Blöchl, Projector augmented-wave method. Physical Review B 50, 17953 (1994).

144. D. J. G. Kresse, From ultrasoft pseudopotentials to the projector augmented-wave method. Phys Rev B 59, 1758 (1999).

145. O. K. Andersen, Linear methods in band theory. Physical Review B 12, 3060 (1975).

146. S. Casolo, R. Martinazzo, M. Bonfanti, G. F. Tantardini, Quantum dynamics of the Eley-Rideal hydrogen formation reaction on graphite at typical interstellar cloud conditions. The journal of physical chemistry. A 113, 14545 (Dec 31, 2009).

147. P. G. Jambrina, J. Aldegunde, J. F. Castillo, F. J. Aoiz, V. Saez Rabanos, Vibrationally inelastic collisions of H+D2: a comparison of quantum mechanical, quasiclassical, and experimental results. The Journal of chemical physics 130, 031102 (Jan 21, 2009).

148. H. Gonzalez-Herrero et al., Atomic-scale control of graphene magnetism by using hydrogen atoms. Science 352, 437 (Apr 22, 2016).

149. J. M. Smith et al., Chemical activation through super energy transfer collisions. Journal of the American Chemical Society 136, 1682 (Feb 5, 2014).

150. J. Ma, M. J. Wilhelm, J. M. Smith, H.-L. Dai, Large cross section for super energy transfer from hyperthermal atoms to ambient molecules. Physical Review A 93, (2016).

151. Y. C. Han, A. R. Sharma, J. M. Bowman, Quasiclassical trajectory study of fast $\mathrm{H}$-atom collisions with acetylene. The Journal of chemical physics 136, 214313 (Jun 7, 2012).

152. B. M. Broderick, Y. Lee, M. B. Doyle, O. S. Vasyutinskii, A. G. Suits, Velocity Distribution of Hydrogen Atom Spin Polarization. The Journal of Physical Chemistry Letters 4, 3489 (2013).

153. T. P. Rakitzis et al., Spin-polarized hydrogen atoms from molecular photodissociation. Science 300, 1936 (Jun 20, 2003).

154. D. N. Jodoin, A. Brown, Photodissociation of $\mathrm{HI}$ and DI: testing models for electronic structure via polarization of atomic photofragments. The Journal of chemical physics 123, 054301 (Aug 1, 2005). 


\section{Acknowledgements}

First, I would like to thank Prof. Dr. Alec M. Wodtke for giving me the opportunity to work in his group and for his support and motivation over the last several years. I also thank him for all the brilliant ideas and suggestions that he provided during my $\mathrm{PhD}$ study. I would like to thank Prof. Dr. Dirk Schwarzer for all his support and for being the second member of my thesis committee.

Next, I would like to thank Dr. Oliver Bünnermann for all the help he provided throughout my whole $\mathrm{PhD}$ time. As the leader of my $\mathrm{PhD}$ project, he was always available for solving problems. I sincerely appreciate that. I would also like to thank people who worked on the RAT machine, Yvonne Dorenkamp, Hansjochen Koeckert and Nils Hertl. Their support helped me a lot. I also thank the theoreticians in the group, Dr. Alexander Kandratsenka, Marvin Kammler and Dr. Svenja Janke for their useful discussions, for comparing experimental data with computational results.

I would like to thank Prof. Dr. Xuming Yang, Dr Chunlei Xiao, and Prof. Dr. Daniel Auerbach for their help on building the RAT machine. I would like to thank Prof. Dr. Jürgen Troe for teaching me that hard working is the most important thing for a scientist. I also thank Prof. Dr. Jörg Schröder, Prof. Dr. Ricado Mata and PD Dr. Thomas Zeuch for being a member of my examination board.

I would like to say thank you to my friend-like colleagues, Tim, Dan, Pranov, Basti, Roman, Barratt, Fabian, Theo, Li, Christoph, Alexzander, Dan, Sven, Quan, Artur, Jannis, Jan, Hinrich and all the other members in the group. Thank you for the knowledge and joy you gave me

during the last five years. Special thanks to Tim and Barratt again for the help you offered during my thesis writing.

I would like to say thank you to my family and all my friends, especially my grandmother and my parents, for everything they did for me. 